University of Tennessee Health Science Center

UTHSC Digital Commons

\title{
High Resolution Mass Spectrometry (HRMS) Based Investigation of Small Molecule, Bioactive Secondary Metabolites as Probes in the Examination of Bacterial Resistance and Virulence
}

Jerrod Stephen Scarborough

University of Tennessee Health Science Center

Follow this and additional works at: https://dc.uthsc.edu/dissertations

Part of the Medicinal and Pharmaceutical Chemistry Commons

\section{Recommended Citation}

Scarborough, Jerrod Stephen, "High Resolution Mass Spectrometry (HRMS) Based Investigation of Small Molecule, Bioactive Secondary Metabolites as Probes in the Examination of Bacterial Resistance and Virulence" (2012). Theses and Dissertations (ETD). Paper 232. http://dx.doi.org/10.21007/ etd.cghs.2012.0279.

This Dissertation is brought to you for free and open access by the College of Graduate Health Sciences at UTHSC Digital Commons. It has been accepted for inclusion in Theses and Dissertations (ETD) by an authorized administrator of UTHSC Digital Commons. For more information, please contact jwelch30@uthsc.edu. 


\title{
High Resolution Mass Spectrometry (HRMS) Based Investigation of Small Molecule, Bioactive Secondary Metabolites as Probes in the Examination of Bacterial Resistance and Virulence
}

\author{
Abstract \\ The widespread availability of antimicrobial chemotherapeutics over the last half of the twentieth century \\ has offered dramatic increases in life expectancy. Unfortunately, many pathogenic agents are exhibiting \\ ever increasing resistance to many frontline antibiotics. New chemotherapeutic agents are urgently \\ needed to combat this threat; this work seeks to illustrate applications in which mass spectrometric \\ techniques may be applied to the investigation of novel, small molecule chemotherapeutics for the \\ treatment of bacterial infections. Chapter 1 contains an introduction to mass spectrometry, as well as an \\ overview of relevant chromatographic techniques. Chapter 2 introduces the bacterium $\mathrm{F}$ tularensis and $\mathrm{M}$ \\ ulcerans and details the MS based investigation of their secreted virulence factors. Chapter 3 introduces \\ a human pathogen of particular concern, $M$ tuberculosis, and contains a detailed reporting on the MS \\ based investigation of internally synthesized compounds for the treatment of latent $\mathrm{Tb}$. \\ Document Type \\ Dissertation \\ Degree Name \\ Doctor of Philosophy (PhD) \\ Program \\ Pharmaceutical Sciences \\ Research Advisor \\ Richard E. Lee, Ph.D. \\ Keywords \\ Buruli Ulcer, Mass Spectrometry, Mycolactone, Tuberculosis

\section{Subject Categories} \\ Medicinal and Pharmaceutical Chemistry | Medicine and Health Sciences | Pharmacy and Pharmaceutical \\ Sciences
}


High Resolution Mass Spectrometry (HRMS) Based Investigation of Small Molecule, Bioactive Secondary Metabolites as Probes in the Examination of Bacterial Resistance and Virulence.

\author{
A Dissertation \\ Presented for \\ The Graduate Studies Council \\ The University of Tennessee \\ Health Science Center
}

In Partial Fulfillment

Of the Requirements for the Degree

Doctor of Philosophy

From The University of Tennessee

By

Jerrod Stephen Scarborough

December 2012 
Copyright (C) 2012 by Jerrod Stephen Scarborough. All rights reserved. 


\section{DEDICATION}

I would like to dedicate this work to my dear wife Angela, and my two loving children Nolan and Jacob. 


\section{ACKNOWLEDGEMENTS}

I would first like to thank my advisor Dr. Richard E. Lee for offering me the great opportunity to improve my analytical and scientific skills as a member in his laboratory. His guidance and support have enabled me to mature not only as an analytical chemist, but to develop a deep understanding of numerous synthetic and biological techniques. I would like to express my appreciation to all of my committee members, Drs Wei Li, Sarka Beravova, Vivian Loveless and Cynthia Jeffries who have devoted a great deal of their time and effort in offering invaluable guidance and encouragement. I would like to thank Drs Pamela Small and Robin Ireland for generously providing Ft lipid samples and conducting the numerous bioassays. I greatly appreciated the help of Dr. Bernd Meibohm for his insights into pharmacokinetic techniques as well as his lab members Drs Nageshwar Budha and Dorababu Madhura and Ashit Trivedi for their microsomal analyses and in-vivo modeling. I would like to recognize Drs Sven Britton and Alvar Grönberg for their bioanalysis of mycolactone and their continuing efforts to combat Buruli ulcer. I would especially like to thank Dr. Matthew Frank for lending his expertise in LTQ-MS lipid profiling.

I would like to thank my chemistry labmates for their daily assistance and encouragement, Drs Jiuyu Liu, Samanthi Waidyarachchi, E. Jeffery North, Philip Cherian, Ying Zhao and Daniel Hoagland. I am also indebted to our biological compatriots, Drs David Bruhn and Elizabeth Griffin and Robin Lee. I would especially like to recognize Marcus Maddox for obtaining countless MICs and Dr. Rakesh for helping me edit this work. Our laboratory efforts have been made possible through the NIH grant funding of Dr. Richard E. Lee as well as through the American Lebanese Syrian Associated Charities (ALSAC). 


\begin{abstract}
The widespread availability of antimicrobial chemotherapeutics over the last half of the twentieth century has offered dramatic increases in life expectancy. Unfortunately, many pathogenic agents are exhibiting ever increasing resistance to many frontline antibiotics. New chemotherapeutic agents are urgently needed to combat this threat; this work seeks to illustrate applications in which mass spectrometric techniques may be applied to the investigation of novel, small molecule chemotherapeutics for the treatment of bacterial infections. Chapter 1 contains an introduction to mass spectrometry, as well as an overview of relevant chromatographic techniques. Chapter 2 introduces the bacterium $F$ tularensis and $M$ ulcerans and details the MS based investigation of their secreted virulence factors. Chapter 3 introduces a human pathogen of particular concern, $M$ tuberculosis, and contains a detailed reporting on the MS based investigation of internally synthesized compounds for the treatment of latent $\mathrm{Tb}$.
\end{abstract}




\section{PREFACE}

I have always had an interest in the electromechanical aspects of technology. I remember in the $5^{\text {th }}$ grade my father returned from work with a large box containing the remains of a number of derelict computers. These 8086's were obsolete even by 1991 standards but my dad simply said, "I thought you might have fun playing with these" and handed me the box with a smile. It took me a few weeks but soon enough I sat in front of the monochrome amber glow of a fully functional computer. Since that day I have had a profound interest in electronics. I have assembled numerous computers since but none have elicited such fond memories as playing Zaxxon on what entered my possession as a box bound for the dumpster.

In a freshman general chemistry class I was introduced to concept mass spectrometry (MS). MS would soon serve to combine my lifelong interest in computers and electronics to my new love of chemistry. Upon receiving my undergraduate in chemistry I continued to develop my interest in mass spectrometer entering Dr. Richard E Lee's laboratory in pursuit of a PhD in Bioanalytical Chemistry from the University of Tennessee College of Graduate Health Sciences of which this work serves as the conclusion. 


\section{TABLE OF CONTENTS}

CHAPTER 1. INTRODUCTION TO MASS SPECTROMETERY ...........................1

The Early History of Mass Spectrometry ...............................................................

Equation $1 \quad$ Lorentz Force Law .......................................................................2

Equation 2 Electric Component of Lorentz Force (Electric Sector).......................2

Equation 3 Magnetic Component of Lorentz Force (Magnetic Sector) ...................2

Equation $4 \quad$ Newton's Second Law ..................................................................2

Equation 5 Classic Motion of Charged Particles .................................................... 3

Equation $6 \quad$ Substitution of Q ..........................................................................

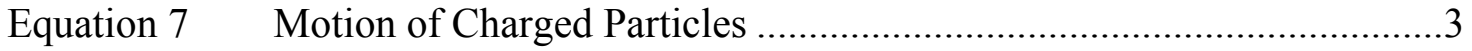

Mass Spectrometry as a Tool for Chemical Investigation ..........................................

Equation $8 \quad$ Representation of Gas Phase Electron Ionization ................................4

Equation 9 Potential Energy of an Ion in an Electric Field ..................................4

Equation 10 Kinetic Energy of an Ion in an Electric Field ....................................4

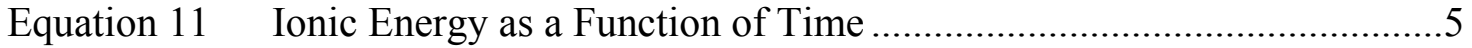

Equation 12 Ionic Energy as Expressed by Flight Time ....................................

Equation 13 Generic Time-of-Flight Equation ........................................................5

Equation 14 Approximation of Ideal Quadrupole Field Radius ............................... 7

Introduction to Chromatography .......................................................................

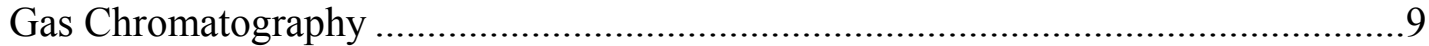

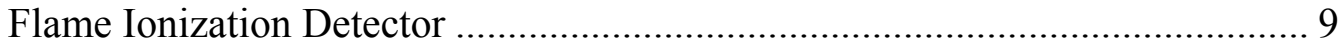

Electron Capture Detector ............................................................... 10

Liquid Chromatography ............................................................................ 10

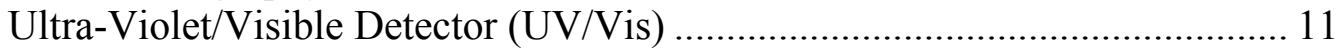

Evaporative Light Scattering Detector (ELSD) ......................................... 12

\section{CHAPTER 2. HRMS BASED INVESTGATION OF BACTERIAL \\ SECONDARY METABOLITES.................................................................................13}

An Introduction to Bacterial Secondary Metabolites ..................................................13

Decoupling of Nondestructive (NMR) and Destructive (MS) Analytical

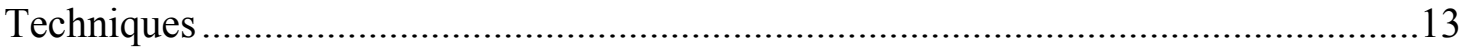

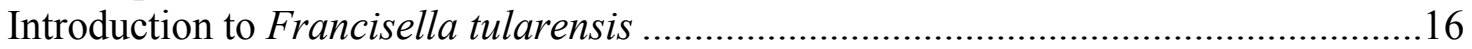

Fatty Acid Methyl Ester Derivatization and GC/MS Analysis..................................27

Ft Lipid Conclusions and Discussion .....................................................................27

Development of a Generic Bacterial Natural Product Analytical Workflow with

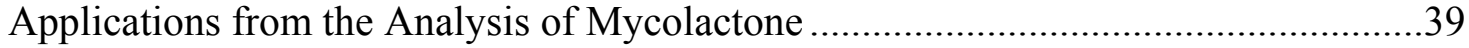

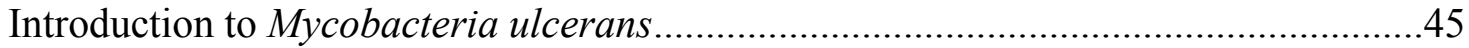

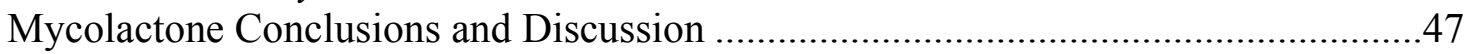

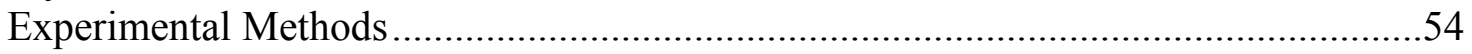

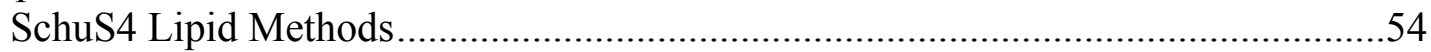

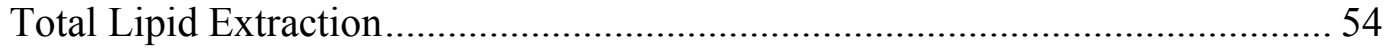

Fractionation of Cell Wall Associated Lipids (Modified Folsh) ........................ 54

Fatty Acid Methyl Esterification .................................................................. 54

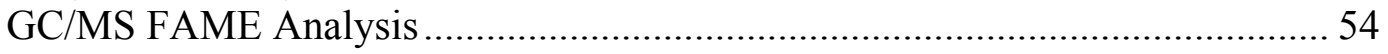




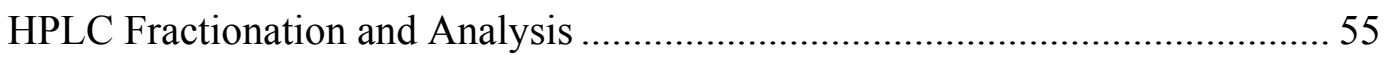

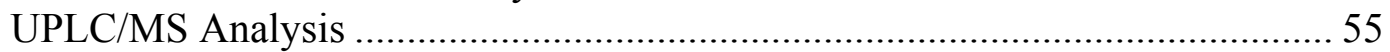

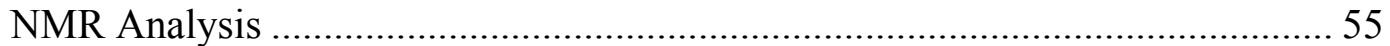

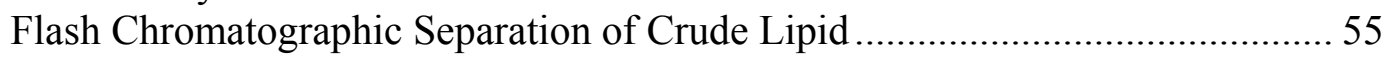

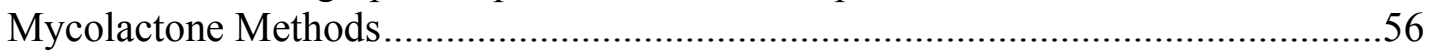

Isolation of Mycolactone from Ulcerans ........................................................ 56

Sunlight Induced Photodetoxification of Mycolactone ....................................5 56

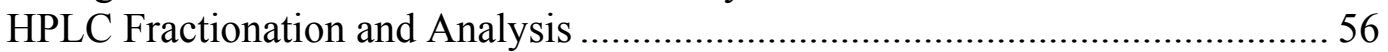

Flash Chromatographic Separation of Crude Lipid .......................................... 56

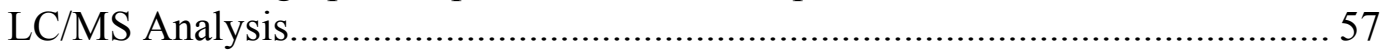

\section{CHAPTER 3. HRMS BASED METABOLIC PROFILING OF COMPOUNDS} FOR THE TREATMENT OF TUBERCULOSIS .............................................58

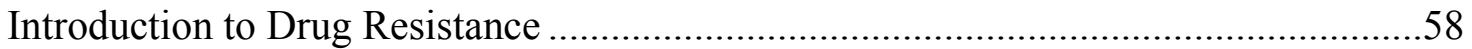

New Compounds Needed to Combat Antimicrobial Resistance ..............................58

Introduction to Antitubercular Chemotherapeutic Agents...................................59

Introduction to M. tuberculosis Pathogenicity .....................................................60

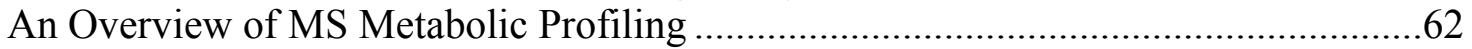

HRMS Stability Analysis of Spectinomycin Derivatives.........................................63

MassLynx and HRMS Analysis of Microsomal Stability ........................................65

MassLynx and HRMS Urinalysis of Lee Compounds ........................................65

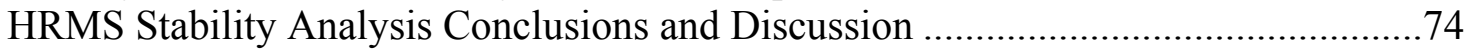

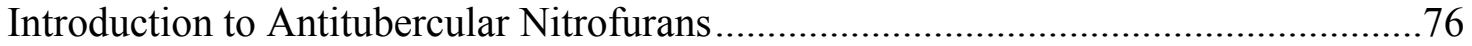

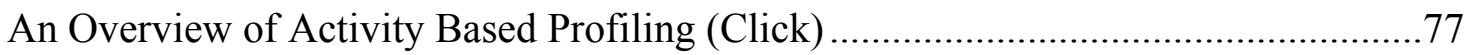

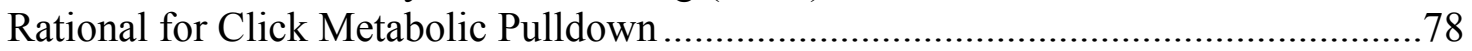

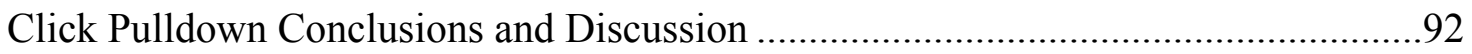

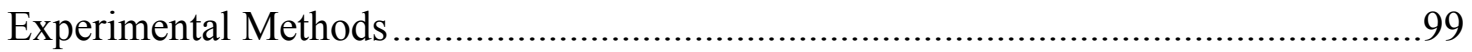

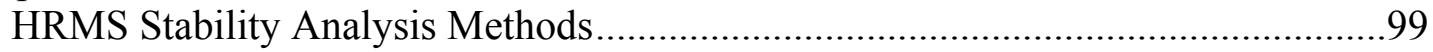

Preparation of Urinalysis Samples............................................................. 99

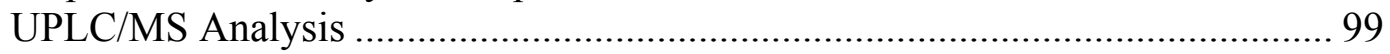

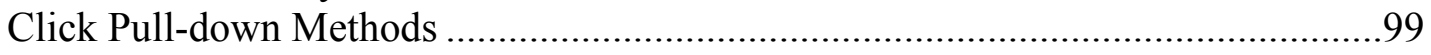

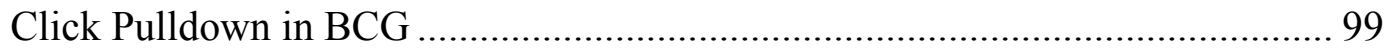

HPLC Separation of Click Conjugation Products ........................................ 100

MS Identification of Heavy Fractions......................................................... 100

LIST OF REFERENCES ......................................................................................101

APPENDIX A. MASS DIFFERENTIAL OF COMMON METABOLITES ..........119

APPENDIX B. STRUCTURES OF FAME GC/MS PEAKS \#2 - \#11 ....................121

APPENDIX C. SUPPORTING PK INFORMATION .......................................122

VITA 


\section{LIST OF TABLES}

Table 1. MICs of Acetylene Tagged Nitrofurans against Gram (+VE) Bacteria .......83

Table 2. In-vitro ADME and MIC of Click Probes ................................................95

Table 3. Common Metabolic Reactions and Their Associated Nominal Mass

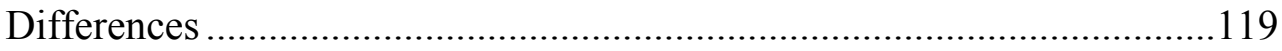

Table 4. Characteristic Fragments of Drug Conjugation via MS/MS Scanning.......120

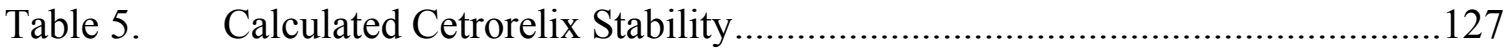




\section{LIST OF FIGURES}

Figure 1. Pictorial Representation of SchuS4 BNP Analytical Workflow

Illustrating the Extraction, Separation and Analysis of Bacterial Natural

Products

Figure 2. Detailed Workflow for the Separation and Analysis of Product Limited

Francisella tularensis Extracts

Figure 3. Activity of Modified Folch SchuS4 Partitioned Lipids with and without Addition of the Cytokine Activating LPS, with the Chloroform Fractions Predominately Responsible for Cytokine Attenuation

Figure 4. Complex Proton NMR Spectra of Crude SchuS4 Lipid Extract with

Lipid Signals Largely Masked

Figure 5. Complex 2D NMR of Crude SchuS4 Lipid Sample

Figure 6. Illustration of Typical SchuS4 Lipid 2D TLC 20

Figure 7. Bioanalysis of SchuS4 2D TLC Bands (B1-B5) at 1:100 and 1:300..... .21

Figure 8. Cytokine Deactivation Potential of Initial Flash Chromatographic

Fractions of SchuS4 Lipid Extract.

Figure 9. Cytokine Deactivation Potential of Initial Flash Chromatographic

Fractions of LVS Lipid Extract.

Figure 10. Cytokine Deactivation Potential of Initial Flash Chromatographic Fractions of SchuS4 Lipid Extract Illustrating Three Areas of Activity .....24

Figure 11. 1024 Scan Proton NMR of Pooled SchuS4 Fractions 20-24 …………........25

Figure 12. 2D HSQC of Pooled SchuS4 Fractions 20-24 Depicting the Enrichment of Fatty Acids and Phospholipids

Figure 13 Serial Concentration UPLC/MS of Non-polar LVS (Top) and SchuS4

(Bottom) Fraction Pools .28

Figure 14. Extracted Spectra from $T_{R} 4.323(463.3 \mathrm{~m} / \mathrm{z}) \Delta 44 \mathrm{~m} / \mathrm{z}$ Indicative of Polypropylene Contamination.

Figure 15. The Data Directed $\left(\mathrm{MS}^{\mathrm{e}}\right)$ TIC of SchuS4 Fractions 29-34 Displayed Similar Contamination .30

Figure 16. GC/MS Spectra of $\mathrm{HCl}-\mathrm{CH}_{3} \mathrm{OH}$ Fatty Acid Methyl Ester

Transesterification Products of LVS Acetone Soluble Lipid Extract 
Figure 17. GC/MS Spectra of $\mathrm{HCl}-\mathrm{CH}_{3} \mathrm{OH}$ Fatty Acid Methyl Ester

Transesterification Products of SchuS4 Acetone Soluble Lipid Extract .....32

Figure 18. Composite of GC/MS (HCl-MeOH) Fatty Acid Methyl Ester Transesterification Products of SchuS4 (Positive) and LVS (Negative)

Acetone Soluble Lipid Extracts

Figure 19. GC/MS Spectra of Base Catalyzed (TBAH) FAME Products of LVS

Acetone Soluble Lipid Extract

Figure 20. GC/MS Spectra of Base Catalyzed (TBAH) FAME Products of SchuS4

Acetone Soluble Lipid Extract .......................................................................35

Figure 21. Composite of Base Catalyzed (TBAH) FAME Products of LVS

(Positive) and SchuS4 (Negative) Acetone Soluble Lipid Extracts

Figure 22. GC/MS (KOH) Fatty Acid Methyl Ester Transesterification Products of SchuS4 (Positive) and LVS (Negative) Acetone Soluble Lipid Extracts Normalized for Tetradecanoate.

Figure 23. Structure of Phosphatidylethanolamine Head Group ......................................39

Figure 24. LTQ-MS of Phosphatidylethanolamine .........................................................40

Figure 25. LTQ-MS of Phosphotidylcholine …………………………………….......41

Figure 26. UPLC Targeted TQD-MS/MS of Control Mycolactone $(765.4 \mathrm{~m} / \mathrm{z})$

Served to Validate MS/MS Quantification Techniques.

Figure 27. Targeted UPLC-TQD-MS/MS of Mycolactone Subspecies $747.4 \mathrm{~m} / \mathrm{z}$ (Top) and $765.5 \mathrm{~m} / \mathrm{z}$ (Bottom) .46

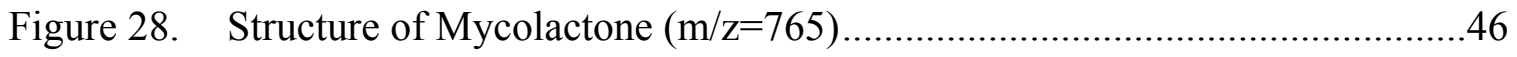

Figure 29. $765 \mathrm{~m} / \mathrm{z}$ Daughter Scan Indicating the Presence of the Mycolactone Core $(429 \mathrm{~m} / \mathrm{z})$ Prior to Sunlight Exposure (A) and Post Exposure (B)...............48

Figure 30. The Effect of Mycolactone Photoablation on Human Keratinocytes ...........49

Figure 31. The Effect of Two Mycolactone Lots on Human Keratinocytes...................50

Figure 32. Analysis of Mycolactone Stability via Differential Red (Top Set) and UV (Bottom Set) Light Flow Spectroscopy of m/z 747 (Upper) and m/z 765 (Lower) 51

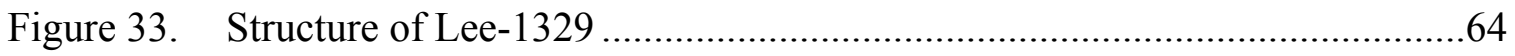

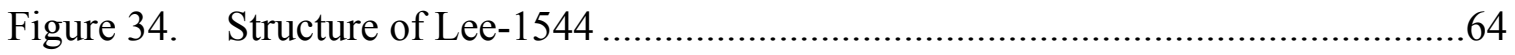




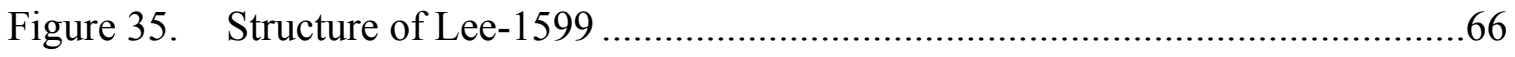

Figure 36. Possible Metabolites of Lee-1329................................................................66

Figure 37. Possible Metabolites of Lee-1544 _............................................................67

Figure 38. Preliminary Phase 2 Glucuronide Conjugation of Lee Compounds and Control in Rat Liver S9 Microsomes ............................................................68

Figure 39. Preliminary Phase 2 Sulfo-Conjugation of Lee Compounds and Control in Rat Liver S9 Microsomes

Figure 40. Preliminary Phase 2 Glutathione Conjugation of Lee Compounds and Control in Rat Liver S9 Microsomes .........................................................70

Figure 41. Extracted Ion Spectra of Lee 1544 Remains Unchanged at the Conclusion of Microsomal Incubation, Indicating 1544 Is Significantly More Stable than Initially Predicted ..............................................................71

Figure 42. Comparison of Microsomal HRMS Extracted TIC for Lee 1544.................72

Figure 43. Structure of Protonated GSH ……………................................................

Figure 44. Structure of Protonated 1544 GSH Conjugation Product...............................73

Figure 45. 1544 Urinalysis TIC and Extract Ion Spectra ..............................................75

Figure 46. Proposed Imidazole Metabolic Pathway Illustrating the Generation and Release of Highly Activated NO Species .....................................................79

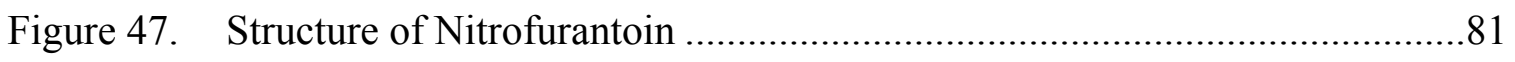

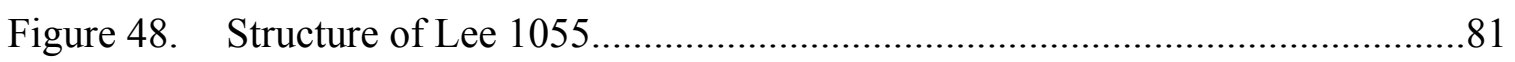

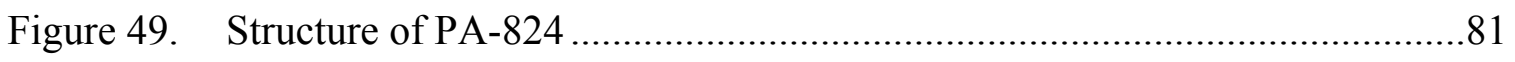

Figure 50. Generalized 1,3-Dipolar Azide-Alkyne Cycloaddition "Click" ....................82

Figure 51. Proposed Nitrofuran Metabolic Pathway Illustrating the Electron Cascade Ultimately Resulting in DNA Intercalation or Mycothiol Conjugation

Figure 52. Click Conjugation of a Light Fluorescent Probe Resulting in the Formation of the Fluor-Drug Cycloaddition Product. .85

Figure 53. Click Conjugation of a Heavy Biotinylated Probe Resulting in the Formation of the Biotin-Linker-Drug Cycloaddition Product..... . .86 
Figure 54. Fluorescence Spectra of Semi-Analytical HPLC Separation of Click Products

Figure 55. Analytical HPLC Separation of 1595 Conjugation Products with $\mathrm{MeOH}$ Organic Mobile Phase ………………………………………………….....8

Figure 56. Analytical HPLC Separation of 1595 Conjugation Products with ACN Organic Mobile Phase

Figure 57. Analytical HPLC Analytical Fluorescence Spectra of 1521 Fluorometric Conjugation Product in PBS + Catalytic TBTA . . .90

Figure 58. Analytical HPLC Fluorescence Spectra of 1521 following BCG Incubation and Click Tagging.....

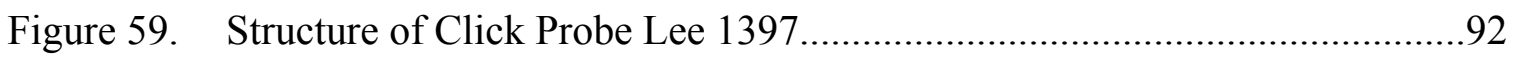

Figure 60. Structure of Click Probe Lee 1521..........................................................93

Figure 61. Structure of Click Probe Lee 1534...............................................................93

Figure 62. Structure of Click Probe Lee 1575.............................................................93

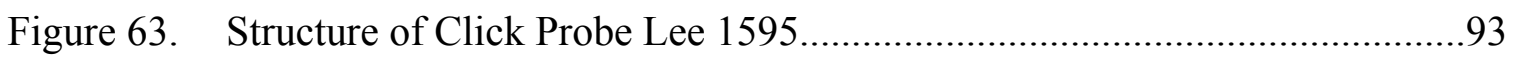

Figure 64. Structure of Click Probe Lee 1618..............................................................94

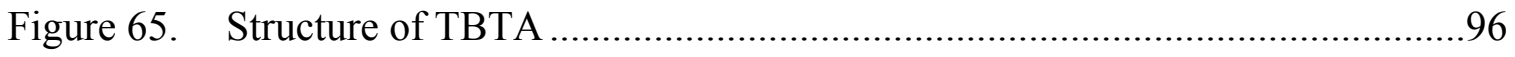

Figure 66. Structure of Proposed Mycolactone Click Probe to Enable HRMS Analysis of Polyketide Core.........................................................................98

Figure 67. Structure of Proposed Mycolactone Click Probe to Enable HRMS Analysis of Acyl Side Chain . .98

Figure 68. Structure of Proposed Linezolid Click Probe ……………...........................98

Figure 69. HRMS Identification of Nitazol Metabolite ...............................................122

Figure 70. HRMS Idenification of PD30 Metabolite .................................................123

Figure 71 Quantification of Nitazol Metabolite ........................................................124

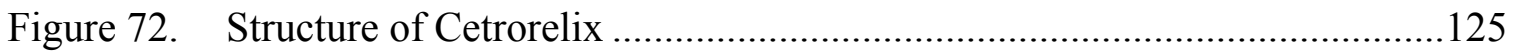

Figure 73. HRMS Stability Study of Cetrorelix ........................................................126 


\section{LIST OF ABBREVIATIONS}

ABPP

$\mathrm{AC}$

ADME

ADR

APCI

APPI

ATP

BCG

BNP

BSL

$\mathrm{ACN}$

$\mathrm{CHCl} 3$

CI

CINC

COSY

CYP

DC

DMPK

DNA

ECD

EI

ELSD

ESI

FAB

FAME

FDA

FID

FP

$\mathrm{Ft}$

GC

$\mathrm{GSH}$

GTP

HMBC

HPLC

HRMS

HSQC

ICAM

IP10
Activity Based Protein Profiling

Alternating Current

Adsorption, Distribution, Metabolism \& Excretion

Adverse Drug Reaction

Atmospheric Pressure Chemical Ionization

Atmospheric Pressure Photo Ionization

Adenine Triphosphate

Bacillus Calmette-Guérin

Bacterial Natural Product

Biological Safety Level

Acetonitrile

Chloroform

Chemical Ionization

Cytokine Induced Neutrophil Chemoattractant

Correlational Spectroscopy

Cytochrome P450

Direct Current

Drug Metabolism and Pharmacokinetics

Deoxyribonucleic Acid

Electron Capture Detector

Electron Ionization

Evaporative Light Scattering Detector

Electrospray Ionization

Fast Atom Bombardment

Fatty Acid Methyl Ester

United States Food and Drug Administration

Flame Ionization Detector

Fluorescence Polarization

Francisella tularensis

Gas Chromatography

Glutathione

Guanosine-5'-triphosphate

Heteronuclear Multiple Bond Correlation

High Performance Liquid Chromatography

High Resolution Mass Spectrometry

Heteronuclear Single Quantum Coherence

Intracellular Adhesion Molecules

Cytokine 


\begin{tabular}{ll} 
LC & Liquid Chromatography \\
LVS & Live Vaccine Strain \\
m/z & Mass/Charge Ratio \\
MALDI & Matrix Assisted Laser Desorption Ionization \\
MBC & Minimum Bactericidal Concentration \\
MCP1 & Chemokine \\
MDR & Multidrug Resistant \\
MeOH & Methanol \\
MIC & Minimum Inhibitory Concentration \\
MIP1 & Chemokine \\
MOA & Method of Action \\
MRM & Multiple Reaction Monitoring \\
MS & Mass Spectrometry \\
Mtb & Mycobacterium tuberculosis \\
Mu & Mycobacterium ulcerans \\
NADPH & Nicotinamide Adenine Dinucleotide Phosphate \\
NCE & New Chemical Entity \\
NIH & National Institutes of Health \\
NL & Neutral Loss \\
NMR & Nuclear Magnetic Resonance \\
NO & Nitrous Oxide \\
NOESY & Nuclear Overhauser Effect Spectroscopy \\
NP & Natural Product \\
PK & Pharmacokinetic \\
PL & Precursor Loss \\
PSA & Polar Surface Area \\
Q1, Q2, Q3 & Quadrupole (Location in Linear Array) \\
QIT & Quadrupole Ion Trap (Paul Trap) \\
QMA & Quadrupole Mass Analyzer \\
qTOF & Quadrupole Gated Time-of-Flight \\
RCS & Reactive Chemical Species \\
RNA & Ribonucleic Acid \\
RNS & Reactive Nitrogen Species \\
RP & Reverse Phase \\
SAR & Structure Activity Relationship \\
SDQ & Single Quadrupole Detector \\
SDS & Sodium Dodecyl Sulfate \\
SP & Standard (Normal) Phase \\
SRM & Single Reaction Monitoring \\
TBAH & Tetrabutylammonium Hydroxide \\
THF & Tetrahydrofuran \\
& \\
\hline
\end{tabular}


TNF-a

TOCSY

TOF

TQD

UPLC

$\mathrm{UV} / \mathrm{Vis}$

XDR
Tumor Necrosis Factor

Total Correlation SpectroscopY

Time-of-Flight

Triple Quadrupole Detector

Ultra Performance Liquid Chromatography

Ultraviolet / Visible Spectroscopy

Extremely Drug Resistant 


\section{CHAPTER 1. INTRODUCTION TO MASS SPECTROMETERY}

\section{The Early History of Mass Spectrometry}

In most scientific programs mass spectrometry (MS) falls largely under the guise of analytical chemistry. ${ }^{1}$ However the early history of MS is predominately the story of physics, ${ }^{2}$ more precisely the story of the electron. ${ }^{3}$ By the late 1860 's physicists had become increasingly interested in a strange phenomenon by which fluorescence could be observed around the cathode of electrochemical reaction vessels. ${ }^{4}$ While many in the physics community were investigating the nature of cathode rays, there still existed great uncertainty as to their physical composition. ${ }^{5}$

Early in his career, J.J Thomson, worked to investigate the laws which governed the transmission of electricity through gas and became interested in the propagation and composition of cathode rays. ${ }^{6}$ Convinced the observed fluorescence must be attributable to the interaction of an invisible force with the glass container, ${ }^{7}$ Thomson set out to design an apparatus capable of measuring this unknown force. ${ }^{8}$ This instrument, the first mass spectrometer, was used to measure the mass-to-charge ratio $(\mathrm{m} / \mathrm{z})$ of hydrogen gas as early as $1899 .{ }^{9}$

Thomson continued to improve his spectrometer leading him to the discovery of the electron, the elementary particle responsible for the propagation of cathode rays, for which he would receive the Nobel Prize in Physics in $1906 .{ }^{10}$ Thomson, assisted by Francis Aston, continued to study the ability of magnetic and electric fields to influence the propagation of positive ions formed by electric discharge. ${ }^{11}$ This work led to the generation of the first molecular mass spectra by positive ray spectrograph. ${ }^{12}$ While Aston and his colleagues used the new instruments to investigate chemical entities, they were primarily concerned with developing MS as to tool to tease out the physical composition of the atom. ${ }^{13}$

While the early years of MS were concerned with answering larger, theoretical questions, the dawn of World War II united many early MS pioneers together under one banner. ${ }^{2,14}$ Could MS prove to be a viable method by which to isolate the gaseous isotopes of uranium? In 1939, Albert Nier effectively answered this question by accumulating nanogram quantities of ${ }^{252} \mathrm{Pu}$ using a magnetic sector MS of his own design located at the University of Minnesota. ${ }^{15}$ Using this data, calutrons were constructed at what is today Oak Ridge National Laboratories, to perform the bomb scale separation of ${ }^{235} \mathrm{U}$ from its parent ${ }^{238} \mathrm{U}$.

Magnetic sectors are based upon the realization that electric and magnetic fields may be used to influence the trajectory of a charged gaseous (ionic) species in a sufficient vacuum as to avoid kinetic interactions (collisions). ${ }^{16}$ While Thompson's MS employed high voltages to manipulate ions, the decoupling of the electrical and magnetic components proved instrumental in the design of practical spectrometers. ${ }^{17}$ While the physics of MS remains unchanged from Thompson's first prototypes (the lighter the 
molecule, the greater the deflection) sectors were the first to influence ion trajectory based upon the duality inherent to the Lorentz Force Law. ${ }^{18}$

\section{Equation 1 Lorentz Force Law

$$
F=Q(E+v \times B)
$$

By definition; a Lorentz force is point charge due to the perturbation of an electromagnetic field.(Whereas F is the force applied to the ion, Q is the charge expressed by a particle, E denotes the contribution of an electric field, B the contribution of the magnetic field and $\mathrm{v}$ represents velocity)

\section{Equation 2 Electric Component of Lorentz Force (Electric Sector)}

$$
F=q E
$$

One of Thompson's greatest contributions to the advancement of magnetic sector instruments was his realization electric and magnetic fields may be used to differentially influence the trajectory of an ion (the principal concept on which the QMA was later built). (F again represents force, lower case $\mathrm{q}$ represents a component of the particle charge $\mathrm{Q}$, while E denotes the electric field.)

\section{Equation 3 Magnetic Component of Lorentz Force (Magnetic Sector)}

$$
F=q v B
$$

$\mathrm{F}$ again represents force, lower case $\mathrm{q}$ represents a component of the particle charge $\mathrm{Q}$, while $\mathrm{v}$ denotes velocity and $\mathrm{B}$ the magnetic field.

\section{Equation 4 Newton's Second Law}

$$
F=m \frac{d v}{d t}=m a
$$

As even the most rapidly traveling ions in a mass spectrometer fail to even encroach upon relativistic velocities, the second law may be expressed quite simply. (Whereas $\mathrm{F}$ is again representative of applied force, $\mathrm{m}$ denotes the mass of the ion and represents acceleration.) 


\section{Equation 5 Classic Motion of Charged Particles}

$$
(m / Q) a=E+v \times B
$$

The substitution of Equation 1 functionalized for net charge.

$$
\text { Equation } 6 \begin{array}{r}
\quad \text { Substitution of } \mathbf{Q} \\
z=Q / e
\end{array}
$$

As written. this equation technically equates mass number to the number of elementary charges (e) in the case of mass spectrometry; the localization of charge is often considered to be inconsequential, allowing for the direct substitution of the formally unconstrained charge number $(\mathrm{z})$.

\section{Equation 7 Motion of Charged Particles}

$$
(m / z) a=E+v \times B
$$

By war's end, multiple discrete sectors had been assembled into hyphenated spectrometers. ${ }^{19}$ Composite sector MS proved a significant advance in resolution as ions of interest could be preselected in the first field, ${ }^{20}$ then further resolved using subsequent sectors. In this way composite sectors were able to offer the performance of more modern spectrometers before the technology existed to operate quadrupoles. ${ }^{21}$

\section{Mass Spectrometry as a Tool for Chemical Investigation}

The mid 1940's saw the first commercialization of magnetic sector based instruments. ${ }^{22}$ These early commercial sectors differed from the pioneering designs of Neir in their use of electron ionization. ${ }^{23}$ While previous MS relied predominately upon an arc discharge for the generation of ions, commercial instruments relied upon the more robust electron ionization sources. ${ }^{24}$ Electron ionization (EI) mass spectra were found to be informatically superior to those of arc discharge (AD) ${ }^{25}$ The highly uniform nature of EI allowed for the formation of the first MS spectral libraries. ${ }^{26}$ 


\section{Equation 8 Representation of Gas Phase Electron Ionization}

$$
M+e^{-} \rightarrow M^{+\bullet}+2 e^{-}
$$

In this representation, $\mathrm{M}$ denotes the molecular analyte, $\mathrm{e}^{-}$the accelerated electron and $\mathrm{M}^{+}$the newly ionized analyte.

While advances in EI gaseous ion spectrometry continued throughout the late 1940 's, the incompatibility of MS with liquid analyses limited the widespread adoption of the technique. ${ }^{27}$ In many ways the advancement of MS stalled, as other analytical techniques were considered less troublesome. ${ }^{28}$ It required the commercial introduction of gas chromatography in the early 1950's to renew interest in MS as a chromatographic detector ${ }^{29}$. Though initially problematic, the interface of gas chromatography and mass spectrometry proved to be ideal. ${ }^{30} \mathrm{~A}$ sample could be prepared in a volatile solvent and injected into the GC, which desolvated the analyte and delivered it to the MS in a gaseous form. While GC had limitations (sample volatility), EI-GC/MS served to renew interest in MS development. ${ }^{31}$ As GC continued to develop, the need for faster, lower cost spectrometers became apparent. ${ }^{32}$

\section{Equation 9 Potential Energy of an Ion in an Electric Field}

$$
E_{P}=q U
$$

In this equation $\mathrm{E}_{\mathrm{p}}$ represents potential energy, $\mathrm{q}$ the fractional charge of the particle and $U$ denotes voltage (difference in potential between anode and cathode)

\section{Equation $10 \quad$ Kinetic Energy of an Ion in an Electric Field}

$$
E_{k}=\frac{1}{2} m v^{2}
$$

From Equation 9, if a charged particle at rest encounters voltage (U)(as is the case in a time-of-flight mass spectrometer) the potential energy of the particle will be completely converted to kinetic energy (Equation 10) Whereas $E_{k}$ represents the kinetic energy of an ion, $\mathrm{m}$ denotes mass and $\mathrm{v}$ represents velocity. 


\section{Equation 11 Ionic Energy as a Function of Time}

$$
E_{p}=E_{k}=\frac{1}{2} m\left(\frac{d}{t}\right)^{2}=q U
$$

In the TOF-MS the potential energy of an ion is converted to kinetic energy making Ep and Ek effectively equal. In a TOF the ion will experience an intense moment of acceleration, and then its resultant velocity will remain unchanged for the remainder of its flight down the RF free section of the flight tube. In this way the velocity of a ion as it travels through the flight tube may be determined as the path length (distance, $d$ ) and the time of flight, $t$, may be easily measured.

\section{Equation 12 Ionic Energy as Expressed by Flight Time}

$$
t=\frac{d}{\sqrt{2 U}} \sqrt{\frac{m}{q}}=k \sqrt{\frac{m}{q}}
$$

Essentially a rearrangement of Equation 11 expressed as an extrinsic function of flight time. As distance (d) and potential (U) are functions of instrument design they may be considered as constants and directly substituted for $(\mathrm{k})$ as it denotes proportionality.

\section{Equation 13 Generic Time-of-Flight Equation}

$$
t=k \sqrt{\frac{m}{z}}
$$

As in previous examples the empiric charge state (q) may be directly subsisted for the less constrained $\mathrm{z}$, yielding this familiar expression.

Throughout the early 1950's the speed of single scanning sectors greatly limited their ability to interface with $\mathrm{GC},{ }^{33}$ while the expense associated with most composite sectors remained prohibitively high. ${ }^{34}$ A number of the new time-of-flight (TOF) spectrometers were constructed and reported by $1956 .{ }^{35}$ The goal of the early TOF designers was to create an instrument with the performance characteristics of a composite sector but with a higher scanning speed and at a lower cost. ${ }^{36}$ The theory governing the operation of a TOF is quite similar to that of a sector, though it relies on time not force to afford separation. ${ }^{37}$ The key to the TOF's speed and cost savings was its amazing simplicity. Early TOF mass analyzers consisted of only a small accelerator, a length of evacuated tubing terminating in a faraday cup, itself connected to an oscilloscope. ${ }^{38}$

In a TOF mass spectrometer all incoming ions are accelerated by a known electric field, converting the ions potential energy to kinetic energy. ${ }^{39}$ These energized ions fly into a field-free drift tube under high vacuum where separation occurs based upon a particles velocity. In this way a particles velocity may be very accurately measured based 
upon the time required to complete the "flight" to the detector. ${ }^{40}$ The function of a TOF is in many ways analogous to a very accurate clock. The TOFs robustness, GC compatibility ${ }^{41}$ and relatively low operating cost (sectors consume a great deal of electricity) coupled with the development of a compatible fast atom bombardment ionization source ${ }^{42}$ secured the TOF-MS tenure in many analytical laboratories.

During the mid-1970's and early 1980's a number significant advances served to again rejuvenate MS as GC had done 2 decades prior: the commercialization and widespread adoption of reverse-phase high pressure liquid chromatography ${ }^{43}$ (HPLC), the development of electrospray ionization (ESI) ${ }^{44}$ by John Fenn and the widespread availability of computerized electronics facilitating the miniaturization of quadrupoles. ${ }^{45}$ As with GC before it, the coupling of LC to MS initially proved difficult, as the high vacuums necessary for MS were physically incompatible with the LC's liquid elutant. In 1989 John Fenn described the construction of an ESI source for the "mass spectrometry of large biomolecules." 46

Analytes dissolved into a volatile organic solvent were introduced by a low pressure pump to Fenn's ESI source. ${ }^{47}$ The analyte was then dispersed into a fine spray upon exit of the electrified capillary. The "electrospray" would then be subjected to continued desolvation. As the size of the droplets continues to decrease the density of their electric field intensifies. ${ }^{48}$ Like charges began to repel one another, greatly increasing the surface tension of the particle. At some point (Rayleigh limit), the particle can no longer tolerate this increase in tension and dissociates in smaller droplets of discrete positive and negative charge. This cycle of evaporation, charge increase, repulsion and (Coulombic) explosion continues until only carrier-free molecular ions remain. ${ }^{49}$

ESI provided a means to rapidly desolvate the LC elutant, and avoided damage to the vacuum system of the MS. While GC/MS precluded the investigation of most nonvolatile analytes, many common pharmaceutical and industrial materials were found to be compatible with LC/ESI-MS based analysis. ${ }^{50}$ Though the Fenn source was constructed for the investigation of macromolecules, lessons gleaned through its design and operation were quickly applied on a commercial scale to the investigation of large and small molecules alike. ${ }^{51}$

The early 1980's also witnessed the commercialization of spectrometers based upon Wolfgang Paul's Quadrupole Ion Trap ${ }^{52}$ (QIT) as well as the widespread availability of MS systems based upon the relatively low cost quadrupole mass analyzer ${ }^{53}$ (QMA). While QMA based instruments are generally referred to as mass spectrometers, their function is more analogous to that of a mass filter than of a conventional MS. ${ }^{54}$ In the QMA, ions within a relatively narrow mass window $(+/-1$ $\mathrm{amu}$ ) are allowed to pass through the quadrupole while ions outside the window are effectively filtered out. In this way a QMA emulates the function of a true mass spectrometer by rapidly sweeping its' small bandpass region across a broader scale. ${ }^{55}$ 
In addition to its relatively low cost, QMAs offer a number of advantages over traditional sectors and TOFs in applications where high mass accuracy is unnecessary. The small size of the QMA allows for the ion source and detector to remain in close proximity (as little as $12 \mathrm{~cm}$ ) while the strong "saddle field" effects allow the analyzer to operate at vacuum levels more easily achievable in the common laboratory ${ }^{56}\left(3 \times 10^{-5}\right.$ Torr). The typical QMA consist of four circular electrodes positioned in a radial array, at a distance proportional to their hyperbolic cross section (Equation 14). The QMA is then utilized through the application of alternating time dependant direct current (DC) and time-independent alternating current (AC) electric potentials. ${ }^{57}$

\section{Equation 14 Approximation of Ideal Quadrupole Field Radius}

$$
r=1.148 r_{0}
$$

While no man-made hyperbolic field may be considered ideal, over forty-five years of instrument design have suggested Equation 14 as the most appropriate geometry for the construction of a quadrupole hyperbolic array. Whereas $\mathbf{r}$ represents the radius of the electrodes and $\mathbf{r}_{\mathbf{0}}$ the effective quadrupole field radius

The rapidly alternating electric potentials localized around the hyperbolic electrodes serve to influence the flight of ionic species introduced into the QMA.* While the electrical gradients refresh at a very high frequency $(>2 \mathrm{kHz})$, an effort can be made to visualize a single electronic rotation responsible for the eventual generation of the QMA's saddle field. ${ }^{58}$ It is common practice to describe ionic trajectories in terms of vectors, by convention $\mathrm{X}$ and $\mathrm{Y}$ describe the ions location relative to the cross-section of the electrode array while $\mathrm{Z}$ represents the distance the ion has traveled down the length of the analyzer. To aid in the conceptualization of this electromagnetic phenomenon the X$\mathrm{Y}$ and $\mathrm{X}-\mathrm{Z}$ influences will be considered separately, also, an attempt will be made to separate the time-dependant (alternating) and time-independent (direct) electronic influences into their principal components.

When an $\mathrm{AC}$ voltage is applied to an electrode pair, in the case the potential will be applied to the $\mathrm{X}$ electrodes thereby influencing the trajectories of ions in an $\mathrm{X}-\mathrm{Z}$ orientation, each $\mathrm{X}$ electrode will spend half of the cycle positively charged while the opposite electrode conveys a negative potential. ${ }^{59}$ In a conventional quadrupole, each of the two electrode pairs $(\mathrm{X} \& \mathrm{Y})$ remain $180^{\circ}$ out of phase at all times, in this way when a positive potential is conferred to one electrode, an identical negative charge is conferred

*While an effort has been made to introduce the mathematical basis of instruments until this point, it would not be reasonable to introduce the advanced differential equations governing the operation of the QMA given the already considerable length of this chapter. For the concerned reader a number of articles are readily available which discuss the quantum-mechanical principles of the quadrupole array in depth, including an excellent piece by Philip Miller at the University of Arizona. ${ }^{60}$ 
to the electrode spatially opposite. Should positively charged ions be introduced during this electronic cycle, again only considering travel in the $\mathrm{X}-\mathrm{Z}$ plane relative to the center axis of the array, positively charged ions experiencing a net positive AC potential will be repelled (focused) towards the center of the quadrupole. ${ }^{61}$

As the array exists in phased equilibrium, the inverse is also true, when a negative potential is expressed the positive ions will be attracted towards the negatively charged poles, effectively defocusing the ion beam. ${ }^{62}$ Should the defocusing interval be sufficient in duration, the positively charged ions will eventually collide with the negatively charged electrode and be excluded from the ion beam. ${ }^{63}$ In this way the ionic trajectories through a QMA may be influenced both by the perturbation of the electronic potentials expressed by the electrodes and by the duration of those potentials.

The Y-plane, time independent DC electrodes serve to compliment the focusing action of the X-plane to perturb the flight of ions in a mass dependent fashion. ${ }^{64}$ When the alternating and direct potentials are evaluated in concert with time, the mass focusing properties of the QMA began to become evident. Should an amalgam of ions be introduced into the array, the light and heavy species would interact with the electronic gradients quite differently. If an ion is quite heavy, its resultant momentum (trajectory) will be largely unaffected by rapid perturbations of the electronic gradient and the ion will only feel the net effect of the electric potential.

Due to the conservation of momentum, heavy ions tend only to be effected by time independent forces. ${ }^{65}$ Conversely, very light ions will be significantly perturbed by very rapid (time-dependent) oscillation of the electric gradient in the array. In fact, small ions may be accelerated rapidly enough to exit the ion beam entirely and collide with the oppositely charged electrode, thus being effectively mitigated from detection. In this way a QMA is capable of excluding ions above or below a critical value based upon the speed at which an ion can respond to the defocusing potential exerted upon it. ${ }^{66}$ The undesirable ions are allowed to collide with the electrodes and be excluded from the ion beam while ions of a desired mass are allowed to traverse the array and be counted by the detector. ${ }^{67}$

Many modern analytical laboratories now rely on the implementation of hyphenated or hybrid mass spectrometers. ${ }^{68}$ In this way, scientists are able to overcome many of the physical limitations inherent to a single spectrometer type. While in no way a modern implementation, the quadrupole-TOF remains the centerpiece of many small molecule and proteomic laboratories alike and is responsible for a great deal of the data outlined in this work.

\section{Introduction to Chromatography}

The term "Chromatography" has come to be synonymous with numerous laboratory techniques employed in the separation of mixtures. ${ }^{69}$ In the most generic of terms, the analyte of interest is introduced into a liquid (LC) or gas (GC) mobile phase which serves to transport the sample through a structurally constrained stationary phase. 
The analyte is then separated into its principal components as some compounds are capable of migrating through the stationary chemistry more rapidly than others. ${ }^{70}$ The speed at which an analyte achieves equilibrium between chromatographic phases and is ultimately eluted from the chromatographic column (retention time) is largely a function of a compounds physical-chemical properties, which in the case of separation sciences, is often expressed as a partition coefficient. ${ }^{71}$

\section{Gas Chromatography}

A typical gas chromatograph consists of a carrier gas source, a sample injector, a column (contained in an oven) and some means of detection. ${ }^{72}$ While derivatization techniques exist, analytes are generally considered to be $\mathrm{GC}$ compatible if they possess a relatively low boiling point $\left(<250^{\circ} \mathrm{C}\right)$ and they do not tend to undergo thermal decomposition (non-thermolabile).$^{73}$ In GC the analyte is dissolved into a low-boiling solvent (such as $\mathrm{CH}_{3} \mathrm{OH}$ ) and is introduced to a heated injector. Upon injection the solvent is immediately evaporated yielding an injection chamber filled with nebulized analyte and residual injection solvent. ${ }^{74} \mathrm{~A}$ carrier gas (mobile phase) then transports the vaporized analyte/solvent matrix into the chromatographic column housed in a high temperature oven. The column serves to structurally support the chromatographic stationary phase to which the analyte/solvent matrix is introduced at the column head. ${ }^{75}$ Two types of oven programs are often used to achieve analytical separation; isothermal and thermal gradient. In an isothermal program the oven (and the column it contains) are preheated to a temperature at which the analyte of interest will be retained (equilibrate with the stationary phase) while the solvent matrix will be virtually unretained and eluted rapidly. ${ }^{76}$

In the case of a thermal gradient, the oven is set to a sufficiently low temperature to allow the analyte/solvent matrix to initially equilibrate with the stationary phase. The oven is the heated in a controlled fashion with the solvent eluting first followed by the analytes of interest, the use of a thermal gradient often aids in the mitigation of solvent diffusion effects often seen in isothermal runs however at the cost of increased complexity. ${ }^{77}$ As the analytes are eluted from the column, the elutant is directed to some form of detector.

The fifty plus year history of GC has seen numerous detection methodologies, though today most pharmaceutical laboratories often rely primarily on a combination of three, ${ }^{78}$ Flame Ionization Detector (FID), Electron Capture Detector (ECD) and MS. As MS is the focus of the work only FID and ECD will be discussed briefly here.

\section{Flame Ionization Detector}

Today FID has established itself as a popular and robust detection method; it relies upon the ability of a hydrogen/oxygen (air) flame to ionize organic molecules. These ions are then directed across an adjacent electrode, the resultant ion current is 
directly proportional to the ions generated per unit time. ${ }^{79}$ The FID has a number of endearing qualities; very low cost, minimal maintenance, no radionuclide, exceptional linearity and dynamic range and a very reasonable sensitivity for carbon containing compounds. ${ }^{80}$ For these reasons the FID has established itself as a near universal GC detector, with many laboratories still relying on it heavily to this day. ${ }^{81}$

\section{Electron Capture Detector}

While much less common than the FID, the ECD enjoyed a significant success throughout the history of GC. ${ }^{82}$ The ECD relies on the use of a $\beta$ radiation source $\left({ }^{63} \mathrm{Ni}\right)$ and offers exceptional sensitivity in the detection of halogenated compounds. ${ }^{83}$ In an ECD $\beta$ particles are emitted into a makeup chamber filled with a continuous supply of nitrogen. ${ }^{84}$ The low excitation energy of nitrogen allows the $\beta$ particle to easily strip a valence electron; these electrons collide with additional nitrogen atoms stripping additional electrons resulting in a $\beta$ particle induced electron cascade. These many electrons are then attracted towards a positively charged anode generating an electric potential. As the elutant is introduced into the detector, the analyte effectively disrupts the flow of electrons and reduces the electric potential between the ECD's cathode and anode. This loss of voltage is proportional to the concentration of analyte in the detection chamber at any given time. The ECD has been relied upon heavily in the past for the detection of nitro, nitrile and halogenated compounds. While the ECD will likely remain significantly utilized for the foreseeable future, regulatory difficulties arising from the ECD's reliance of radionuclides have led many laboratories to transition from ECD to MS based instrumentation. ${ }^{85}$

\section{Liquid Chromatography}

The term "liquid chromatography" (LC) has come to encompass many different separation techniques and technologies. Numerous LC methodologies have been utilized in concert with mass spectrometry over the years, ${ }^{86}$ however none have had the impact of high-performance liquid chromatography ${ }^{87}$ (HPLC). HPLC/MS has proven to be an extremely powerful tool in the analysis of the pharmaceutical compounds. ${ }^{88,89}$

The typical HPLC consists of a one or more high pressure liquid pumps, a sample injector (manual or automated) a chromatographic column (often contained in an oven) and ultimately a means of detection. ${ }^{90}$ In this way the generalized schematic representation if an HPLC is itself very similar to the GC before it. As the name implies, the greatest contrasts between the modern HPLC and modern GC is the mobile phase they employ and the means by which they ultimately achieve separation. While the GC relied on a single gas to carry the analyte, the modern HPLC relies on a system of pumps (minimally binary, often quaternary) to deliver a precisely mixed mobile phase. ${ }^{91}$ The ability of an HPLC system to accurately dispense these solvents proves critical to the robustness of the instrument. ${ }^{92}$ In the GC, the limited heat capacity of the gas coupled with the low mass of the chromatographic capillary allowed separation to be repeatably 
achieved by simply varying the temperature of the column oven.${ }^{93}$ While many HPLC instruments are now equipped with column ovens themselves, their function is more to mitigate variations in laboratory temperature than to singularly achieve separation.

While variations on the classical methods exist, the HPLC generally achieves separation through the use of a compositional gradient of a polar (often $\left.\mathrm{H}_{2} \mathrm{O}\right)$ and organic mobile phase. ${ }^{94}$ Most modern laboratory implementations of HPLC/MS for the analysis of sample purity or composition begin with the analyte of interest being dissolved in a small volume of compatible solvent ${ }^{95}\left(\mathrm{H}_{2} \mathrm{O}, \mathrm{CH}_{3} \mathrm{OH}, \mathrm{ACN}\right)^{*}$. The chromatographic column, containing the stationary phase, is allowed to initially equilibrate (both thermally and compositionally) and the sample is introduced into a highly aqueous matrix. Over the duration of the chromatographic run the composition of the mobile phase is slowly ramped from a highly aqueous to that of a highly organic nature. ${ }^{96}$ During this process, components of the analytical amalgam which were previously retained on the stationary phase slowly become more compatible with the mobile phase, partition, proceeded towards chromatographic equilibrium (begin migration) and are eventually eluted from the stationary phase as separate species. ${ }^{97}$ These distinct chemical species are then detected or analyzed (MS) to ultimately evaluate the effectiveness of the chromatic process.

While in many ways the MS has become the most informatically rich detector available to the typical analytical laboratory, most HPLC systems are equipped with detection strategies complimentarily orthogonal to spectrometry. As many laboratories are limited by both available instrument time and sample quantity many instrument manufactures have made significant strides to provide systems capable of utilizing multiple detection strategies during a single run.

\section{Ultra-Violet/Visible Detector (UV/Vis)}

$\mathrm{UV} / \mathrm{Vis}$ is in many ways the most obvious compliment to the MS due to the nondestructive nature of this detector. In a typical UV/Vis, the chromatographic elutant enters the detector's transparent flow cell where it is exposed to ultraviolet or visible irradiation at a known wavelength. ${ }^{98}$ Analytes which contain $\pi$ or non-bonding electrons are able to absorb energy through the transitioning of electrons to higher anti-bonding molecular orbitals. ${ }^{99}$ The absorption of energy can be recorded and spectra generated, in concert, UV and MS spectra have proven to be a powerful tool in the analysis of pharmaceutical compounds.

*As this introduction is largely devoted to mass spectrometry, reverse phase HPLC will be introduced as it is vastly more compatible with traditional MS instrumentation than other conventional elution systems. 


\section{Evaporative Light Scattering Detector (ELSD)}

While ELSD is a destructive analytical technique, it has gained great popularity for its ability to evaluate many compounds which typically ionize poorly via MS. ${ }^{100}$ The ELSD is also somewhat unique in its ability provide a relatively linear signal response independent of the optical properties of the molecule analyzed, in contrast the $\mathrm{Uv} / \mathrm{Vis}$ was only compatible with analytes that contained an accessible chromophore. ${ }^{101}$ When compared to a MS, the ELSD is quite simplistic. The elutant flow is introduced into the ELSD nebulizer and mixed with large volumes of nitrogen gas to produce a diffuse plume of effluent. The plume is then directed down a heated evaporation tube where the remaining mobile phase is rapidly eliminated, leaving only diffuse particles of dried analyte. ${ }^{102}$ The particles of dry analyte are directed through an optical chamber where they occlude a high intensity light source. This loss of signal can be recorded and spectra generated much as is the case with the Uv/Vis. 


\section{CHAPTER 2. HRMS BASED INVESTGATION OF BACTERIAL SECONDARY METABOLITES}

\section{An Introduction to Bacterial Secondary Metabolites}

Like their human hosts, pathogenic bacteria utilize a system of complex strategies by which they obtain the nutrients and energy necessary for survival and reproduction. Most bacteria assimilate the organic molecules they require to thrive from their environment. In this way pathogenic bacteria reap organic molecules from their host organism, consume the portion they require, and secrete the waste byproducts as primary metabolites. ${ }^{103}$

By contrast, secondary metabolites are compounds produced not as a function of survival, but as a function of competition. The list of known bacterial secondary metabolites has become quite long, and have been used both primarily and as synthetic scaffolds for; antibiotics (penicillin), pigments, immunomodulators (of which this chapter is concerned), pesticides (pyrethrins) and antitumor agents. ${ }^{104}$ Two pathogenic microorganisms of particular interest to our laboratory are Francisella tularensis and Mycobacterium ulcerans (and will be introduced in detail later in this chapter.)

\section{Decoupling of Nondestructive (NMR) and Destructive (MS) Analytical Techniques}

While this chapter serves to illustrate two pathogens of particular concern to our laboratory, the overarching goal of our bacterial natural product (BNP) effort was not solely to investigate $F$ tularensis and Mulcerans. The primary focus of these efforts was to establish a universal means of profiling unknown bacterial natural products regardless of their species of origin. The resulting workflows were designed in such a way as to leverage nondestructive analytical techniques, such as crude extract NMR, to enable the targeted application of destructive, more informatically rich techniques. The initial proton and 2D crude lipid NMRs coupled with sample conserving analytical TLC provide information on the chemical species contained in the BNP while selective TLC developing agents, such as Sigma Phospray, can support and enumerate NMR results.

In this workflow data acquired through minimally invasive techniques can target the application of HPLC or Flash chromatographic fractionation as well as conventional UPLC/MS analysis depending on the quantity of sample available for analysis

(Figure 1). In the case of SchuS4, its highly virulent nature precluded the acquisition of large analytical stocks. For this reason our workflow was updated to allow for the pooling and serial concentration of analyte limited products (Figure 2). Once sufficient quantities of analytical samples have been compiled, High Content NMR experiments (HSQC, HMBC, COSY, NOESY) coupled with HRMS accurate masses can be applied to the enumeration of potential chemical formula For this reason analytical techniques in this section will be presented in order of chemical invasivity from the least destructive (NMR) to the most destructive (FAME) techniques following our BNP protocols. 


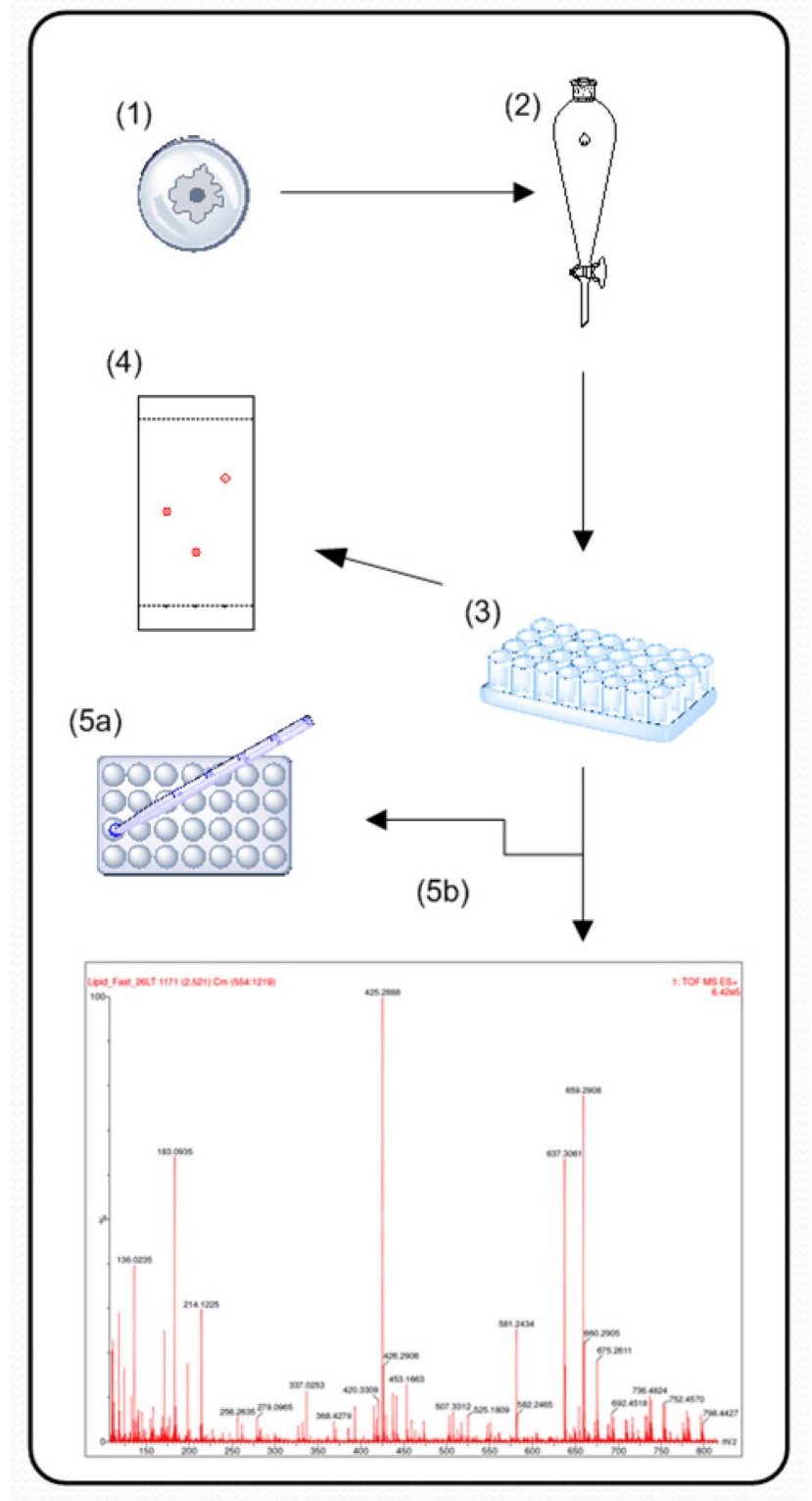

Figure 1. Pictorial Representation of SchuS4 BNP Analytical Workflow Illustrating the Extraction, Separation and Analysis of Bacterial Natural Products

NOTES: SchuS4 / LVS were harvested and acetone soluble lipids (ASL) were removed and dried. The dry ASL was redisolved in $\mathrm{MeOH}$ and deactivated silica $60 \mathrm{~A}(1 \mathrm{~g})$ added, the resulting mixture was then evaporated to dryness via rotovap, the lipid impregnated silica was transferred to the top of a $4 \mathrm{~g}$ HP-Sil (Biotage) cartridge for flash chromatography using a eluent gradation of $90 \%$ CHCL3/ $10 \% \mathrm{CH}_{3} \mathrm{OH}$ to $60 \% \mathrm{CHCL} 3 /$ $40 \% \mathrm{CH}_{3} \mathrm{OH}$, all fractions were collected and content monitored via by TLC analysis. The lipids potential for immune modulation of each fraction was bio-assayed, fractions retaining biological activity were further analyzed using UPLC-qTOF. 


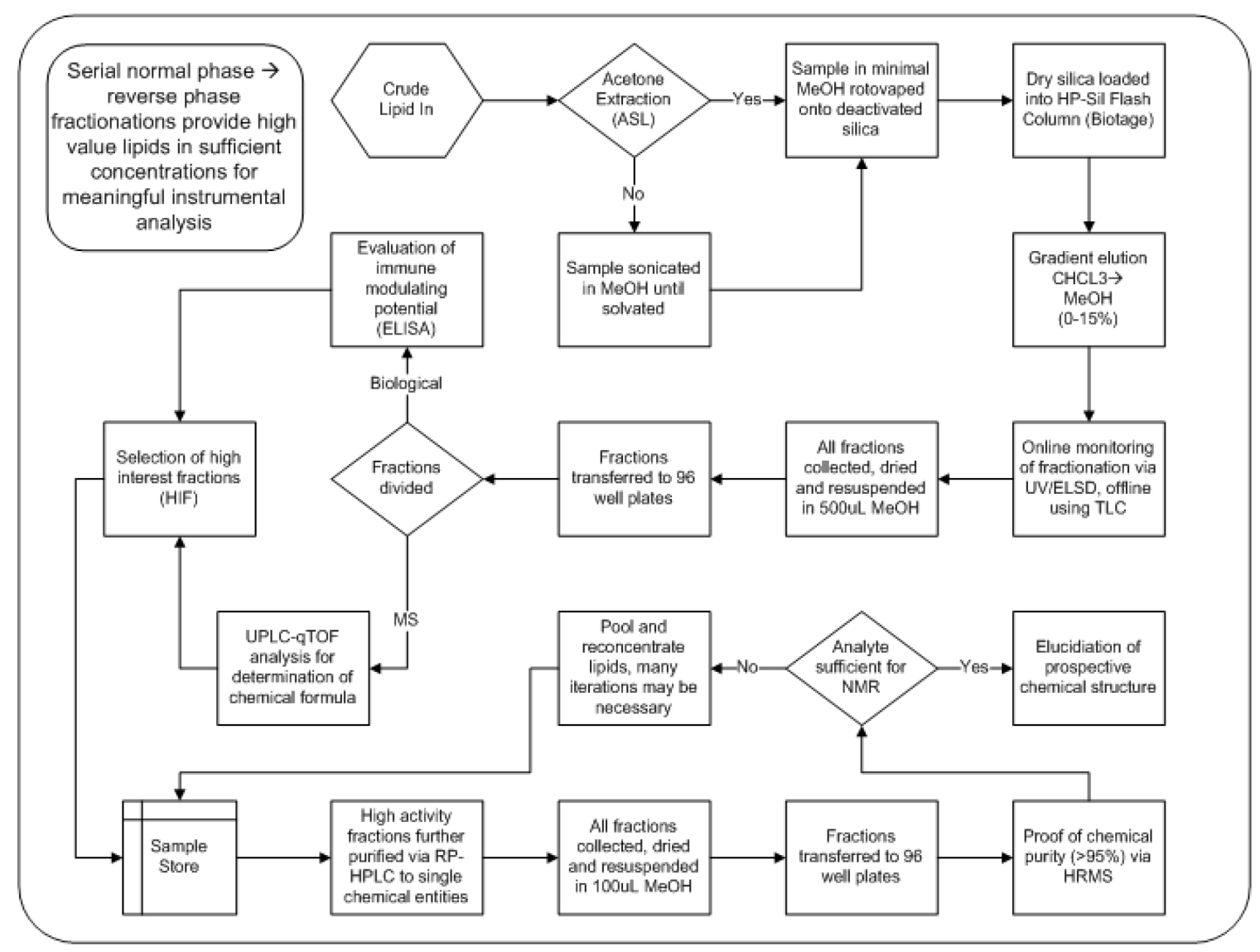

Figure 2. Detailed Workflow for the Separation and Analysis of Product Limited Francisella tularensis Extracts

NOTE: The addition of an offline sample store allows archival of analytical stocks until sufficient concentrations have been achieved through serial fractionation and pooling efforts. 


\section{Introduction to Francisella tularensis}

$F$ tularensis $(\mathrm{Ft})$ is a pathogenic gram-negative bacteria which causes the disease Tularemia in humans. As the pathogen is readily transmissible by aerosol and highly virulent it has been classified as a Class A Select Agent in the United States. Ft is also unique from a metabolic tractability standpoint as the virulent SchuS4 strain and nonvirulent live vaccine strain (LVS) appears to retain very similar (though distinct) lipid profiles. Recent work in the Bosio Laboratory at the National Institutes of Health has shown lipid extracts from FT when administered to stimulated murine bone marrow derived microphages (BMM) significantly attenuated the inflammatory cytokine response. This attenuation suggested that the extracts from the SchuS4 strain contained an immune suppressive substance. Interestingly, Ft lipids have also shown to elicit protective immunity in MyD88 -/- mice through the surface expression of Lipid A analogs. ${ }^{105}$ When separated into a ternary system via modified Folch ${ }^{106}$ partitioning, the majority of the attenuating agent remains in the chloroform fraction, supporting the hypothesis that SchuS4 lipids are important virulence factors (Figure 3).

Preliminary testing revealed the lipids from the virulent Ft SchuS4 strain displayed a significant affinity for cytokine attenuation while similarly prepared lipids from the attenuated Ft live vaccine strain (LVS) showed little to no effect. Initial samples of dried lipid were suspended in $\mathrm{CDCl}_{3}$ and submitted for proton NMR (Figure 4). Small lipid peaks may be resolved but the crude lipid was found to be highly contaminated with bacterial cellular debris, 2D NMR also proved quite complex (Figure 5). The crude lipid was separated via 2D TLC as well as fractionated by standard phase flash chromatography in an attempt to reduce background contamination. A portion of the fractionated materials were returned to NIH for testing, the areas of high activity were pooled and further analyzed via LC/MS. Traditional and 2D TLC's of Schus4 and LVS lipid crudes were run using a semi-analytical technique (Figure 6) the bioattenuation properties were then evaluated (Figure 7), this data provided us the ability to locate the "most active" components for further "destructive" analysis.

Guided by cytokine deactivation potential of SchuS4 (Figure 8) as compared to the LVS profile (Figure 9), fractions were concentrated into three pools. As fractions were separated via normal phase silica gel chromatography the pools were generated based upon the polarity of the analyte. The highly nonpolar (Fractions 4-5), the moderately polar (Fractions 19-23) and the most polar (Fractions 28-34) pools were then submitted for further analysis. (Figure 10) Following the BNP workflow lipid fraction pools were first analyzed via nondestructive techniques (NMR, UV). These data were then used to guide the application of more intrusive chemical analysis (HPLC/MS)(GC/MS). Processed NMR and UPLC/MS spectra of the nonpolar pool are illustrated below. Figure 11 is a processed spectra from 1024 scan proton NMR. After primary fractionation the spectra remains complex though appears to contain significant concentrations of saturated lipids (1-2 ppm) and reduced concentrations of unsaturated lipid products (4.0-4.7 ppm). Figure 12 illustrates a processed 2D HSQC of this sample, when compared to the unfractionated sample the fatty acid and phospholipid regions appear to have increased relative to the aromatic component. 


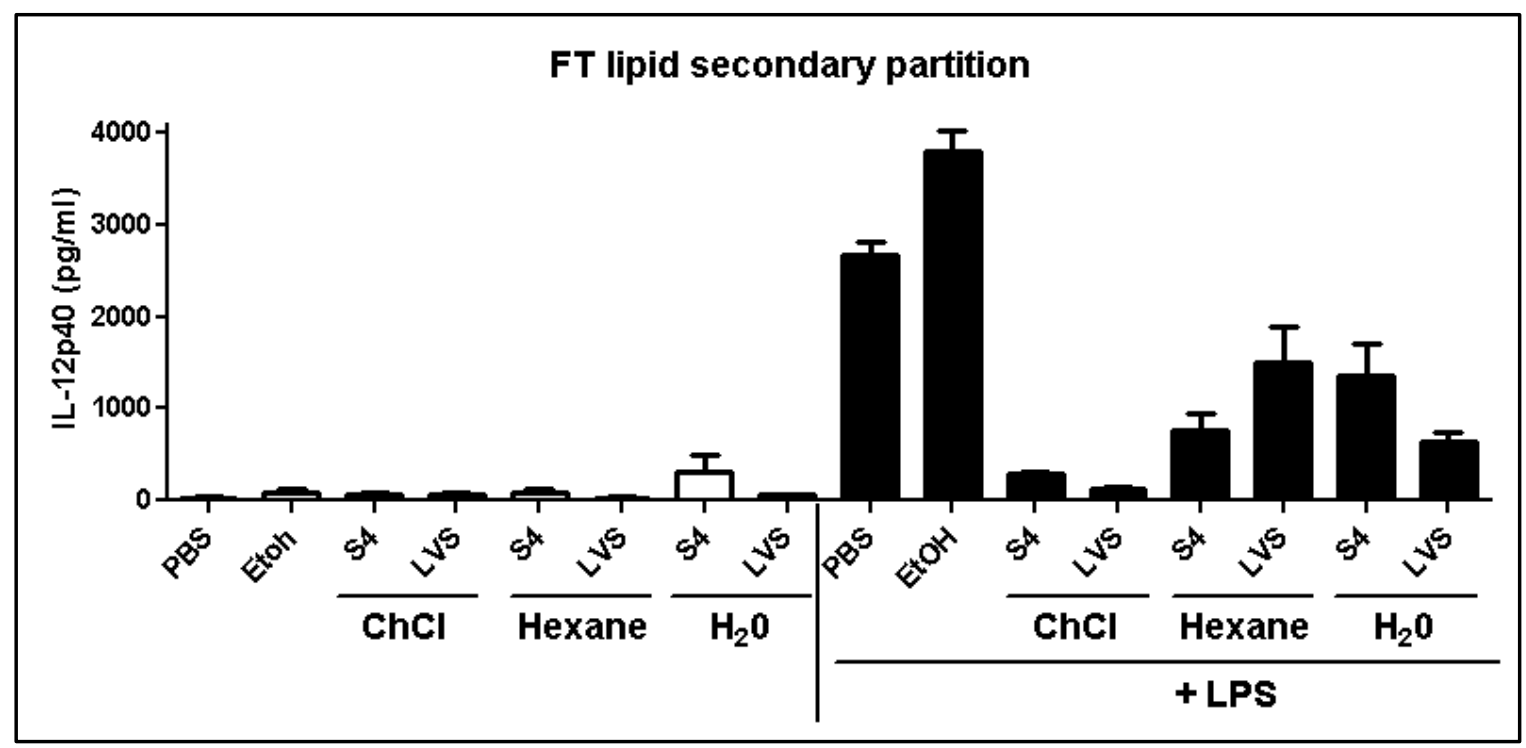

Figure 3. Activity of Modified Folch SchuS4 Partitioned Lipids with and without Addition of the Cytokine Activating LPS, with the Chloroform Fractions Predominately Responsible for Cytokine Attenuation 


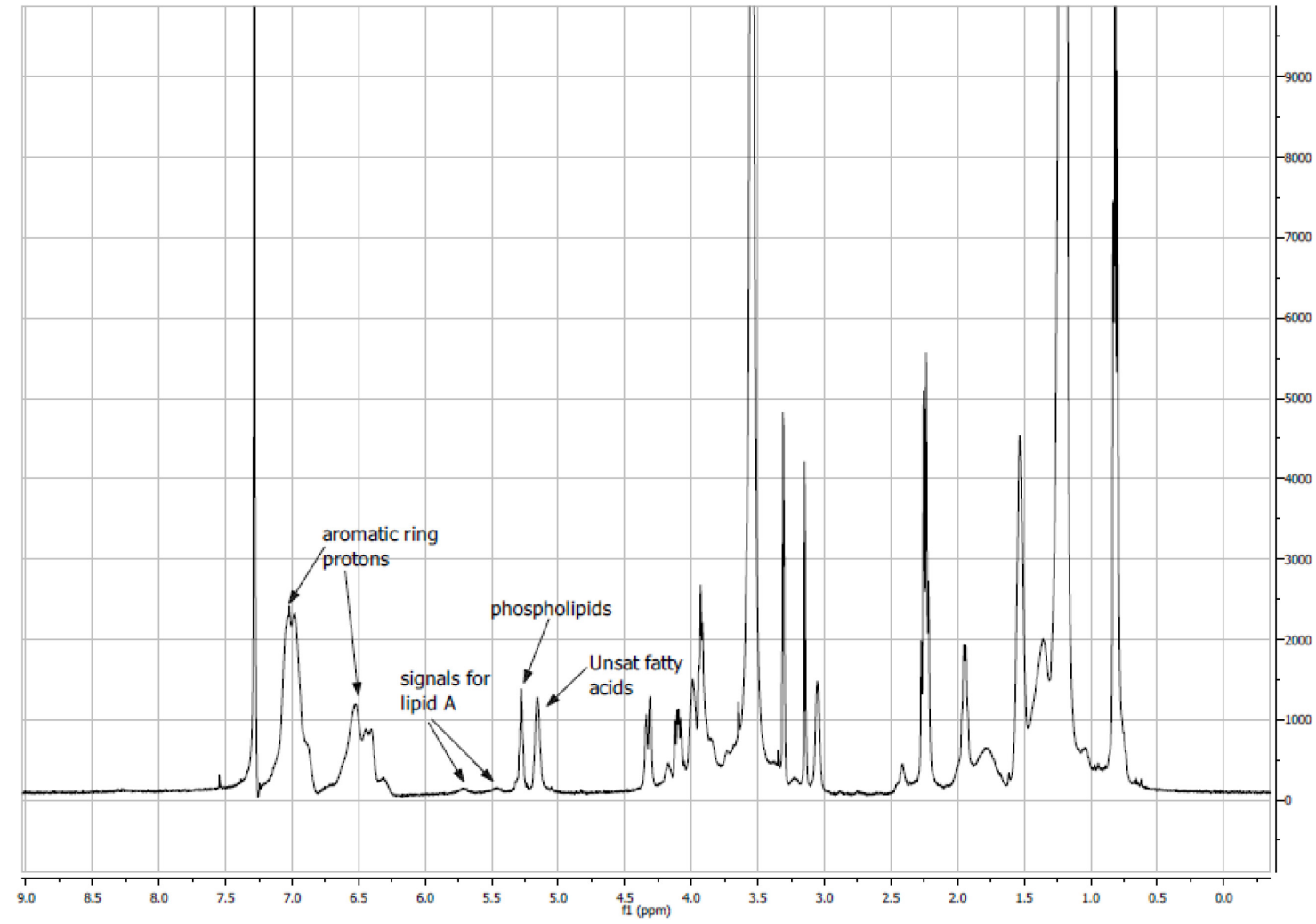

Figure 4. Complex Proton NMR Spectra of Crude SchuS4 Lipid Extract with Lipid Signals Largely Masked 


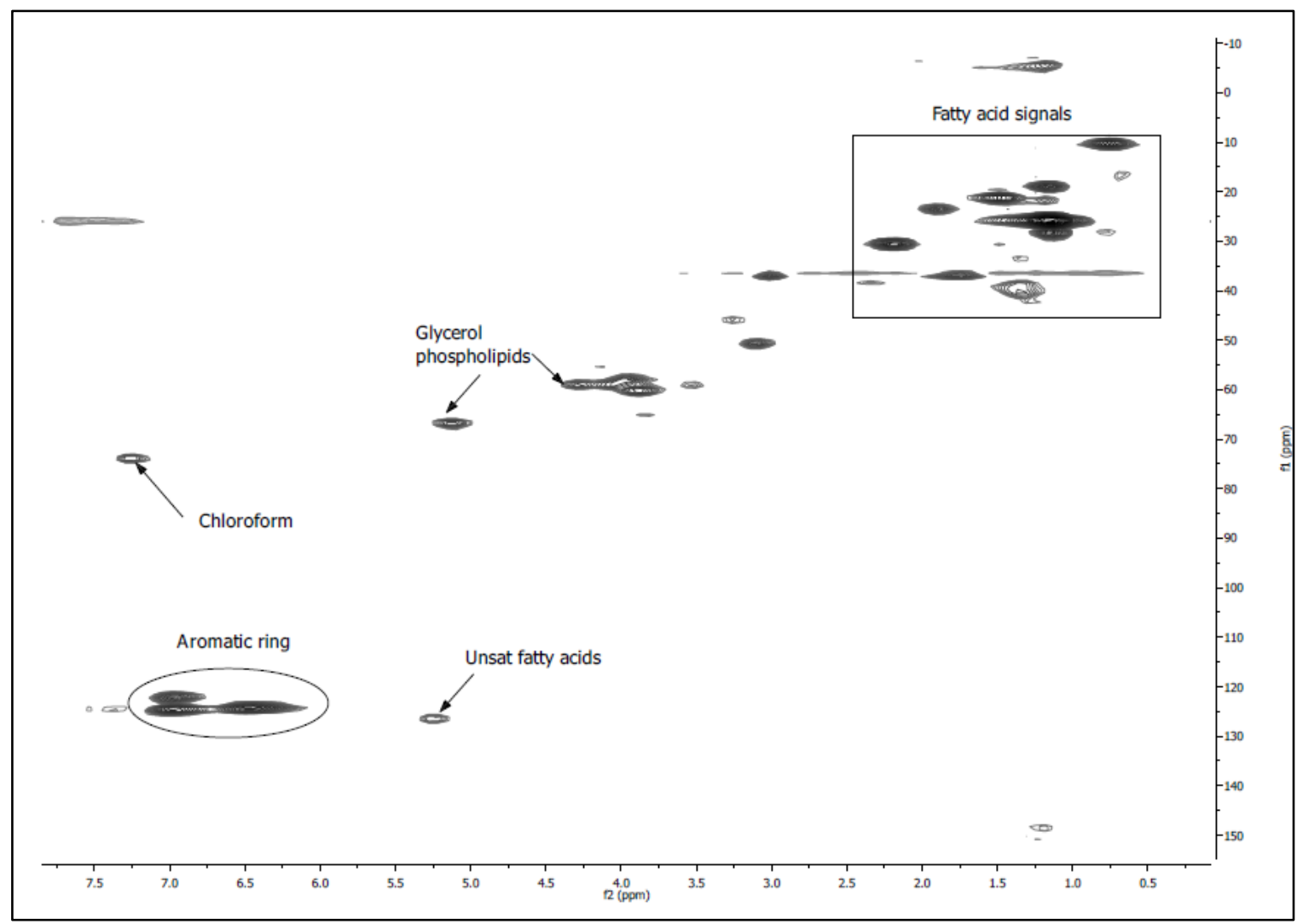

Figure 5. Complex 2D NMR of Crude SchuS4 Lipid Sample

NOTE: The fatty acid region remains highly complex. 


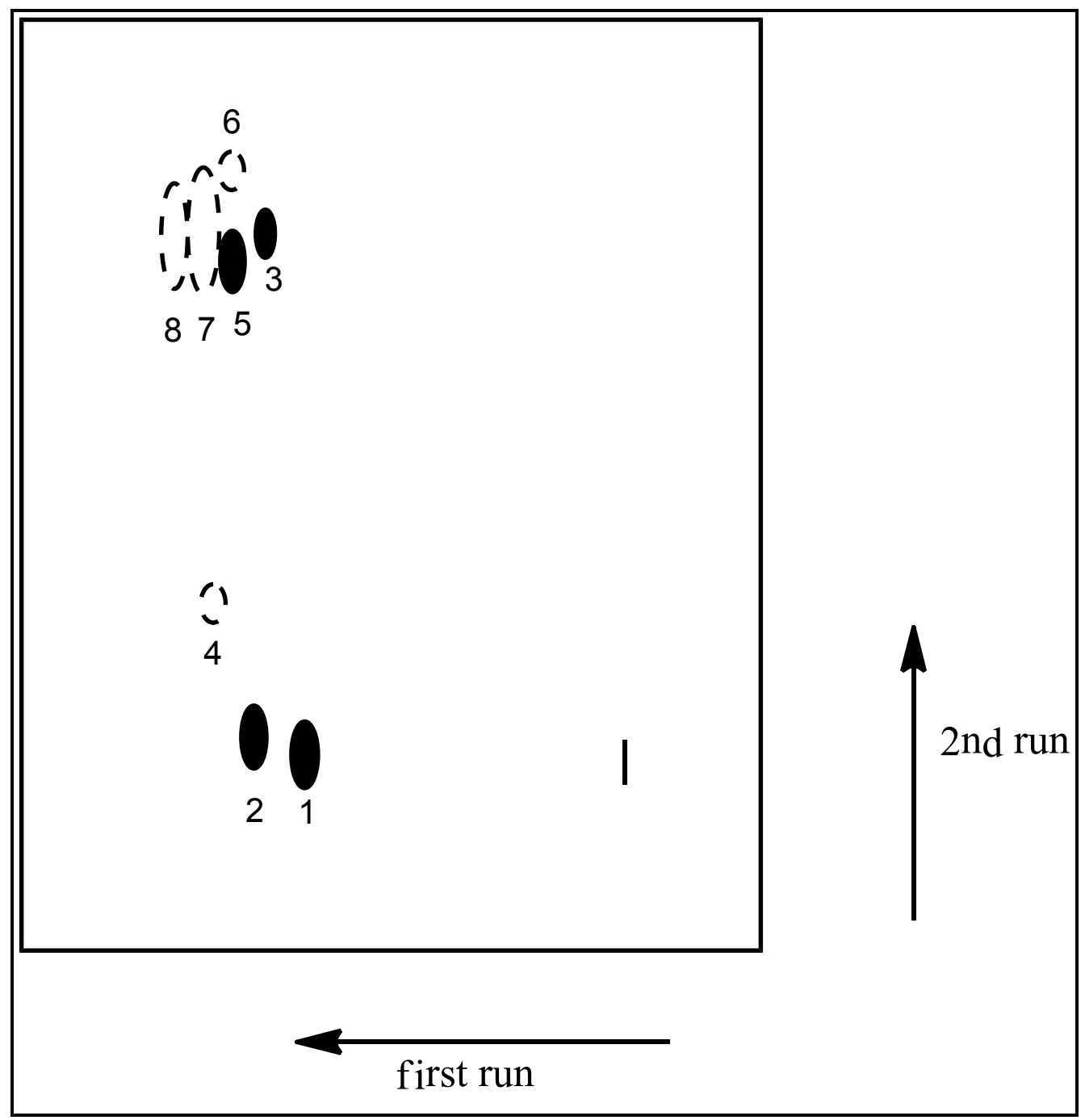

Figure 6. Illustration of Typical SchuS4 Lipid 2D TLC

NOTES: Black line indicates the point of origin; the analytes were first eluted in $(12: 6: 1)$ $\mathrm{CHCl}_{3}: \mathrm{MeOH}: \mathrm{H}_{2} \mathrm{O}$. The plate was rotated $90^{\circ}$ and again separated via (10:6:4:1) THF:1,2-Dimethoxymethane:MeOH:1 $\mathrm{M} \mathrm{NH}_{4} \mathrm{OH}$. 


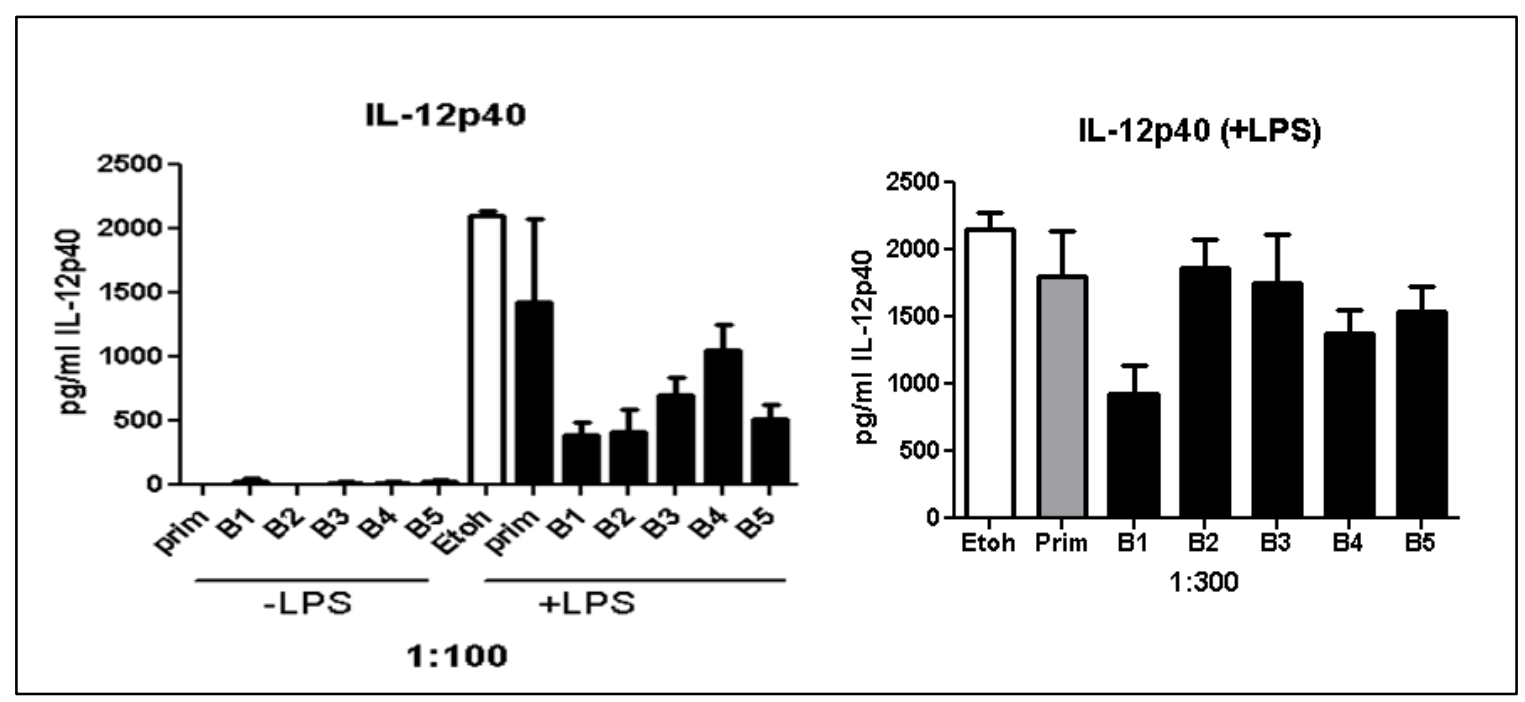

Figure 7. Bioanalysis of SchuS4 2D TLC Bands (B1-B5) at 1:100 and 1:300.

NOTE: Dilutions indicate TLC Band 1 retains significant cytokine attenuation potential in primary murine BMM cells. 


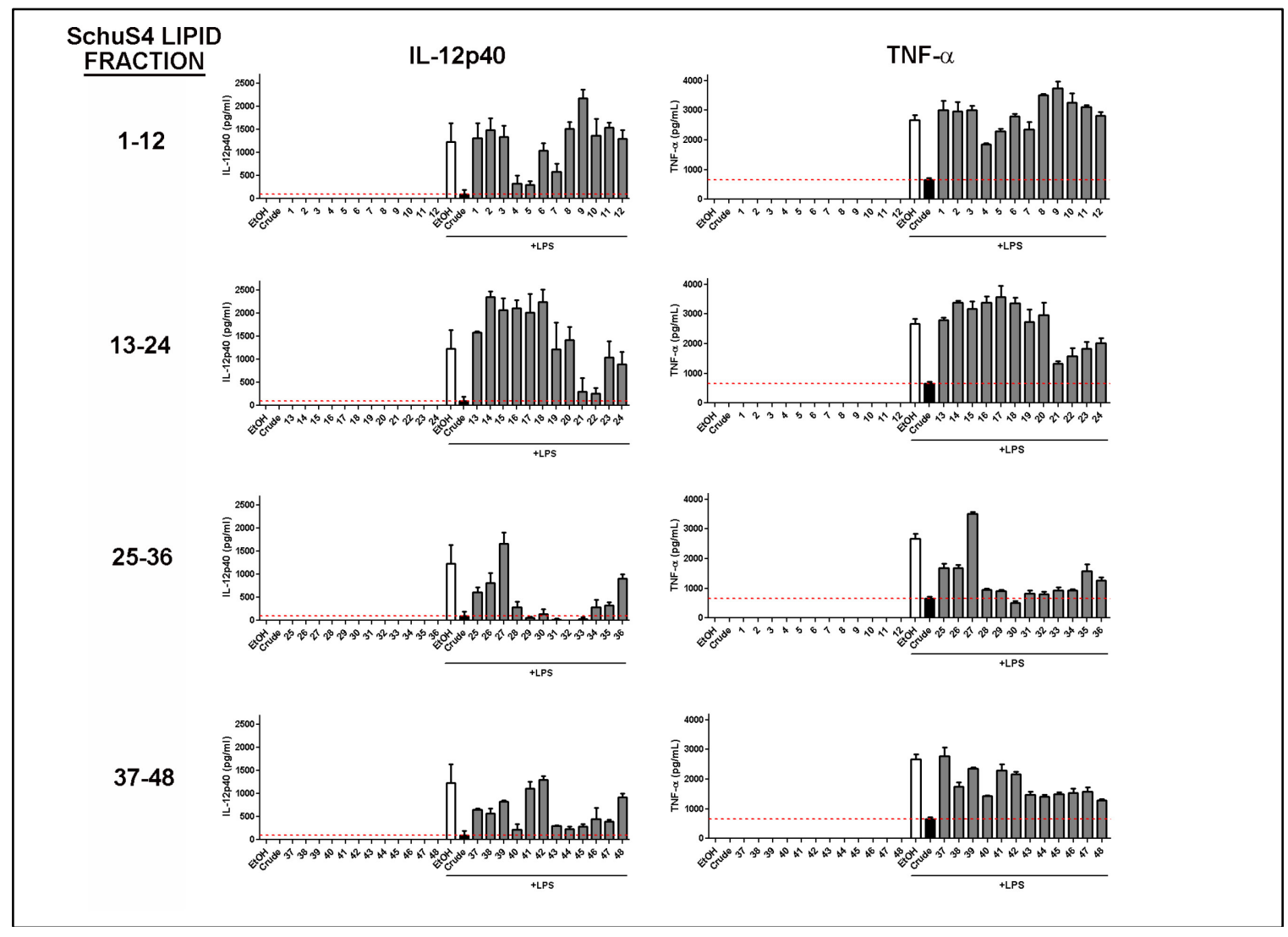

Figure 8. Cytokine Deactivation Potential of Initial Flash Chromatographic Fractions of SchuS4 Lipid Extract 


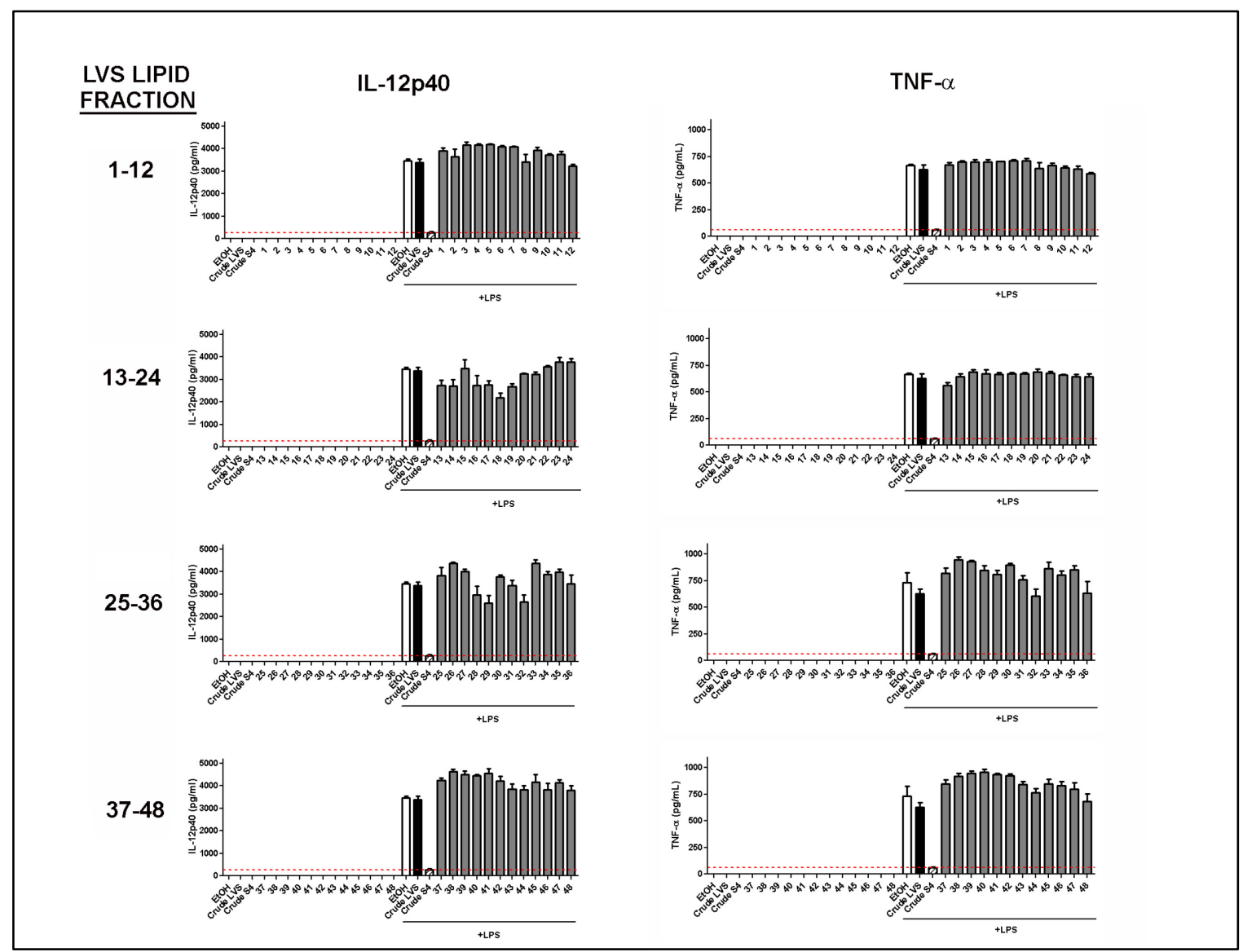

Figure 9. Cytokine Deactivation Potential of Initial Flash Chromatographic Fractions of LVS Lipid Extract 


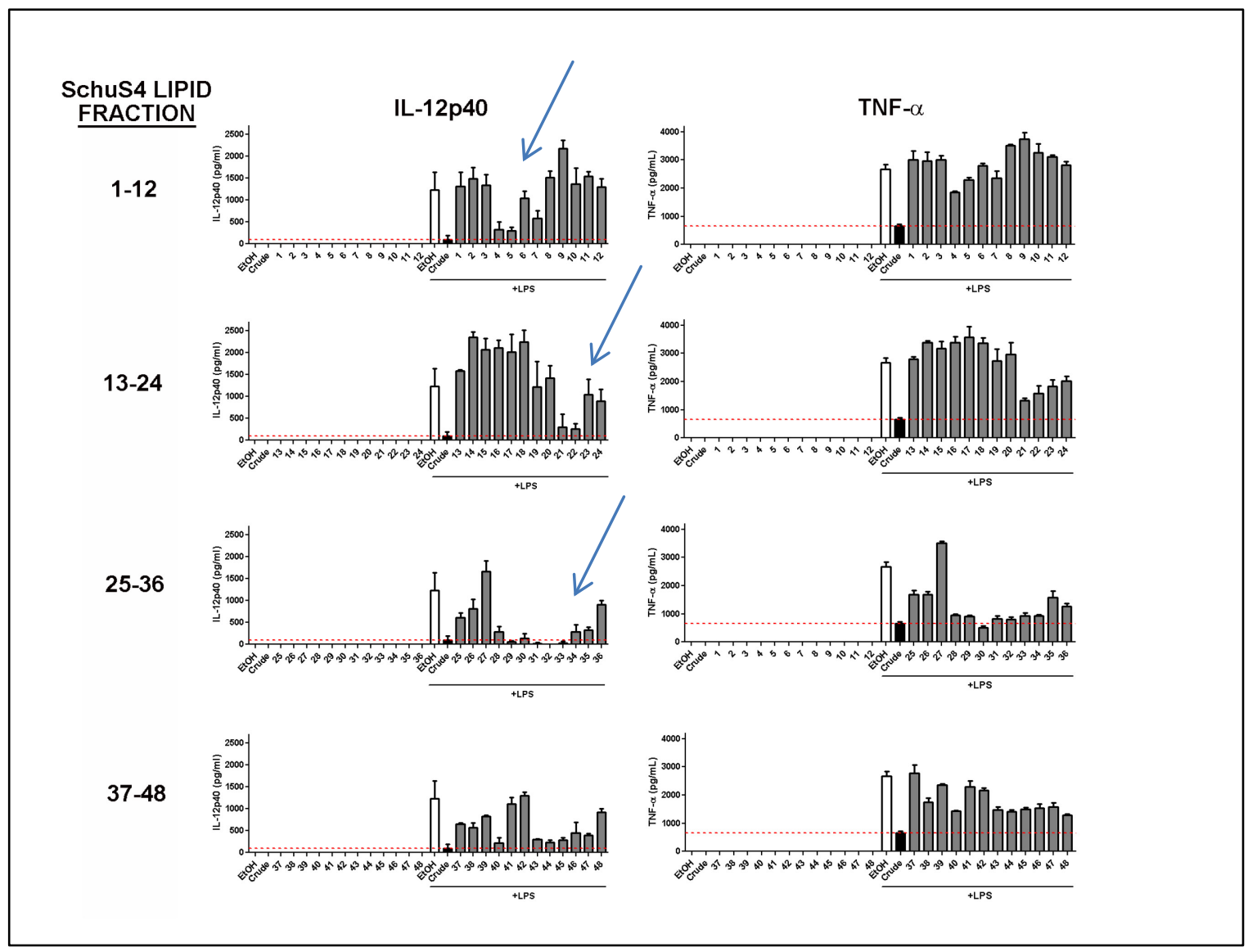

Figure 10. Cytokine Deactivation Potential of Initial Flash Chromatographic Fractions of SchuS4 Lipid Extract Illustrating Three Areas of Activity

NOTE: Fractions 4-5, 19-23 and 28-34 were pooled for further analysis. 


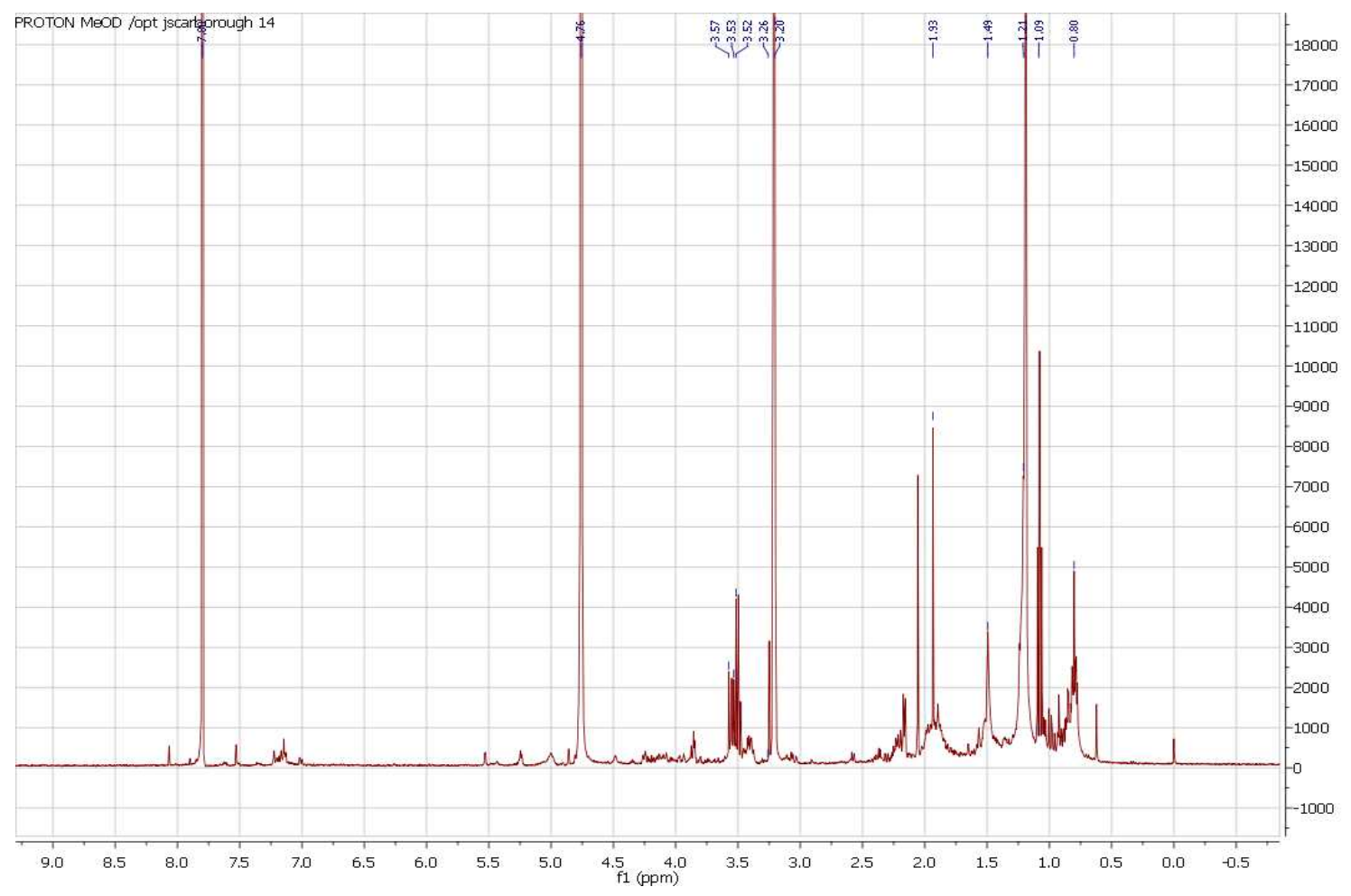

Figure 11. 1024 Scan Proton NMR of Pooled SchuS4 Fractions 20-24

NOTE: The saturated and unsaturated regions of the spectra remain highly complex. 


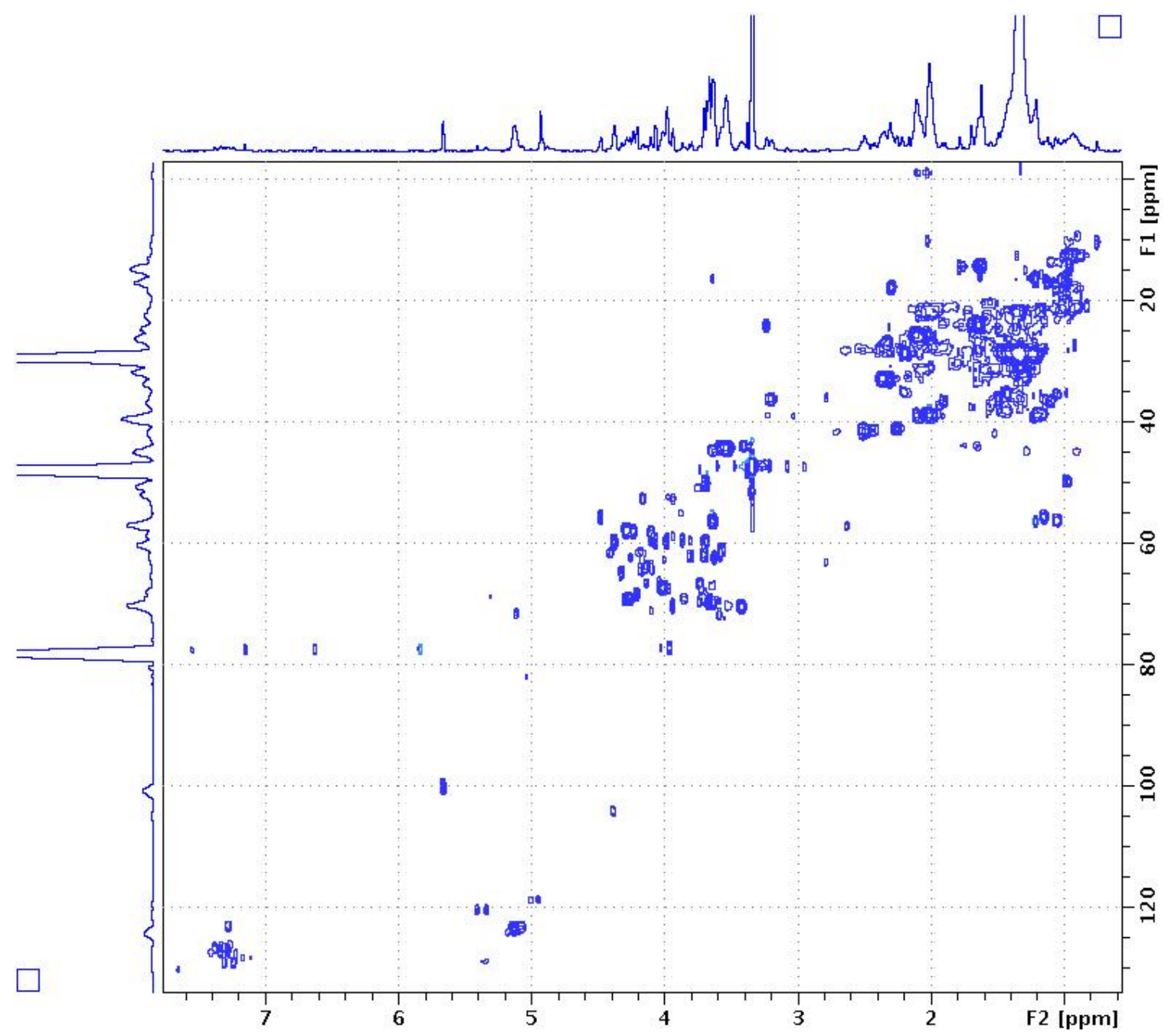

Figure 12. 2D HSQC of Pooled SchuS4 Fractions 20-24 Depicting the Enrichment of Fatty Acids and Phospholipids 


\section{Fatty Acid Methyl Ester Derivatization and GC/MS Analysis}

As many of these experiments predated our laboratories acquisition of a HRMS, Figure 13 depicts a processed UPLC-SQD spectrum of the concentrated nonpolar lipid pools of LVS and ScghuS4 lipid. The peaks at $581.2 \mathrm{~m} / \mathrm{z}$ (butylstyrene) and $463.3 \mathrm{~m} / \mathrm{z}$ (polypropylene)(Figure 14) appear to be highly significant but were later determined to be common laboratory contamination. Additional analysis revealed lipids were likey present in the very polar pool however they appeared to be poorly ionizable and similarly contaminated via ESI-LC/MS (Figure 15). At this point in the investigation a decision was made to temporally discontinue the use of LC/MS in favor a of GC/MS based fatty acid methyl ester (FAME) workflow as the derivatization process ( $\mathrm{HCl}-\mathrm{MeOH}$ or $\mathrm{KOH})$ would largely disregard the observed laboratory contamination.

Fatty Acid Methyl Esterification GC/MS analysis enabled direct evaluation of SchuS4 and LVS matched acetone soluble lipid (ASL) crude lipid samples without the sample diminishing chromatographic step. Methyl esters of the ASL lipid crudes were prepared by transesterification using $\mathrm{HCl}-\mathrm{CH}_{3} \mathrm{OH}$ or base catalyzed ( $\mathrm{KOH}$ or TBAH) iodomethane methylation. The resultant spectra were analyzed by GCMS using a BD-225 column and 42 minute thermal gradient from $90^{\circ}$ to $220^{\circ} \mathrm{C}$. Elutant peaks were compared to NIST spectral database for peak identification. Upon comparison the SchuS4 and LVS spectra are quite similar (The LVS Peak 8 tailing is due to the aforementioned preexisting contamination) except for the large decanoic acid peak (2) completely absent from the LVS sample. The processed spectra for LVS (Figure 16), SchuS4 (Figure 17) and composite LVS/SchuS4 (Figure 18) are presented below.

The results from the TBAH derivatization largely confirmed the results from the acidic experiments; peak 2 appeared to be significantly larger in the virulent strain, while peak 4 appears to be significant enlarged in LVS. The processed spectra for LVS (Figure 19), SchuS4 (Figure 20) and composite LVS/SchuS4 (Figure 21) are presented below. Peaks A-D corresponds with the butyl esters of previously identified compounds. The TBAH difference maps (Figure 22 and Appendix B) also suggest that SchuS4 may be additionally enriching tetra and octadecenoic acid components of its lipid membrane. Upon further investigation, decanoic acid $\rightarrow$ tetradecanal is generally accepted to be a function of bacterial membrane plasticity generally associated with bacterial remodeling of the lipid membranes in response to temperature (as temperature decreases the bacterium installs shorter chained lipids to maintain membrane plasticity). ${ }^{107}$

\section{Ft Lipid Conclusions and Discussion}

The purpose of the original lipid workflow was to use nondestructive means to more accurately target more destructive analytical techniques. The single and multidimension NMR work illustrated the lipids of interest resided in a complex matrix of cellular and laboratory contamination. The use of semi-preparative LC allowed lipid fractions to be tested discretely; however, the absolute recovery suffered greatly as the 


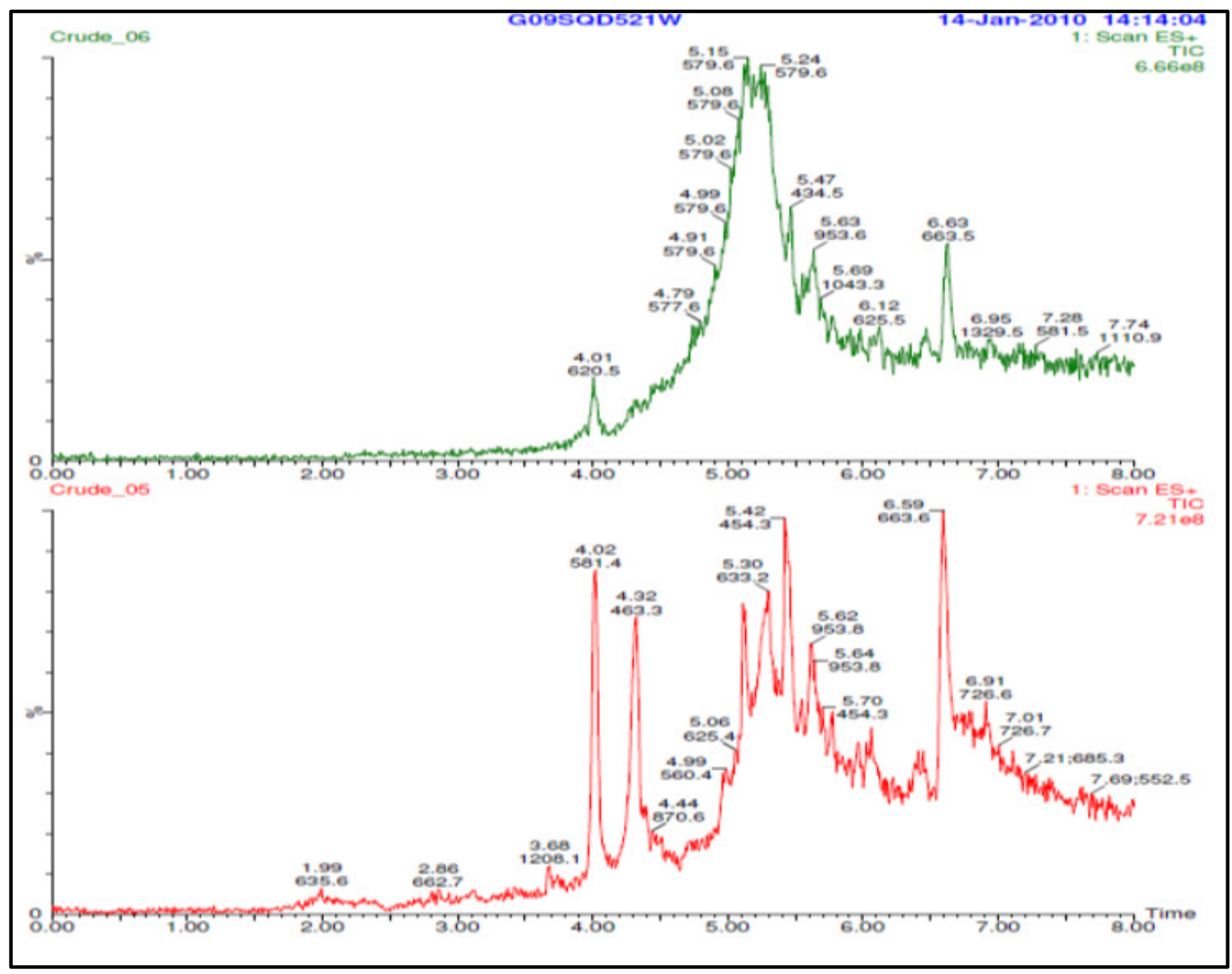

Figure 13 Serial Concentration UPLC/MS of Non-polar LVS (Top) and SchuS4 (Bottom) Fraction Pools

NOTE: The two significant SchuS4 peaks at $581.4 \mathrm{~m} / \mathrm{z}$ (Butylstyrene) and $463.3 \mathrm{~m} / \mathrm{z}$ (Polypropylene) were determined to be laboratory contaminates. 


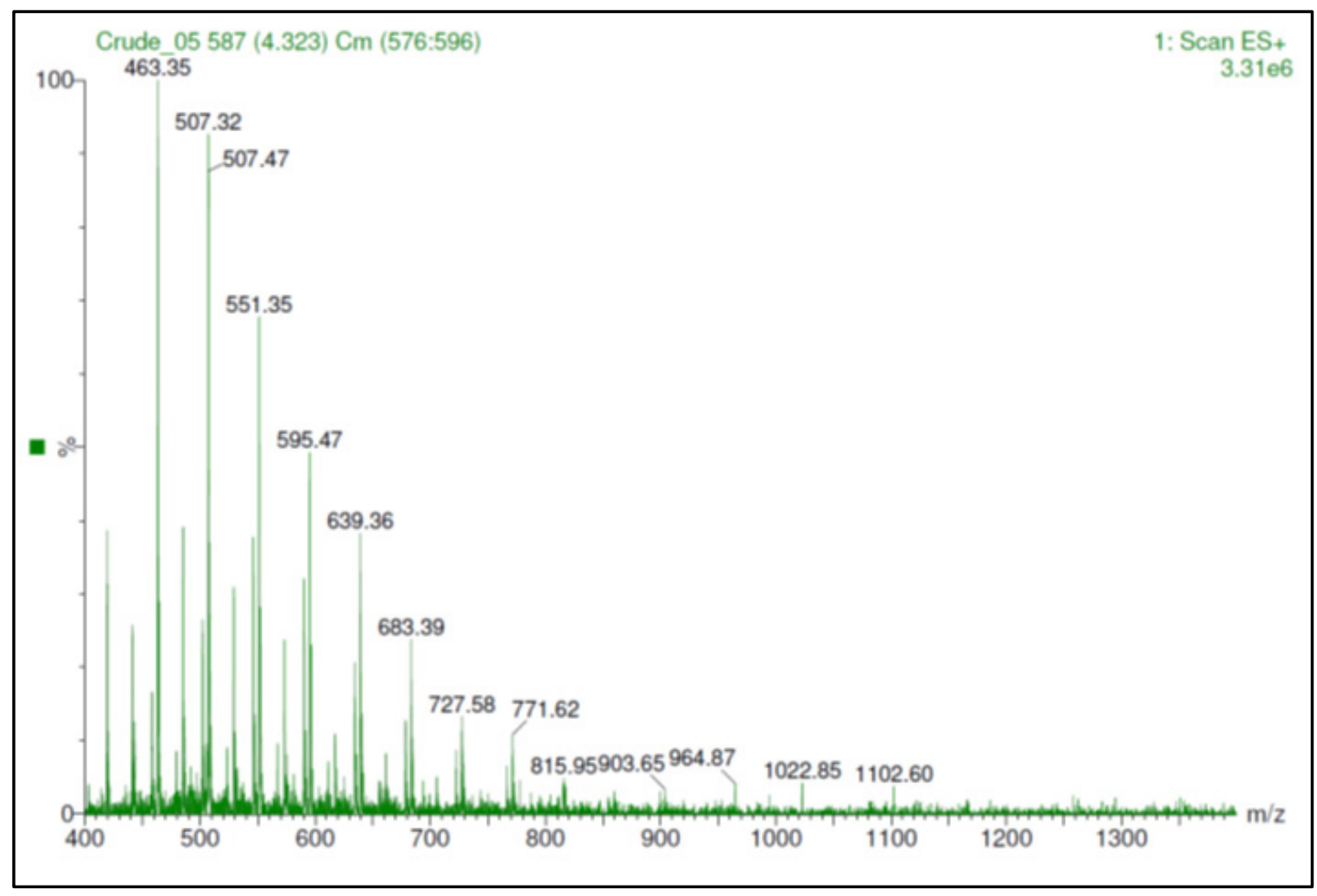

Figure 14. Extracted Spectra from $T_{R} 4.323(463.3 \mathrm{~m} / \mathrm{z}) \Delta 44 \mathrm{~m} / \mathrm{z}$ Indicative of Polypropylene Contamination 


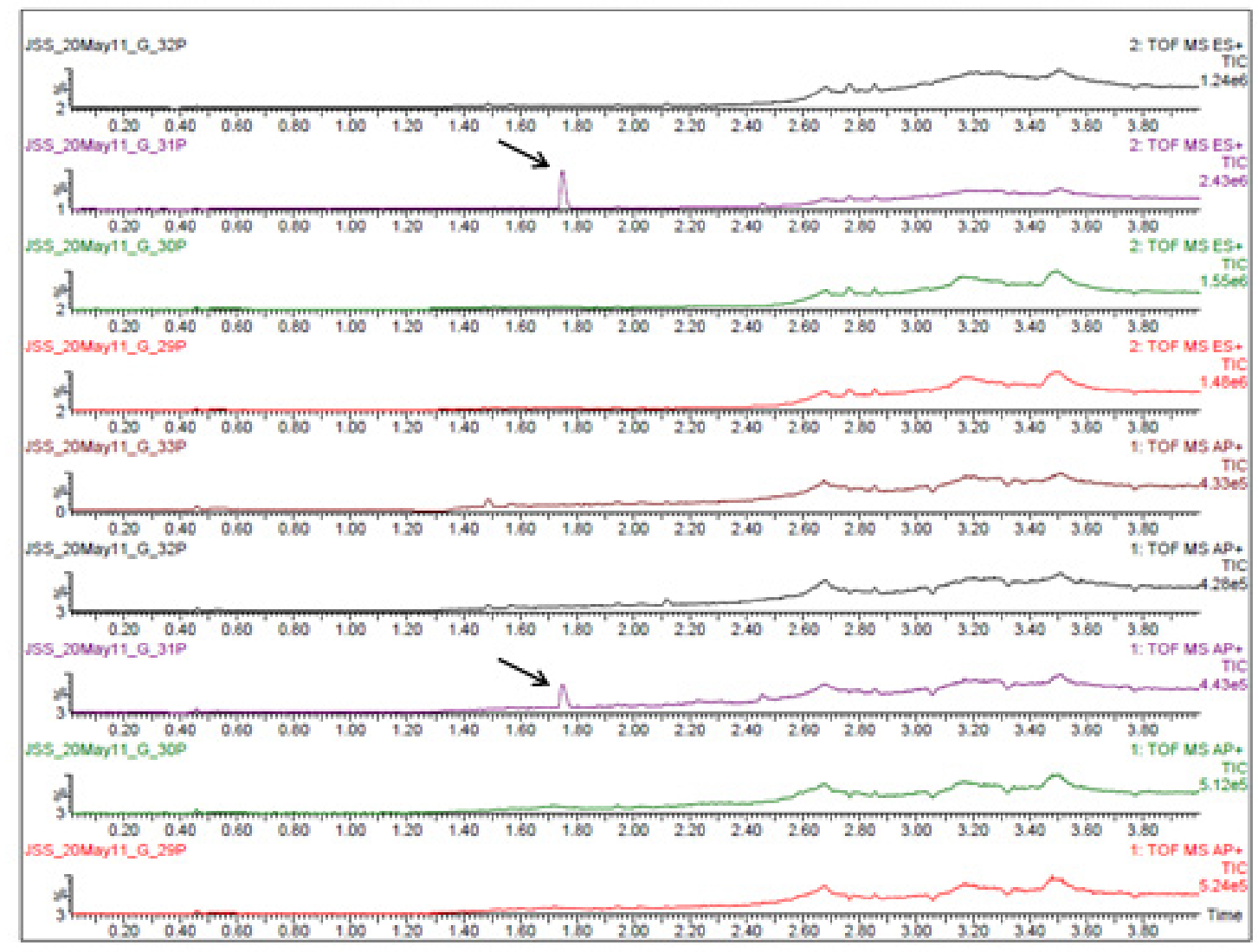

Figure 15. The Data Directed (MS ${ }^{e}$ ) TIC of SchuS4 Fractions 29-34 Displayed Similar Contamination 


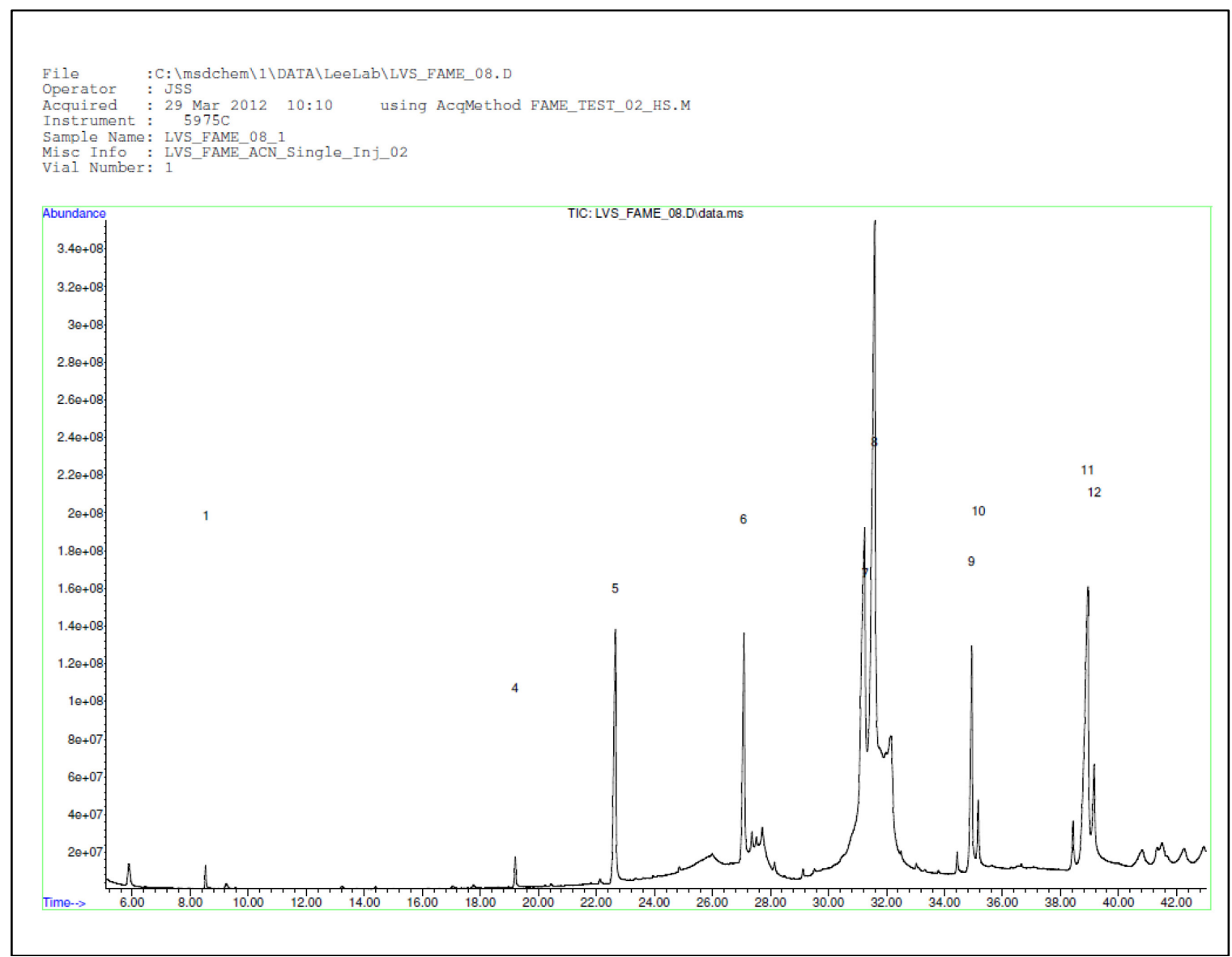

Figure 16. GC/MS Spectra of $\mathrm{HCl}-\mathrm{CH}_{3} \mathrm{OH}$ Fatty Acid Methyl Ester Transesterification Products of LVS Acetone Soluble Lipid Extract 


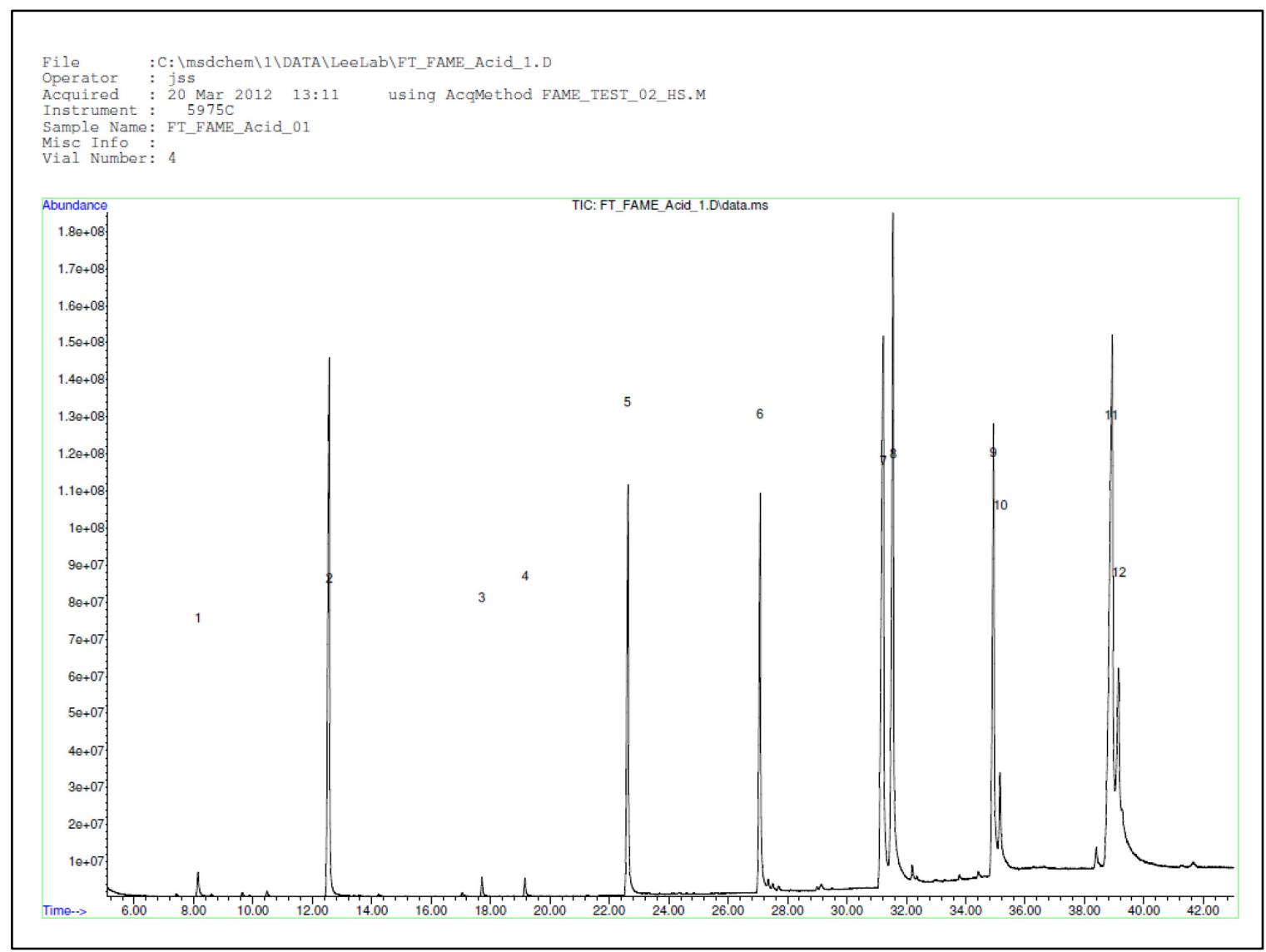

Figure 17. GC/MS Spectra of $\mathrm{HCl}-\mathrm{CH}_{3} \mathrm{OH}$ Fatty Acid Methyl Ester Transesterification Products of SchuS4 Acetone Soluble Lipid Extract

NOTE: Peak two is of particular interest. 


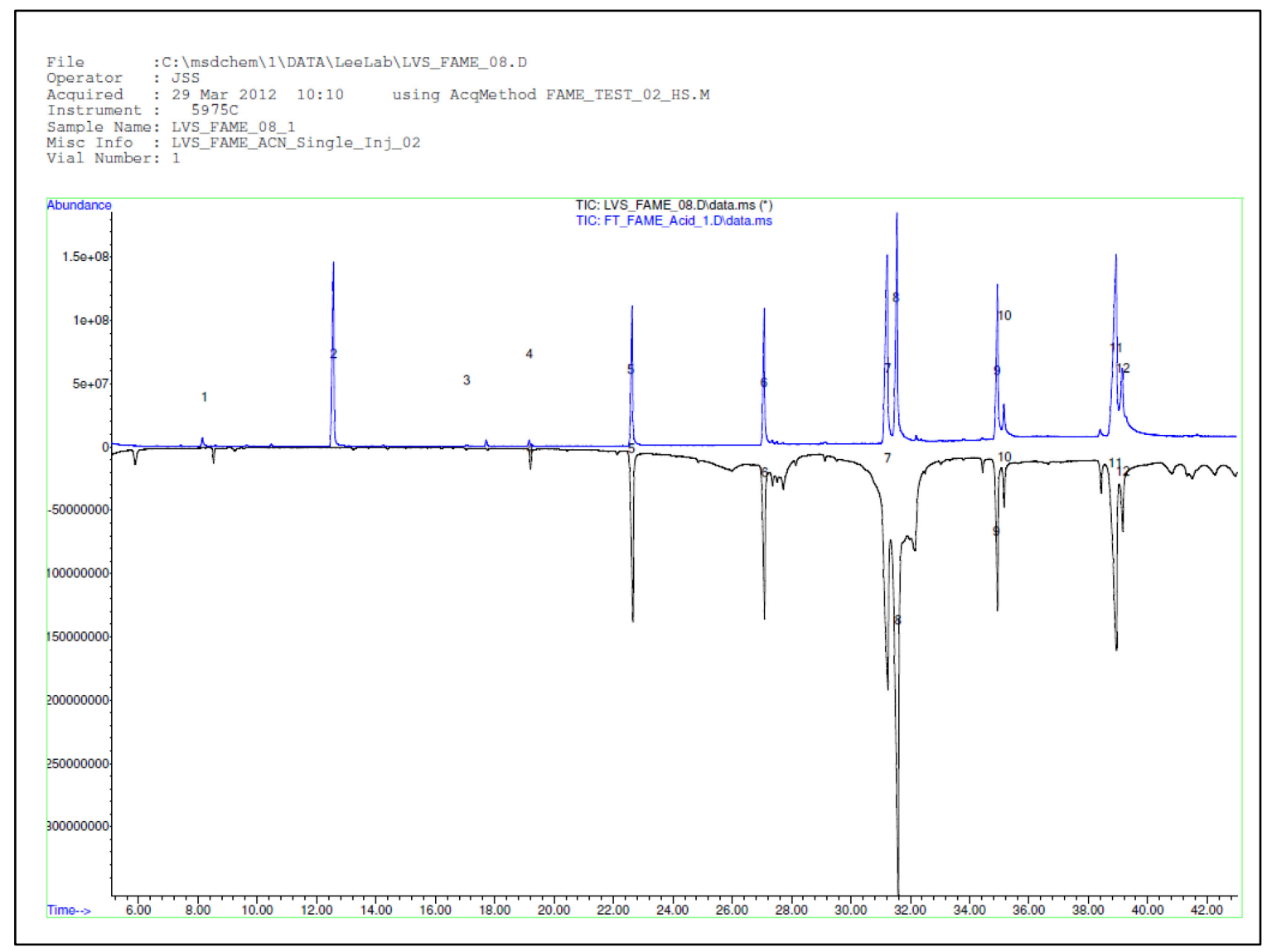

Figure 18. Composite of GC/MS (HCl-MeOH) Fatty Acid Methyl Ester Transesterification Products of SchuS4 (Positive) and LVS (Negative) Acetone Soluble Lipid Extracts 


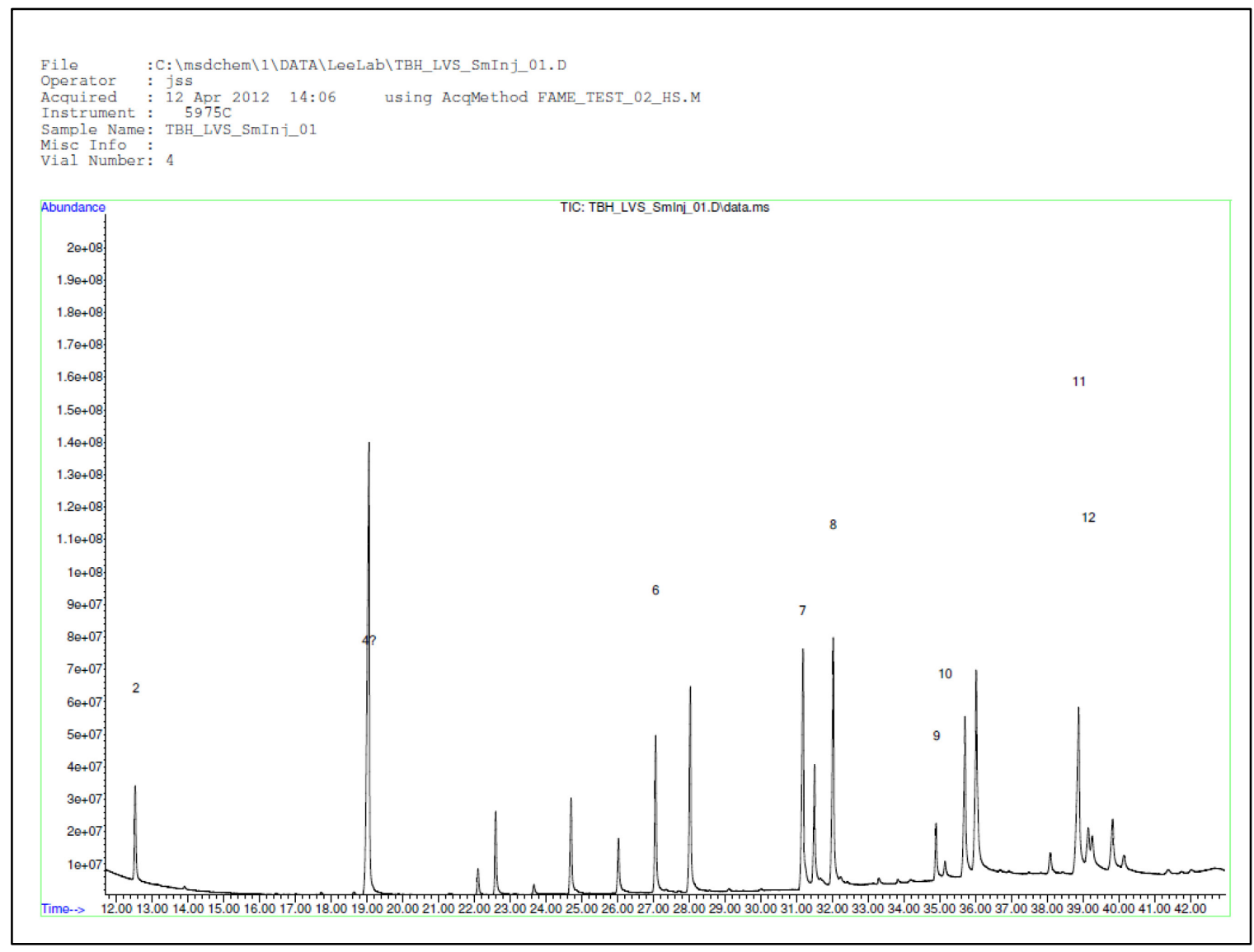

Figure 19. GC/MS Spectra of Base Catalyzed (TBAH) FAME Products of LVS Acetone Soluble Lipid Extract 


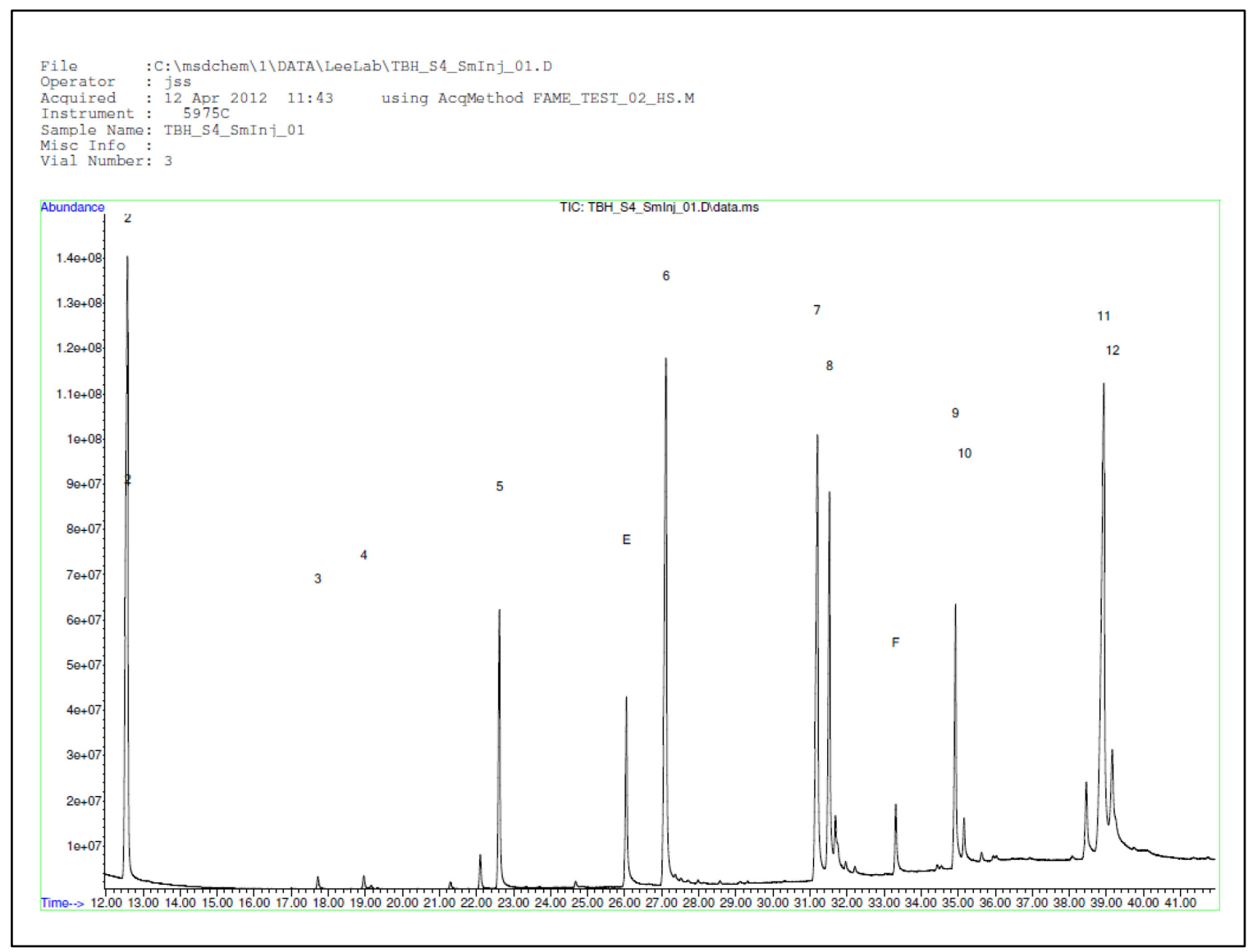

Figure 20. GC/MS Spectra of Base Catalyzed (TBAH) FAME Products of SchuS4 Acetone Soluble Lipid Extract 


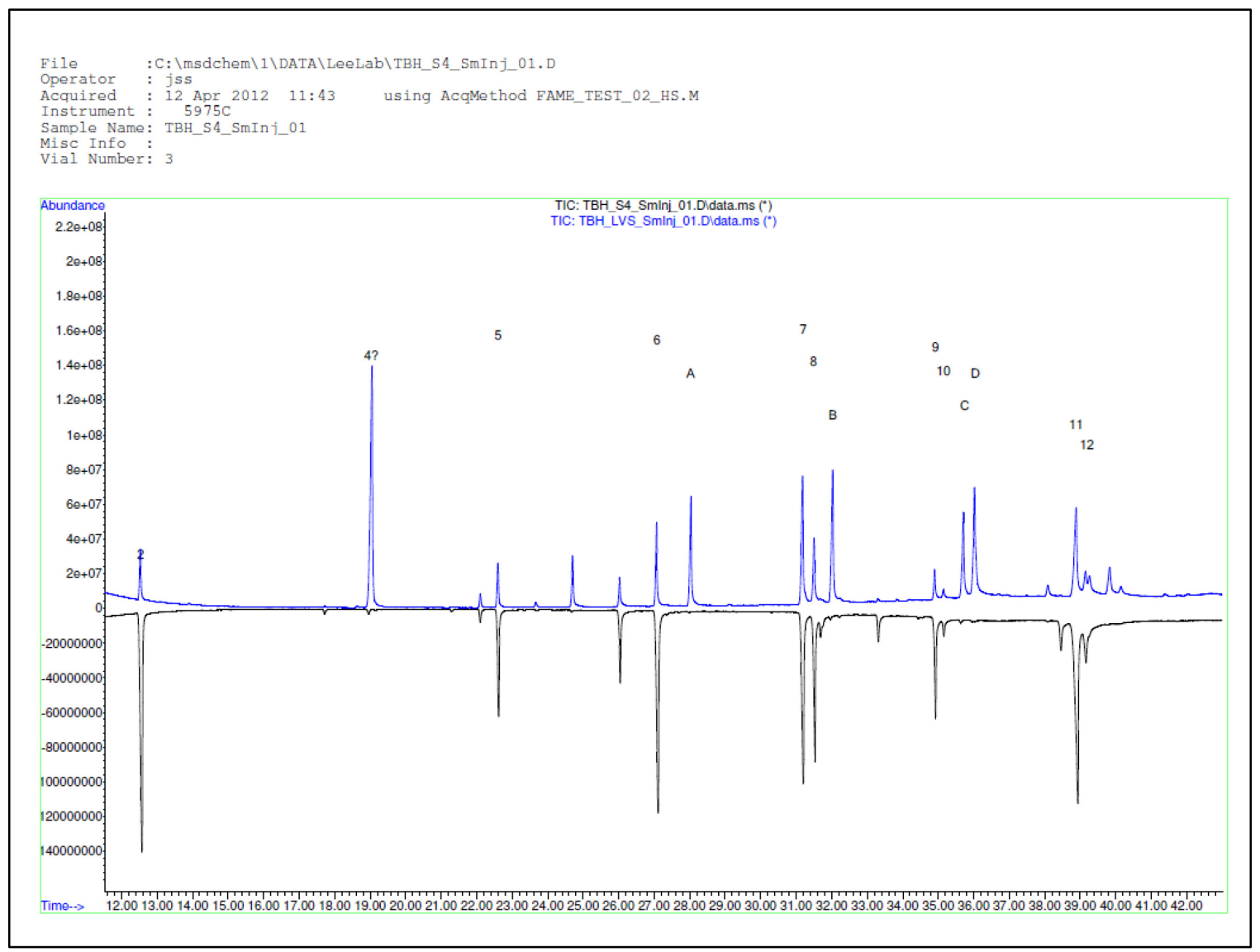

Figure 21. Composite of Base Catalyzed (TBAH) FAME Products of LVS (Positive) and SchuS4 (Negative) Acetone Soluble Lipid Extracts

NOTE: Difference maps were normalized for tetradecanoate. 


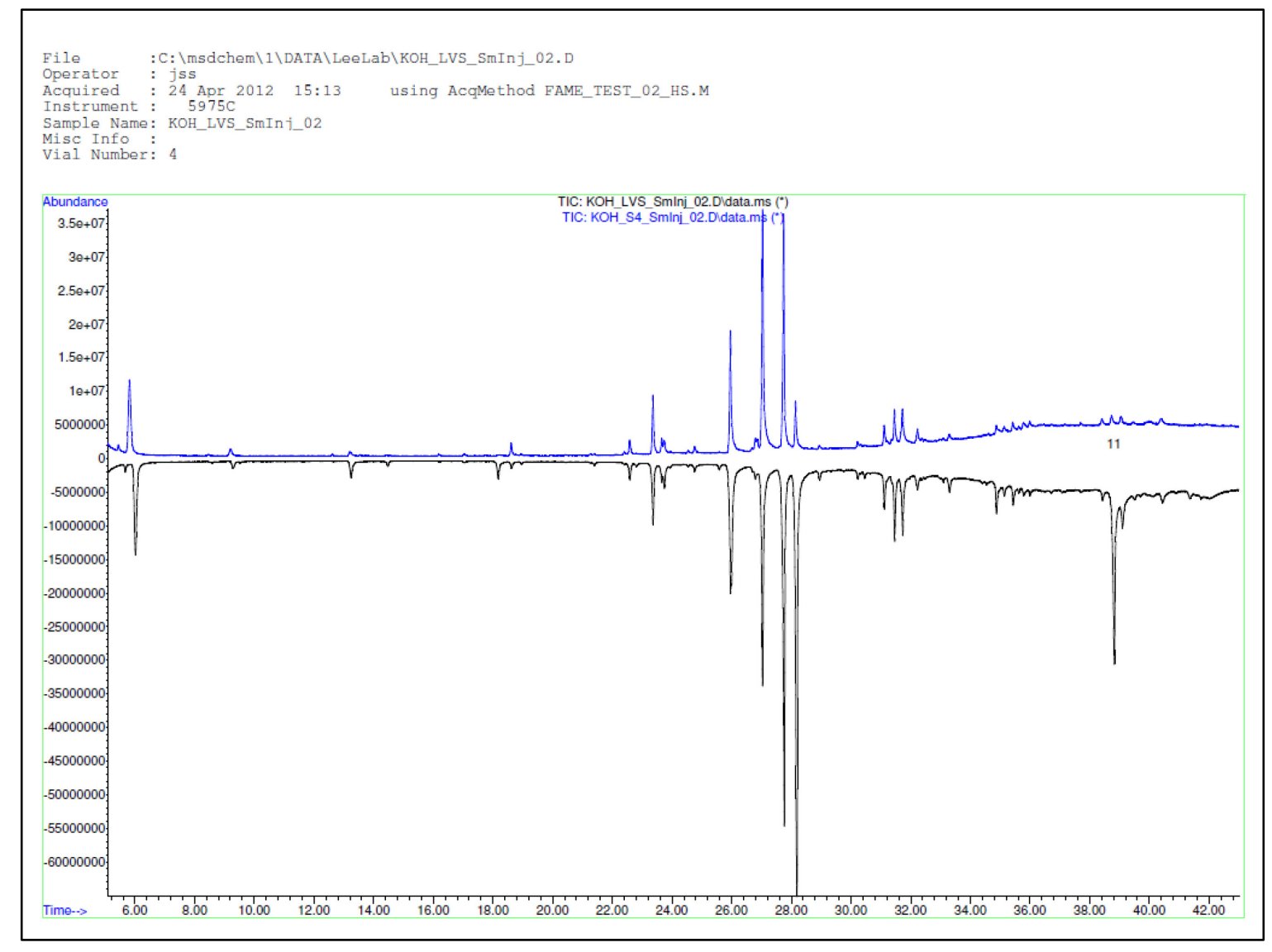

Figure 22. GC/MS (KOH) Fatty Acid Methyl Ester Transesterification Products of SchuS4 (Positive) and LVS (Negative) Acetone Soluble Lipid Extracts Normalized for Tetradecanoate

NOTES: In an attempt to futher validate SchuS4's proposed enrichment of peak two, a $\mathrm{KOH}$ supponification was attempted. Upon investigation, the only meaningful difference was in docosanoic acid (Peak 11)--further suggesting some divergences in the lipid profiles of the bacteria are likely resultant from differences in their environment (BSL2 vs BSL3). 
analytes are moderately soluble at best. The GC work served to illustrate a number of interesting results, SchuS4 expresses significantly more short chained (12:0/14:0) acids, while LVS appears to express longer chains (18:0). While a portion of this expression is likely a result of membrane homeostasis, it is interesting to note Lipid A surface expresses predominately (18:0) and Lipid A mutation has shown to play an important role in potentially protecting immune compromised mice. ${ }^{105}$

TLC/LTQ-MS experiments were designed and are currently underway to determine if the methyl esters identified via GC are part of a larger conjugated molecule (Lipid A) or if they exist as smaller free lipid species.

One of our primary goals in the design and implementation of the SchuS4 / LVS lipid analytical workflows was to leverage a number of nondestructive analytical techniques to target destructive, though informatically rich, mass spectrometric analyses. The preliminary NMR and TLC data provided considerable data including the presence of fatty acid components and common laboratory contaminates. In retrospect, as the LVS lipid components are expressed and purified in standard laboratory glassware (BSL1+) while the growth of virulent SchuS4 requires elevated precautionary measures (BSL3+) a few of which are the exclusion of glassware and use of plastic cultureware, a certain level of plastics and plasticizer contamination in the SchuS4 extracts was unavoidable.

Initially we sought to overcome this contamination through the use of semipreparatory flash chromatography. Three pools of SchuS4 fractions retained tractable activity upon separation, however the limited solubility if the lipid products greatly diminished recovery. We realized from the outset any chromatographic system would afford analytical losses. Our hope was the acquisition of highly meaningful correlational 2D NMR would prove the sacrifice worthwhile. Unfortunately the 2D spectra remained highly complex and inappropriate for correlational spectroscopy, as multiple peaks overlapped in key areas.

The GC transesterification proved highly successful to study lipid content as it required little analyte and the chemical derivatization and chromatographic separations removed many of the existing contaminates. The discovery of the short chain fatty acids (SCFA) found only in the SchuS4 extract is thought to be highly significant. The question now is to determine if these SCFA lipids exist on their own functioning as immunesuppressive agents or if they are conjugated into macromolecules for example Lipid A or if both may be true. The biological basis for either chemical theory appears conceivable as the limited genetic material available $(2.3 \mathrm{Mb})$ suggest $\mathrm{Ft}$ is genetically unable to afford many of the more advanced defense mechanisms via the synthesis of complex polyketides or non-ribosomal peptides as witnessed in larger pathogens such as $P$. aeruginosa and M. ulcerans.

Over its evolutionary history as a pathogen Ft has developed many tools to subvert host immune response. The question now is do host immune bodies fail to recognize the impending threat? Or are they somehow deactivated through a small molecule modulator? The surface expressed lipid A head of LPS is a primary determinant 
of antigenic response to gram negative bacteria. A recent publication noted Ft's ability to remodel Lipid A. ${ }^{105}$ If the Lipid A antigen were adapted to express SC fatty acids as opposed to the more usual 18:0 and 20:0 systems it is interesting to hypothesis that the host immune bodies via innate Toll signaling pathway may not be activated by virulent strains of $\mathrm{Ft}$, at least for a time.

To this end a TLC/Direct Injection LTQ-MS experiment was developed in an attempt to conclusively identify the SCFA conjugation products of Ft SchuS4. While we only have preliminary data at this time, the initial results appear to confirm the experimental design. The phosphatidylethanolamine (Figure 23) head group contains seven carbons, the spectra indicates 32 to 36 total carbon (Figure 24), as there are generally symmetric-2 lipid prosthetics the lipid moieties appear to range from 10 to 14 carbons in length supporting results from our GC-MS analysis. We have also identified phosphotidylcholine (Figure 25) though it displays a more usual lipid chain length. ${ }^{108}$

\section{Development of a Generic Bacterial Natural Product Analytical Workflow with Applications from the Analysis of Mycolactone}

The analysis of bacterial natural products (BNPs) presents a number of somewhat unique challenges to the laboratory chemist, many due to the amazing diversity inherent to NP libraries. While no consolidated BNP evaluation workflow currently exists, there are a number of proven analytical techniques directly compatible to work in BNP isolation and classification. An unfortunate caveat exists throughout many of these analyses, however, in that a pure analytical sample is predominantly required to generate meaningful data. To this end, many BNP workflows are subdivided into two primary components; purification and analysis.

The primary focus of our purification technique is to separate the unknown complex fraction into multiple, reasonably pure fractions, while recovering as much of the initial analyte as possible. Conceptually, this technique may be applied in one of two ways; the bulk fractionation of a complex mixture (as is often the case when working with large quantities of crude material or in industrial applications) or an analytically driven "targeted fractionation" approach. Given the relatively limited quantity of analyte, a target driven fractionation approach appears to be the most prudent.

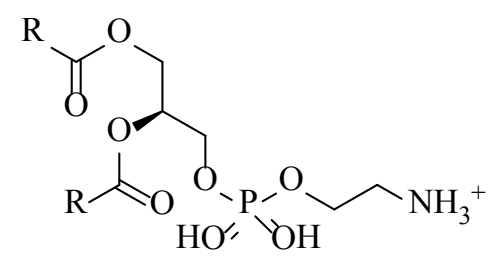

Figure 23. Structure of Phosphatidylethanolamine Head Group 


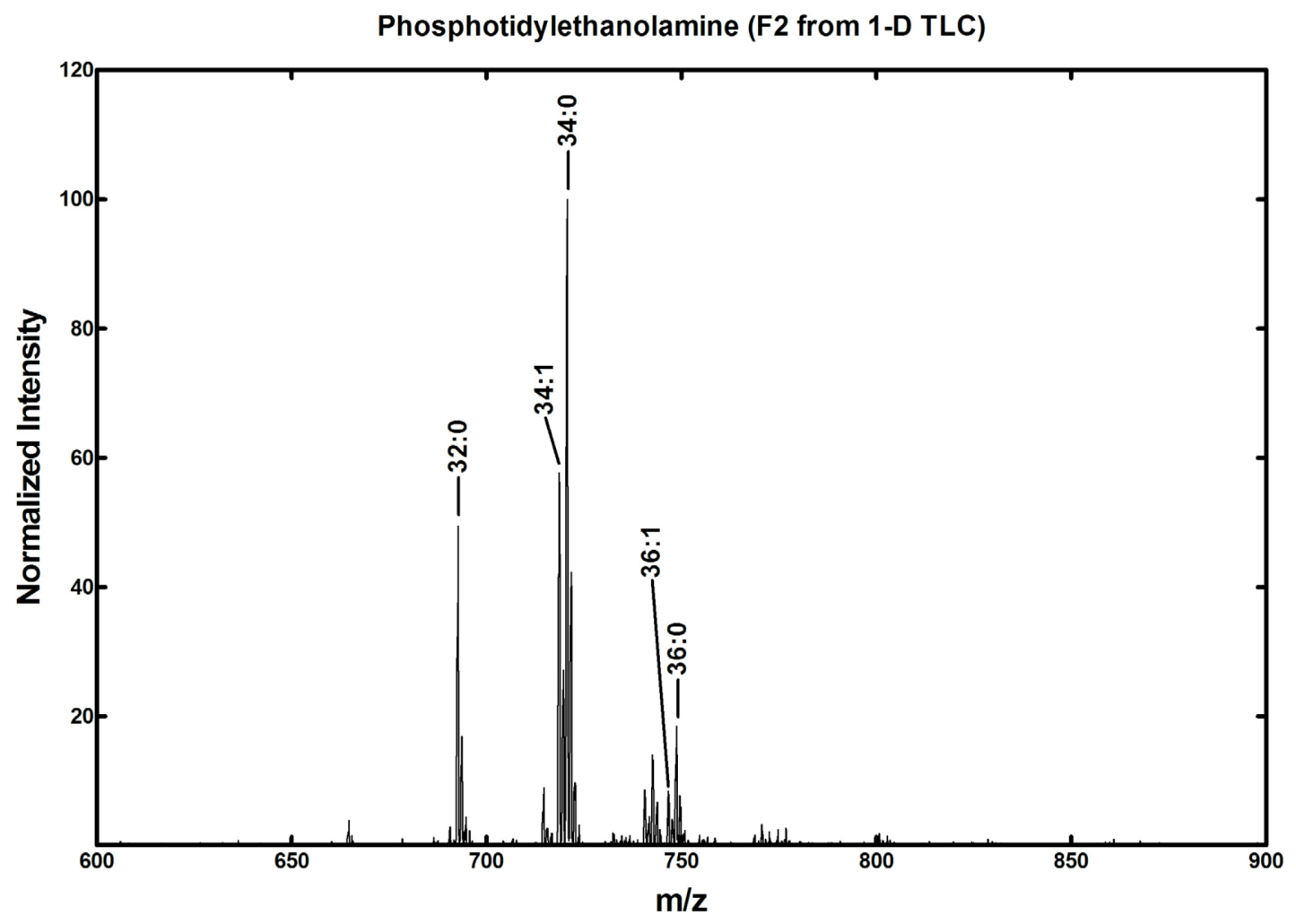

Figure 24. LTQ-MS of Phosphatidylethanolamine 


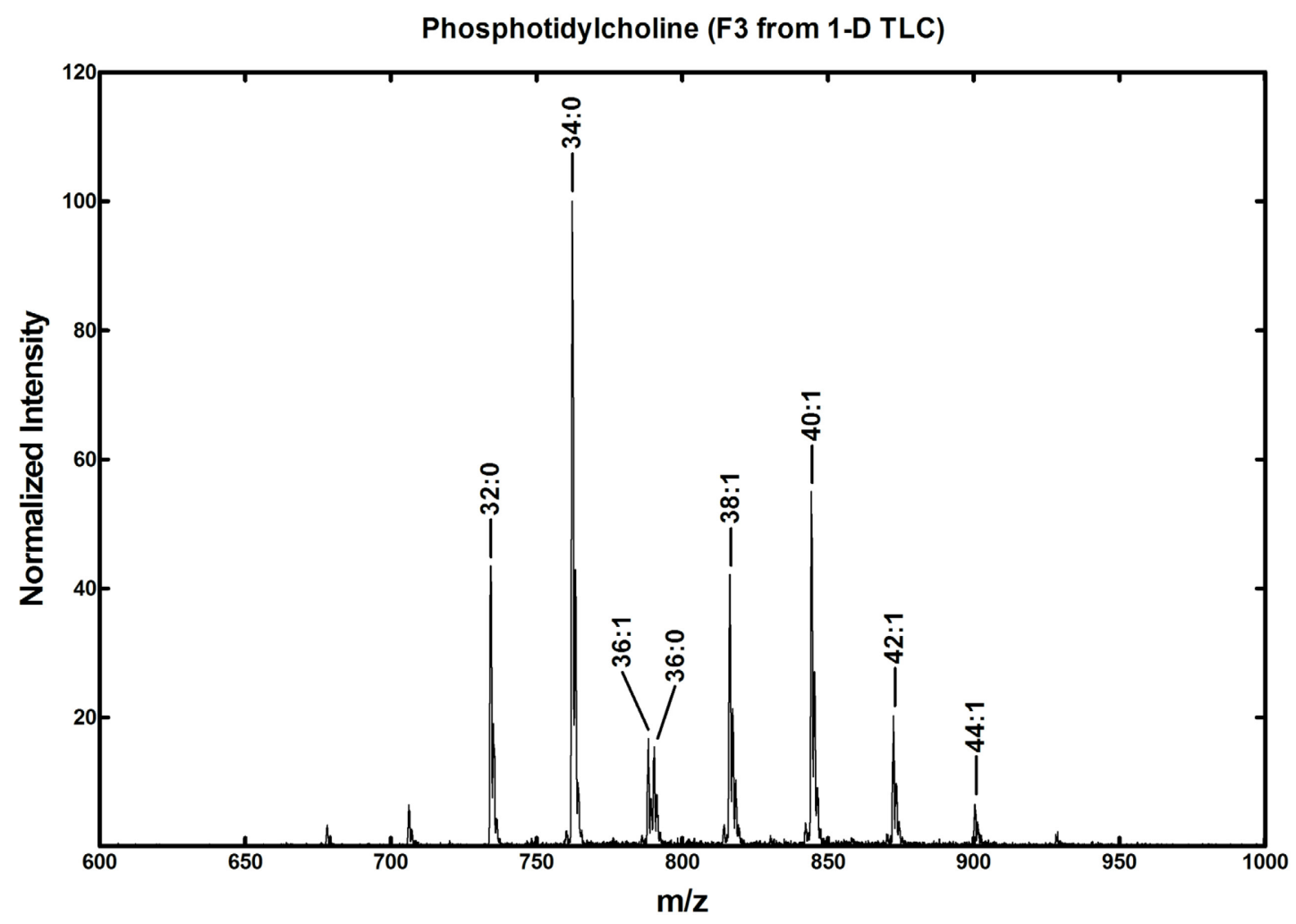

Figure 25. LTQ-MS of Phosphotidylcholine 
The primary focus of our purification technique is to separate the unknown complex fraction into multiple, reasonably pure fractions, while recovering as much of the initial analyte as possible. Conceptually, this technique may be applied in one of two ways; the bulk fractionation of a complex mixture (as is often the case when working with large quantities of crude material or in industrial applications) or an analytically driven "targeted fractionation" approach. Given the relatively limited quantity of analyte, a target driven fractionation approach appears to be the most prudent.

As the fraction of interest appears to contain a chemical species affording high activity against a particular bioassay, a bioactivity driven secondary fractionation may offer the best odds of identifying the active compound, also as the identity of the plant is known, a literature search may be useful in identifying previously isolated active components. Over the past two years, our lab has taken interest in the bioactivity driven fractionation of naturally derived compounds, if a selective bioassay exists, they often afford levels of detection far superior to traditional analytically driven techniques.

Some limited MS data can be greatly helpful in preparing for a secondary fractionation run. A small aliquot of material sampled on the HRMS in both ESI(+) and ESI(-) full scanning mode offers insight into compound polarity (this aided by initial fractionation conditions and elution time) apparent ionization efficiency and fraction complexity. In a somewhat method and analyte dependent fashion, the $\mathrm{pKa}$ of a compound may be estimated by comparing its elution time against a series of known compounds, in much the same way as gel based systems use molecular weight "ladders" to qualitatively identify the molecular mass of an unknown protein. While single point data may provide useful, these estimations become more robust with the inclusion of orthogonally referenced elutions. Aided by these estimations, the empirically measured high resolution mass data can be used to predict prospective chemical formula for the NPs while the UV detector offers secondary detection of compounds containing accessible UV chromophores.

The secondary fraction of a BNP is conceptually quite simple but often difficult to achieve in a laboratory setting. The hope is to separate the BNPs using a method more or less orthogonal to the original (Standard Phase $\mathrm{CHCl}_{3}: \mathrm{CH}_{3} \mathrm{OH}$ ) fractionation conditions. We have evaluated a number of semi-analytical chromatographic conditions for their ability to provide selectivity contrasting with $\mathrm{CHCl}_{3}: \mathrm{CH}_{3} \mathrm{OH}$ separations. While HILIC is often referenced as a hybrid of conventional and reverse phase chromatographic techniques (for its reversal of conventional RP elution orders) we have been unable to achieve reasonable recoveries with this technology.

In our laboratory we have relied on Phenomenex pentafluorophenyl (PFP) stationary phase chemistries to provide a somewhat contrasting separation technique compared to conventional C18 (PFP is a reverse phase column and uses mobile phases similar to $\mathrm{C} 18$ but is specially hybridized by the inclusion of a polar-cyclic pentafluorophenyl motif that differentially retains many of the aromatic and polar compounds often encountered in natural products). While we have found success using this type of secondary fractionation, especially when the samples are not overly complex, 
I feel the investigation of a truly orthogonal separation technique is warranted and will increase the robustness of any subsequent BNP publications. To this end, I am currently investigating semi-analytical SFC based techniques for their potential application to our BNP workflow.

Both techniques offer potentially complimentary selectivity, however, I have found many of my standard phase BNP separations often offer low recoveries when analytes are in the milligram range, we have recently acquired low volume (4 gram) deactivated silica columns for use with our isolera flash chromatography systems which should aid in sample recovery. We are currently evaluating a BNP workflow in which the primary preparative fractionation relies on standard phase ELSD coupled deactivated silica "flash" chromatography while subsequent semi-preparative steps utilize traditional C18 based separations; thus far, this methodology appears to provide a truly nonequivalent, complimentary separation technique.

In similar fashion, I believe a SFC based semi-preparative approach may be most orthogonal to the $\mathrm{C} 18$ based primary separations. This might also prove to be advantageous over our standard phase techniques, as SFC potentially offers both increased recoveries and avoids the difficulties associated with the handling and desolvation of $\mathrm{CHCl}_{3}$. From this point in the analytical workflow HRMS data coupled with one of the previous semi-preparative techniques should prove sufficient to separate the initial complex hit into a number of relatively pure fractions. These high purity fraction(s) should then be re-submitted to the bioactive assay to locate the fraction(s) responsible for the initial hit. Once high purity fraction(s) are recovered, a number of analytical techniques become available for the evaluation of the active chemical compound(s).

The analysis of BNP components often relies on two very different analytical approaches, NMR and MS. Many labs, including our own, often submit chemical hits to a somewhat standardized NMR analysis prior to conducting any MS work. NMR based BNP analytical techniques greatly benefit from the nondestructive nature of the NMR, coupled with the fact any materials transferred to dueterated solvents may be easily analyzed via MS. While NMR experiments are more contingent upon the recovery of a sufficient sample (1-2 mg for proton, $2-5 \mathrm{mg}$ for conventional ${ }^{13} \mathrm{C}, 10 \mathrm{mg}$ or more for correlational studies), they offer a potential array of chemical information from simply the number of carbons and hydrogens contained by a compound to detailed structural information from spin correlational or NOE type experiments through a nondestructive means.

Once the NMR work has been completed the MS work may begin, as mass spectrometry is inherently a destructive technique, care must be taken in the sequencing of the MS analyses. I often acquire another HRMS ESI(+) and ESI(-) full scan (Figure 26) of the active re-fractionated samples to provide a point of reference for future analyses, as before, prospective chemical formula(s) may be generated from the high resolution isotopic and mass defect measurements. From this point, the analytical workflow may fork into two possible directions, one includes operating the HRMS in a 


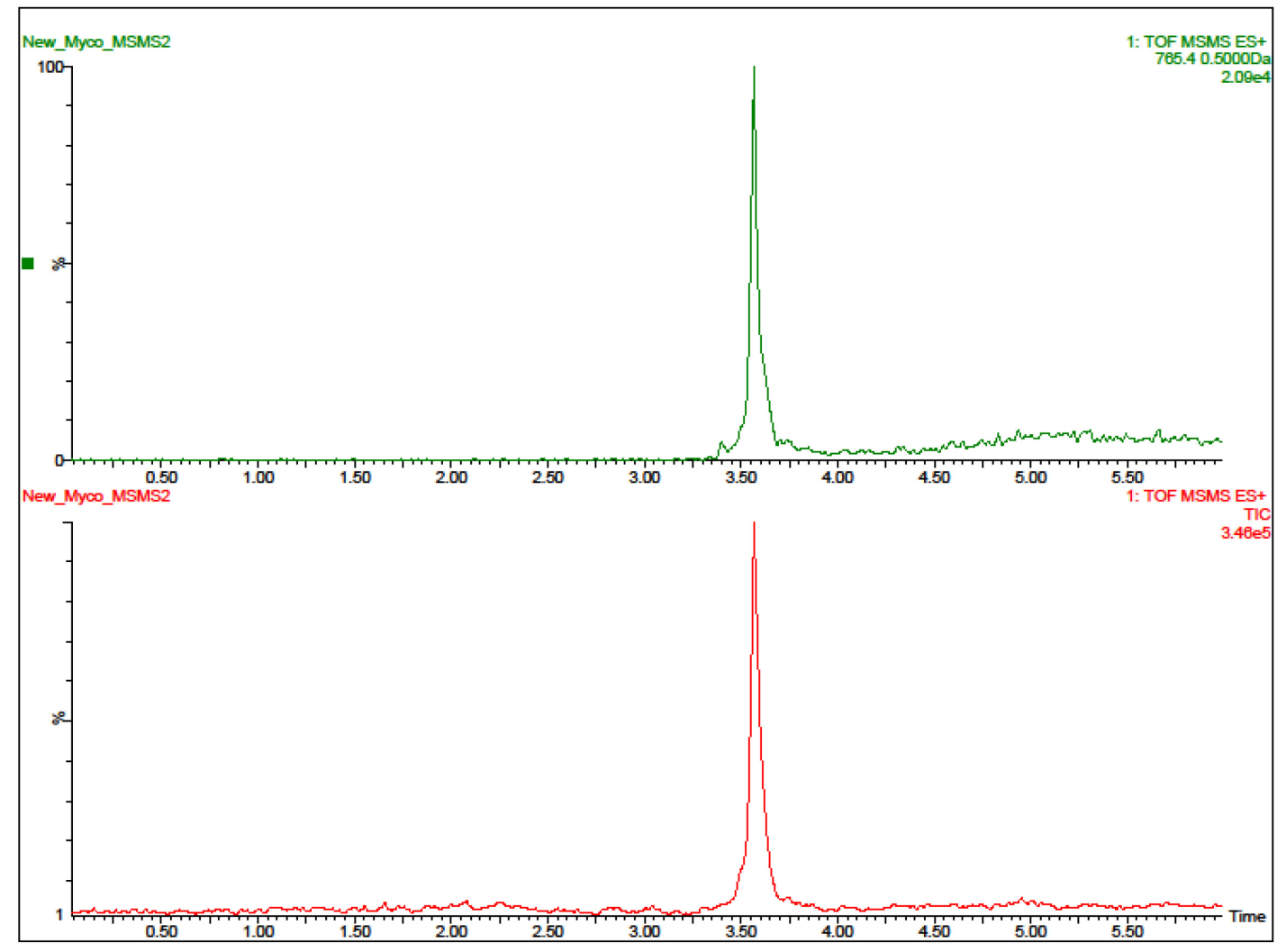

Figure 26. UPLC Targeted TQD-MS/MS of Control Mycolactone $(765.4 \mathrm{~m} / \mathrm{z})$ Served to Validate MS/MS Quantification Techniques 
way as to effectively overvolt the sample cone (octopole) thereby inducing nonspecific electronic fragmentation (in this way essentially mimicking the collision cell on the TQD), the second relies on the use of the TQD to induce kinetic fragmentation at/in its Q2 collisional cell. (Figure 27 illustrates the kinetic induced fragmentation of the parent mycolactone, $765 \mathrm{~m} / \mathrm{z}$, to the mycolactone core, $429 \mathrm{~m} / \mathrm{z}$, before (A) and after (B) exposure to sunlight.) (Structure of mycolactone provided in Figure 28) Electronic and kinetic fragmentation experiments are often complimentary in the profiles generated. The HRMS induced fragmentation experiments may be conducted while running the previously discussed full scans, in this way, a great deal of information may be gleaned from a limited amount of analyte.

Traditionally the neutral losses (NL) in an unknown analytes kinetic fragmentation spectrum have been used to provide insights into a molecule's fragmentation motifs and its ultimate functionalization. In the case of the TQD, these experiments may be conducted through the utilization of NL scans or through the creation of MRM experiments. These single or multiple reaction monitoring (SRM,MRM) processes rely on their ability to preselect one or multiple parent molecule(s) at the first quadrupole (Q1), subject this parent molecule to a kinetic fragmentation event (Q2) and then analyze the resultant fragments through the use of a final scanning quadrupole (Q3). (In a careful experiment, the kinetic potential of Q2 can be tuned to allow for the enrichment of the mycolactone core while providing sufficient molecular ions to discriminate between mycolactone $(765 \mathrm{~m} / \mathrm{z})$ and tetrahydroxymycolactone $(747 \mathrm{~m} / \mathrm{z})$. The net effective fragmentation event can then be ramped to provide fragments in an ascending order from the most electronically accessible functional groups to the more shielded molecular scaffold.

In this way the TQD's capability to precisely control the fragmentation process provides the analytical chemist a powerful tool in the ability to back-calculate many pieces of a molecule, as noted, the HRMS may be used to provide a similar but much less precise fragmentation process. This serves to illustrate a theme expressed throughout NP work; data rich MS experiments are often sample rich as well, the HRMS fragmentations are useful as they provide much of the TQD NL/MRM data while only consuming a fraction of the sample necessary to construct a conventional MRM experiment.

\section{Introduction to Mycobacteria ulcerans}

The causative agent of Buruli ulcer, Mycobacterium ulcerans $(\mathrm{Mu})$, is the third most common form of mycobacterial infection in humans after only TB and leprosy. ${ }^{109}$ The early stages of ulcerans infection often manifests as a small, dermal papule. Without proper diagnosis and treatment, the bacterium slowly invades and necrotizes the surrounding tissues through the secretion of the toxin mycolactone. ${ }^{110}$ (Figure 28). As the affliction of Buruli ulcer is primarily concentrated in developing nations located in tropical areas of the world ${ }^{111}$ a number of laboratories, ours included, have been investigating the possibility of a low cost means by which to remediate the progression of ulcerans infection. 


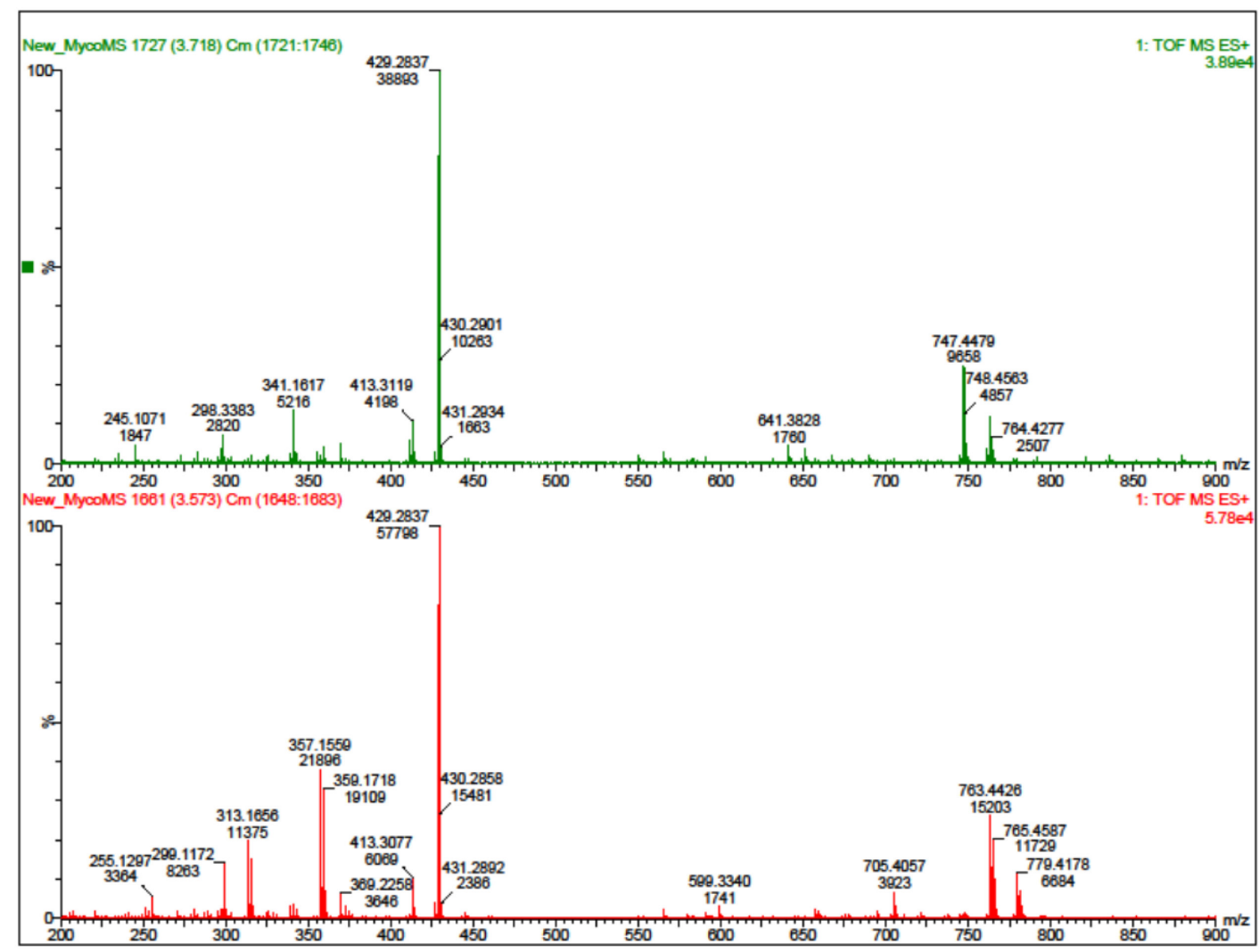

Figure 27. Targeted UPLC-TQD-MS/MS of Mycolactone Subspecies $747.4 \mathrm{~m} / \mathrm{z}$ (Top) and $765.5 \mathrm{~m} / \mathrm{z}$ (Bottom)

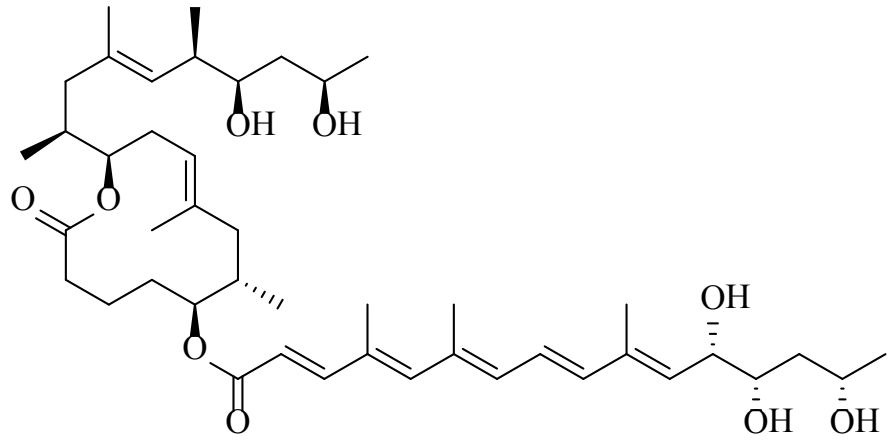

Figure 28. Structure of Mycolactone $(\mathrm{m} / \mathrm{z}=765)$ 
Several laboratories (including our own) have noted the instability of isolated mycolactone. ${ }^{112}$ In collaboration with the Britton laboratory we further investigated the effects of natural and UV light on mycolactone stability and sought to evaluate the potential clinical relevance of such findings as a low cost approach to remediate the effects of mycolactone toxification. While our collaborators ${ }^{113}$ were primarily concerned with the in-vivo and in-vitro modeling of mycolactone photoablation, our laboratory aims were to isolate sufficient quantities of mycolactone and study the effects of UV and visible photo irradiation on the chemical composition of the toxin. The impure $M$. ulcerans lipid extracts were separated and analyzed for purity following a (now standardized) BNP workflow. Secondly, the photostability of mycolactone was assessed via mass spectrometric tools.

In a proof of concept experiment, methanolic solutions of our internally produced pure mycolactone were applied to transparent plates and slowly rotated until a thin film developed. The plates were then randomly divided into two groups, light and dark. The light group would be exposed for 30 to 120 minutes of "Memphis Sun" (atop the hospital parking deck at 1:00 PM on cloudless days throughout May and June with an outside temperature ranging between $78^{\circ}$ and $89^{\circ} \mathrm{F}$, controls were allowed to remain outdoors covered in ablative foil during the exposure in an attempt to differentiate thermal and photo-induced degradation processes). The thin films would be resolvated in $\mathrm{CH}_{3} \mathrm{OH}$ and divided for bioanalysis and MS (Figure 29) illustrates the conservation of $765 \mathrm{~m} / \mathrm{z}$ to 429 $\mathrm{m} / \mathrm{z}$ (indicative of mycolactone) prior to exposure to sunlight (A), the daughter peak was lost post-exposure (B) indicating the disruption of the polyene system. The bioanalytical technique involved treating human keratinocytes with aliquots of the light (E) and dark (F) mycolactone samples, while kill curves remained similar; the light group required a half-log increase in dose to achieve similar toxicity (Figure 30). In a comparison of mycolactone toxicity between batches (Figure 31) the new mycolactone stocks demonstrate significantly increased toxicity, illustrating the inherent instability of mycolactone thin film archival. While the structure of mycolactone was previously known, an effort was made to assay the analytical invasivity of our techniques due to the light sensitive nature of the compound. There was initial concern the UV/Vis detector might be capable of degrading the mycolactone under analysis (Figure 32), this proved to not be a concern as mycolactone parents $755 \mathrm{~m} / \mathrm{z}$ and $747 \mathrm{~m} / \mathrm{z}$ were unaffected, as no significant change in peak area was observed.

\section{Mycolactone Conclusions and Discussion}

The recent publication by Marion ${ }^{112}$ in many ways supports the findings of this work as well as suggesting a number of potential modifications to our current mycolactone production and storage strategies. We have successfully shown mycolactone's toxic potential is mediated through the exposure to sunlight. We were able to determine indoor light was harmful, though not nearly to the extent at sunlight (as confirmed my Marion). We determined conclusively UV/Vis flow spectrometry does not significantly damage mycolactone, likely due to the shielding effects of the effluent. We were also able to develop robust TQD based assays for both quantification and 


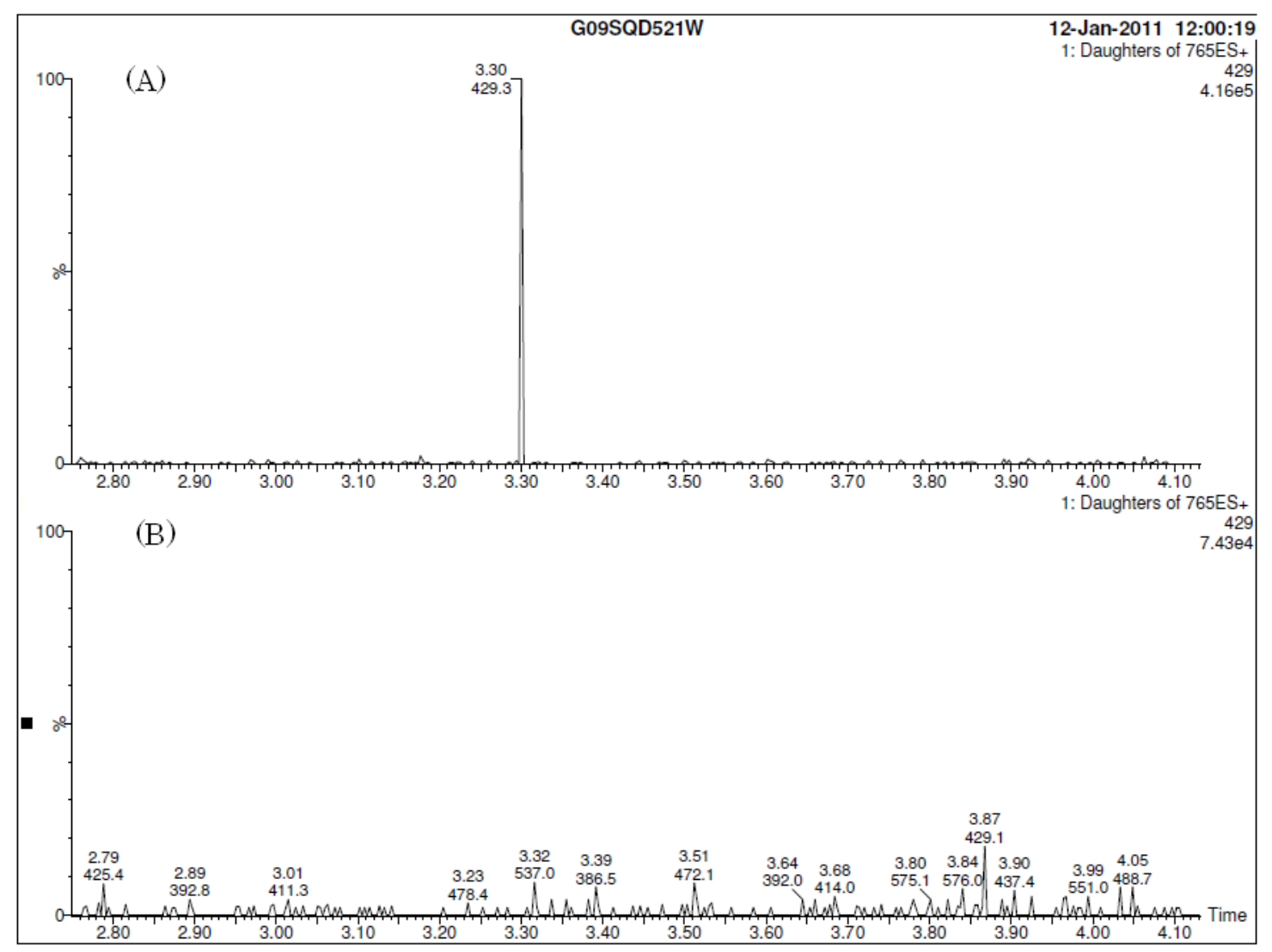

Figure 29. $765 \mathrm{~m} / \mathrm{z}$ Daughter Scan Indicating the Presence of the Mycolactone Core (429 m/z) Prior to Sunlight Exposure (A) and Post Exposure (B)

NOTE: Post exposure, the parent/daughter transition indicative of mycolactone could not be detected. 


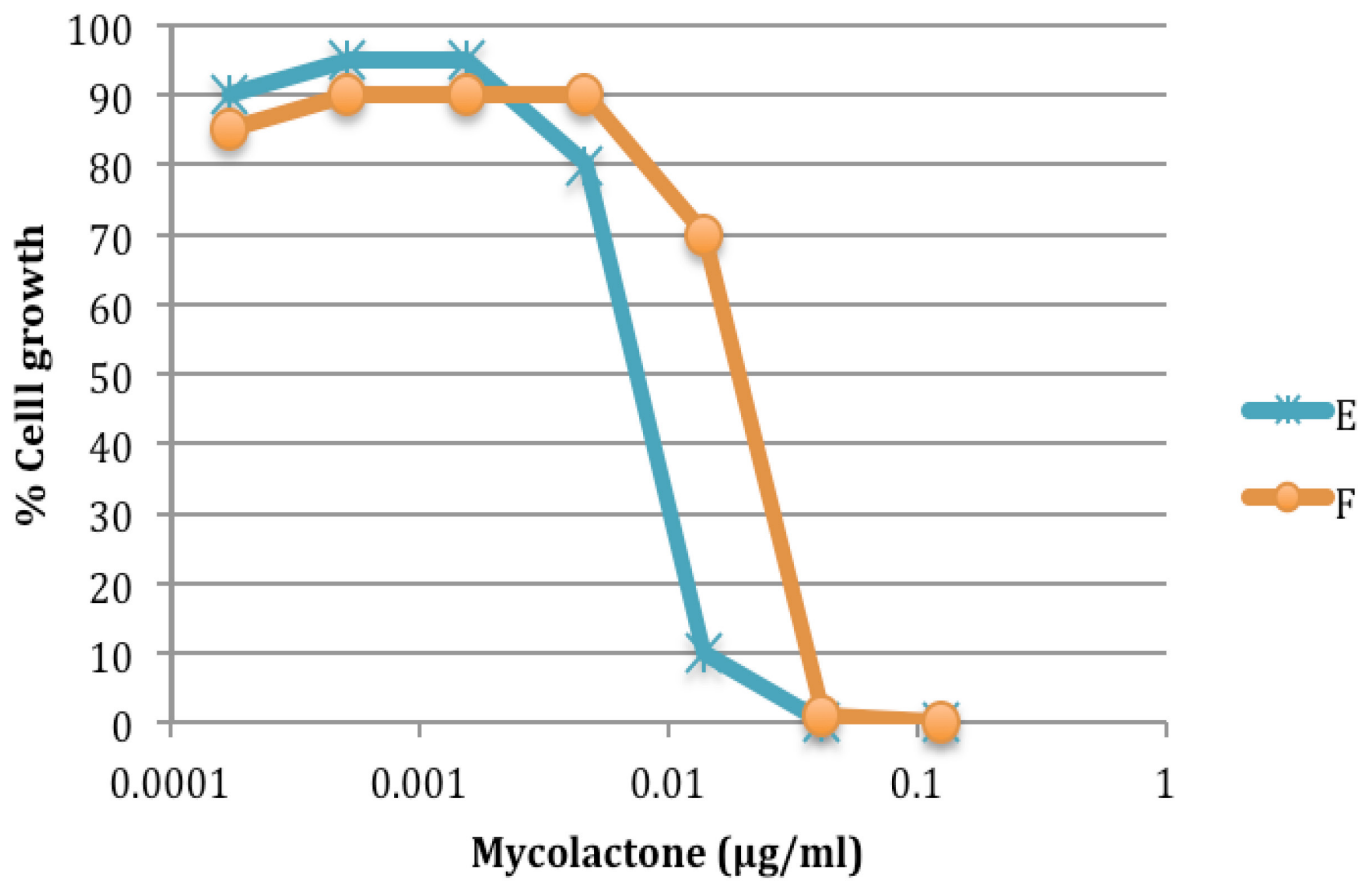

Figure 30. The Effect of Mycolactone Photoablation on Human Keratinocytes

NOTE: The UV exposed mycolactone stock $(F)$ suffers a $0.5 \log$ reduction in toxicity as compared to the control mycolactone $(\mathrm{E})$. 


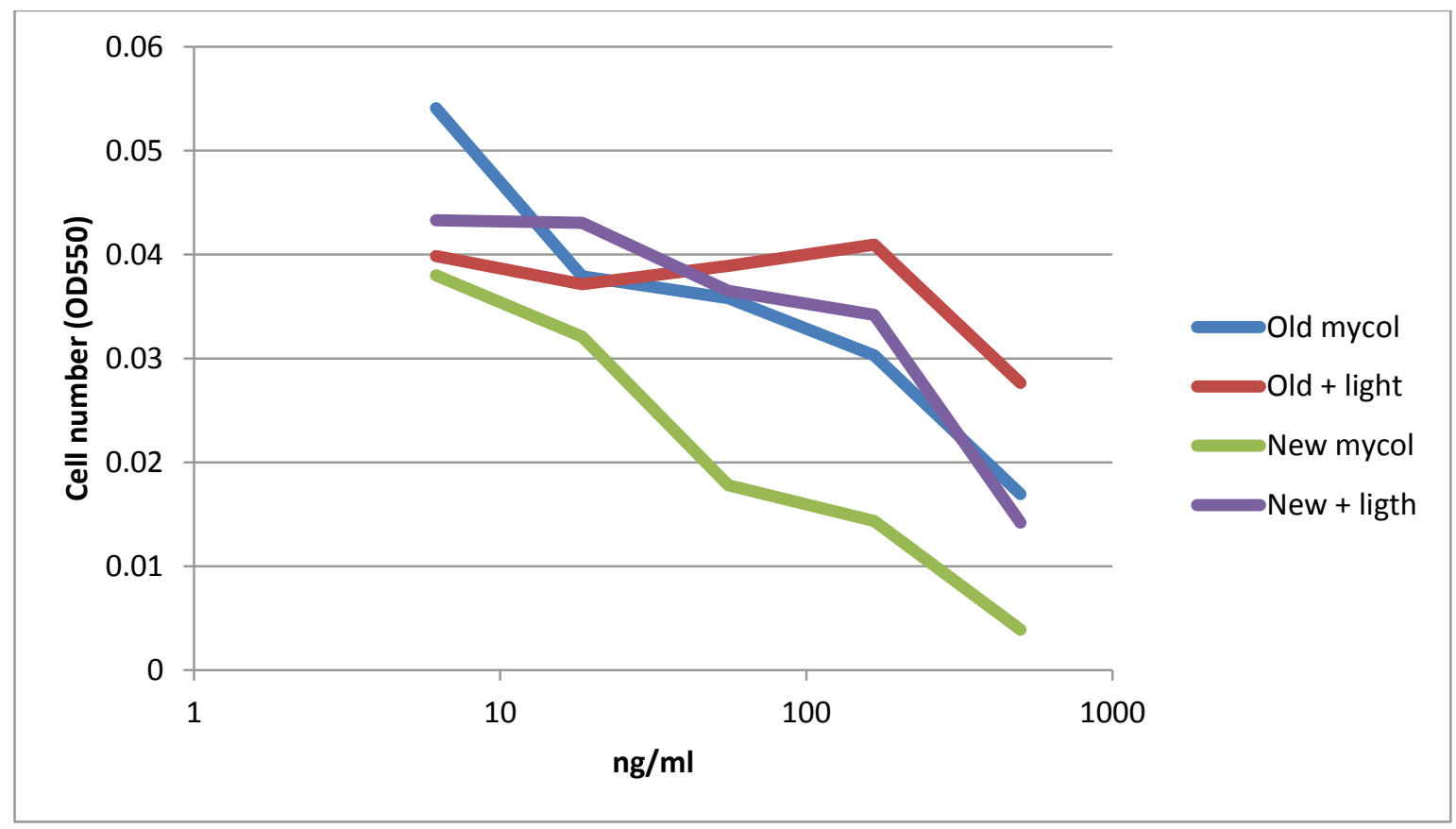

Figure 31. The Effect of Two Mycolactone Lots on Human Keratinocytes

NOTES: Both mycolactone lots were archived as thin films demonstrating mycolactone suffers significant detoxification while stored as a film. The time differential between batches was 131 days. 


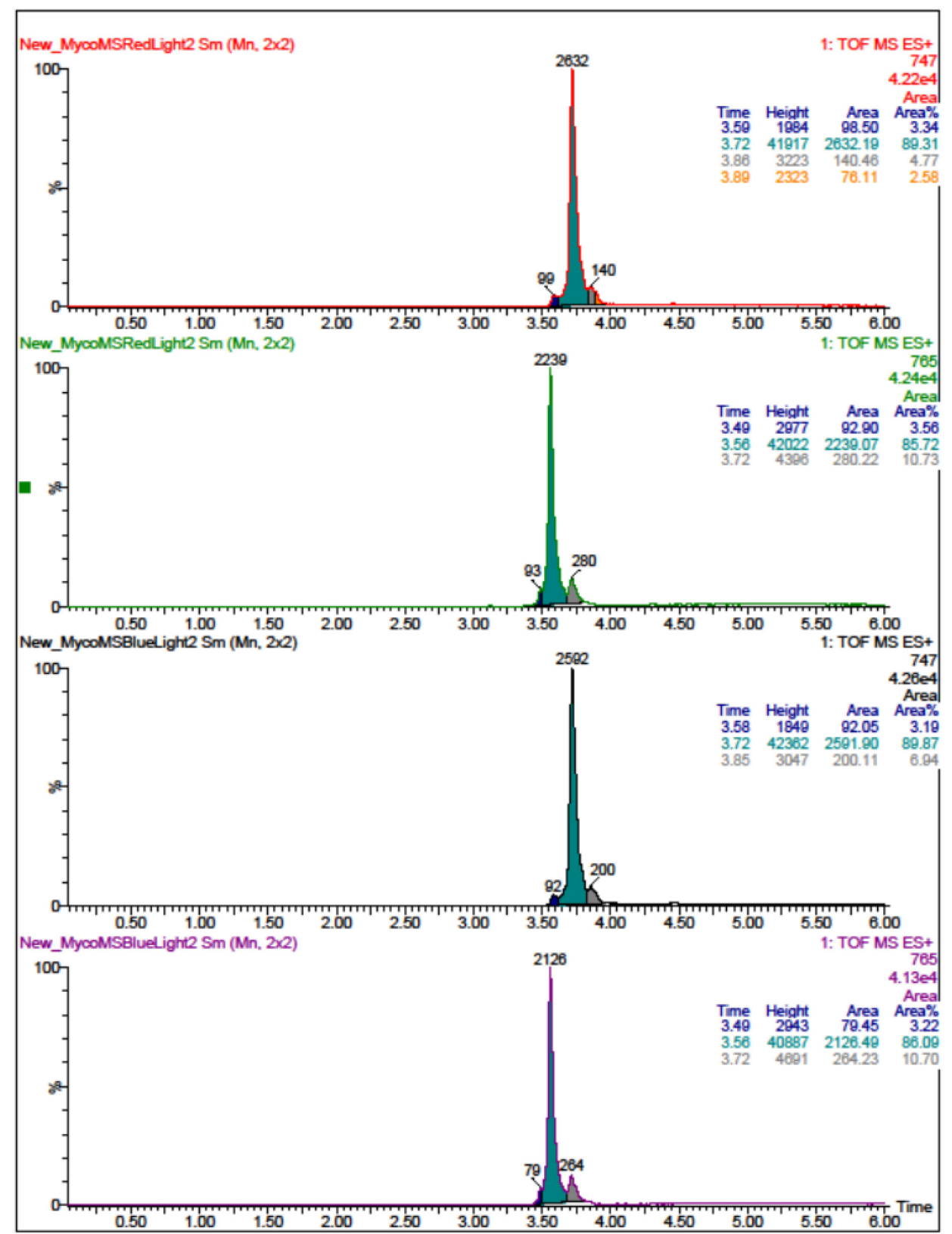

Figure 32. Analysis of Mycolactone Stability via Differential Red (Top Set) and UV (Bottom Set) Light Flow Spectroscopy of m/z 747 (Upper) and m/z 765 (Lower)

NOTE: No significant photoablation was detected in the UV/Vis flow cell exposed samples. 
classification of mycolactone subspecies, though we were unable to detect any significant mycolactone degradation products (Marion also failed to report any significant mycolactone photodegradation products and instead worked to assay primarily through spectroscopic means).

The Marion article also suggests a few improvements may be made to our framework. As our mycolactone efforts are shared by both national and international collaborators, the decision was made to store, assay and ship our mycolactone stocks as thin films. The reasoning was thin films would both reduce the accidental dissemination of highly toxic materials as well as greatly simplify the process of shipment. (The international shipment of highly toxic liquids is an extremely laborious and expensive process) Marion found storage as a thin film to be the least appropriate means of mycolactone archival due to proposed dehydration effects, mycolactone was found to be most amenable to archival in ACN at low concentrations.

While the article makes no effort to differentiate between the photoablative and chemical processes responsible for mycolactone's amazingly short half-life as a film, it will be interesting to determine if variations in our production and storage processes persists after transitioning to solution based archival.

Buruli ulcer, the human affliction associated with the mycolactone excreted by $M$ ulcerans as it attempts to evade the immune responses of its unwilling host, is an affliction largely borne by developing nations in tropical regions. While our initial laboratory efforts were simply concerned with culturing ulcerans for the production of mycolactone as a chemical probe, a number of our collaborators sought to leverage our knowledge in the effort to combat Buruli. The chemotherapeutic treatment for Buruli ulcer is the combinative administration of rifampicin and streptomycin, the intramuscular administration route and relatively short shelf-life of these drugs preclude their clinical application in many parts of the world. As a number of early efforts to characterize mycobacterial secretions (including our own), observed a sensitivity to light. An effort was undertaken to evaluate sunlight's ability to detoxify (and possible reverse) mycolactone associated necrosis. Clinical investigations are currently being conducted by the Britton group in Ghana and initial results appear promising.

As noted prior as, the optimal storage conditions for the archival of mycolactone was recently published. ${ }^{112}$ Based upon these findings, our decision to store our mycolactone stocks as thin films should be reviewed. In light of the thin film stability studies I believe many of the variations observed through our purification and analysis effort were likely a product of the thin film and not wholly associated with photoablative effects, especially in the laboratory. Our next lot of ulcerans should be ready for harvest soon and I have already revised the separation protocols to account for the findings of Marion.

To aid in the efforts I would like to continue the evaluation of the in-vitro photomitigation of mycolactone in an attempt to decisively answer lingering questions. While mycolactone's unique polyene system is responsible for the majority of the compound's 
UV absorbance (especially at longer wavelengths), no laboratory has currently been able to identify the remaining mycolactone fragments post photoablation. While our sunlight exposure experiments illustrated the ablative effects of sunlight on mycolactone, I was unable to conclusively identify any ablative fragments. I now believe this experimental shortcoming to be a function of the thin-film as opposed to the ablative or analytical processes themselves. As Marion conclusively illustrated, even very (60 min) periods of thin film storage resulted in a very significant reduction in the abundance of the mycolactone. In the next iteration of our ongoing mycolactone production and archival efforts I will implement the dilute ACN storage and processing techniques Marion found to be so successful. The extraordinarily low UV cutoff of high purity ACN will enable me to carry out my photoablations while preserving the resultant fragments for HRMS analysis and elucidation.

While the previous aim seeks to determine the most suitable form of UV irradiation for mycolactone exposure and its ablative products, I would also like to investigate the implications of exposing Buruli ulcer to UV and visible irradiation. Initial ulcerans infection presents as a small, seemingly insignificant, dermal papules and over an order of weeks to months slowly develops into a necrotic ulcer with germinal infiltration ${ }^{114}$. M ulcerans subcutaneous implications are largely based upon the observation that many of the most severe infections are often quite painless, presumably due to the toxin invading and destroying the deeply distributed pacinian corpuscles (nerve terminals housed deeply in the dermis) The question to me is, as the ulcerans is able to slowly encroach upon the human dermis, what form of irradiation would serve to deactivate deeply distributed mycolactone while preserving the patient's already compromised skin? As the susceptibility of human dermal tissues to various types of nonionizing irradiation is known, the question is how to evaluate the UV penetration of Buruli lesion. Collaborations are currently underway to tease out the response of dermal infection to irradiation in Ghana via surrogate in guinea pig. I believe these data in combination with my ablation findings will aid in the effective development of low cost phototherapies for ulcerans infection.

In this way I believe our laboratory can continue to provide high purity mycolactone stocks to our international collaborators and in our own little way help to curb the global affliction of Buruli ulcer. 


\section{Experimental Methods}

The experimental methods developed throughout this chapter are outlined below.

\section{SchuS4 Lipid Methods}

\section{Total Lipid Extraction}

ASL Lipid Extracts were generously provided by the Bosio laboratory.

\section{Fractionation of Cell Wall Associated Lipids (Modified Folsh)}

A solution of $\mathrm{MeOH} / 0.3 \% \mathrm{Aq} . \mathrm{NaCl}(100 / 10)(5 \mathrm{~mL})$ and Pet. Ether $(2.5 \mathrm{~mL})$ was added to lipid crude and stirred at $50^{\circ} \mathrm{C}$ for $30 \mathrm{~min}$ then centrifuged to form a partition. The organic layer was decanted and additional Pet. Ether $(2.5 \mathrm{~mL})$ was added to the remaining lipid for an additional extraction. The organic layers were combined and dried under a stream of nitrogen. A solution of $\left(\mathrm{CHCl}_{3} / \mathrm{CH}_{3} \mathrm{OH} / 0.3 \% \mathrm{NaCl}(90 / 100 / 30)(2.5\right.$ $\mathrm{mL}$ ) was added to the lipid containing vial and again stirred, extracted and partitioned. The organic layer was decanted and $1 \mathrm{~mL}$ of a solution of $\left(\mathrm{CHCl}_{3} / \mathrm{CH}_{3} \mathrm{OH} / 0.3 \% \mathrm{NaCl}\right.$ $(50 / 100 / 40)$ was added to the lipid stirred, extracted and partitioned. The organic layer was again removed and the extraction was repeated. The organic layers were pooled and dried. $2.5 \mathrm{~mL} \mathrm{CHCl}_{3}$ and $2.5 \mathrm{~mL} 0.3 \%$ Aq. $\mathrm{NaCl}$ were added to the remaining lipid mixture and briefly stirred then allowed to partition. Both layers were collected and brought to dryness under nitrogen stream.

\section{Fatty Acid Methyl Esterification}

Methanolic $\mathrm{HCl}$ was prepared by adding 7 drops $(250 \mu \mathrm{L})$ of acetyl chloride to 5 $\mathrm{mL}$ of very dry $\mathrm{CH}_{3} \mathrm{OH}$ in the fume hood. $\mathrm{HCl}-\mathrm{MeOH}(2 \mathrm{~mL})$ was added to $2 \mathrm{mg}$ of dry crude lipid and sample stirred to allow for dissolution. The sample was allowed to react overnight, and then dried under a stream of nitrogen. Hexane $(2.5 \mathrm{~mL})$ and $\mathrm{H}_{2} \mathrm{O}(2.5 \mathrm{~mL})$ were added to the vial to from a partition and the layers were stirred rapidly for 20 minutes. The hexane layer was removed and additional hexane $(2.5 \mathrm{~mL})$ was added and stirred. The organic extracts were dried in vacuo, resuspended in $\mathrm{CH}_{3} \mathrm{OH}$ and analyzed via $\mathrm{GC} / \mathrm{MS}$.

\section{GC/MS FAME Analysis}

GC/MS analyses were performed on an Agilent 5975c Series GC/MSD. All samples were diluted in $\mathrm{CH}_{3} \mathrm{OH}$ prior to injection. An Agilent DB-225 (30m x 250 $\mu \mathrm{m} x$ $0.25 \mu \mathrm{M}$ ) silica capillary column was utilized via thermal gradient of $90^{\circ} \mathrm{C}$ for $3.5 \mathrm{~min}$, 
then $4^{\circ} \mathrm{C} / \mathrm{min}$ to $220^{\circ} \mathrm{C}$ for $7 \mathrm{~min}$ for a total run time of 43 minutes. Detection was achieved via FID and MS. MS was configured and calibrated for "high gain" mode with a gain factor of 15 , a $5 \mathrm{~min}$ solvent delay and was scanned over $50-500 \mathrm{~m} / \mathrm{z}$. The EI source was maintained at $230^{\circ} \mathrm{C}$ while the quadrupole was heated to $150^{\circ} \mathrm{C}$.

\section{HPLC Fractionation and Analysis}

All samples were dissolved in GC/MS grade $\mathrm{CH}_{3} \mathrm{OH}$ and transferred to autosampler vials. Separations were achieved on a Phenomenex Luna $5 \mu$ C18(2) $(250 \mathrm{~mm}$ x $10 \mathrm{~mm} \times 5 \mu \mathrm{m}$ ) at room temperature, flowing $3.5 \mathrm{~mL} / \mathrm{min}$ of $\mathrm{H}_{2} \mathrm{O}+0.1 \%$ Formic acid (A) and $\mathrm{ACN}+0.1 \%$ Formic Acid (B). The first 12.5 minutes were maintained at $10 \%$ organic followed by a linear ramp to $95 \%$ organic over 45 minutes then held at high organic for an additional 12.5 minutes resulting in a 1 hour total chromatographic elution. All fractions were collected in glass tubes then turbovaped to dryness. The samples flagged through UV and ELSD were reanalyzed via UPLC/MS. Fractions found to contain lipid moieties were divided 1:10 for bioanalysis and archival respectively.

\section{UPLC/MS Analysis}

LC/MS Analyses were performed on both a Waters Acquity UPLC-TQD and a Waters Acquity UPLC-qTOF. All dry analytes were solvated in $\mathrm{CH}_{3} \mathrm{OH}$ and filtered prior to analysis; liquid analytes were diluted in the same and filtered. Separations were achieved at $0.5 \mathrm{~mL} / \mathrm{min}$ via a 5 minute gradient elution from $95 \% \mathrm{H}_{2} \mathrm{O}+0.1 \%$ Formic Acid to $95 \%$ ACN $+0.1 \%$ Formic Acid as follows. The first 30 seconds were held at the starting conditions then ramped to $40 \%$ organic over two minutes followed by a ramp to $95 \%$ organic an additional two minutes then followed by a 30 second stop at the $95 \%$ organic before returning to re-equilibrate. A Waters Acquity C18 BEH $1.7 \mu$ x $50 \mathrm{x}$ $3.0 \mathrm{~mm}$ column was maintained at $40^{\circ} \mathrm{C}$ through-out the elution series. Detection was achieved via PDA and MS.

\section{NMR Analysis}

All NMR samples were solvated in $600 \mathrm{uL}$ of $\mathrm{CDCl}_{3} .{ }^{1} \mathrm{H}$ spectra were acquired via a Spectrospin automated $400 \mathrm{MHz}$ Bruker spectrometer, while $2 \mathrm{D}$ data were acquired on a Varian Inova spectrometer operating at $600 \mathrm{MHz}$.

\section{Flash Chromatographic Separation of Crude Lipid}

Crude lipid extracts were solubilized in $1: 1 \mathrm{CHCl}_{3}: \mathrm{CH}_{3} \mathrm{OH}$ through the aid of sonication. $1 \mathrm{~g}$ of dry chromatographic silica was added to the lipid solution and brought to dryness via rotovap. The lipid loaded silica was transferred to a previously equilibrated $12 \mathrm{~g}$ Biotage HP-Sil SNAP flash chromatographic cartridge. Elution was achieved via a gradient separation from $\mathrm{CHCl}_{3}$ to $3: 1 \mathrm{CHCl}_{3}: \mathrm{CH}_{3} \mathrm{OH}$ over 15 column volumes at a flow 
rate of $12 \mathrm{~mL} / \mathrm{min}$. Detection was achieved via UV absorbance at 220 and $254 \mathrm{~nm}$. All fractions were collected in glass tubes and were brought to dryness via turbovap.

\section{Mycolactone Methods}

\section{Isolation of Mycolactone from Ulcerans}

M. ulcerans was grown in solid 7H11 plates for 14-16 weeks. Colonies were manually selected and transferred into a PBS solution where they were harvested by centrifugation and again washed with PBS. All PBS was then removed and 5x weight/volume of 5:1 EtOH: $\mathrm{H}_{2} \mathrm{O}$ was added and extracted for one hour at $37^{\circ} \mathrm{C}$. The solution was then clarified via centrifugation and the supernatant decanted. The extraction process was repeated until the EtOH solution no longer retained a yellow coloration $(2-3 x)$. The extracts were brought to dryness via rotovap. The dry materials were resolvated in acetone with the aid of sonication and filtered to remove remaining cellular debris then again brought to dryness via rotovap

\section{Sunlight Induced Photodetoxification of Mycolactone}

$1 \mathrm{~mL}$ of mycolactone solution $(500 \mu \mathrm{L} / \mathrm{mL})$ was applied to transparent plastic plates. The plates were slowly rotated to allow the formation of a thin film and randomly assigned to two groups. The control group was carefully wrapped in parafilm then aluminum foil. The exposure group was covered with a transparent quartz slides, both sets of samples were carefully transported outdoors on a sunny day near 1 PM and exposed to sunlight for periods of 30 minutes to 2 hours. The films were then removed via $\mathrm{CH}_{3} \mathrm{OH}$ and analyzed via $\mathrm{GC} / \mathrm{MS}$.

\section{HPLC Fractionation and Analysis}

All samples were dissolved $\mathrm{CH}_{3} \mathrm{OH}$. Separations were achieved on a Phenomenex Luna $5 \mu \mathrm{C} 18(2)(250 \mathrm{~mm} \times 10 \mathrm{~mm} \times 5 \mu \mathrm{m})$ at room temperature, flowing $3.5 \mathrm{~mL} / \mathrm{min}$ of $\mathrm{H}_{2} \mathrm{O}+0.1 \%$ Formic acid (A) and ACN+ 0.1\% Formic Acid (B). The first 12.5 minutes were maintained at $10 \%$ organic followed by a linear ramp to $95 \%$ organic over 45 minutes then held at high organic for an additional 12.5 minutes resulting in a 1 hour total chromatographic elution. All fractions were collected in glass tubes then were turbovaped to dryness. The samples flagged through UV and ELSD were reanalyzed via UPLC/MS. Fractions found to contain mycolactone were selected for archival.

\section{Flash Chromatographic Separation of Crude Lipid}

Crude lipid extracts were solubilized in 1:1 $\mathrm{CHCl}_{3}: \mathrm{CH}_{3} \mathrm{OH}$ through the aid of sonication. $1 \mathrm{~g}$ of dry chromatographic silica was added to the lipid solution and brought 
to dryness via rotovap. The lipid loaded silica was transferred to a previously equilibrated $12 \mathrm{~g}$ Biotage HP-Sil SNAP flash chromatographic cartridge. Elution was achieved via a gradient separation from $\mathrm{CHCl}_{3}$ to $3: 1 \mathrm{CHCl}_{3}$ : $\mathrm{CH}_{3} \mathrm{OH}$ over 15 column volumes at a flow rate of $12 \mathrm{~mL} / \mathrm{min}$. Detection was achieved via UV absorbance at 320 and $360 \mathrm{~nm}$. All fractions were collected in glass tubes and were brought to dryness via turbovap.

\section{LC/MS Analysis}

LC/MS Analyses were performed on both a Waters Acquity UPLC-TQD and a Waters Acquity UPLC-qTOF. All dry analytes were solvated in $\mathrm{CH}_{3} \mathrm{OH}$ and filtered prior to analysis; liquid analytes were diluted in the same and filtered. Separations were achieved at $0.5 \mathrm{~mL} / \mathrm{min}$ via a 5 minute gradient elution from $95 \% \mathrm{H}_{2} \mathrm{O}+0.1 \%$ Formic Acid to $95 \%$ ACN $+0.1 \%$ Formic Acid as follows. The first 30 seconds were held at the starting conditions then ramped to $40 \%$ organic over two minutes followed by a ramp to $95 \%$ organic an additional two minutes then followed by a 30 second stop at the $95 \%$ organic before returning to re-equilibrate. A Waters Acquity C18 BEH $1.7 \mu$ x 50 x $3.0 \mathrm{~mm}$ column was maintained at $40^{\circ} \mathrm{C}$ through-out the elution series. Detection was achieved via PDA and MS. 


\section{CHAPTER 3. HRMS BASED METABOLIC PROFILING OF COMPOUNDS FOR THE TREATMENT OF TUBERCULOSIS}

\section{Introduction to Drug Resistance}

Drug resistance is a general term used to indicate a chemotherapeutic agents loss of efficacy for a particular indication. Bacterial resistance occurs when a bacterial pathogen is able to survive exposure to a chemotherapeutic agent to which it previously was susceptible. Numerous bacterial resistance mechanisms are known to exist arising from mutation, natural selection and external evolutionary stresses ${ }^{115}$ (i.e., exposure to antimicrobial agents). It is well documented that natural product based antimicrobial agents as well as the bacterial genes now known to confer resistance have existed since antiquity. ${ }^{116}$ Unfortunately, the rampant overuse of antibiotics in both human and domesticated livestock populations as seen over the last half century has led to a new and disturbing phenomenon of greatly increased incidences of drug resistant pathogens in the clinic. ${ }^{117}$

This overwhelming increase in the drug related selective pressures placed upon pathogenic bacteria has led to the development of highly virulent bacterium, resistant to many of the most common anti-infective compounds on which modern human society has come to depend. ${ }^{118}$ Numerous efforts to decrease the antimicrobial burden on animal food-stocks are currently underway, while most prescribers have voluntarily enlisted in antibiotic stewardship programs. ${ }^{119}$ While there is a near universal consensus the efforts to limit the selective pressures placed upon bacteria are both timely and necessary. The majority of the front-line antibiotics currently in clinical use have continued to see dramatic reductions in efficacy ${ }^{120}$ and there is a major concern that antibiotic stewardship efforts are being undermined by poor drug administration practices in developing nations. ${ }^{121}$

It is becoming increasingly clear that antibiotic stewardship alone is not sufficient to curb the ever increasing rate of bacterial resistance. ${ }^{122}$ New antimicrobial chemotherapeutic agents are desperately needed to replace our aging antibacterial arsenal. Unfortunately, the 1980-1990's witnessed a steep decline in both the monetary investment and productivity of many large-scale pharmaceutical companies as numerous anti-infective programs were shuttered in pursuit of therapies offering higher returns. ${ }^{123}$ While this decade has witnessed something of a renewed interest in the commercial investigation of antibiotic small molecules, the battle currently appears to be favoring the pathogenic organisms over their human hosts.

\section{New Compounds Needed to Combat Antimicrobial Resistance}

As ever increasing rates of resistance continue to limit the clinical efficacy of existing antimicrobial compounds, the need for new drugs becomes all the more urgent. As viewed through the lens of bacterial resistance another aspect of classical antibiotic 
development becomes troubling. While many antibiotic agents exist on the market today, the vast majority of them exist as only minor modifications to previously approved drugs. This limited diversity in the anti-infective drug space makes not only resistance but crossresistance a persistent threat. ${ }^{124}$ Over $70 \%$ of the antibiotic agents approved by the FDA since 1982 have been modifications of only two chemotherapeutic classes, quinolones and beta-lactams. ${ }^{125}$ Furthermore, the golden age of antibiotic research (1940-1970) witnessed only 14 classes of novel antimicrobial scaffolds approved for use in humans. ${ }^{126}$ Only five truly novel scaffolds have been approved since; mupirocin, pleuromutilins, lipopeptides, oxazolidinones and glycylcyclins. The question now is how does a scientist go about discovering a previously unknown chemical scaffold which may be synthetically manipulated into a chemotherapeutic agent?

The answer is both complex and multifaceted; some have chosen to screen large bio-diverse libraries, others have relied on structure-based design while still others have chosen to utilize combinatorial approaches. ${ }^{127}$ At this time there appears to be no truly right or wrong answer. One critical deficiency in many of the current antimicrobial drug development pipelines is the early preclinical investigation of an agent's bacterial metabolic stability. Preclinical MS/MS screening provides scientists the ability to preselect drug candidates exhibiting excellent metabolic stability in the host and with respect to degrading enzymes found in some bacteria. Work in this chapter seeks to utilize high resolution mass spectrometry as a tool to investigate how both bacteria and the human body modify and eventually eliminate therapeutic agents modeled upon both spectinomycin and nitrofurantoin, and seeks to apply this knowledge towards the eventual synthesis of novel synthetic scaffolds.

\section{Introduction to Antitubercular Chemotherapeutic Agents}

A number of commercially available antibiotics are currently utilized in the treatment of tuberculosis worldwide. Antitubercular drugs are commonly subdivided into first-line and second-line agents, with the first-line agents generally conferring fewer offtarget (side) effects. ${ }^{128}$ The first-line class is composed of rifampin, isoniazid, pyrazinamide, ethambutol and streptomycin. ${ }^{129}$ The second-line agents generally include kanamycin, cycloserine, ethionamide, capreomycin and para-aminosalicyclic acid. ${ }^{130}$ While individual therapies of first and second-line agents may be capable of inhibiting the proliferation of tuberculosis, TB mono-therapies have been largely discontinued due to the rapid conference of drug resistance. ${ }^{131}$ The current $\mathrm{CDC}$ recommendation for $\mathrm{TB}$ infection is RIPE ${ }^{132}$ (rifampin, isoniazid, pyrazinamide, ethambutol) coadministration for two months followed by an additional 4-6 months of rifampicin and isoniazid. In cases of suspected multidrug resistant tuberculosis (MDR-Tb) RIPE therapy is recommended for 18-24 months. ${ }^{133}$ While RIPE therapy confers a near $95 \%$ cure rate when administered as directed, the complicated dosage form (up to 20 pills daily) often results in poor patient compliance and subsequent manifestation of MDR-TB. ${ }^{134}$ New TB drugs are desperately needed both to replace chemotherapies relegated through resistance as well as to offer increased patient compliance through reduced dosage frequency. Encouragingly there are a number of new TB chemotherapies currently in clinical trials, including; LL-3858, PA- 
824, OPC-67683 and KRM-1648(Rifalzil), which soon may afford adjunctive administration to RIPE, potentially reducing the duration of RIPE therapy and associated patient non-compliance.

\section{Introduction to M. tuberculosis Pathogenicity}

The wide spread use of antitubercular agents coupled with poor patient compliance and HIV co-infection has led to a drastic increase in the instance of drug resistant infections seen in the clinic over the past twenty years. ${ }^{135}$ While numerous "best practice" strategies seek to limit the evolutionary pressure collectively placed upon pathogenic bacteria such as tuberculosis, new antitubercular compounds will be required to effectively combat infections in the near future. ${ }^{136}$ The key to the development of any new antibacterial agent is an advanced understanding of the mammalian immune system and of the strategies tuberculosis employs to evade it. The human immune system and infectious tuberculosis bacteria have co-evolved together, with each organism seeking to develop more effective countermeasures against the other. On the front line of this everpresent conflict are the phagocytes seeking to envelop and destroy evading microorganisms and obligate human pathogens such as M. tuberculosis which seek to corrupt these biochemical machines for their own gain. ${ }^{137}$

Phagocytes are white blood cells tasked with internalizing and destroying invading organisms and are often loosely grouped based upon their affinity for phagocytosis. The most aggressive or professional phagocytes (macrophages, monocytes, neutrophils dendritic and mast cells) are specially equipped to ingest and destroy large cells including bacteria. ${ }^{138}$ Phagocytes are capable of detecting pathogens both indirectly through opsonins (commonly $\operatorname{IgG}$ ) and directly through the use specific receptors presensitized to antigens expressed upon bacterial cell walls. Once an invading bacterium has trigger the phagocyte, a phagosome is formed through the remodeling of actin aided by phosphoinositides and myosin $\mathrm{X} .{ }^{139}$

The ultimate goal of this remodeling is to move the bacterium from a free environment to a completely encapsulated compartment of the phagocyte in a process known as phagosome sealing. Once the phagosome is complete the process of maturation may begin, in the maturation stage a number of lysosomes are fused with the newly formed phagosome and the lumen is slowly acidified. ${ }^{140}$

As the early phagosomal lumen continues to acidify, additional biochemical processes are responsible for beginning to confer antibacterial properties to the phagocyte through the activation of numerous GTPases and SNARE proteins. Once the $\mathrm{pH}$ falls below about 6.0, proton-pumping V-ATPases are recruited to further drive the luminal acidification in this so called late phagosome. ${ }^{141}$ The late phagosome continues to be enriched through the incorporation of proteases and liposomal proteins, ultimately resulting in the creation of phagolysosome. An organelle specifically created for its devastating antimicrobial properties. Phagolysosomes, rely on a number of powerful weapons to completely destroy the encapsulated microorganism; the foremost being a 
highly acidic phagosome ( $\mathrm{pH} 4.0$ - 5.0) as well as antimicrobial peptides, proteases, iron scavengers (such as lactoferrin) and NADPH family oxidases. ${ }^{142}$

While the immune system has evolved powerful defenses against most bacteria, some bacterial pathogens, including $M$. tuberculosis, have developed advanced strategies seeking to subvert or attenuate a normal immune response. Though numerous microbial organisms have devised systems to elude the immune system, there exist a small number of "professional" pathogens capable of not only surviving inside of the phagosome but thriving there.

Mtb is one of the few successful intracellular bacterial pathogens with the ability to avoid many of the destructive mechanisms of the phagocytic compartment, primarily by preventing the phagosome from fully maturing. While the $\mathrm{pH}$ of the native phagolysosome often reaches 4.5, the phagosomal acidification of Mtb infected cells stalls well before they reach toxic levels ${ }^{143}(\mathrm{pH} 6.4)$. While there exists some debate as to how the bacterium is able to effect maturation, a number of cell wall glycolipids seem to have been decisively implicated in the process. ${ }^{144}$ In concert with Mtb's ability deceive the phagosome, the bacterium is also able to enter a number of replicative states to further its ability to survive in a potentially hostile environment. Once the bacterium has successfully evaded the body's initial defensive efforts it begins to proliferate rapidly. This period of rapid proliferation, primarily concentrated to the alveolar macrophages, continues until the host is able to mount an acquired immune response. ${ }^{145}$

At this point in the infection the bacterial count begins to plateau, many of the initial overt signs of the disease fade and the pathogen enters a stage of immune mediated control. ${ }^{146}$ While the exact cause of Mtb's perceived stasis remains unknown, many believe hypoxia remains primarily responsible for control of bacterial growth in the chronic stages of persistent infection. ${ }^{147}$

The immunological responses mounted against the invasion of $\mathrm{M}$. tuberculosis and the pathways the pathogen employs to subvert them are complex. Mtb has evolved the ability to evade the early immune defenses targeted against it, as well as to package itself into a defensive granuloma once the body is capable of mounting a significant response. ${ }^{148}$ These traits have enabled Mtb to become a persistent pathogen without the reliance upon an animal reservoir, and have greatly complicated the treatment of persistent infection. However, it is hoped that a greater understanding of the human immune system and the mechanisms Mtb uses to usurp it will prove instrumental in the development of new chemotherapeutic agents against this enduring threat. To this end, this work seeks to provide a greater understanding of the host/pathogen symbiosis in two important ways. First, through the application of HRMS tools to investigate the small molecule bacterial metabolites (lipids and mycolactone) responsible for perturbing the innate immune response and secondly, to probe the chemical processes that serve to influence the metabolism of anti-infective chemotherapies. 


\section{An Overview of MS Metabolic Profiling}

The initial discovery and subsequent testing of any new therapeutic agent is an extremely expensive and time-consuming venture. Current estimates place the cost of deploying a novel therapeutic compound at around one billion dollars with an average time to market of just over twelve years. ${ }^{149}$ To remain competitive, any serious venture must have some framework on which to evaluate the many hundreds if not thousands of hits often generated by modern cell based screening of large chemical libraries. Add to this cost the troubling high rate of attrition, over $70 \%$ off all new chemical entities (NCEs) ultimately fail and the potential impact to both the pharmaceutical industry and academic researcher is severe. ${ }^{150}$ Beyond these initial expenses, one must also consider the potential implications to the patient, as adverse drug reactions (ADR) due to unfavorable drug metabolism and pharmacokinetics (DMPK) is a leading cause of NCE withdrawal.

From this flood of data researchers must ultimately select a small number of lead compounds to undergo a battery of costly test aimed at evaluating a potential NCE's efficacy as well as its' toxicological profile. A number of relatively new analytical techniques have proven themselves indispensable in their ability to rapidly evaluate NCEs throughout the development and testing process. Nobilis states, ${ }^{151}$ high throughput liquid chromatography tandem mass spectrometric (LC-MS/MS) techniques coupled with medium throughput, high information content, liquid chromatography nuclear magnetic resonance spectroscopy (LC-NMR) have proven invaluable in their ability to quickly evaluate NCEs. LC-MS ${ }^{\mathrm{n}}$ techniques have proven applications throughout the evaluation of chemical leads. From early hit identification and sample quantification, through traditional adsorption-distribution-metabolism-excretion (ADME) workflows and finally onto the prediction of ADRs through the investigation of potentially reactive chemical species $^{152}$ (RCSs).

This work will primarily focus on the application of $\mathrm{LC}-\mathrm{MS}^{\mathrm{n}}$ techniques to the identification of metabolites as RCSs and the pharmacokinetic (PK) profiling of drug metabolism. Over countless years the human body has evolved defenses against the incursion of foreign or exogenous compounds, these xenobiotic agents are systematically functionalized through a series of chemical reactions leading to the increase of a toxins hydrophilicity and ultimately to its excretion. ${ }^{153}$ These biochemical reactions may be further subdivide into two metabolic phases; phase I, cytochrome P450 mediated biotransformations often seeking to insert a nucleophile (commonly hydroxyl) decreasing a compound's lipophobicity and inserting a site for the ultimate phase II conjugation and excretion of a drug through the urine. ${ }^{154}$ Given these transformations usually occur in a predictable manner and aided by advances in synthetic physical property prediction algorithms, scientists now have the ability to generate metabolic profiles of a lead therapeutic compound in advance of costly clinical trials. These projected PK profiles coupled with synthetic metrics of solubility, distribution, polar-surface-area (PSA) etc., may then be used in selecting chemical leads to continue through the development pipeline. $^{155}$ 
As noted earlier, the modern PK framework must be capable of handling the heavy workflows endemic to drug discovery laboratories, as such, tasks must be logically ordered so that rapid, automated (information poor) analysis may be used to guide more time consuming, information rich, steps. The initial classification of most NCE metabolites will often be conducted with the aid of the TQD, as a number of different experiments may be conducted in a highly efficient fashion. One of the most common experiments ${ }^{156}$ involves the operation of a single quadrupole (Q1) in the TQD. This single scan acquisition is used to rapidly provide data such as; ionization efficiency (the ability to detect positively or negatively charged molecular ions), nominal mass difference (mass of parent $+/$ - mass of analyte) and qualitative nitrogen content according to the "nitrogen rule". The so called nitrogen rule states that organic compounds containing exclusively hydrogen, carbon, nitrogen, oxygen, silicon, phosphorus, sulfur, and the halogens have either 1) an odd nominal mass that indicates an odd number of nitrogen atoms are present or 2) an even nominal mass that indicates an even number of nitrogen atoms are present in the molecular ion. ${ }^{157}$

This easily obtainable information is then used to target more advanced TQD experiments, these so called precursor-ion and product-ion scans rely on the systematic destruction of incoming molecular ions in the TDQ's collisional cell to create characteristic fragmentation patterns. In this way, these distinct molecular fingerprints may be used to identify various metabolic pathways; as in the neutral loss of $176 \mathrm{~m} / \mathrm{z}$ (the fingerprint of glucuronidation) or the precursor loss of $80 \mathrm{~m} / \mathrm{z}$ (indicative of sulfation). The data generated from scanning and collision induced analysis may then be used in the design of multiple reaction monitoring (MRM) assays, in which the fragmentation patterns of known metabolites are preselected, effectively eliminating background noise ${ }^{158}$. In this way MRM experiments can prove to be extremely valuable in the quantification of metabolic products, while a truly powerful technique, MRM experiments usually reside at the end of the TQD based workflow as the design of a meaningful MRM experiment can prove very time consuming. See Table of Characteristic Metabolic Transformations (Appendix A and Tables 3 and 4).

While the TQD remains the workhorse of most any metabolomics laboratory, recent advances in bench-top high resolution mass spectrometers (HRMS) have allowed many institutions access to the world of accurate mass spectrometry. ${ }^{159}$ HRMS data has proven extremely valuable for the predication of proposed chemical formula based upon a compounds isotopic elemental abundance or mass defect. Trace level metabolic pathways can now be rapidly elucidated based upon the mass defect observed between parent and metabolized products aided by the complimentary fragment data provided by TQD analysis.

\section{HRMS Stability Analysis of Spectinomycin Derivatives}

Our laboratory has an ongoing effort to synthesis new spectinomycin analogs in an effort to overcome the primarily, efflux pump mediated, resistance that precludes the parent compound from widespread clinical compatibility. Three of the lead compounds in our spectinomycin derivative series are Lee-1329 (Figure 33), Lee-1544 (Figure 34) and 


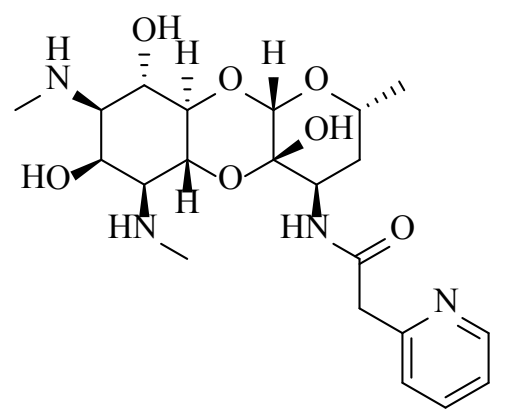

Figure 33. Structure of Lee-1329

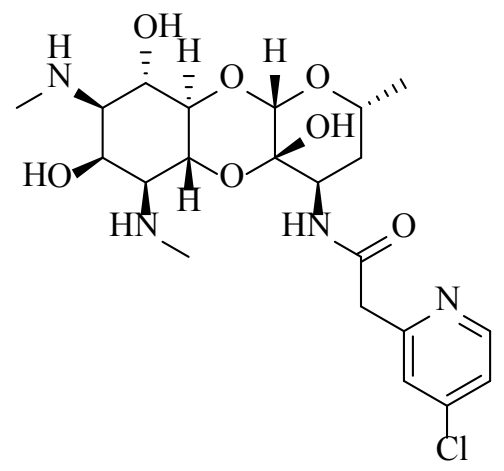

Figure 34. Structure of Lee-1544 
Lee-1599 (Figure 35). The identification of these metabolites was sought to guide further medicinal chemistry efforts and evaluate potential metabolite related toxicities and to study the potential for drug interactions. Using a careful structural analysis of these drugs a number of theoretical metabolic sites and products were proposed as illustrated for Lee1329 (Figure 36) and Lee-1544 (Figure 37). Based upon the initial findings HRMS experiments were designed to determine the exact nature of metabolic products both invivo and in-vitro. Initial microsomal stability studies indicated Lee-1329 and Lee-1544 were susceptible limited metabolic degradation. Preliminary rat liver microsomal phase-II metabolism stability data indicated Lee 1329, 1544 and 1599 did not serve as substrates for glucuronide conjugation nor were they significantly degraded through sulfoconjugation. However, primary screens indicated Lee 1544 was significantly metabolized phase-II glutathione conjugation.160,161

\section{MassLynx and HRMS Analysis of Microsomal Stability}

Preliminary data indicated both 1329 and 1544 were poor substrates for elimination via glucuronidation (Figure 38) or sulfo-conjugation (Figure 39) and these results were confirmed upon HRMS Analysis. The further investigation of GSH conjugation products was, however, more involved (Figure 40). The more nonpolar 1544 will be utilized to illustrate the applications of HRMS metabolic profiling. The first minor departure from the initial finding is that a global comparison of the parent drug across all microsomal samples indicated that while the concentration of parent drug was reduced in the GSH plates, 1544 was more stable than initially expected. Upon data analysis an extracted ion integration of the parent $1544(487.2 \mathrm{~m} / \mathrm{z})$ revealed that $91-93 \%$ of the parent 1544 remained unchanged depending on the particular extract analyzed.

Upon background subtraction and analysis the GSH analytes were found to contain two interesting highly polar compounds apparently related to the parent 1544 (487.2165 m/z, $1.43 \mathrm{~min})$. The GSH compounds contained two interesting co-eluting peaks at $0.51(308.1049 \mathrm{~m} / \mathrm{z})$ and $0.63(613.2486 \mathrm{~m} / \mathrm{z})$ minutes, respectively (Figures 41 and 42).

As numerous pathways exist to further detoxify GSH conjugation products the lack of the prototypical $\Delta 306 \mathrm{~m} / \mathrm{z}$ has been previously reported, especially in hydrophilic halogenated substrates. 162 Figure 43 illustrates a free GSH moiety while Figure 44 is the observed GSH-1544 Conjugation Product.

\section{MassLynx and HRMS Urinalysis of Lee Compounds}

Upon completion of the in-vivo studies, the program was advanced in an effort to elucidate the metabolic fate of 1544 in rats. Sprague Dawley rats were catheterized and dosed the investigational spectinomides intravenously at $10 \mathrm{mg} / \mathrm{kg}$ body weight. The urine was collected on six hour intervals for the first 12 hours, then on 12 hour intervals for an additional 60 hours. The crude urine was processed via centrifugation and $\mathrm{CH} 3 \mathrm{OH}$ 


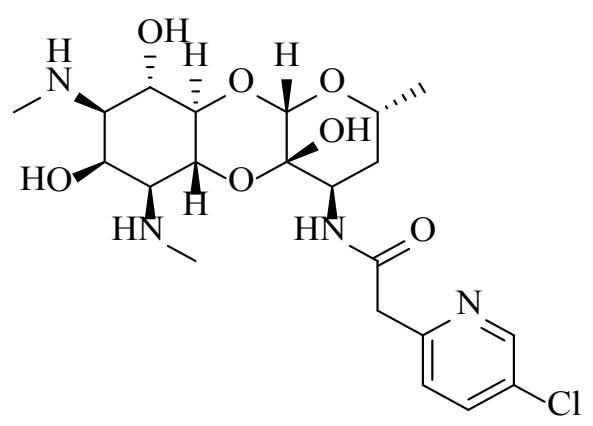

Figure 35. Structure of Lee-1599

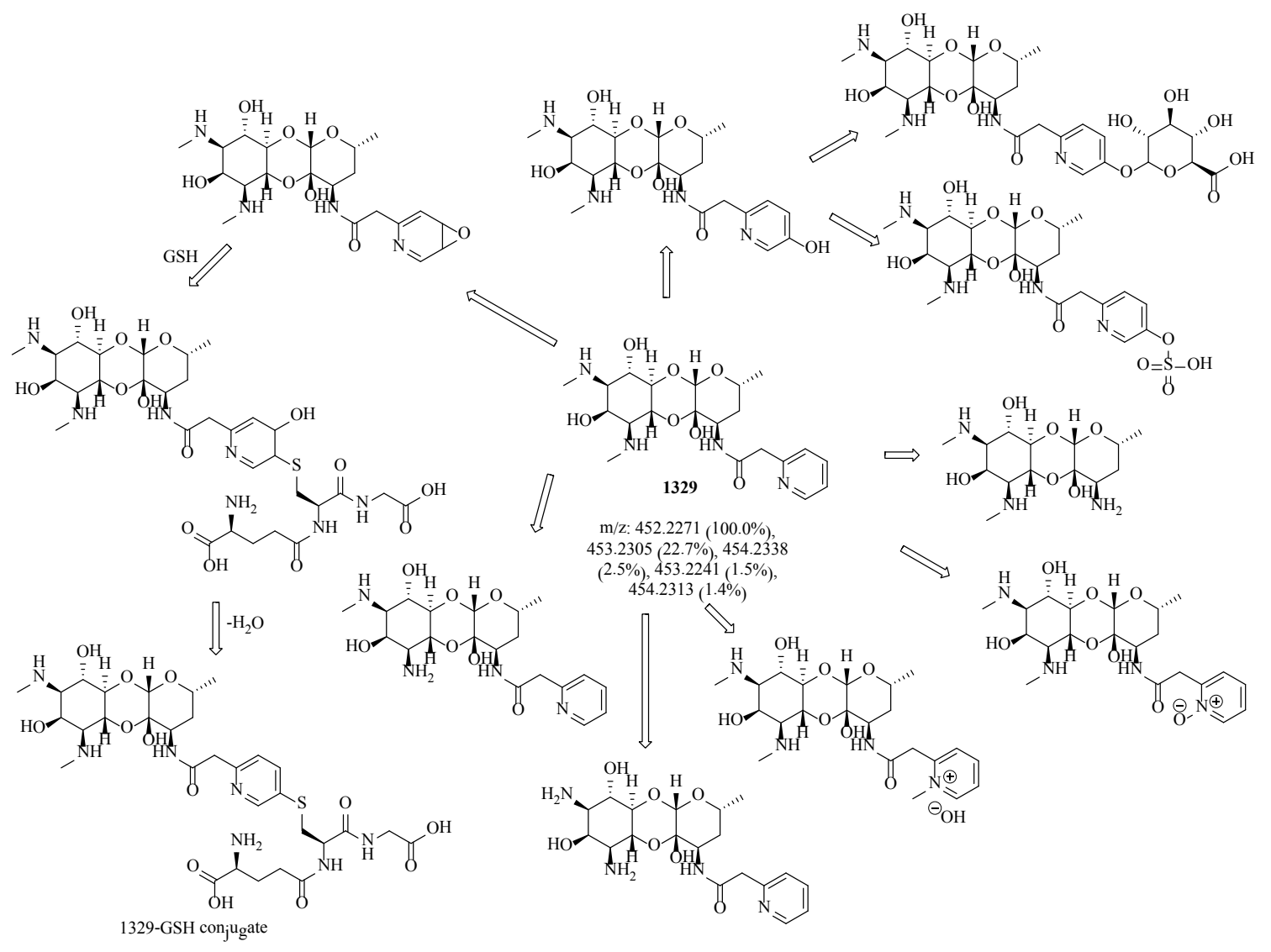

Figure 36. Possible Metabolites of Lee-1329 


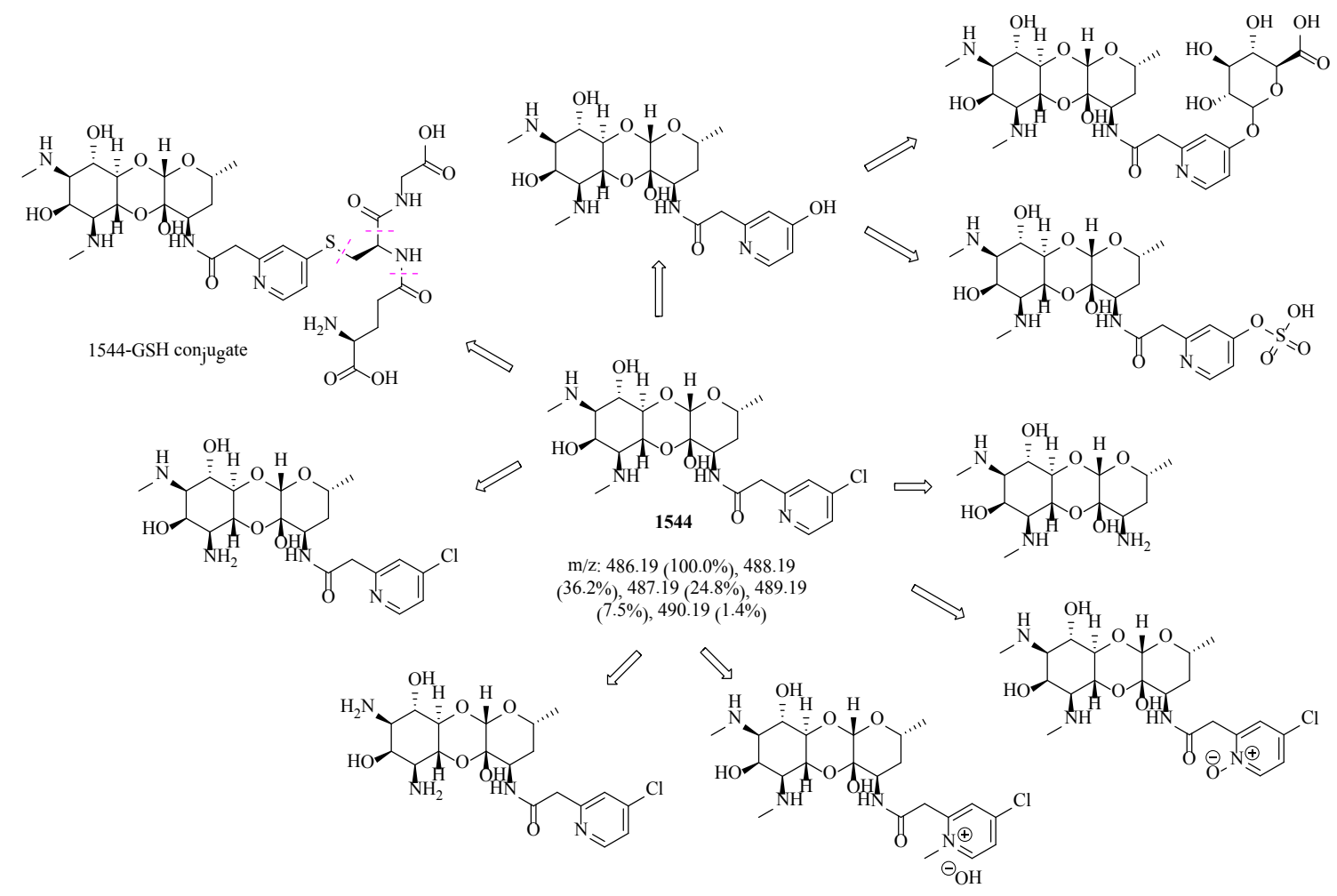

Figure 37. Possible Metabolites of Lee-1544 
$\%$ remaining of parent compound after Glucuronide conugation in Rat Liver $\mathbf{S 9}$

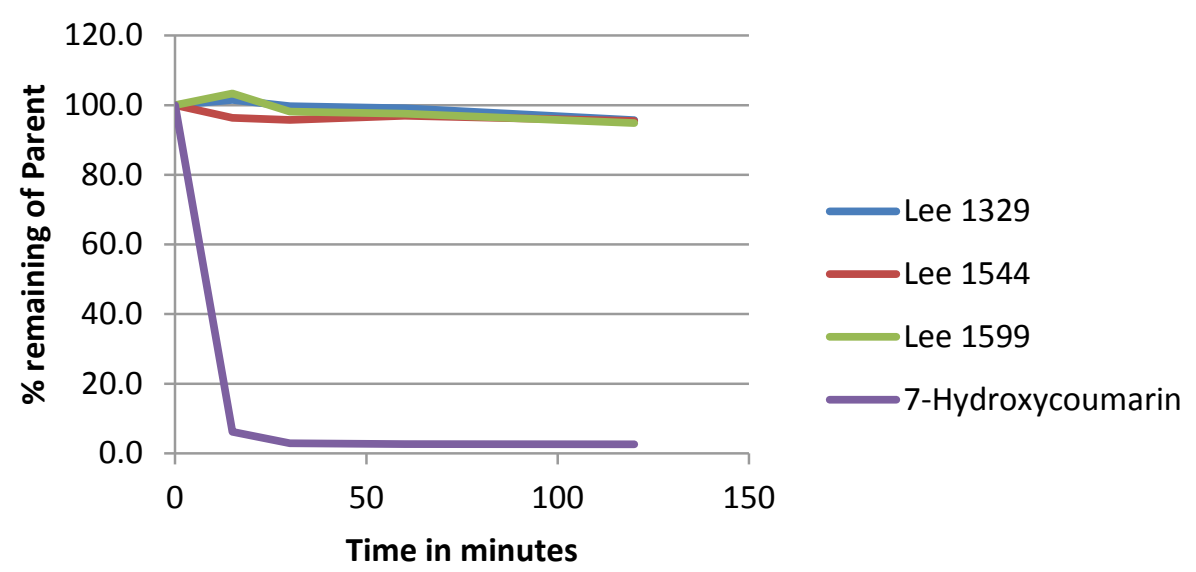

Figure 38. Preliminary Phase 2 Glucuronide Conjugation of Lee Compounds and Control in Rat Liver S9 Microsomes

NOTE: All Lee compounds appear highly stable. 
$\%$ remaining of parent compound after Sulfoconjugation in Rat Liver $\mathbf{S 9}$

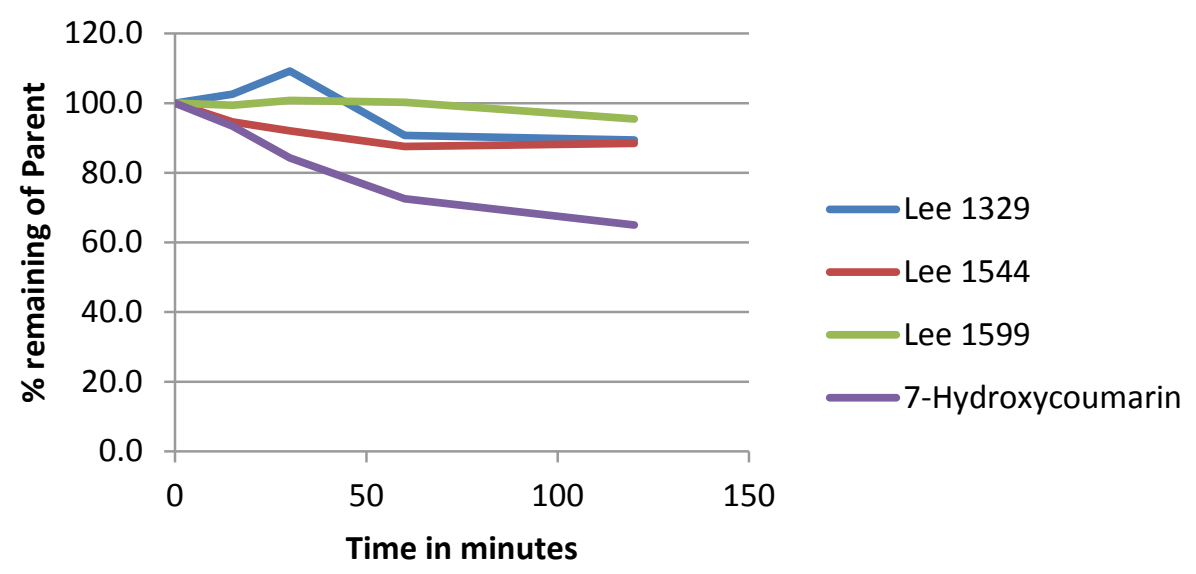

Figure 39. Preliminary Phase 2 Sulfo-Conjugation of Lee Compounds and Control in Rat Liver S9 Microsomes

NOTE: All Lee compounds appear highly stable. 
$\%$ remaining of parent compound after Glutathione conugation in Rat Liver S9

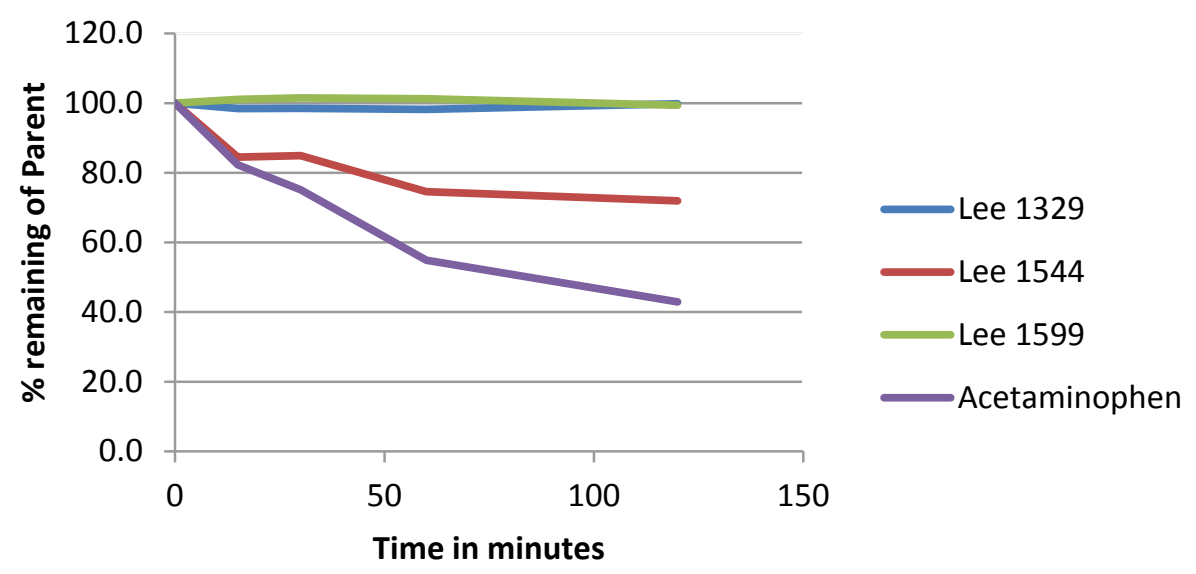

Figure 40. Preliminary Phase 2 Glutathione Conjugation of Lee Compounds and Control in Rat Liver S9 Microsomes

NOTE: Lee compounds 1329 and 1599 appear highly stable, while Lee 1544 suffered some metabolism. 


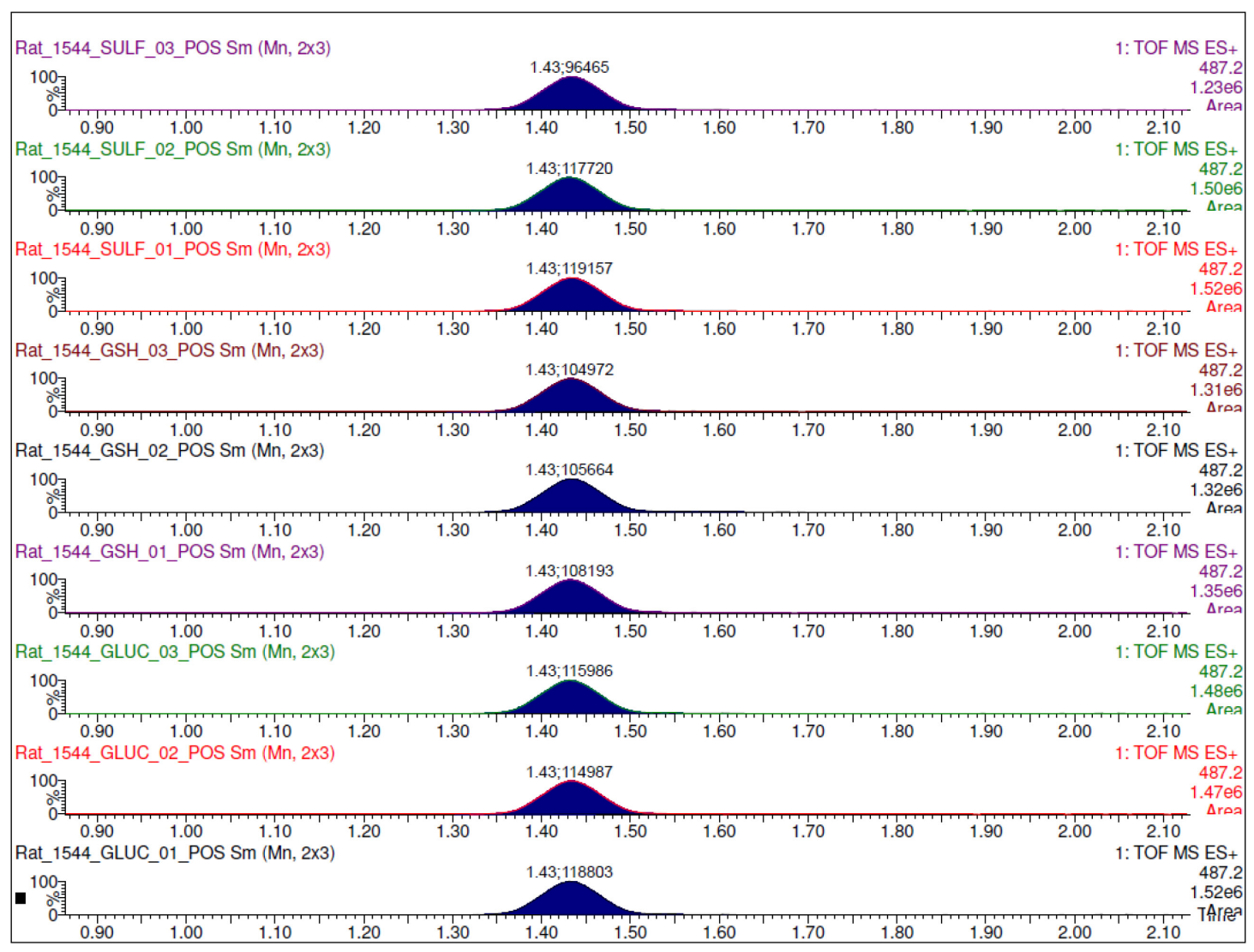

Figure 41. Extracted Ion Spectra of Lee 1544 Remains Unchanged at the Conclusion of Microsomal Incubation, Indicating 1544 Is Significantly More Stable than Initially Predicted 


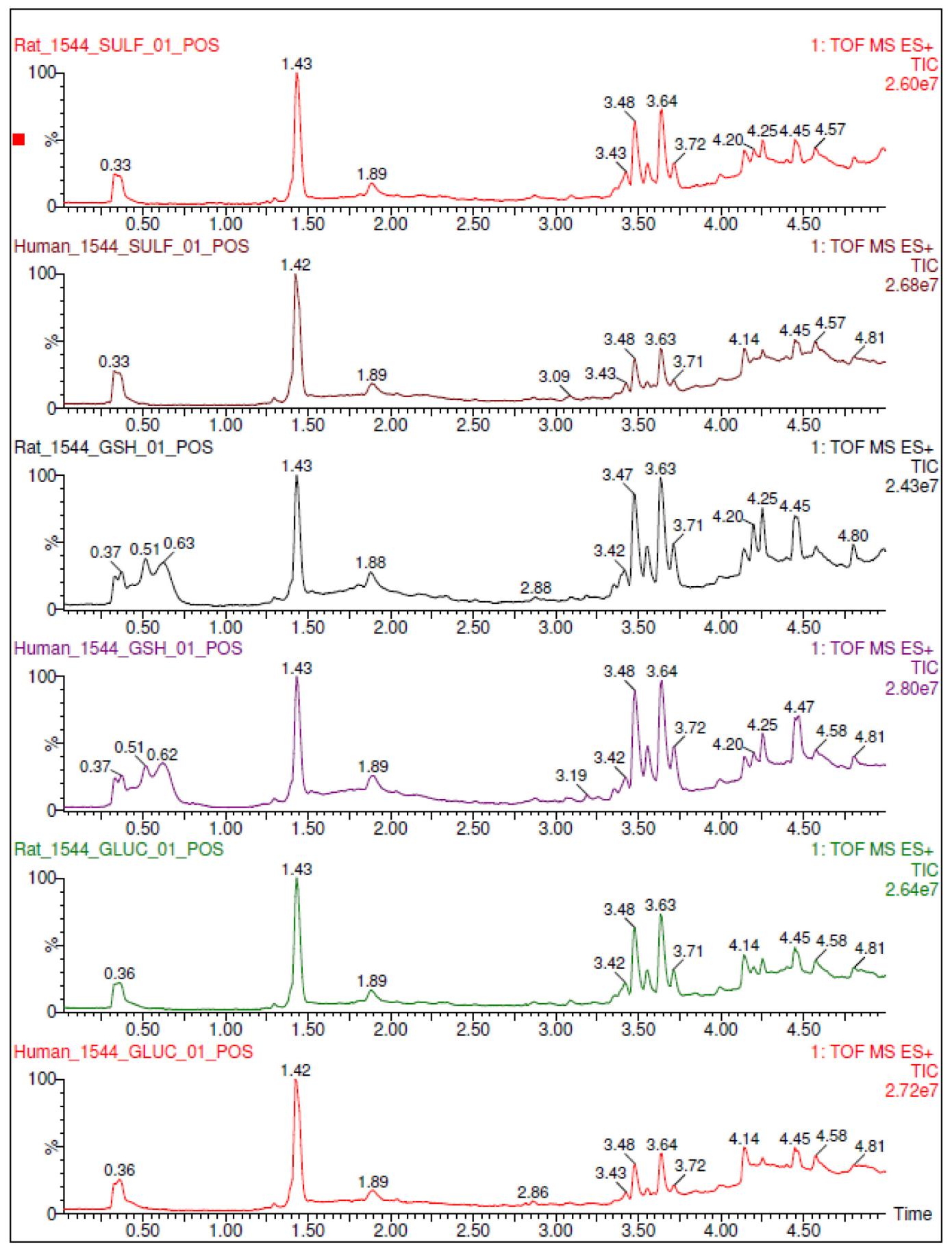

Figure 42. Comparison of Microsomal HRMS Extracted TIC for Lee 1544 


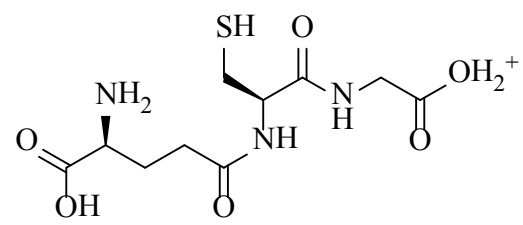

m/z: 308.0911 (100.0\%), 309.0945 (10.8\%), 310.0869 (4.5\%), 310.0954 (1.2\%), 309.0882 (1.1\%)

Figure 43. Structure of Protonated GSH

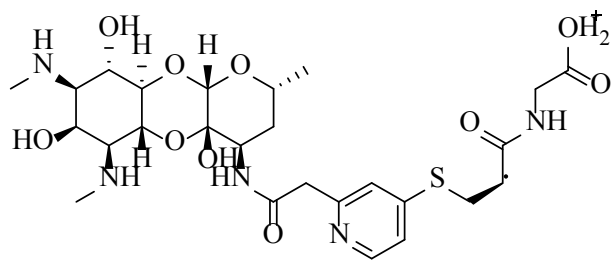

m/z: 613.2413 (100.0\%), $614.2446(28.1 \%), 615.2371$ (4.5\%), $615.2480(3.8 \%), 615.2455$ (2.1\%), $614.2383(1.8 \%), 616.2404$ $(1.3 \%)$

Figure 44. Structure of Protonated 1544 GSH Conjugation Product 
protein precipitation. Recent modifications in our methodology have allowed the detection of the 1544 parent out to the 60 hour timepoint. (Figure 45).

\section{HRMS Stability Analysis Conclusions and Discussion}

We have been able to identify free glutathione as well as a previously unknown 1544 GSH-conjugation product aided by the high sensitivity of the qTOF. Recent enhancements to chromatographic methodology have enabled us to track the 1544 parent in rat urine over two days after a single dose of investigational compound is administered. The sensitivity of the HRMS has not only enabled the discovery of new metabolites but has provided the ability to detect extremely small concentrations of analyte in complex matrices. In spite of this, I was unable to detect the 1544 GSH conjugation product identified from in-vitro analysis in the in-vivo studies. However, I do not believe this to be a fault of the analytical technique, as GSH conjugation products are known to be rapidly degraded in rodent surrogates.

Unfortunately, the instruments high sensitivity comes at a relatively high cost. In sensitivity mode the accurate mass information is simply not 'accurate' enough for effective mass defect tracking or the prediction of isotopic chemical formula. The relatively low single dose of drug complex with the incredibly complex background of urine in many ways limit the application of advanced MS techniques in the same way as the highly polar molecules limit the application of conventional chromatographic tools.

For this reason many leading PK laboratories employ a new generation of TQDMS instrumentation for the investigation of phase-II metabolic conjugation through neutral loss and precursor scanning, unfortunately the TQD we have available in our department simply does not have the sensitivity or dynamic range to facilitate such experimentation.

In this section I wish to discuss the general lessons learned from my graduate work which have largely focused around the use of mass spectroscopy to study drug metabolites and bacterial natural products. In generating this thesis I have been advised a dissertation is meant to contain a succinct work and does not have to contain everything you have accomplished in graduate school. While at St. Jude I have generated countless HRMS metabolic profiles for investigational (PD30, Nitazol, FL1) new agents and marketed drugs alike. Cetrorelix, a new compound, has been included in this work (Appendix C) to demonstrate the principles of PK profiling.. While I believe our HRMS PK techniques have seen considerable successes, in many ways our PK workflow is restricted by the lack of a high sensitivity TQD. As previously mentioned most PK laboratories now utilize the improved dynamic range and sensitivity of modern TDQ's to perform precursor and neutral loss scanning experiments for the characterization of low level metabolites. Unfortunately our current TQD simply does not have the sensitivity or dynamic range to facilitate the investigation of very dilute analytes. I believe our laboratories' efforts would receive great benefit through the acquisition of a more appropriate TQD-MS instrumentation. 


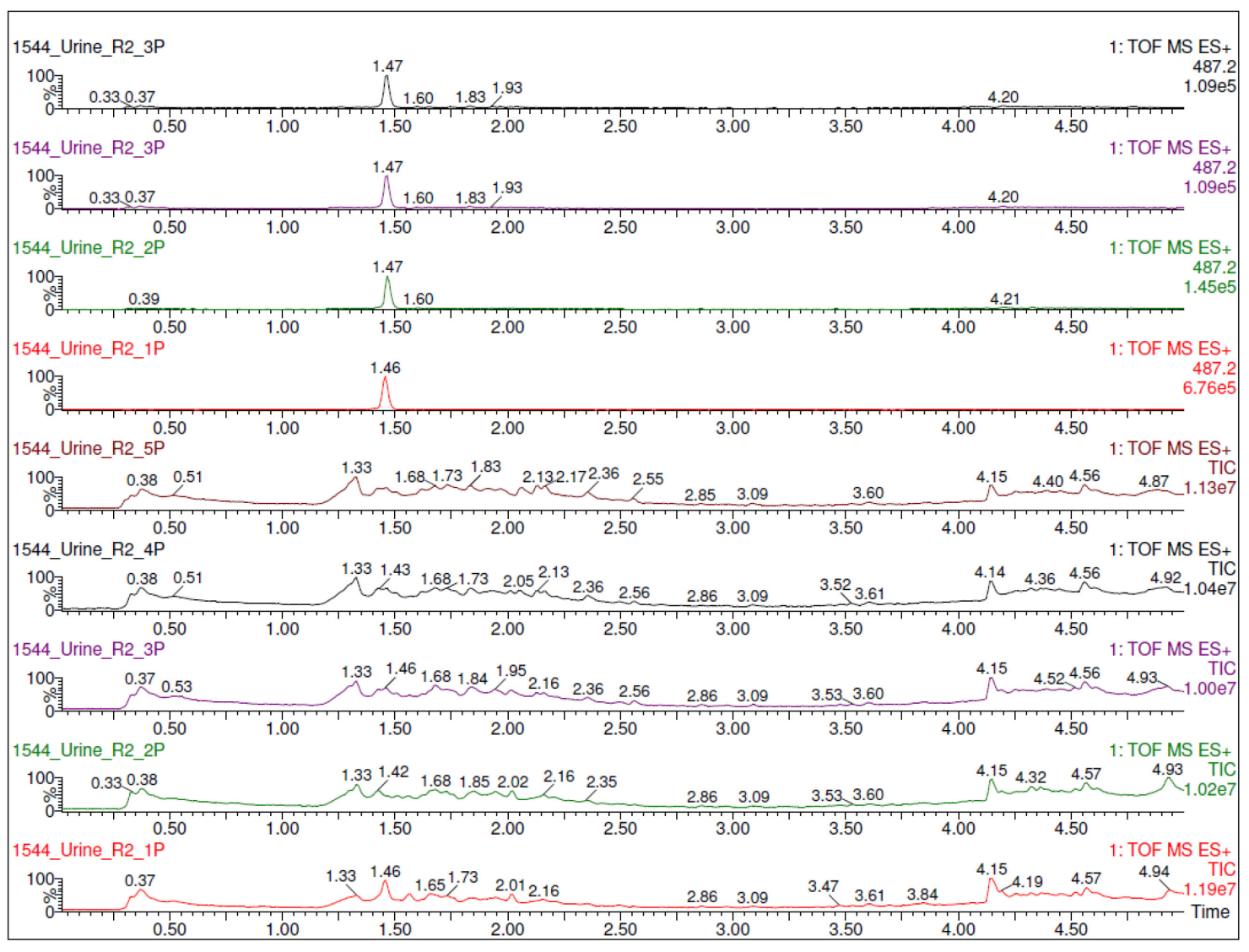

\section{Figure 45. 1544 Urinalysis TIC and Extract Ion Spectra}

NOTE: The parent 1544 can be easily traced past the sixty hour time point demonstrating the sensitivity of the HRMS technique. 
A number of instrument manufactures currently offer TQD-MS systems designed for the quantification of extremely dilute analytes. I believe the most appropriate instrument for the analysis of both dilute pharmacokinetic samples as well as complex bacterial natural products lies in an AB SCIEX 6500. This modern TQD would afford a near 20 fold increase in dynamic range and sensitivity compared to our current Waters TQD. Additionally, the 6500 is the first triple quadrupole to offer both ion mobility drive (SelexION) and a pulsed ion source in a single instrument. The application of ion mobility technology enables the interrogation of extremely complex samples (Ft lipid) while the pulsed ion source serves to significantly reduce background noise and further increase sensitivity.

Using a new generation of powerful mass spectrometers the field of preclinical pharmacokinetics (ADME) has witnessed intense growth over the past ten years, 163 with our hospital (SJCRH) recently forming a preclinical PK core for the optimization of internally synthesized chemotherapeutic agents. I believe the continued application of mass spectrometric techniques in this area will continue to offer a number of benefits both scientifically and financially.

To the scientist, early ADME data offer an opportunity to rapidly evaluate a potential therapeutic agent's metabolic stability, biological distribution and route of elimination as well as simple metrics such as plasma protein binding and solubility. The availability of this type of information at the laboratory or department level allows the individual chemist to rapidly evaluate synthetic molecule and propose new chemical iterations in a rapid succession. This information also serves to provide insights into the potential chemical reactivity of prostatic groups and the production of highly reactive intermediates (toxicity). While independently these types of analysis are in no way new, the ability to rapidly evaluate synthetic chemotherapies in-house potentially allows each scaffold enumeration to occur in a matter of weeks as opposed to the months or years required through more traditional lot based synthetic and analytical models.

From a financial standpoint, the in laboratory analysis of preclinical ADME allow unfavorable compounds to be excluded from the development pipeline much more rapidly than through traditional techniques. This pipeline annotation allows the scientist to evaluate the ADME implications of a particular scaffold or prosthetic modification prior to selecting the candidates for further enumeration This ability to further develop only the "most fit" compounds from a particular series not only yields dividends but helps to prohibit the submission of unfit compounds to the extremely costly and failure prone (previously outlined) NCE evaluation workflow.

\section{Introduction to Antitubercular Nitrofurans}

It is currently held RIPE TB therapy must be maintained for very long durations to effectively combat latent and/or slow-growing populations of bacteria. ${ }^{164}$ These subpopulations of $\mathrm{Tb}$ infection are believed to be innately resistant to current front-line antibiotics due to a reduction in bacterial metabolism. ${ }^{165}$ Furthermore, these latent 
populations often manifest as granulomas, further encapsulating the bacterium in an acidic, hypoxic (microaerophilic) environment, ${ }^{166}$ an environment in which nitroaromatic antibiotics, including clinical candidate PA824, are thought to be one of the few classes of drug able to act. Our laboratories have been developing an analogous series of nitrofuran isoxazolines designed to effectively target latent tuberculosis, which has also exhibited significant efficacy against oxygen starved (NRPII) tuberculosis in-vivo. ${ }^{167}$ Through the specific targeting of these metabolically hindered TB subpopulations new agents may soon be administered as an adjunct therapy to RIPE, thereby reducing the duration of TB chemotherapy and the potential for patient non-compliance.

\section{An Overview of Activity Based Profiling (Click)}

While the principals of activity tagging via cycloaddition of an analytical probe have been well established for the investigation and elucidation of large molecules; our laboratory sought to apply a derivative of the Cravatt technique to the investigation of small molecule metabolites in those of nitrofuran antibiotics. The following will briefly discuss the Cravatt Click methodology as applied to the investigation of a large molecule (serine hydrolase) for the purpose of introducing the highly validated workflows we now employ for the investigation of bacterial metabolites. It should also be noted the biotinstreptavatin analytical techniques first developed in concert with Jessani served as the foundation for the analytical processes utilized in the investigation of our "heavy" metabolic products.

Historically, proteomic techniques have sought to accurately measure the abundance of a target protein, and unfortunately, have only been able to provide an indirect metric of a proteins absolute activity. Similarly, many genomic tools have also been based upon the assumption that the abundance and activity of an enzyme remains directly proportional over time. Many believe this key linearity assumption

fundamentally limits the ultimate utility of conventional proteomic and genomic tools. ${ }^{168}$ In an attempt to overcome these limitations, Cravatt et al., sought to combine both chemical and biological tools into a multidisciplinary, Activity Based Protein Profiling (ABPP) approach "that utilizes active site-directed probes to profile the functional state of enzymes in whole proteomes."169

ABPP relies on the use of a synthetically derived chemical probe which interacts to label the active enzyme but disregards inactive or chemically inhibited isoforms, thereby directly reporting only the active fraction of a given protein complex. This metric of direct activity has proven greatly beneficial in the elucidation of major post translational processes directly responsible for the regulation of enzyme function. Since the introduction of this new approach a number of highly specific chemical probes have been synthesized for use in a highly diverse set of clinically relevant targets. ${ }^{170}$

In 2005, Jessani and Cravatt, reported the successful application of ABPP in the identification of enzymes upregulated in invasive human cancer cell lines of differing tumor types, including urokinase (previously recognized in tumorigenesis) and the novel, 
integral membrane serine hydrolase, KIAA1363. ${ }^{171}$ The experiment consisted of essentially two parts; the first involved the introduction of biotinylated-rhodaminefluorophphosphonate (FP) tags to prefractionated samples of human cancer cell line proteomes. The fractions were allowed to react at room temperature for one hour, and then treated with PNGaseF to deglycosylate the proteomes, reactions were quenched, run on SDS/PAGE and visualized via a fluorescence scanner. Absolute band intensities were calculated and integrated for the labeled proteins, enzyme activities were then tabulated by comparing multiple independently labeled reactions, data points were then averaged to report enzyme activities across each particular cell line. ${ }^{172}$

Secondly, following gel-based separation; the labeled analytes were isolated using standard biotin-streptavatin based solid phase affinity purification techniques. The enriched FP-labeled proteins were again separated via SDS/PAGE, with the resultant bands mechanically harvested and subjected to trypsin digestion. The peptidic fragments were then fixed using 2,5-dihydroxybenzoic acid (DHB) and analyzed using standard MALDI-TOF based analytical techniques, the resultant data was then cross-referenced against known FP-labeled and native proteins via public protein databases. ${ }^{173}$

In an attempt to provide a consolidated reporting of enzyme activities profiles, the author conducted a cluster analysis seeking to visualize the serine hydrolase concentrations averaged against a cell line known to be highly active, this relativized data was then subjected to a hierarchical clustering algorithm with the average linkage the determinate of similarity. As noted earlier, the novel membrane associated serine hydrolase KIAA1363 was found to be consistently upregulated and has been proposed by the authors to represent a novel marker for cancer cell invasiveness. In addition to the identification of KIAA1363 the authors go on to report a number of interesting pathways mediated via post translational modifications, namely in cathespin A and urokinase, which would have been near impossible to discern without the use of ABPP methodology. ${ }^{174}$

\section{Rational for Click Metabolic Pulldown}

To date, a Scifinder search of "Activity Based Protein Profiling" yields over 325 discrete hits. This volume of work conclusively illustrates the usefulness of ABPP in the characterization of human cancers, as well as demonstrates the potential utility of activity mediated cellular tagging. Following these ideas, and after careful consideration of the relevant literature, our lab sot to employ similar tagging and gel based separation techniques to investigate the method of action (MOA) and metabolic fate of nitrofuran based antimicrobial agents.

Nitroaromatic drugs, in particular nitroimidazoles PA824 and OPC67638 are showing great promise as antitubercular agents in current clinical trials ${ }^{175}$. For this class reductive metabolic activation is known to be required for action. In 2008, Singh et al ${ }^{176}$., reported on nitrous oxide (NO) formation (Figure 46) and release by PA-824 (a bicyclic nitroimidazole) which shares a similar structure-activity relationship (SAR) to our 


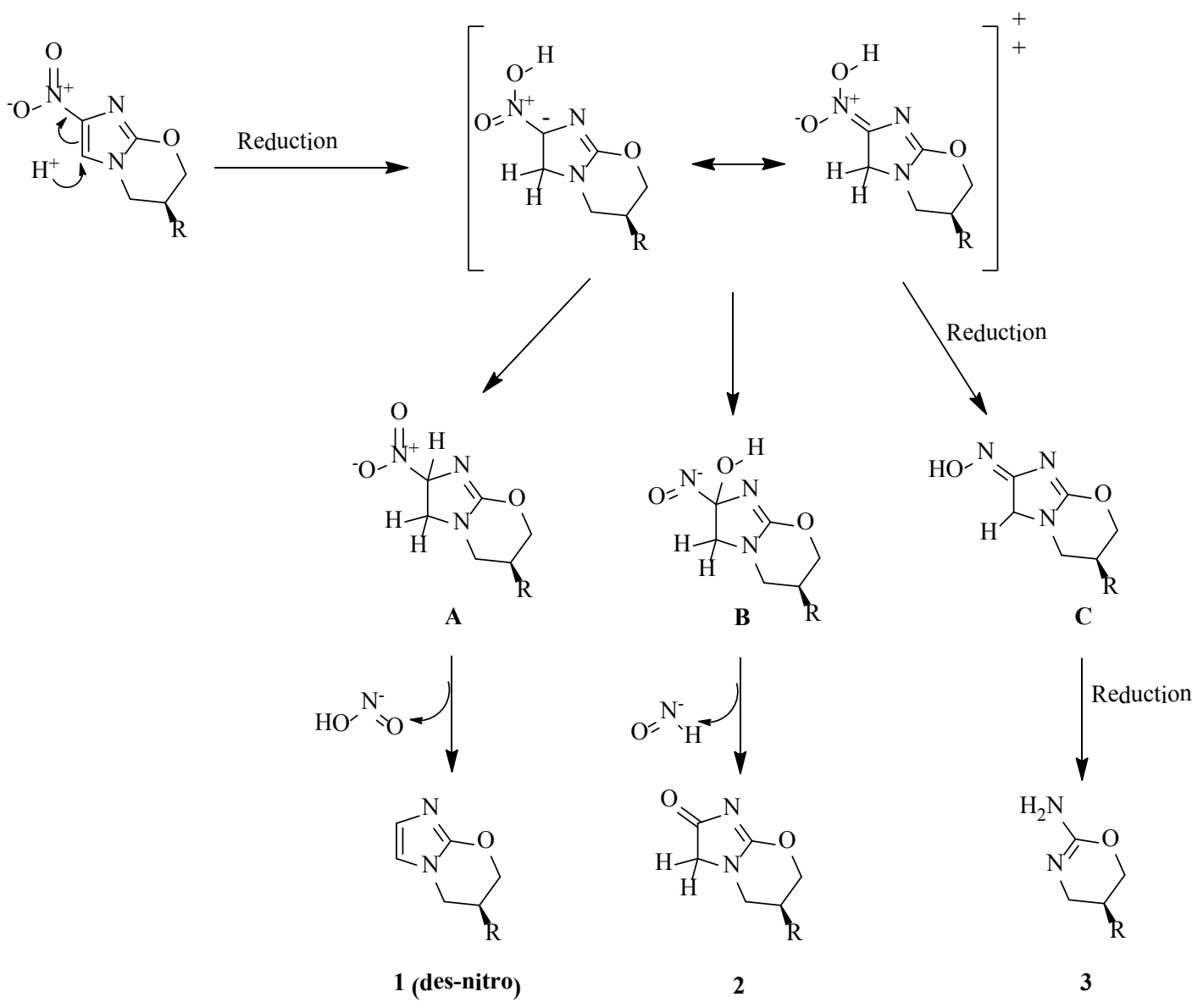

Figure 46. Proposed Imidazole Metabolic Pathway Illustrating the Generation and Release of Highly Activated NO Species 
nitrofuran analogs presumably as they reductively activated by similar pathways. However it is thought that the ultimate target of nitroimidazole and nitrofuran drugs is different, as the nitrofuran activated species is more stable and likely to form adducts. Coupled with the knowledge of ABPP principles, we set out to investigate the metabolic fate of our novel antimicrobial compounds as the nitrofuran metabolic process is as yet unknown in M. tuberculosis.

The structure of traditional nitrofuran antimicrobial agents is illustrated for reference in Figure 47 (Nitrofurantoin) and representative antitubercular nitrofuran developed by our laboratory (Figure 48). In preliminary studies we observed that our nitrofurans did not release NO as observed in the Singh study with PA824 (Figure 49) which lead us to hypothesize that they act through radical addition rather than NO generation. To support this hypothesis we sought an indirect approach to study nitrofuran activation and targeting, in much the same way as Cravatt, has sought to supplement indirect proteomic click tagging techniques.

Having theorized nitrofuran compounds might undergo a metabolic fate different to that of Singh's nitroimidazoles, and drawing inspiration from another method often championed by the Cravatt lab, we designed an experiment based upon the incorporation of a so called "click" tag170 (Figure 50). This cycloaddition technique, popularized by Sharpless, 177 is somewhat unique in its' ability to couple an azide to alkyne in highly aqueous environments. To this end, we synthesized a number of our nitrofuran antimicrobial compounds to contain a solvent accessible alkyne. The probe molecules were designed in a way as to position the cycloaddition site away from bounds though necessary for protein-ligand interactions. All cycloaddition probes were tested for preservation of MIC, the six most active compounds were selected for continued investigation (Figure 51 and Table 1). We believed the incorporation of these high affinity tags would allow the investigation of targets of nitrofuran metabolic activation and ultimately mycobacterial inhibition. For simplicities sake, we termed the two potential targets the "light" pathway and the "heavy" pathway based on the molecular weight of the products. In the light fraction, small molecule conjugates and activated nitrofuran decomposition products were selected for via the click inclusion of a high activity fluorescence tag to allow detection of the product conjugates at low levels. In particular, one proposed conjugation was mycothiol, which is the mycobacterial analog of glutathione, which function is thought to as a buffer in the mycobacterial cell against electrophilic species.

In the heavy fractions we sought to recover probe-protein conjugates, to see if there was a dominant proteinacous target for this inhibitor class. In both cases the small and biological alkyne "click" tag should allow the probes to interact with the nitrofuran activating enzymes and find their ultimate cellular target without blocking the binding state of the drugs as would be the case with larger covalently tagged probes. The click tags can then be conjugated to a fluorophore for the light fraction or biotin for the heavy fraction to allow streptavidin capture and subsequent protein analysis. From this point the methodology was straight forward; the probe was incubated in the presence of M. Bovis 


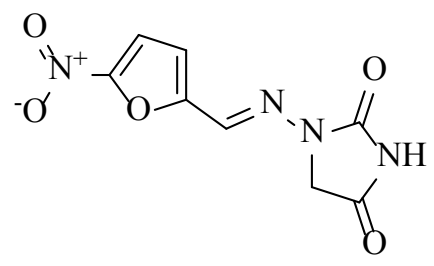

Figure 47. Structure of Nitrofurantoin

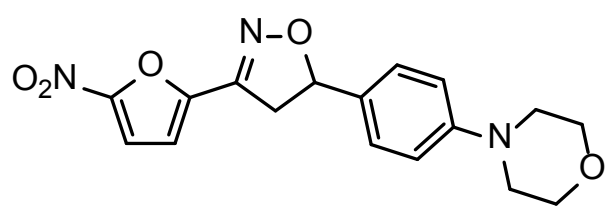

Figure 48. Structure of Lee 1055

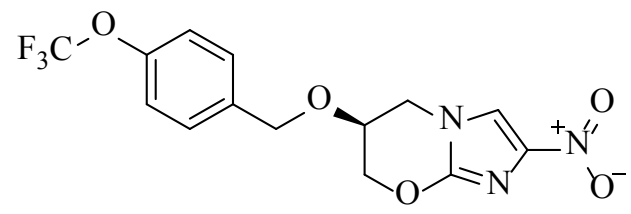

Figure 49. Structure of PA-824 


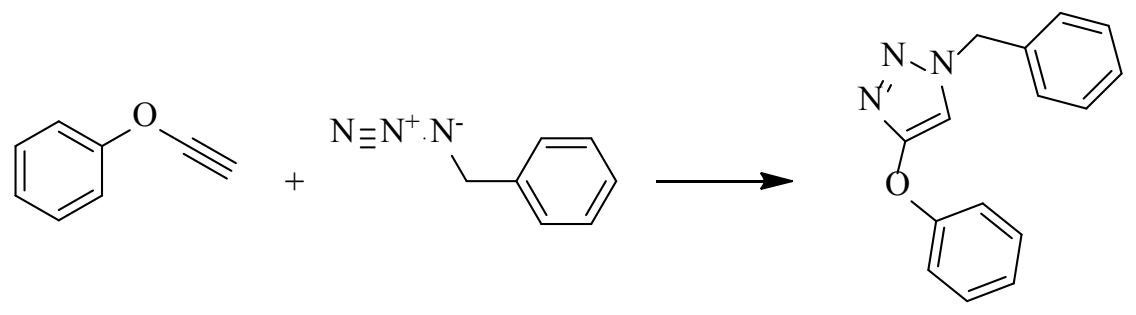

Figure 50. Generalized 1,3-Dipolar Azide-Alkyne Cycloaddition "Click"

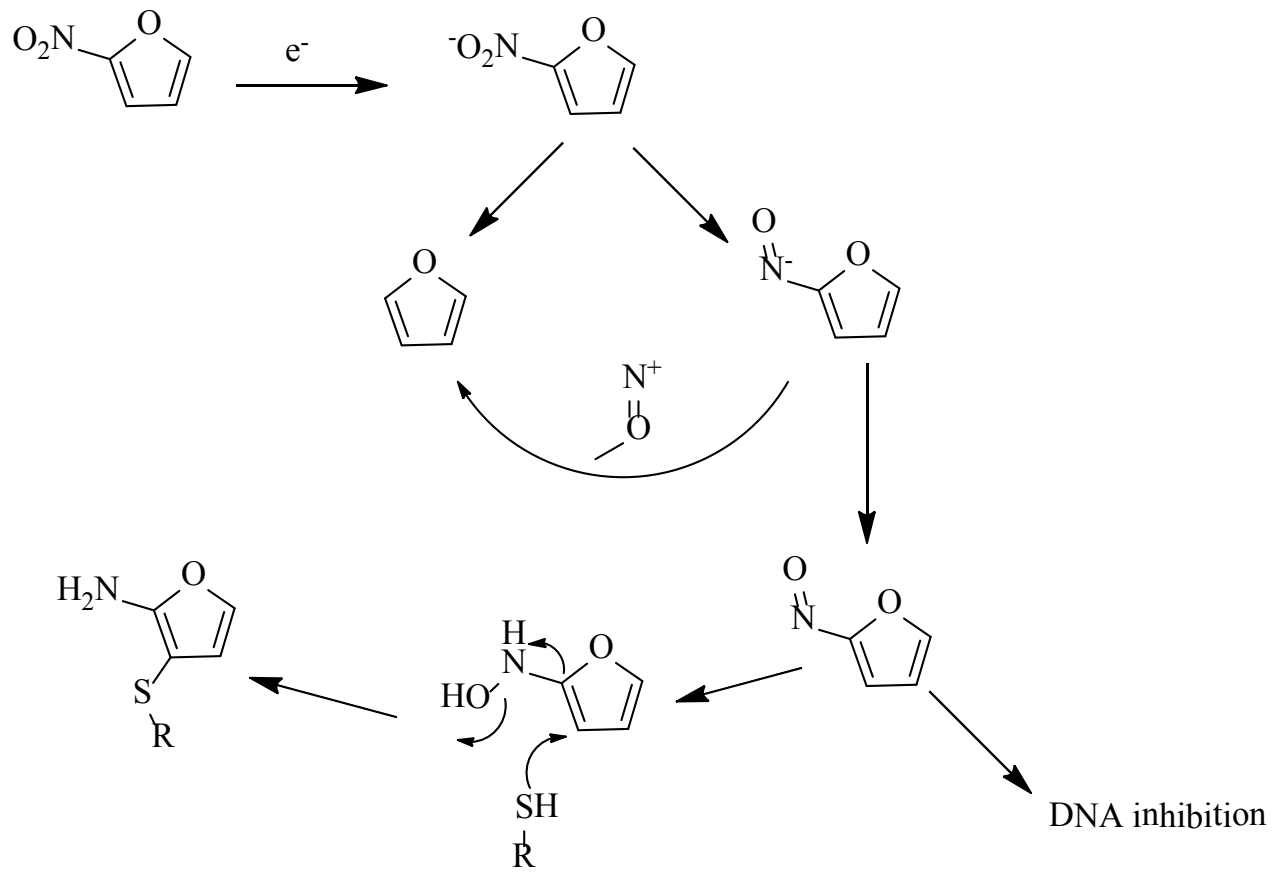

Figure 51. Proposed Nitrofuran Metabolic Pathway Illustrating the Electron Cascade Ultimately Resulting in DNA Intercalation or Mycothiol Conjugation 
Table 1. MICs of Acetylene Tagged Nitrofurans against Gram (+VE) Bacteria

\begin{tabular}{cccccccc}
\hline Click Probe & $\begin{array}{c}\text { B. } \\
\text { anthracis } \\
\text { sterne }\end{array}$ & E. faecalis & $\begin{array}{c}\text { S. aureus } \\
\text { ATCC29213 } \\
\text { (MSSA) }\end{array}$ & $\begin{array}{c}\text { S. } \\
\text { pyogenes }\end{array}$ & $\begin{array}{c}\text { S. } \\
\text { pneumoniae }\end{array}$ & $\begin{array}{c}\text { B. } \\
\text { subtilis }\end{array}$ & $\begin{array}{c}\text { MRSA } \\
\text { NRS70 }\end{array}$ \\
\hline Lee 1397 & $>200$ & 50 & 6.25 & 25 & $>200$ & 12.5 & 12.5 \\
Lee 1521 & $>200$ & 100 & 3.13 & 12.5 & 50 & 3.13 & 3.13 \\
Lee 1534 & $>200$ & 200 & 12.5 & 50 & 100 & 25 & 6.25 \\
Lee 1575 & $>200$ & 200 & 12.5 & 50 & 100 & 12.5 & 3.13 \\
Lee 1595 & $>200$ & 200 & 6.25 & 12.5 & 50 & 6.25 & 6.25 \\
Lee 1618 & $>200$ & $>200$ & 100 & $>200$ & 100 & 200 & $>200$ \\
\hline
\end{tabular}


(BCG) for 30 to 120 minutes, the cells were harvested and then washed to remove any unreacted probe. The cells were then mechanically lysed and separated into two fractions based upon molecular weight through the use of centrifugal 2,000 Daltonmolecular weight cut-off filters. These heavy and light fractions were then reacted or "clicked" to one of two reporters; a fluorescent tag for the LC analysis of the light fractions (Figure 52) or a biotinylated linker to facilitate affinity separation of the proteins contained in the heavy fractions. (Figure 53).

The unreacted florescent azide elutes very near the conjugation product. To evaluate the chemical cycloaddition process a click probe (Lee-1595 is illustrated in the fluorescence spectra below) was allowed to react with the fluorescent azide in PBS. The unreacted fluorophore elutes last (TR 17.933) slightly following the elution of the cycloaddition product (TR 17.525). While the two compounds were closely eluting even on a longer (BPD) method, fractions were successfully collected and their identities conformed via MS. (Figure 54) Analytical HPLC fluorescence spectra of 1595 conjugation products evaluating the use of $\mathrm{MeOH}$ (Figure 55) and $\mathrm{ACN}$ (Figure 56) organic mobile phases, $\mathrm{MeOH}$ was chosen due to the increases in absolute intensity, despite its slightly poorer chromatographic properties.

Figure 57 illustrates the results of the click conjugation reaction product cycloaddition proceeded to near 100\% completion. Figure 58 illustrates the fluorescence spectra from the cycloaddition reaction in BCG extract. In this spectra below, 1 the parent (drug + fluorophore) conjugation product can be identified through the use of retention times (TR 6.93). A number of fluorescently tagged, presumably more polar (more early eluting) molecules can be seen with significant fluorescent peaks appearing at $T_{R} 6.08$, $6.38,6.61$, respectively. As these fluorescent peaks do not elute with either the unbound fluorophore or the fluorescently tagged drug conjugate, they must be fluorescently tagged molecules of an unknown nature. An effort was made to collect fractions but the short half-life of the potential metabolic products and dilute nature of the fractions proved to greatly complicate MS detection.

The "light" products were then separated and analyzed via reverse phase fluorescence HPLC. The heavy products were "pulled down" using solid phase supported streptavatin beads and run out on SDS/PAGE gels. The gels were then robotically harvested, digested and analyzed via nanospray LC/MS, the resultant data was submitted for database searching. We are currently in the process of analyzing the "heavy fraction" search results in hopes of identifying the ultimate target of our nitrofuran based compounds. A number of LC runs seem to confirm the presence of low level fluorescent metabolites in the click tagged (light) bacterial fractions. We are now faced with the task of identifying these short-lived compounds; we have attempted the "semi-analytical" HPLC fractionation of the fluorescence peaks, then UPLC/MS of the resultant fractions. This offline method has not proven successful; the compounds seem to degrade between the purification and MS steps, while we currently do not have an UPLC/MS with a fluorescence detector, we are in the process of correcting this potential shortcoming. 

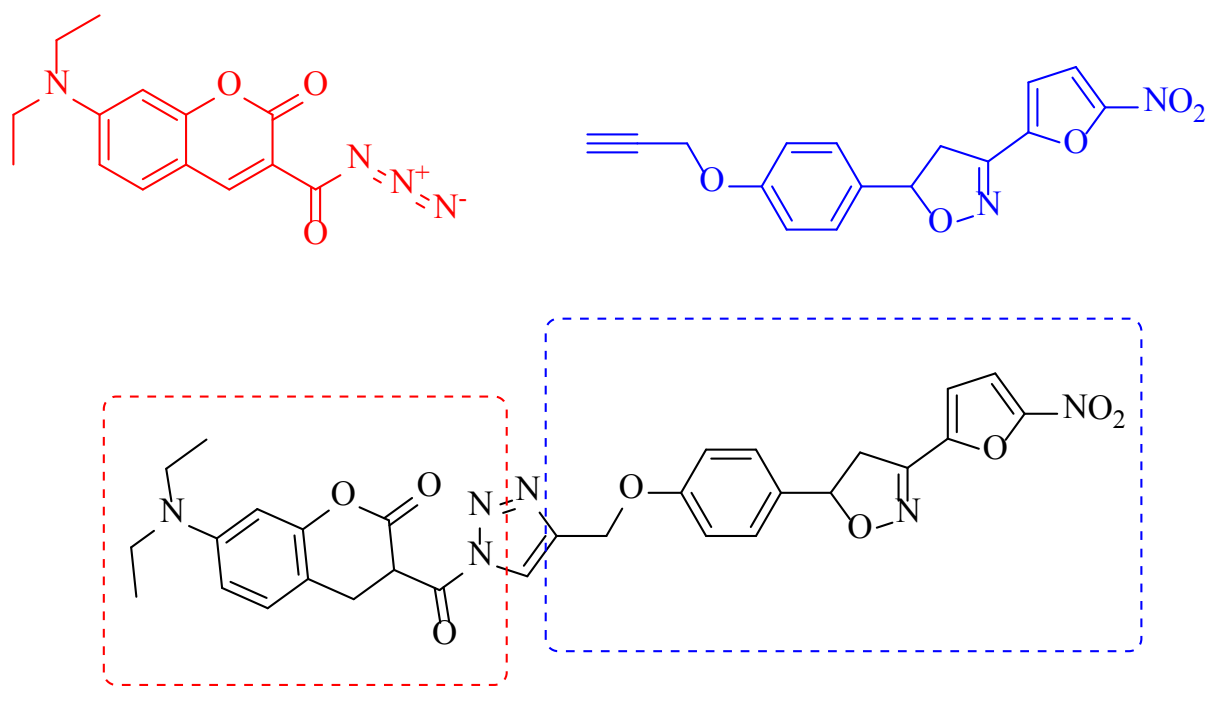

Figure 52. Click Conjugation of a Light Fluorescent Probe Resulting in the Formation of the Fluor-Drug Cycloaddition Product 

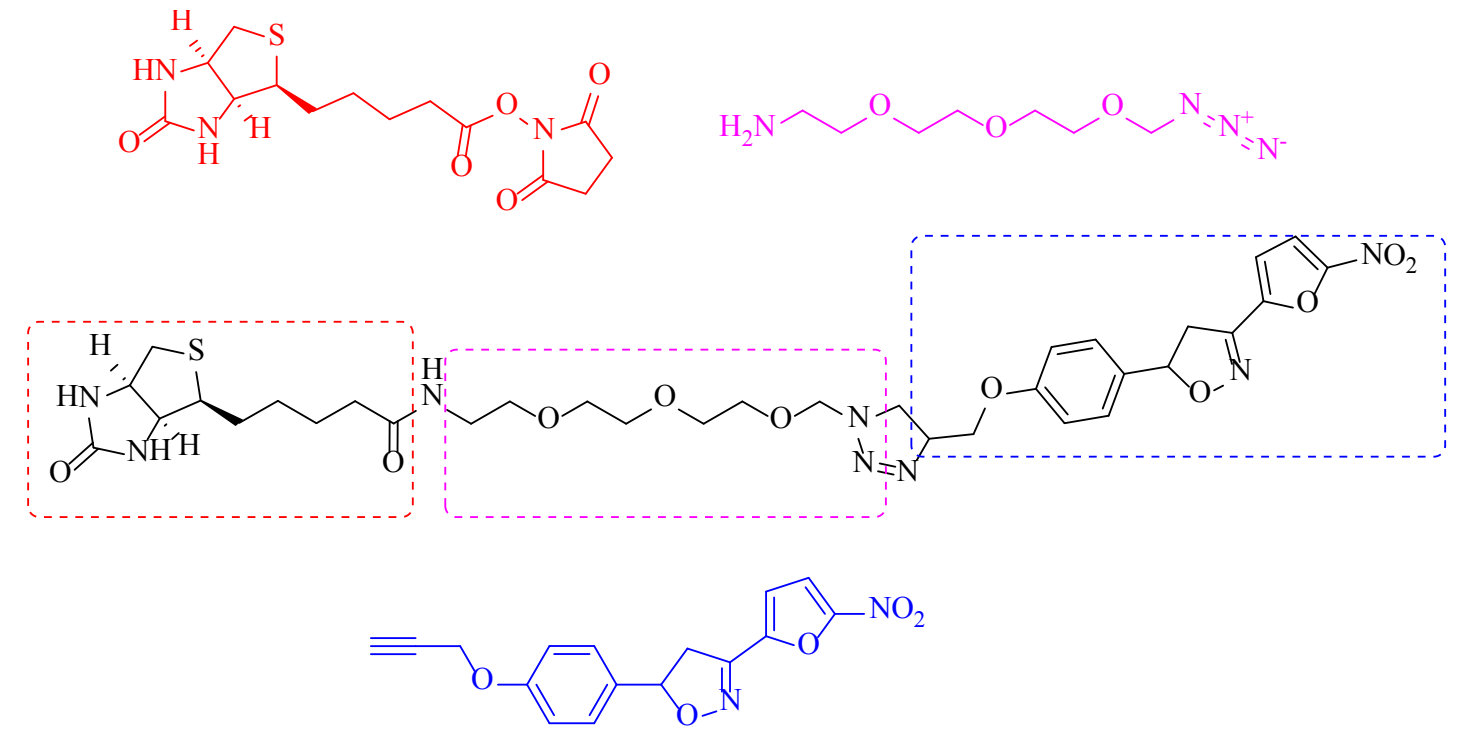

Figure 53. Click Conjugation of a Heavy Biotinylated Probe Resulting in the Formation of the Biotin-Linker-Drug Cycloaddition Product 


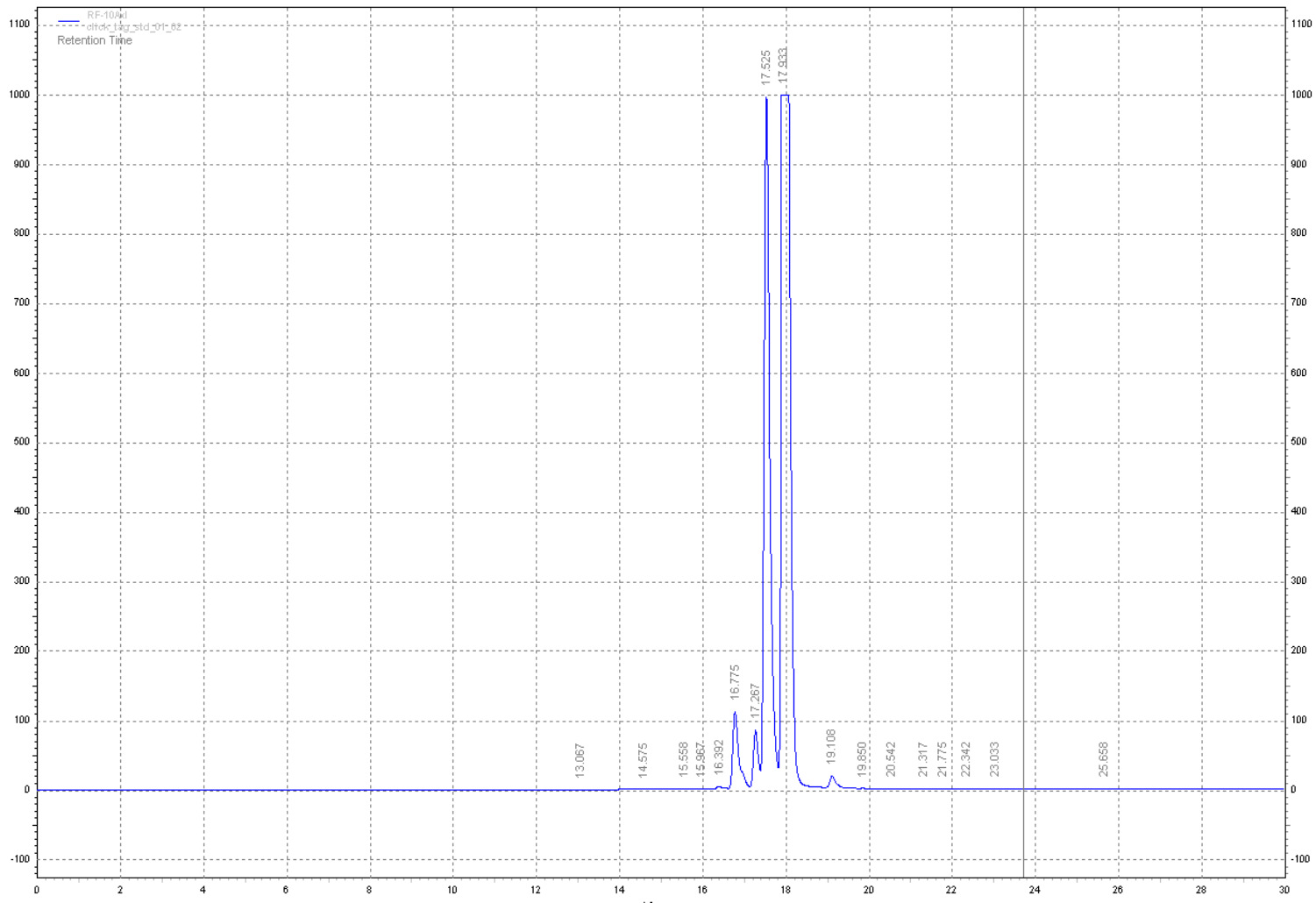

Figure 54. Fluorescence Spectra of Semi-Analytical HPLC Separation of Click Products 


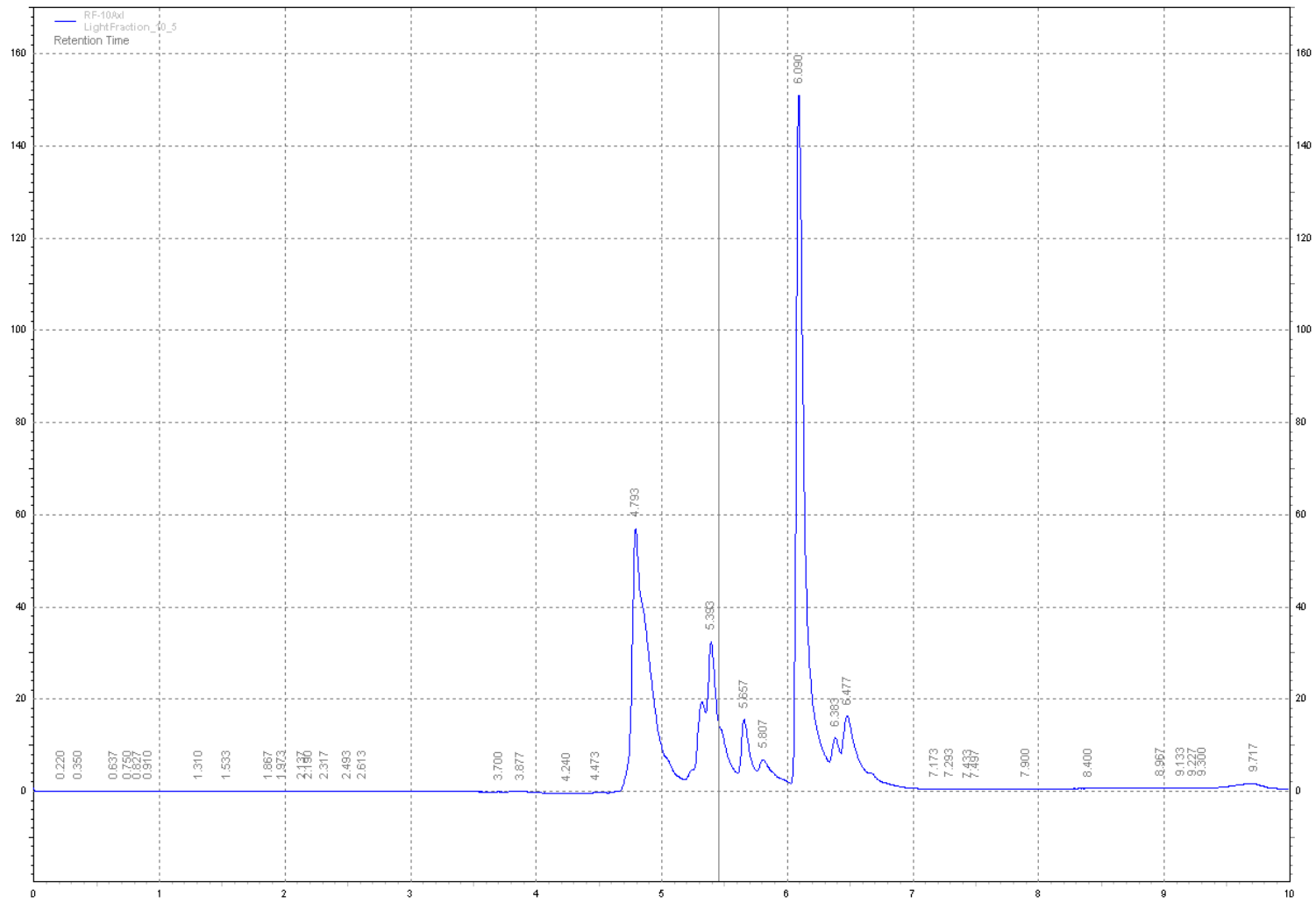

Figure 55. Analytical HPLC Separation of 1595 Conjugation Products with MeOH Organic Mobile Phase 


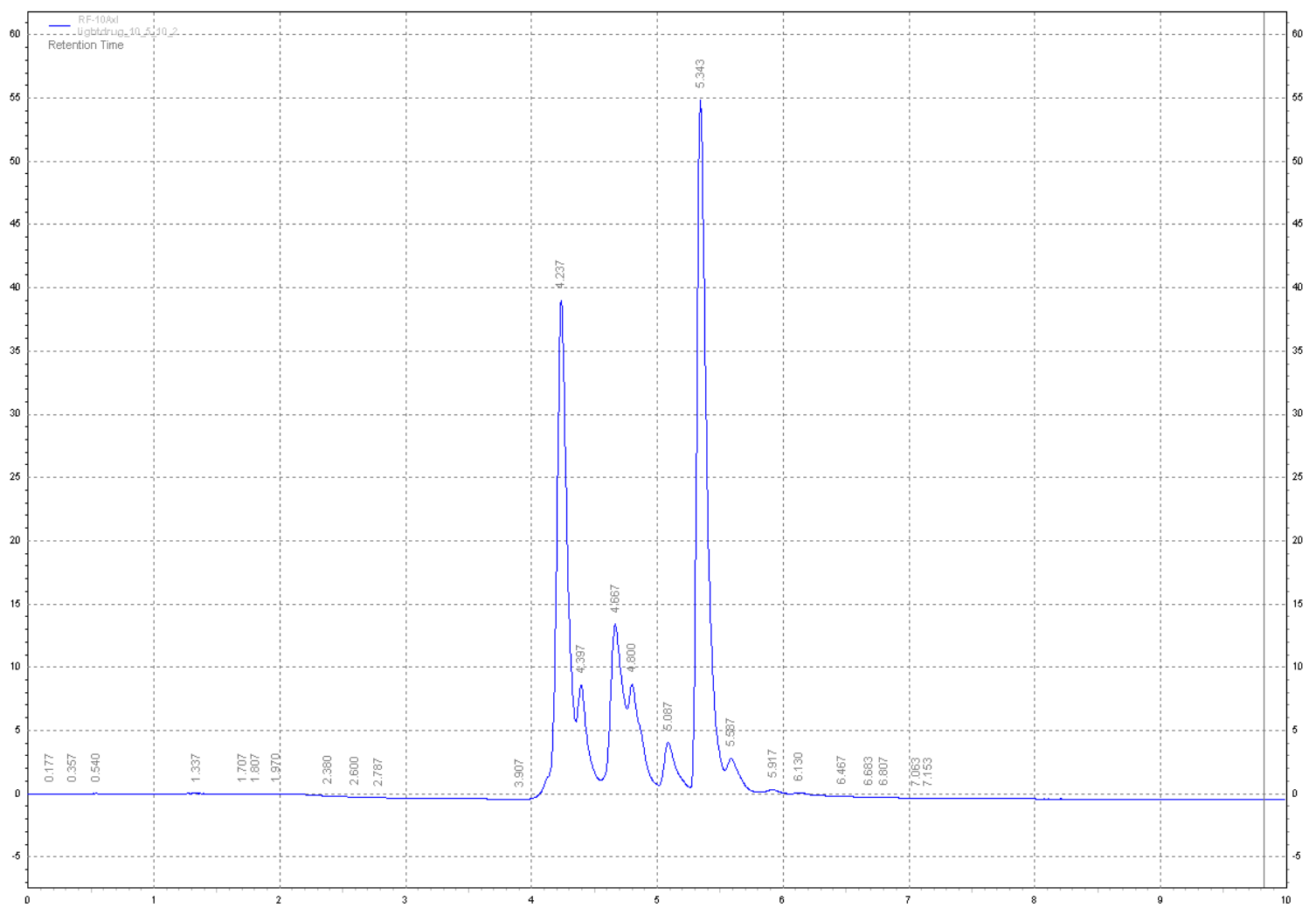

Figure 56. Analytical HPLC Separation of 1595 Conjugation Products with ACN Organic Mobile Phase 


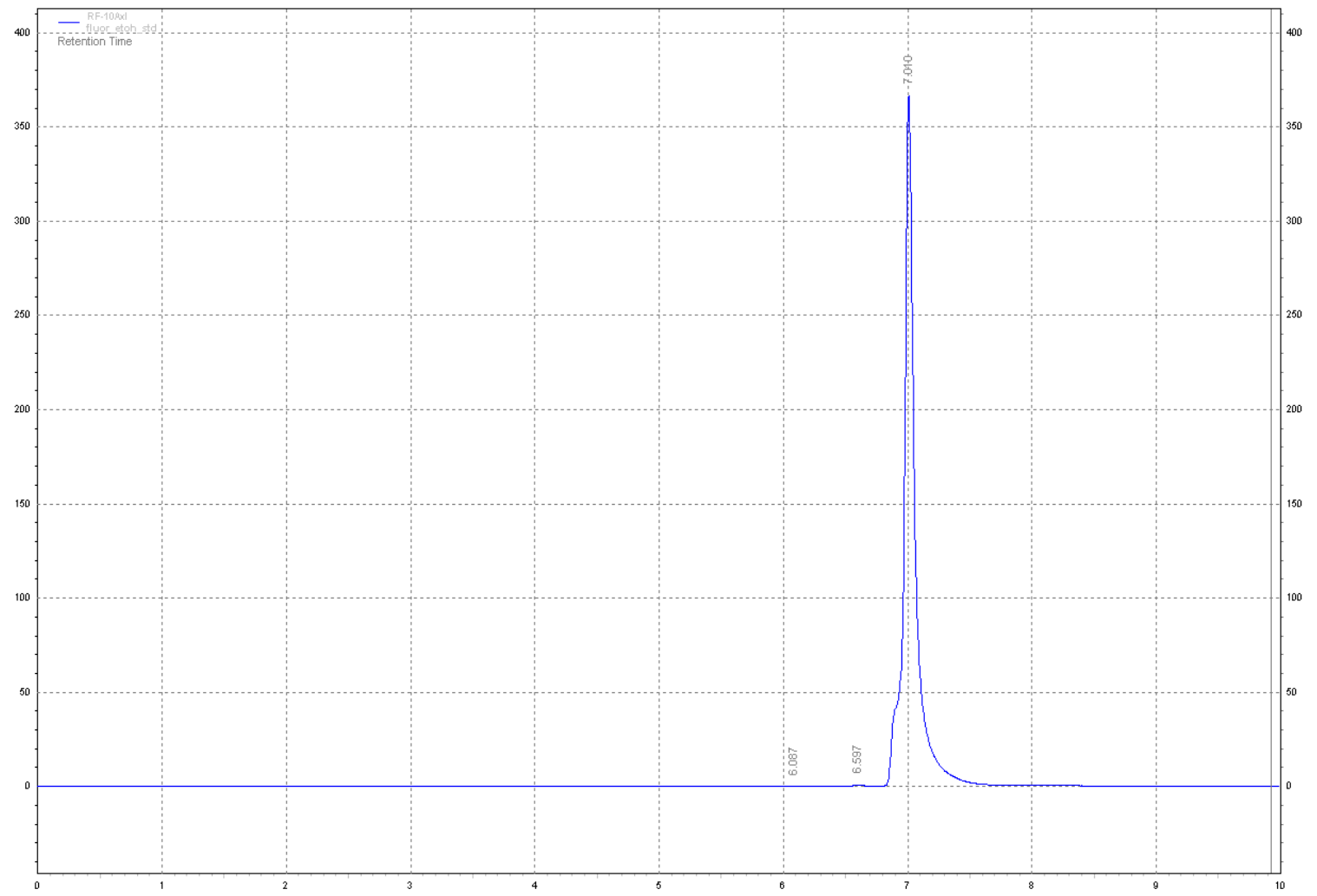

Figure 57. Analytical HPLC Analytical Fluorescence Spectra of 1521

Fluorometric Conjugation Product in PBS + Catalytic TBTA 


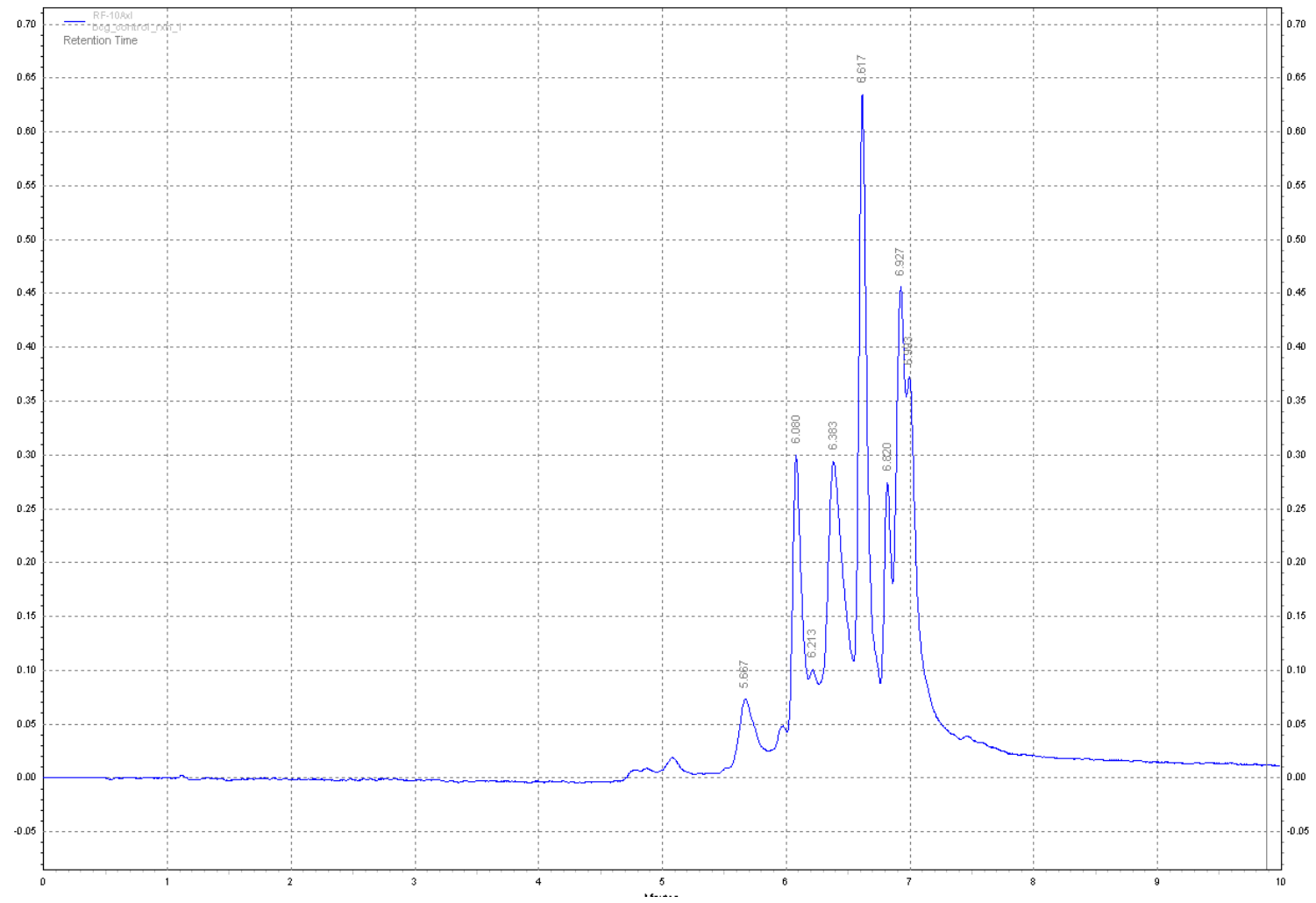

Figure 58. Analytical HPLC Fluorescence Spectra of 1521 following BCG Incubation and Click Tagging

NOTE: Numerous presumably metabolic products can be visualized; however, the very dilute analytes made further offline MS analysis impossible. 
Our laboratory has currently synthesized six nitrofuran based "click" tags for use in the next iteration of this experiment. (Figures 59-64) Overall the MIC's of the click tagged compounds are quite similar to their non-derivatized parents (Table 2).

\section{Click Pulldown Conclusions and Discussion}

The click chemistry portion of this experiment proved to be quite successful despite initially suffering from low yields. The addition of catalytic amounts of tris[(1benzyl-1H-1,2,3-triazol-4-yl)methyl]amine (TBTA) (Figure 65) was found to greatly increase click yields through the stabilization of the copper/azide intermediate.178 The probes also retained reasonable antimicrobial activity upon addition of the acetylene tag. The shortcoming lied in that when our laboratory initially conducted these experiments we did not yet have access to a high sensitivity mass spectrometer.

The initial analytical workflow relied on the use of an HPLC equipped with only a UV/Vis and fluorescence detector. The hope was that the resultant fluorescent signals would be sufficient to collect small fractions of effluent using the fluorescence signal as a gate. While we were able to detect fluorescent components in the supernatant samples, we were unable to identify them any further, due to both the metabolites relatively short half-life and the incredible dilution factor involved in the collection of fractions.

The high mass click tagging system proved to be successful, it just failed to identify any novel proteins or suggest alternative modifications to previously known targets. However, I still remain quite confident in the utility of this analytical technique, as both tagging strategies were successful on their own right. The light fractionation project ultimately failed due to lack of instrumentation not experimental design. The high mass tagging worked; it just failed to find anything new.

In light of recent additions, to both departmental instrumentation, and due to an increased knowledge of the nitrofuran bacterial targets we are currently preparing to revisit the light click tagging procedure for the identification of both nitrofuran and other metabolites.

While this project has perhaps been the most challenging to date, I also believe it holds the most future promise as a tool uniquely suited to elucidate previously unknown

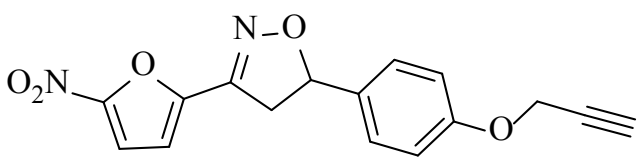

Figure 59. Structure of Click Probe Lee 1397 


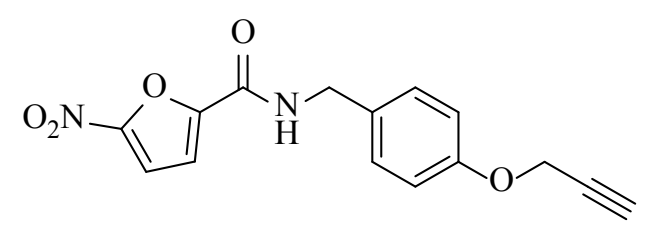

Figure 60. Structure of Click Probe Lee 1521

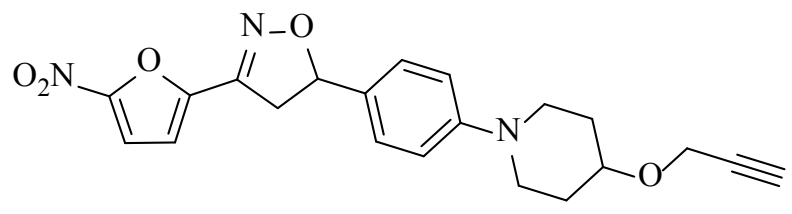

Figure 61. Structure of Click Probe Lee 1534

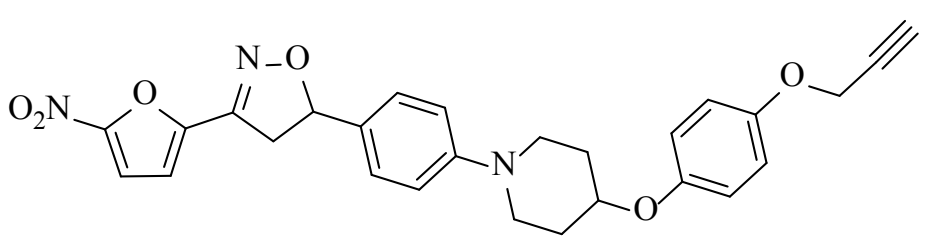

Figure 62. Structure of Click Probe Lee 1575

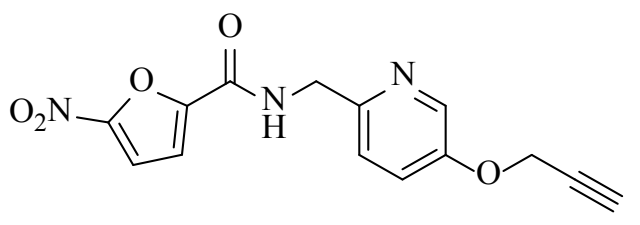

Figure 63. Structure of Click Probe Lee 1595 


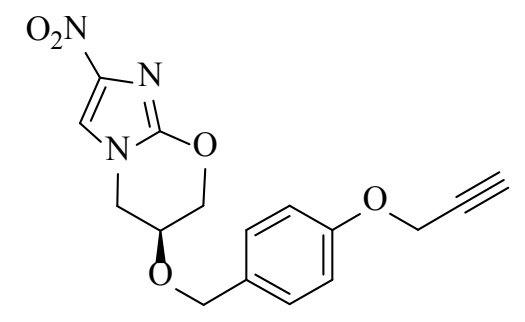

Figure 64. Structure of Click Probe Lee 1618 
Table 2. In-vitro ADME and MIC of Click Probes

\begin{tabular}{|c|c|c|c|c|c|c|c|}
\hline & $\begin{array}{c}\mathrm{Mtb} \\
\mathrm{MIC}\end{array}$ & $\begin{array}{c}\text { Solubility } \\
\mathrm{pH} 7.4) \\
\mathrm{ug} / \mathrm{mL}\end{array}$ & $\begin{array}{c}\text { Permeability } \\
\left(10^{-6} \mathrm{~cm} / \mathrm{s}\right)\end{array}$ & $\begin{array}{c}\mathrm{t} 1 / 2 \\
\text { (human) }\end{array}$ & $\begin{array}{c}\text { t1/2 } \\
\text { (mouse) }\end{array}$ & $\begin{array}{c}\text { Protein } \\
\text { binding } \\
\text { (human) }\end{array}$ & $\begin{array}{c}\text { Protein } \\
\text { binding } \\
\text { (mouse) }\end{array}$ \\
\hline Structure & 0.2 & 22.6 & 177.9 & 1.65 & 0.34 & 90.4 \\
\hline
\end{tabular}




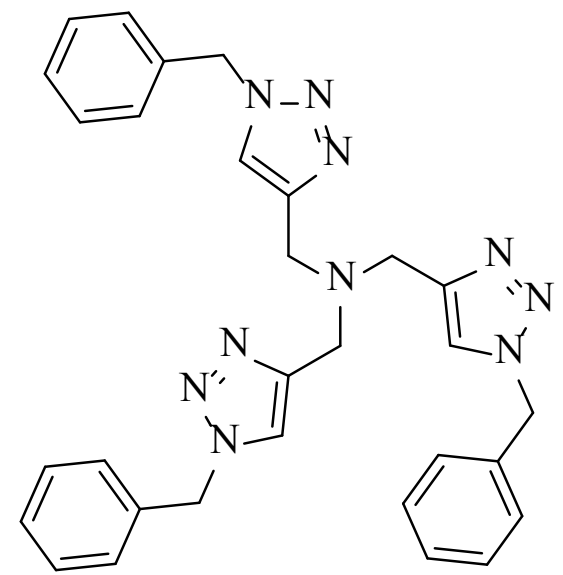

Figure 65. Structure of TBTA 
small molecule bacterial metabolic pathways for bioactivated drugs. We have shown a high yielding cycloaddition can be performed in a variety of solutions through the addition of catalytic amounts of TBTA. We have also been able to visualize fluorescent conjugations products upon incubation with BCG. Unfortunately, our limited analytical instrumentation coupled with the relatively unstable nature of the metabolites prevented the acquisition of fluorescence associated mass spectrometric values.

To address this shortcoming, I am currently in the process of coupling an ultrahigh resolution fluorescence detector (Shimadzu RF-10AXL) to our newly acquired UPLC-qTOF. Through the use of this specially hyphenated instrument I am confident we will finally achieve fluorescence and mass spectrometric data simultaneously. These two highly sensitive analytical techniques applied in concert will allow the identification and ultimate structural elucidation of the "light" click fragments which has eluded us to date. Upon significant validation, I believe these Click-HRMS techniques will serve to revolutionize the way not only our laboratory accesses scaffold stability, but serve to advance the field of antimicrobial research through the elucidation of previously unknown bacterial xenobiotic detoxification strategies.

The most immediate ways I would like to further this project include repeating the BCG nitrofuran experiment with our new instrument as well as further investigating the metabolism of our click probes in MRSA. In primary screening our click tagged nitrofuranal compounds displayed significant efficacy against MRSA (Table 3). As we are very near establishing a pipeline for the click pulldown and analysis of metabolic products it would be quite simple to "change bugs" in our experimental procedure. I believe these insights could prove significant in a number of ways, first as a cross validation of our analytical processes. Secondly, I would like to determine if nitrofuran metabolism differs between bacterial species? This line of analysis could also serve to evaluate the target of mycolactone as very little is currently known regarding the necrotic processes at work in Buruli ulcer. Once current handling and archival difficulties are addressed, as I believe they soon will be, I would like to further investigate mycolactone induced necrosis through the development of acetylene tagged mycolactone (Figures 66 and 67). The processes underlying the host response and detoxification of mycolactone are currently unknown.

I would also like to investigate the metabolic processes involved in the bacterial detoxification of linezolid, an oxazolidinone antibacterial agent proven to be effective in the treatment of vancomycin-resistant enterococci (VRE) as well as MRSA. In many ways linezolid constitutes our last line of defense against clinical presentations of gram positive vancomycin resistant bacteria associated skin and soft tissue infections (SSTI). While the approval of linezolid was heralded as a significant breakthrough in the battle against drug resistant bacterium, numerous clinical presentations of linezolid resistant infections have been reported. ${ }^{179}$ From the synthetic standpoint an acetylene tagged linezolid should be relatively easy to synthesize. This novel, click conjugated probe (Figure 68) could then prove instrumental to the interrogation of the linezolid bacterial bioremediation process and afford new insights into the metabolic mechanisms at work in linezolid resistant enterococci and staphylococcus. 


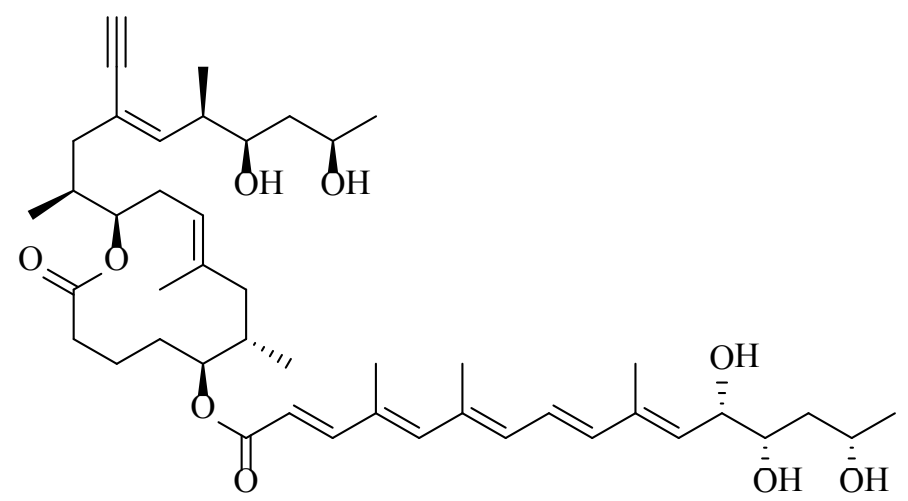

Figure 66. Structure of Proposed Mycolactone Click Probe to Enable HRMS Analysis of Polyketide Core

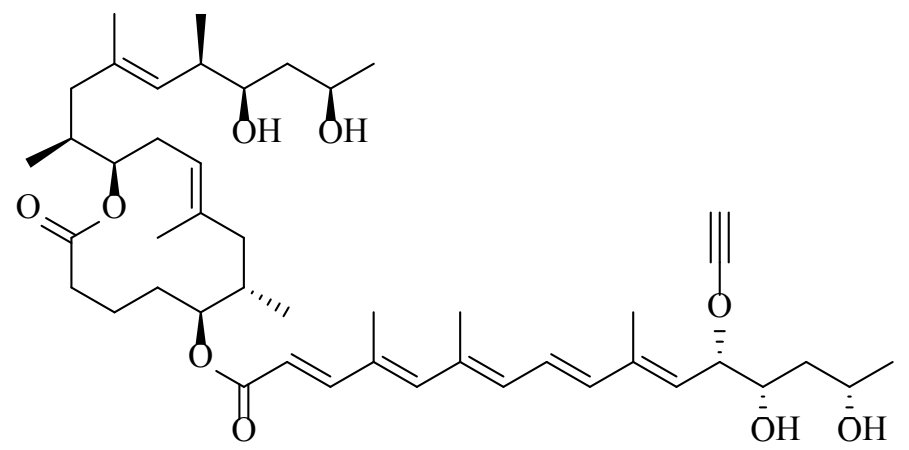

Figure 67. Structure of Proposed Mycolactone Click Probe to Enable HRMS Analysis of Acyl Side Chain

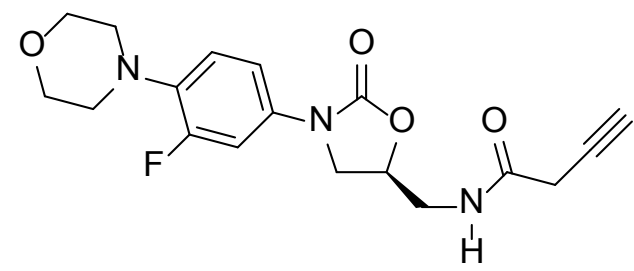

Figure 68. Structure of Proposed Linezolid Click Probe 


\section{Experimental Methods}

The experimental methods developed throughout this chapter are outlined below.

\section{HRMS Stability Analysis Methods}

\section{Preparation of Urinalysis Samples}

All urine samples were thawed at room temperature and briefly vortexed to homogenize. $50 \mu \mathrm{L}$ of each urine sample were transferred to microcentrifuge tubes containing $200 \mu \mathrm{L}$ of $\mathrm{CH}_{3} \mathrm{OH}$ and proteins allowed to precipitate at $-20^{\circ} \mathrm{C}$ for 20 minutes. The suspensions were clarified via ultracentrifugation and $80 \mu \mathrm{L}$ of the supernatant was removed for UPLC/MS analysis.

\section{UPLC/MS Analysis}

LC/MS analyses were performed on both a Waters Acquity UPLC-TQD and a Waters Acquity UPLC-qTOF. All dry analytes were solvated in $\mathrm{CH}_{3} \mathrm{OH}$ and filtered prior to analysis; liquid analytes were diluted in the same and filtered. Separations were achieved at $0.5 \mathrm{~mL} / \mathrm{min}$ via a 5 minute gradient elution from $95 \% \mathrm{H}_{2} \mathrm{O}+0.1 \%$ Formic Acid to $95 \%$ ACN $+0.1 \%$ Formic Acid as follows. The first 30 seconds were held at the starting conditions then ramped to $40 \%$ organic over two minutes followed by a ramp to $95 \%$ organic an additional two minutes then followed by a 30 second stop at the $95 \%$ organic before returning to re-equilibrate. A Waters Acquity C18 BEH $1.7 \mu$ x $50 \mathrm{x}$ $3.0 \mathrm{~mm}$ column was maintained at $40^{\circ} \mathrm{C}$ through-out the elution series. Detection was achieved via PDA and MS.

\section{Click Pull-down Methods}

\section{Click Pulldown in BCG}

BCG stocks were grown in liquid agar to an OD of 0.5. Bacterial stocks were transferred in to culture flasks, administered click probes at 10x MIC and incubated at $37^{\circ} \mathrm{C}$ for 48 hours, then harvested via centrifugation. The pellets were twice washed in PBS and centrifuged. The pellets were then resuspended in $700 \mu \mathrm{L}$ of PBS for beadbeating. The resultant solution was clarified via centrifugation and the supernatant decanted. The supernatant was separated in two fractions via size exclusion membrane $(2,000 \mathrm{amu})$. All fractions were collected and stored frozen until the completion of the experiment. 
All samples were thawed at room temperature and briefly vortexed to homogenize. The extracts were then transferred into a microwave tube containing of 1:1 $\mathrm{CH}_{3} \mathrm{OH}: \mathrm{H}_{2} \mathrm{O}(2.5 \mathrm{~mL}) 100 \mathrm{uL}$ of previously prepared $\left(100 \mu \mathrm{g} / \mathrm{mL}\right.$ in $\left.\mathrm{CH}_{3} \mathrm{OH}\right)$ tag was add to the tube with a catalytic quantity of TBTA. The samples were allowed to react stirring slowly in a $37^{\circ} \mathrm{C}$ oil bath overnight. Upon completion the organic solvents were removed from the reaction mixture via rotovap under soft vacuum the aqueous solutions were filtered and vialed for UPLC or SDS/PAGE Analysis.

\section{HPLC Separation of Click Conjugation Products}

All samples were dissolved in $\mathrm{GC} / \mathrm{MS}$ grade $\mathrm{CH}_{3} \mathrm{OH}$ and transferred to autosampler vials. Separations were achieved on a Grace Davidson Prevail C18 Select Rocket Column $(53 \mathrm{~mm} \times 7 \mathrm{~mm} \times 3 \mu \mathrm{m})$ at $50^{\circ} \mathrm{C}$, flowing $2.5 \mathrm{~mL} / \mathrm{min}$ of $\mathrm{H}_{2} \mathrm{O}+0.1 \%$ Formic acid (A) and ACN+ 0.1\% Formic Acid (B). The first 12.5 minutes were maintained at $10 \%$ organic followed by a linear ramp to $95 \%$ organic over 45 minutes then held at high organic for an additional 12.5 minutes resulting in a 1 hour total chromatographic elution. All fractions were collected in glass tubes then turbovaped to dryness. The samples flagged through UV and ELSD were reanalyzed via UPLC/MS. Fractions found to contain lipid moieties were divided 1:10 for bioanalysis and archival respectively.

\section{MS Identification of Heavy Fractions}

The protein gel bands were reduced and alkylated with iodoacetamide and digested with Trypsin. Mass spectrometric analysis was performed using an LTQ Orbitrap Mass Spectrometer from Thermo Electron (San Jose, CA). This instrument employs electrospray ionization (ESI), in conjunction with an Orbitrap mass analyzer. The digest was introduced into the instrument via on line chromatography using reverse phase (C18) ultra-high pressure liquid chromatography on the nanoAcquity (Waters, MA). The column used was a Waters BEHC18 with an I.D. of 75 um and bed length of $10 \mathrm{~cm}$. The particle size was $1.7 \mathrm{um}$. Peptides were gradient eluted into the linear ion trap through a non-coated spray needle with voltage applied to the liquid by increasing the concentration of acetonitrile. Data acquisition involved acquiring the peptide mass (MS) spectra followed by fragmentation of the peptide to produce MS/MS spectra that provides information about the peptide sequence. Database searches were performed using LTQ.raw files in combination with the Mascot search engine. Protein/peptide assignments were made on the basis of MS/MS spectra. 


\section{LIST OF REFERENCES}

1. McLafferty, F. W., Trends in analytical instrumentation. Science 1984, 226

(Copyright (C) 2012 U.S. National Library of Medicine.), 251-3.

2. Griffiths, J., A brief history of mass spectrometry. Anal. Chem. (Washington, DC, U. S.) 2008, 80 (Copyright (C) 2012 American Chemical Society (ACS). All Rights Reserved.), 5678-5683.

3. Thomson, J. J., Electronic waves. Philos. Mag. (1798-1977) 1939, 27 (Copyright (C) 2012 American Chemical Society (ACS). All Rights Reserved.), 1-32.

4. Goldstein, E., On the changes of color of certain salts under the action of cathode rays. Sitzungsber. Akad. Wiss. Berlin 1895 (Copyright (C) 2012 American Chemical Society (ACS). All Rights Reserved.), 467.

5. McLennan, J. C., Electrical conductivity in gases through which cathode rays pass. Z. Phys. Chem., Stoechiom. Verwandtschaftsl. 1901, 37 (Copyright (C) 2012 American Chemical Society (ACS). All Rights Reserved.), 513.

6. Thomson, J. J., The discharge of electricity through gases. Charles Scribner's Sons: 1898 ; p x pp. and 203 pp.

7. Thomson, J. J., On the masses of the ions in gases at low pressures. Philos. Mag. (1798-1977) 1899, 48 (Copyright (C) 2012 American Chemical Society (ACS). All Rights Reserved.), 547.

8. Thomson, J. J., The Velocity of Secondary Cathode Rays from Gases. Proc. Cambridge Philos. Soc. 1909, 14 (Copyright (C) 2012 American Chemical Society (ACS). All Rights Reserved.), 541-5.

9. Thomson, J. J., Scattering of Rapidly Moving Electrified Particles. Proc. Cambridge Philos. Soc. 1910, 15 (Copyright (C) 2012 American Chemical Society (ACS). All Rights Reserved.), 465-71.

10. Thomson, J. J., The structure of the atom. Philos. Mag. (1798-1977) 1914, 26 (Copyright (C) 2012 American Chemical Society (ACS). All Rights Reserved.), 792-9.

11. Thomson, J. J., The Appearance of Helium and Neon in Vacuum Tubes. Nature (London, U. K.) 1913, 90 (Copyright (C) 2012 American Chemical Society (ACS). All Rights Reserved.), 645-7. 
12. Aston, F. W., The constitution of the elements. Nature (London, U. K.) 1919, 104 (Copyright (C) 2012 American Chemical Society (ACS). All Rights Reserved.), 393.

13. Linder, E. G., Mass-spectrograph study of the ionization and dissociation by electron impact of benzene and carbon disulfide. Phys. Rev. 1932, 41 (Copyright (C) 2012 American Chemical Society (ACS). All Rights Reserved.), 149-53.

14. McLafferty, F. W., Mass spectrometric analysis. Broad applicability to chemical research. Anal. Chem. 1956, 28 (Copyright (C) 2012 American Chemical Society (ACS). All Rights Reserved.), 306-16.

15. March, R. E.; Todd, J. F. J. In An appreciation and historical survey of mass spectrometry, CRC Press: 2010; pp 3-168.

16. Washburn, H. W.; Wiley, H. F.; Rock, S. M., The mass spectrometer as an analytical tool. Ind. Eng. Chem., Anal. Ed. 1943, 15 (Copyright (C) 2012 American Chemical Society (ACS). All Rights Reserved.), 541-7.

17. Pelchowitch, I., A high-resolution mass spectrometer with variable-bandwidth measuring circuits. Philips Res. Rep. 1954, 9 (Copyright (C) 2012 American Chemical Society (ACS). All Rights Reserved.), 1-41.

18. Rosenblum, E. S., A sector-type double-focusing magnetic spectrometer. Phys. Rev. 1949, 75 (Copyright (C) 2012 American Chemical Society (ACS). All Rights Reserved.), 1628.

19. Hipple, J. A., Peak contour and half-life of metastable ions appearing in mass spectra. Phys. Rev. 1947, 71 (Copyright (C) 2012 American Chemical Society (ACS). All Rights Reserved.), 594-9.

20. Baldock, R.; Sites, J. R., Mass-spectrometer studies of the dissociation of some metal carbonyls. U. S. A. E. C. 1951, Y-761 (Copyright (C) 2012 American Chemical Society (ACS). All Rights Reserved.), 12 pp.

21. Marsden, D. G. H., Panoramic mass spectrometer. Rev. Sci. Instrum. 1962, 33 (Copyright (C) 2012 American Chemical Society (ACS). All Rights Reserved.), 288-93.

22. Vick, F. A., Recent advances in science: physics. Sci. Prog. (St. Albans, U. K.) 1948, 36 (Copyright (C) 2012 American Chemical Society (ACS). All Rights Reserved.), 78-85.

23. Inghram, M. G., Modern mass spectroscopy. Advances in Electronics (Academic Press Inc., New York) 1948, 1 (Copyright (C) 2012 American Chemical Society (ACS). All Rights Reserved.), 219-68. 
24. Burmaster, K. E.; Evans, E. C., The mass spectrometer. Its accomplishments, applications, and future. Instruments 1950, 23 (Copyright (C) 2012 American Chemical Society (ACS). All Rights Reserved.), 242-5.

25. Chulanovskii, V. M., Molecular analysis by means of mass spectra. Zavod. Lab. 1950, 16 (Copyright (C) 2012 American Chemical Society (ACS). All Rights Reserved.), 566-77.

26. Hagstrum, H. D., Ionization by electron impact. Natl. Bur. Stand. Circ. (U. S.) 1953, Circ. No. 522 (Copyright (C) 2012 American Chemical Society (ACS). All Rights Reserved.), 193-9.

27. Scribner, B. F., Emission spectroscopy. Anal. Chem. 1958, 30 (Copyright (C) 2012 American Chemical Society (ACS). All Rights Reserved.), 596-604.

28. Stamm, R. F., Raman spectroscopy. Anal. Chem. 1954, 26 (Copyright (C) 2012 American Chemical Society (ACS). All Rights Reserved.), 49-53.

; (b) DeFord, D. D.; Bowers, R. C., Electroanalysis and coulometric analysis. Anal. Chem. 1958, 30 (Copyright (C) 2012 American Chemical Society (ACS). All Rights Reserved.), 613-19.

29. Pavlenko, V. A., Apparatus for automatic gas analysis and mass spectrometers. Trudy, Nauch.-Tekh. Obshchestvo Priborstroitel. Prom. 1959, (Copyright (C) 2012 American Chemical Society (ACS). All Rights Reserved.), 96-124.

30. LeTourneau, R. L., Analysis of petroleum. Prepr. - Am. Chem. Soc., Div. Pet. Chem. 1961, 6 (Copyright (C) 2012 American Chemical Society (ACS). All Rights Reserved.), 15-25.

31. Testerman, M. K., Mass spectrometry: new instruments and techniques. Mass Spectrom., New Instrum. Tech. 1960, 7 (Copyright (C) 2012 American Chemical Society (ACS). All Rights Reserved.), 51 pp.

32. Merten, F., Measuring equipment for the laboratory. Chem. Ing. Tech. 1961, 33 (Copyright (C) 2012 American Chemical Society (ACS). All Rights Reserved.), 271-80.

33. Berry, C. E., High resolution in conventional $180^{\circ}$ mass spectrometers. Natl. Bur. Stand. Circ. (U. S.) 1953, No. 522 (Copyright (C) 2012 American Chemical Society (ACS). All Rights Reserved.), 99-105.

34. Dibeler, V. H., Mass spectrometry. Anal. Chem. 1954, 26 (Copyright (C) 2012 American Chemical Society (ACS). All Rights Reserved.), 58-65. 
35. Hinterberger, H., Newer developments in mass spectroscopy. Experientia 1956, 12 (Copyright (C) 2012 American Chemical Society (ACS). All Rights Reserved.), 449-71.

36. Sharkey, A. G., Jr., Mass spectrometry: new instruments and techniques. Mass Spectrom., New Instrum. Tech. 1962, 10 (Copyright (C) 2012 American Chemical Society (ACS). All Rights Reserved.), 65 pp.

37. Robertson, A. J. B., Physics in the service of chemistry. Mass spectrometry. $J$. Roy. Inst. Chem. 1958, 82 (Copyright (C) 2012 American Chemical Society (ACS). All Rights Reserved.), 295-308.

38. Howlett, K. E., Recent advances in science: Physical chemistry. Sci. Progr. (London) 1963, 51 (Copyright (C) 2012 American Chemical Society (ACS). All Rights Reserved.), 429-50.

39. Goudsmit, S. A., Time-of-flight mass spectrometer. Phys. Rev. 1948, 74 (Copyright (C) 2012 American Chemical Society (ACS). All Rights Reserved.), 622-3.

40. Langmuir, R. V. Mass spectrometry. US2780728, 1957.

41. Widmer, H.; Gaumann, T., The combination of a gas chromatograph with a mass spectrograph. Helv. Chim. Acta 1962, 45 (Copyright (C) 2012 American Chemical Society (ACS). All Rights Reserved.), 2175-85.

42. Studier, M. H., Continuous ion source for a time-of-flight mass spectrometer. Rev. Sci. Instrum. 1963, 34 (Copyright (C) 2012 American Chemical Society (ACS). All Rights Reserved.), 1367-70.

43. Wragg, J. S.; Johnson, G. W., Pharmaceutical Analysis. Applications of highpressure liquid chromatography. Pharm. J. 1974, 213 (Copyright (C) 2012 American Chemical Society (ACS). All Rights Reserved.), 601-4, 613.

44. Gallagher, R. T.; Chapman, J. R.; Mann, M., Design and performance of an electrospray ionization source for a doubly-focusing magnetic sector mass spectrometer. Rapid Commun. Mass Spectrom. 1990, 4 (Copyright (C) 2012 American Chemical Society (ACS). All Rights Reserved.), 369-72.

45. March, R. E., Quadrupole ion trap mass spectrometry: theory, simulation, recent developments and applications. Rapid Commun. Mass Spectrom. 1998, 12 (Copyright (C) 2012 American Chemical Society (ACS). All Rights Reserved.), 1543-1554. 
46. Fenn, J. B.; Mann, M.; Meng, C. K.; Wong, S. F.; Whitehouse, C. M., Electrospray ionization for mass spectrometry of large biomolecules. Science 1989, 246 (Copyright (C) 2012 U.S. National Library of Medicine.), 64-71.

47. Fenn, J. B.; Rosell, J.; Nohmi, T.; Shen, S.; Banks, F. J., Jr., Electrospray ion formation: desorption versus desertion. ACS Symp. Ser. 1996, 619 (Copyright (C) 2012 American Chemical Society (ACS). All Rights Reserved.), 60-80.

48. Meng, C. K.; Nohmi, T.; Fuerstenau, S. D.; Fenn, J. B.; Shen, S.; Banks, J. F., Jr. Electrospray ionization. A new window on the solution scene; 0195-721X; Yale Univ.: 1992; pp 339-42.

49. Labowsky, M.; Fenn, J. B.; Fernandez, d. 1. M. J., A continuum model for ion evaporation from a drop: effect of curvature and charge on ion solvation energy. Anal. Chim. Acta 2000, 406 (Copyright (C) 2012 American Chemical Society (ACS). All Rights Reserved.), 105-118.

50. Meng, C. K.; Fenn, J. B., Analyzing organic molecules with electrospray mass spectrometry. Am Biotechnol Lab 1990, 8 (Copyright (C) 2012 U.S. National Library of Medicine.), 54-60.

51. Meng, C. K.; Fenn, J. B., Analyzing organic molecules with electrospray mass spectrometry. Am. Biotechnol. Lab. 1990, 8 (Copyright (C) 2012 American Chemical Society (ACS). All Rights Reserved.), 54-60.

52. Louris, J. N.; Amy, J. W.; Ridley, T. Y.; Cooks, R. G., Injection of ions into a quadrupole ion trap mass spectrometer. Int. J. Mass Spectrom. Ion Processes 1989, 88 (Copyright (C) 2012 American Chemical Society (ACS). All Rights Reserved.), 97-111.

53. Cooks, R. G.; Hoke, S. H., II; Morand, K. L.; Lammert, S. A., Mass spectrometers: instrumentation. Int. J. Mass Spectrom. Ion Processes 1992, 118 119 (Copyright (C) 2012 American Chemical Society (ACS). All Rights Reserved.), 1-36.

54. Woodward, C. E.; Crawford, C. K., Design of a quadrupole mass spectrometer. NASA (Natl. Aeron. Space Admin.), Doc. 1963, N63-13460 (Copyright (C) 2012 American Chemical Society (ACS). All Rights Reserved.), 32 pp.

55. Slobodenyuk, G. I., Solution of some equations characterizing the main parameters of a mass spectrometer. Zh. Tekh. Fiz. 1967, 37 (Copyright (C) 2012 American Chemical Society (ACS). All Rights Reserved.), 964-70. 
56. Brunee, C.; Delgmann, L.; Kronenberger, K., A quadrupole high-frequency mass spectrometer for applications in vacuum technology. Vak.-Tech. 1964, 13 (Copyright (C) 2012 American Chemical Society (ACS). All Rights Reserved.), $35-42$.

57. Brunnee, C.; Delgmann, L.; Kronenberger, K., The Atlas quadrupole mass spectrometer. Publ. Group. Avan. Methodes Spectrog. 1964, (Copyright (C) 2012 American Chemical Society (ACS). All Rights Reserved.), 177-88.

58. Gray, C. G.; Karl, G.; Novikov, V. A., Quadrupolar contact fields: Theory and applications. Am. J. Phys. 2009, 77 (Copyright (C) 2012 American Chemical Society (ACS). All Rights Reserved.), 807-817.

59. Jones, E. B. In Quadrupole, triple-quadrupole, and hybrid linear ion trap mass spectrometers for metabolite analysis, John Wiley \& Sons, Inc.: 2009; pp 123157.

60. Miller, P. E.; Denton, M. B., The quadrupole mass filter: basic operating concepts. J. Chem. Educ. 1986, 63 (Copyright (C) 2012 American Chemical Society (ACS). All Rights Reserved.), 617-22.

61. March, R. E.; Londry, F. A. In Theory of quadrupole mass spectrometry, CRC: 1995; pp 25-48.

62. Stafford, G. C., Jr.; Kelley, P. E.; Syka, J. E. P.; Reynolds, W. E.; Todd, J. F. J., Recent improvements in and analytical applications of advanced ion trap technology. Int. J. Mass Spectrom. Ion Processes 1984, 60 (Copyright (C) 2012 American Chemical Society (ACS). All Rights Reserved.), 85-98.

63. Chen, L.; Wang, T. C.; Ricca, T. L.; Marshall, A. G., Phase-modulated stored waveform inverse Fourier transform excitation for trapped ion mass spectrometry. Anal Chem 1987, 59 (Copyright (C) 2012 U.S. National Library of Medicine.), 449-54.

64. Chen, L.; Wang, T. C. L.; Ricca, T. L.; Marshall, A. G., Phase-modulated stored waveform inverse Fourier transform excitation for trapped ion mass spectrometry. Anal. Chem. 1987, 59 (Copyright (C) 2012 American Chemical Society (ACS). All Rights Reserved.), 449-54.

65. Cooks, R. G.; Kaiser, R. E., Jr., Quadrupole ion trap mass spectrometry. Acc. Chem. Res. 1990, 23 (Copyright (C) 2012 American Chemical Society (ACS). All Rights Reserved.), 213-19. 
66. Kaiser, R. E., Jr.; Cooks, R. G.; Stafford, G. C., Jr.; Syka, J. E. P.; Hemberger, P. H., Operation of a quadrupole ion trap mass spectrometer to achieve high mass/charge ratios. Int. J. Mass Spectrom. Ion Processes 1991, 106 (Copyright (C) 2012 American Chemical Society (ACS). All Rights Reserved.), 79-115.

67. Todd, J. F. J., Ion trap mass spectrometer - past, present, and future(?). Mass Spectrom. Rev. 1991, 10 (Copyright (C) 2012 American Chemical Society (ACS). All Rights Reserved.), 3-52.

68. Doroshenko, V. M.; Cotter, R. J., A new method of trapping ions produced by matrix-assisted laser desorption ionization in a quadrupole ion trap. Rapid Commun. Mass Spectrom. 1993, 7 (Copyright (C) 2012 American Chemical Society (ACS). All Rights Reserved.), 822-7.

69. Desmet, G., The history of chromatography: John Knox, a pioneer of both gas and liquid chromatography. LCGC North Am. 2011, 29 (Copyright (C) 2012 American Chemical Society (ACS). All Rights Reserved.), 996,998,1000,1002,1004.

70. Di, F. F., Brief history of chromatography. Lab. 2000 2004, 18 (Copyright (C) 2012 American Chemical Society (ACS). All Rights Reserved.), 62-63.

71. Kouros, G., History of chromatography: from Tswett to chromatographic analysis. Chem. Chron., Genike Ekdose 1998, 60 (Copyright (C) 2012 American Chemical Society (ACS). All Rights Reserved.), 110-113.

72. Voegelin, W.; Vetterli, W. A.; Napier, S., History of chromatography. Ciba Rev. 1966, (Copyright (C) 2012 American Chemical Society (ACS). All Rights Reserved.), 2-10.

73. Grob, K., The history of chromatography. Kurt Grob - One of the fathers of capillary GC. LCGC North Am. 2008, 26 (Copyright (C) 2012 American Chemical Society (ACS). All Rights Reserved.), 1022,1024,1026-1030.

74. Homing, E. C.; Heuvel, W. J. A. V., Gas chromatography. Annu. Rev. Biochem. 1963, 32 (Copyright (C) 2012 American Chemical Society (ACS). All Rights Reserved.), 709-54.

75. Ridgeon, P. J., An introduction to gas chromatography. Birmingham Univ. Chem. Eng. 1965, 16 (Copyright (C) 2012 American Chemical Society (ACS). All Rights Reserved.), 95-8.

76. Luong, J.; Gras, R.; Mustacich, R.; Cortes, H., Low thermal mass gas chromatography: principles and applications. J Chromatogr Sci 2006, 44 (Copyright (C) 2012 U.S. National Library of Medicine.), 253-61. 
77. Cao, X. L.; Casey, V., Improved method for the determination of benzene in soft drinks at sub-ppb levels. Food Addit Contam Part A Chem Anal Control Expo Risk Assess 2008, 25 (Copyright (C) 2012 U.S. National Library of Medicine.), 401-5.

78. Song, S. M.; Marriott, P. J., Analytical aspects of modern gas chromatography: mass spectrometry. Chromatogr. Sci. Ser. 2012, 104 (Copyright (C) 2012 American Chemical Society (ACS). All Rights Reserved.), 31-59.

79. Thompson, A. E., Flame ionization detector for gas chromatography. $J$. Chromatogr. 1959, 2 (Copyright (C) 2012 American Chemical Society (ACS). All Rights Reserved.), 148-54.

80. Andreatch, A. J.; Feinland, R., Continuous trace hydrocarbon analysis by flame ionization. Anal. Chem. 1960, 32 (Copyright (C) 2012 American Chemical Society (ACS). All Rights Reserved.), 1021-4.

81. Jeong, I.-S.; Kwak, B.-M.; Ahn, J.-H.; Leem, D.; Yoon, T.; Yoon, C.; Jeong, J.; Park, J.-M.; Kim, J.-M., A Novel Sample Preparation Method Using Rapid Nonheated Saponification Method for the Determination of Cholesterol in Emulsified Foods. J Food Sci 2012, (Copyright (C) 2012 U.S. National Library of Medicine.).

82. Lovelock, J. E.; Gregory, N. L., Electron capture ionization detectors. Gas Chromatog., Intern. Symp., 1961 1962, 3 (Copyright (C) 2012 American Chemical Society (ACS). All Rights Reserved.), 219-29.

83. Robinson, J.; Richardson, A., Separation of chlorinated insecticides and related compounds. Chem. Ind. (London, U. K.) 1963, (Copyright (C) 2012 American Chemical Society (ACS). All Rights Reserved.), 1460-2.

84. Gregory, N. L., Gas dilution system for control of detector response in gas chromatography. J. Chromatogr. 1964, 13 (Copyright (C) 2012 American Chemical Society (ACS). All Rights Reserved.), 33-9.

85. An, E.-m.; Shin, H.-S., Gas chromatographic determination of pesticide residues using electron-capture detector and mass spectrometry. Food Sci. Biotechnol. 2011, 20 (Copyright (C) 2012 American Chemical Society (ACS). All Rights Reserved.), 1299-1306.

86. Brown, P. R., Use of high-pressure liquid chromatography in pharmacology and toxicology. Adv. Chromatogr. 1975, 12 (Copyright (C) 2012 American Chemical Society (ACS). All Rights Reserved.), 1-30. 
87. Javier, V. F., Advantages of high-pressure liquid chromatography. IQ 1974, 64 (Copyright (C) 2012 American Chemical Society (ACS). All Rights Reserved.), 31-4.

88. Boeler, J. B., High-pressure liquid chromatography - an analytical technique with broad applications in research and industry. Kjemi 1974, 34 (Copyright (C) 2012 American Chemical Society (ACS). All Rights Reserved.), 8-10.

89. Belue, G. P.; McGinnis, G. D., High pressure liquid chromatography of carbohydrates. J. Chromatogr. 1974, 97 (Copyright (C) 2012 American Chemical Society (ACS). All Rights Reserved.), 25-31.

90. Leppard, J. P.; Reid, E., Physical methods and techniques. IV. Chromatography. A. High-pressure liquid chromatography. Annu. Rep. Prog. Chem., Sect. B 1975, 71 (Copyright (C) 2012 American Chemical Society (ACS). All Rights Reserved.), 44-55.

91. Beyer, W. F.; Gleason, D. D., Laboratory automation of high-pressure liquid chromatography. J. Pharm. Sci. 1975, 64 (Copyright (C) 2012 American Chemical Society (ACS). All Rights Reserved.), 1557-60.

92. Berry, L.; Karger, B. L., Pumps and injectors for modern liquid chromatography. Anal. Chem. 1973, 45 (Copyright (C) 2012 American Chemical Society (ACS). All Rights Reserved.), 819A-826A, 828A.

93. Hunt, R. J.; Wadsworth, J. H. F.; Hubbard, F. V. Gas chromatography oven. GB2141358A, 1984.

94. Knox, J. H., Equipment for high-pressure liquid chromatography. Biochem J 1972, 130 (Copyright (C) 2012 U.S. National Library of Medicine.), 56P-57P.

95. Mollica, J. A.; Strusz, R. F., Analysis of corticosteroid creams and ointments by high pressure liquid chromatography. J Pharm Sci 1972, 61 (Copyright (C) 2012 U.S. National Library of Medicine.), 444-7.

96. Rzeszotarski, W. J.; Mauger, A. B., Reversed-phase high-pressure liquid chromatography of actinomycins. J Chromatogr 1973, 86 (Copyright (C) 2012 U.S. National Library of Medicine.), 246-9.

97. Cox, G. B., High pressure liquid chromatography. An introduction and applications review. Misc. Rep. - Lab. Gov. Chem. (G. B.) 1973, 1 (Copyright (C) 2012 American Chemical Society (ACS). All Rights Reserved.), 67 pp.

98. Schomburg, G., Detection in high pressure liquid chromatography. Fresenius' $Z$. Anal. Chem. 1975, 277 (Copyright (C) 2012 American Chemical Society (ACS). All Rights Reserved.), 275-87. 
99. Richtr, V., Use of ultraviolet and infrared spectroscopy in organic chemistry. $S b$. Pedagog. Fak. Plzni, [Ser.]: Chem. 1976, 10 (Copyright (C) 2012 American Chemical Society (ACS). All Rights Reserved.), 35-57.

100. Lafosse, M.; Dreux, M.; Morin-Allory, L., Application fields of a new evaporative light scattering detector for high-performance liquid chromatography and supercritical fluid chromatography. J. Chromatogr. 1987, 404 (Copyright (C) 2012 American Chemical Society (ACS). All Rights Reserved.), 95-105.

101. Mourey, T. H.; Oppenheimer, L. E., Principles of operation of an evaporative light-scattering detector for liquid chromatography. Anal. Chem. 1984, 56 (Copyright (C) 2012 American Chemical Society (ACS). All Rights Reserved.), 2427-34.

102. Bear, G. R., Universal detection and quantitation of surfactants by highperformance liquid chromatography by means of the evaporative light-scattering detector. J. Chromatogr. 1988, 459 (Copyright (C) 2012 American Chemical Society (ACS). All Rights Reserved.), 91-107.

103. Wood, E. J. F., Classification of bacteria. Nature 1949, 164 (Copyright (C) 2012 U.S. National Library of Medicine.), 867-9.

104. Demain, A. L., Induction of microbial secondary metabolism. Int. Microbiol. 1998, 1 (Copyright (C) 2012 American Chemical Society (ACS). All Rights Reserved.), 259-264.

105. Kanistanon, D.; Hajjar, A. M.; Pelletier, M. R.; Gallagher, L. A.; Kalhorn, T.; Shaffer, S. A.; Goodlett, D. R.; Rohmer, L.; Brittnacher, M. J.; Skerrett, S. J.; Ernst, R. K., A Francisella mutant in lipid A carbohydrate modification elicits protective immunity. PLoS Pathog. 2008, 4 (Copyright (C) 2012 American Chemical Society (ACS). All Rights Reserved.), No pp. given.

106. Folch-Pi, J.; Lees, M.; Stanley, G. H. S., A simple method for the isolation and purification of total lipides from animal tissues. J. Biol. Chem. 1957, 226 (Copyright (C) 2012 American Chemical Society (ACS). All Rights Reserved.), 497-509.

107. Li, Y.; Powell, D. A.; Shaffer, S. A.; Rasko, D. A.; Pelletier, M. R.; Leszyk, J. D.; Scott, A. J.; Masoudi, A.; Goodlett, D. R.; Wang, X.; Raetz, C. R. H.; Ernst, R. K., LPS remodeling is an evolved survival strategy for bacteria. Proc. Natl. Acad. Sci. U. S. A. 2012, 109 (Copyright (C) 2012 American Chemical Society (ACS). All Rights Reserved.), 13877.

108. Frank, M. a. S., Jerrod 2D TLC, Direct Injection LTQ-MS of Ft Lipid Extracts. Scarborough, J., Ed. St Jude Children's Research Hospital University of Tennessee Health Science Center, 2012. 
109. Asiedu, K.; Etuaful, S., Socioeconomic implications of Buruli ulcer in Ghana: a three-year review. Am J Trop Med Hyg 1998, 59 (Copyright (C) 2012 U.S. National Library of Medicine.), 1015-22.

110. George, K. M.; Pascopella, L.; Welty, D. M.; Small, P. L. C., A Mycobacterium ulcerans toxin, mycolactone, causes apoptosis in guinea pig ulcers and tissue culture cells. Infect. Immun. 2000, 68 (Copyright (C) 2012 American Chemical Society (ACS). All Rights Reserved.), 877-883.

111. Mve-Obiang, A.; Lee, R. E.; Umstot, E. S.; Trott, K. A.; Grammer, T. C.; Parker, J. M.; Ranger, B. S.; Grainger, R.; Mahrous, E. A.; Small, P. L. C., A newly discovered mycobacterial pathogen isolated from laboratory colonies of Xenopus species with lethal infections produces a novel form of mycolactone, the Mycobacterium ulcerans macrolide toxin. Infect. Immun. 2005, 73 (Copyright (C) 2012 American Chemical Society (ACS). All Rights Reserved.), 3307-3312.

112. Marion, E.; Prado, S.; Cano, C.; Babonneau, J.; Ghamrawi, S.; Marsollier, L., Photodegradation of the Mycobacterium ulcerans toxin, mycolactones: considerations for handling and storage. PLoS One 2012, 7 (Copyright (C) 2012 American Chemical Society (ACS). All Rights Reserved.), e33600.

113. Groenberg, A.; Zettergren, L.; Bergh, K.; Staahle, M.; Heilborn, J.; Aengeby, K.; Small, P. L.; Akuffo, H.; Britton, S., Antioxidants protect keratinocytes against M. ulcerans mycolactone cytotoxicity. PLoS One 2010, 5 (Copyright (C) 2012 American Chemical Society (ACS). All Rights Reserved.), No pp. given.

114. Yotsu, R. R.; Nakanaga, K.; Hoshino, Y.; Suzuki, K.; Ishii, N., Buruli ulcer and current situation in Japan: a new emerging cutaneous Mycobacterium infection. $J$. Dermatol. 2012, 39 (Copyright (C) 2012 American Chemical Society (ACS). All Rights Reserved.), 587-593.

115. Jorgensen, J. H.; Ferraro, M. J., Antimicrobial susceptibility testing: a review of general principles and contemporary practices. Clin. Infect. Dis. 2009, 49 (Copyright (C) 2012 American Chemical Society (ACS). All Rights Reserved.), 1749-1755.

116. Anon, Comprehensive Natural Products II: Chemistry and Biology, Volumes 1-10 Edited by Lewis Mander and Hung-Wen Liu. J. Am. Chem. Soc. 2010, 132 (Copyright (C) 2012 American Chemical Society (ACS). All Rights Reserved.), 9929.

117. Greenwood, D., Tarnished gold: sixty years of antimicrobial drug use and misuse. J Med Microbiol 1995, 43 (Copyright (C) 2012 U.S. National Library of Medicine.), 395-6. 
118. Bonhoeffer, S.; Lipsitch, M.; Levin, B. R., Evaluating treatment protocols to prevent antibiotic resistance. Proc. Natl. Acad. Sci. U. S. A. 1997, 94 (Copyright (C) 2012 American Chemical Society (ACS). All Rights Reserved.), 1210612111.

119. Klem, C.; Dasta, J. F., Efforts of pharmacy to reduce antibiotic resistance. New Horiz 1996, 4 (Copyright (C) 2012 U.S. National Library of Medicine.), 377-84.

120. Jarvis, W. R., Preventing the emergence of multidrug-resistant microorganisms through antimicrobial use controls: the complexity of the problem. Infect Control Hosp Epidemiol 1996, 17 (Copyright (C) 2012 U.S. National Library of Medicine.), 490-5.

121. Matsuoka, M.; Aye, K. S.; Kyaw, K.; Tan, E. V.; Balagon, M. V.; Saunderson, P.; Gelber, R.; Makino, M.; Nakajima, C.; Suzuki, Y., A novel method for simple detection of mutations conferring drug resistance in Mycobacterium leprae, based on a DNA microarray, and its applicability in developing countries. J. Med. Microbiol. 2008, 57 (Copyright (C) 2012 American Chemical Society (ACS). All Rights Reserved.), 1213-1219.

122. Schwalbe, R. S.; Stapleton, J. T.; Gilligan, P. H., Emergence of vancomycin resistance in coagulase-negative staphylococci. $N$ Engl J Med 1987, 316 (Copyright (C) 2012 U.S. National Library of Medicine.), 927-31.

123. Billstein, S. A., How the pharmaceutical industry brings an antibiotic drug to market in the United States. Antimicrob. Agents Chemother. 1994, 38 (Copyright (C) 2012 American Chemical Society (ACS). All Rights Reserved.), 2679-82.

124. Morris, J. G., Jr.; Shay, D. K.; Hebden, J. N.; McCarter, R. J., Jr.; Perdue, B. E.; Jarvis, W.; Johnson, J. A.; Dowling, T. C.; Polish, L. B.; Schwalbe, R. S., Enterococci resistant to multiple antimicrobial agents, including vancomycin: establishment of endemicity in a university medical center. Ann. Intern. Med. 1995, 123 (Copyright (C) 2012 American Chemical Society (ACS). All Rights Reserved.), 250-9.

125. Nelson, R., Antibiotic development pipeline runs dry. New drugs to fight resistant organisms are not being developed, experts say. Lancet 2003, 362 (Copyright (C) 2012 U.S. National Library of Medicine.), 1726-7.

126. Mitka, M., Antibiotic pipeline. JAMA, J. Am. Med. Assoc. 2012, 307 (Copyright (C) 2012 American Chemical Society (ACS). All Rights Reserved.), 1476.

127. Jabes, D., The antibiotic R\&D pipeline: an update. Curr Opin Microbiol 2011, 14 (Copyright (C) 2012 U.S. National Library of Medicine.), 564-9. 
128. Mitchison, D. A., Prevention of drug resistance by combined drug treatment of tuberculosis. Handb Exp Pharmacol 2012, (Copyright (C) 2012 U.S. National Library of Medicine.), 87-98.

129. Sahbazian, B.; Weis, S. E., Treatment of active tuberculosis: challenges and prospects. Clin Chest Med 2005, 26 (Copyright (C) 2012 U.S. National Library of Medicine.), 273-82, vi.

130. Schaberg, T.; Forssbohm, M.; Hauer, B.; Kirsten, D.; Kropp, R.; Loddenkemper, R.; Magdorf, K.; Rieder, H.; Sagebiel, D.; Urbanczik, R., Guidelines for drug treatment of tuberculosis in adults and childhood. Pneumologie 2001, 55 (Copyright (C) 2012 U.S. National Library of Medicine.), 494-511.

131. Keshavjee, S.; Farmer, P. E., Tuberculosis, drug resistance, and the history of modern medicine. N. Engl. J. Med. 2012, 367 (Copyright (C) 2012 American Chemical Society (ACS). All Rights Reserved.), 931-936.

132. Anonymous, $\mathrm{CDC}$ recommends four-drug treatment of tuberculosis in multidrugresistant era. Am Fam Physician 1993, 48 (Copyright (C) 2012 U.S. National Library of Medicine.), 671-2.

133. Davidson, P. T.; Le, H. Q., Drug treatment of tuberculosis--1992. Drugs 1992, 43 (Copyright (C) 2012 U.S. National Library of Medicine.), 651-73.

134. Zhang, Y., Advances in the treatment of tuberculosis. Clin. Pharmacol. Ther. (N. Y., NY, U. S.) 2007, 82 (Copyright (C) 2012 American Chemical Society (ACS). All Rights Reserved.), 595-600.

135. Holland, D. P.; Sanders, G. D.; Hamilton, C. D.; Stout, J. E., Strategies for treating latent multiple-drug resistant tuberculosis: a decision analysis. PLoS One 2012, 7 (Copyright (C) 2012 American Chemical Society (ACS). All Rights Reserved.), e30194.

136. Gumbo, T., New susceptibility breakpoints for first-line antituberculosis drugs based on antimicrobial pharmacokinetic/pharmacodynamic science and population pharmacokinetic variability. Antimicrob Agents Chemother 2010, 54 (Copyright (C) 2012 U.S. National Library of Medicine.), 1484-91.

137. Gillespie, S. H.; Singh, K., XDR-TB, what is it; how is it treated; and why is therapeutic failure so high? Recent Pat. Anti-Infect. Drug Discovery 2011, 6 (Copyright (C) 2012 American Chemical Society (ACS). All Rights Reserved.), 77-83.

138. Young, D. B., Chaperonins and the immune response. Semin Cell Biol 1990, 1 (Copyright (C) 2012 U.S. National Library of Medicine.), 27-35. 
139. Kaufmann, S. H. E., Immunity to bacteria. Curr. Opin. Immunol. 1991, 2 (Copyright (C) 2012 American Chemical Society (ACS). All Rights Reserved.), 353-9.

140. Erb, K. J.; Holloway, J. W.; Le, G. G., Innate immunity: mast cells in the front line. Curr. Biol. 1996, 6 (Copyright (C) 2012 American Chemical Society (ACS). All Rights Reserved.), 941-942.

141. Shin, J. S.; Gao, Z.; Abraham, S. N., Bacteria-host cell interaction mediated by cellular cholesterol/glycolipid-enriched microdomains. Biosci Rep 1999, 19 (Copyright (C) 2012 U.S. National Library of Medicine.), 421-32.

142. Harding, C. V.; Ramachandra, L.; Wick, M. J., Interaction of bacteria with antigen presenting cells: influences on antigen presentation and antibacterial immunity. Curr. Opin. Immunol. 2003, 15 (Copyright (C) 2012 American Chemical Society (ACS). All Rights Reserved.), 112-119.

143. Lenzini, L.; Rottoli, P.; Rottoli, L., The spectrum of human tuberculosis. Clin Exp Immunol 1977, 27 (Copyright (C) 2012 U.S. National Library of Medicine.), 2307.

144. Matsunaga, I.; Sugita, M., Lipid-specific immune responses against tuberculosis: from basic science to medical applications. Curr. Immunol. Rev. 2007, 3 (Copyright (C) 2012 American Chemical Society (ACS). All Rights Reserved.), 145-150.

145. Kinjo, Y.; Wu, D.; Kim, G.; Xing, G.-W.; Poles, M. A.; Ho, D. D.; Tsuji, M.; Kawahara, K.; Wong, C.-H.; Kronenberg, M., Recognition of bacterial glycosphingolipids by natural killer T cells. Nature (London, U. K.) 2005, 434 (Copyright (C) 2012 American Chemical Society (ACS). All Rights Reserved.), 520-525.

146. Moody, D. B.; Young, D. C.; Cheng, T.-Y.; Rosat, J.-P.; Roura-mir, C.; O'Connor, P. B.; Zajonc, D. M.; Walz, A.; Miller, M. J.; Levery, S. B.; Wilson, I. A.; Costello, C. E.; Brenner, M. B., T Cell Activation by Lipopeptide Antigens. Science (Washington, DC, U. S.) 2004, 303 (Copyright (C) 2012 American Chemical Society (ACS). All Rights Reserved.), 527-531.

147. Kaufmann, S. H. E., Immunity to intracellular bacteria. Annu. Rev. Immunol. 1993, 11 (Copyright (C) 2012 American Chemical Society (ACS). All Rights Reserved.), 129-63.

148. Rook, G. A. W.; Attiyah, R. A.; Foley, N., The role of cytokines in the immunopathology of tuberculosis, and the regulation of agalactosyl IgG. Lymphokine Res. 1989, 8 (Copyright (C) 2012 American Chemical Society (ACS). All Rights Reserved.), 323-8. 
149. Coates, A. R. M.; Halls, G.; Hu, Y., Novel classes of antibiotics or more of the same? Br. J. Pharmacol. 2011, 163 (Copyright (C) 2012 American Chemical Society (ACS). All Rights Reserved.), 184-194.

150. Montgomery, A. B., The antibiotic pipeline. N. Engl. J. Med. 2004, 351 (Copyright (C) 2012 American Chemical Society (ACS). All Rights Reserved.), 2020.

151. Holcapek, M.; Kolarova, L.; Nobilis, M., High-performance liquid chromatography-tandem mass spectrometry in the identification and determination of phase I and phase II drug metabolites. Anal. Bioanal. Chem. 2008, 391 (Copyright (C) 2012 American Chemical Society (ACS). All Rights Reserved.), 59-78.

152. Xu, R. N.; Fan, L.; Rieser, M. J.; El-Shourbagy, T. A., Recent advances in highthroughput quantitative bioanalysis by LC-MS/MS. J. Pharm. Biomed. Anal. 2007, 44 (Copyright (C) 2012 American Chemical Society (ACS). All Rights Reserved.), 342-355.

153. Williams, R. T., Biochemical toxicology. Sci. J. 1966, 2 (Copyright (C) 2012 American Chemical Society (ACS). All Rights Reserved.), 57-61.

154. Schneider, E.; Clark, D. S., Cytochrome P450 (CYP) enzymes and the development of CYP biosensors. Biosens. Bioelectron. 2013, 39 (Copyright (C) 2012 American Chemical Society (ACS). All Rights Reserved.), 1-13.

155. Mikami, T.; Aoki, M.; Kimura, T., The application of mass spectrometry to proteomics and metabolomics in biomarker discovery and drug development. Curr. Mol. Pharmacol. 2012, 5 (Copyright (C) 2012 American Chemical Society (ACS). All Rights Reserved.), 301-316.

156. Lee, M. S.; Yost, R. A., Rapid identification of drug metabolites with tandem mass spectrometry. Biomed Environ Mass Spectrom 1988, 15 (Copyright (C) 2012 U.S. National Library of Medicine.), 193-204.

157. Chang, J., Rule for the variation of nitrogen atoms, electrons, masses and cleavage bonds spectrometric fragmentation. Daxue Huaxue 1988, 3 (Copyright (C) 2012 American Chemical Society (ACS). All Rights Reserved.), 18-21.

158. Brewer, E.; Henion, J., Atmospheric pressure ionization (API) LC/tandem MS techniques for drug disposition studies. J. Pharm. Sci. 1998, 87 (Copyright (C) 2012 American Chemical Society (ACS). All Rights Reserved.), 395-402. 
159. Huang, Y.; Herbold, R.; Stratton, T.; Sharma, S., Full scan data acquisition for rapid quantitative and qualitative analysis using the exactive benchtop LC-MS high resolution mass spectrometer. Spectra Anal. 2012, 41 (Copyright (C) 2012 American Chemical Society (ACS). All Rights Reserved.), 72-75.

160. Madhura, D., Preliminary Rat Liver S9 Microsomal Metabolic Stabilities of Novel Spectinomycin Derivative Analogs. In Pharmaceutical Sciences University of Tennessee Health Science Center: 2012; p 12.

161. Madhura, D., Trivedi, A, Rathi, C, Liu, J, Lee, RE, Meibohm, B. Pharmacokinetics of Novel Spectinamides. AAPS Journal 2012; 14(S2). , 2012.

162. Wind, M.; Spickermann, J.; Schleimer, M.; Donzelli, M.; Gebhardt, K.; SturmHaurany, R.; Klauer, D.; Fullhardt, P.; Schmitt-Hoffmann, A., Investigation of low-abundant in vitro metabolites of stable isotope-labelled BAL4815 by accurate mass capillary-LC-ESI-qTof-MS and MS/MS. J. Mass Spectrom. 2006, 41 (Copyright (C) 2012 American Chemical Society (ACS). All Rights Reserved.), 903-910.

163. Luo, Y.; Liu, C.; Qu, Y.; Fang, N., Towards single-cell analysis for pharmacokinetics. Bioanalysis 2012, 4 (Copyright (C) 2012 American Chemical Society (ACS). All Rights Reserved.), 453-463.

164. Shaw, K. J.; Barbachyn, M. R., The oxazolidinones: past, present, and future. Ann. N. Y. Acad. Sci. 2011, 1241 (Copyright (C) 2012 American Chemical Society (ACS). All Rights Reserved.), 48-70.

165. Hurdle, J. G.; Lee, R. B.; Budha, N. R.; Carson, E. I.; Qi, J.; Scherman, M. S.; Cho, S. H.; McNeil, M. R.; Lenaerts, A. J.; Franzblau, S. G.; Meibohm, B.; Lee, R. E., A microbiological assessment of novel nitrofuranylamides as antituberculosis agents. J. Antimicrob. Chemother. 2008, 62 (Copyright (C) 2012 American Chemical Society (ACS). All Rights Reserved.), 1037-1045.

166. Tangallapally, R. P.; Yendapally, R.; Lee, R. E.; Hevener, K.; Jones, V. C.; Lenaerts, A. J. M.; McNeil, M. R.; Wang, Y.; Franzblau, S.; Lee, R. E., Synthesis and Evaluation of Nitrofuranylamides as Novel Antituberculosis Agents. J. Med. Chem. 2004, 47 (Copyright (C) 2012 American Chemical Society (ACS). All Rights Reserved.), 5276-5283.

167. Rakesh; Bruhn, D.; Madhura, D. B.; Maddox, M.; Lee, R. B.; Trivedi, A.; Yang, L.; Scherman, M. S.; Gilliland, J. C.; Gruppo, V.; McNeil, M. R.; Lenaerts, A. J.; Meibohm, B.; Lee, R. E., Antitubercular nitrofuran isoxazolines with improved pharmacokinetic properties. Bioorg. Med. Chem. 2012, 20 (Copyright (C) 2012 American Chemical Society (ACS). All Rights Reserved.), 6063-6072. 
168. Adam, G. C.; Sorensen, E. J.; Cravatt, B. F., Chemical strategies for functional proteomics. Mol. Cell. Proteomics 2002, 1 (Copyright (C) 2012 American Chemical Society (ACS). All Rights Reserved.), 781-790.

169. Cravatt, B. F. In Activity-based protein profiling: Chemical approaches for functional proteomics, American Chemical Society: 2002; pp BIOL-011.

170. Speers, A. E.; Cravatt, B. F., Profiling enzyme activities in vivo using click chemistry methods. Chem Biol 2004, 11 (Copyright (C) 2012 U.S. National Library of Medicine.), 535-46.

171. Jessani, N.; Cravatt, B. F., The development and application of methods for activity-based protein profiling. Curr. Opin. Chem. Biol. 2004, 8 (Copyright (C) 2012 American Chemical Society (ACS). All Rights Reserved.), 54-59.

172. Jessani, N.; Cravatt, B. F. In Chemical strategies for activity-based proteomics, Wiley-VCH Verlag GmbH \& Co. KGaA: 2007; pp 403-426.

173. Madsen, M. A.; Deryugina, E. I.; Niessen, S.; Cravatt, B. F.; Quigley, J. P., Activity-based Protein Profiling Implicates Urokinase Activation as a Key Step in Human Fibrosarcoma Intravasation. J. Biol. Chem. 2006, 281 (Copyright (C) 2012 American Chemical Society (ACS). All Rights Reserved.), 15997-16005.

174. Chiang, K. P.; Niessen, S.; Saghatelian, A.; Cravatt, B. F., An Enzyme that Regulates Ether Lipid Signaling Pathways in Cancer Annotated by Multidimensional Profiling. Chem. Biol. (Cambridge, MA, U. S.) 2006, 13 (Copyright (C) 2012 American Chemical Society (ACS). All Rights Reserved.), 1041-1050.

175. Diacon, A. H.; Dawson, R.; Hanekom, M.; Narunsky, K.; Venter, A.; Hittel, N.; Geiter, L. J.; Wells, C. D.; Paccaly, A. J.; Donald, P. R., Early bactericidal activity of delamanid (OPC-67683) in smear-positive pulmonary tuberculosis patients. Int J Tuberc Lung Dis 2011, 15 (Copyright (C) 2012 U.S. National Library of Medicine.), 949-54.

176. Singh, R.; Manjunatha, U.; Boshoff, H. I. M.; Ha, Y. H.; Niyomrattanakit, P.; Ledwidge, R.; Dowd, C. S.; Lee, I. Y.; Kim, P.; Zhang, L.; Kang, S.; Keller, T. H.; Jiricek, J.; Barry, C. E., III, PA-824 Kills Nonreplicating Mycobacterium tuberculosis by Intracellular NO Release. Science (Washington, DC, U. S.) 2008, 322 (Copyright (C) 2012 American Chemical Society (ACS). All Rights Reserved.), 1392-1395.

177. Kolb, H. C.; Finn, M. G.; Sharpless, K. B., Click chemistry: diverse chemical function from a few good reactions. Angew. Chem., Int. Ed. 2001, 40 (Copyright (C) 2012 American Chemical Society (ACS). All Rights Reserved.), 2004-2021. 
178. Hein, J. E.; Tripp, J. C.; Krasnova, L. B.; Sharpless, K. B.; Fokin, V. V., Copper(I)-catalyzed cycloaddition of organic azides and 1-iodoalkynes. Angew. Chem., Int. Ed. 2009, 48 (Copyright (C) 2012 American Chemical Society (ACS). All Rights Reserved.), 8018-8021, S8018/1-S8018/64.

179. Sanchez, G. M.; De, L. T. M. A.; Morales, G.; Peliez, B.; Tolon, M. J.; Domingo, S.; Candel, F. J.; Andrade, R.; Arribi, A.; Garcia, N.; Martinez, S. F.; Fereres, J.; Picazo, J., Clinical outbreak of linezolid-resistant Staphylococcus aureus in an intensive care unit. JAMA, J. Am. Med. Assoc. 2010, 303 (Copyright (C) 2012 American Chemical Society (ACS). All Rights Reserved.), 2260-2264.

180. Sinz, M. W.; Podoll, T. In The mass spectrometer in drug metabolism, Marcel Dekker, Inc.: 2002; pp 271-335.

181. Baillie, T. A., Advances in the application of mass spectrometry to studies of drug metabolism, pharmacokinetics and toxicology. Int. J. Mass Spectrom. Ion Processes 1992, 118-119 (Copyright (C) 2012 American Chemical Society (ACS). All Rights Reserved.), 289-314. 


\section{APPENDIX A. MASS DIFFERENTIAL OF COMMON METABOLITES}

The following tables serve to illustrate the nominal mass change(s) indicative of common metabolic transformations both via $\mathrm{MS}^{180}$ (Table 3) and $\mathrm{MS} / \mathrm{MS}^{181}$ (Table 4) scanning modes.

Table 3. Common Metabolic Reactions and Their Associated Nominal Mass Differences

\begin{tabular}{lc}
\hline Conjugate & $\mathbf{m} / \mathbf{z}$ \\
\hline Acetylation & 42 \\
Carbon Hydroxylation & 16 \\
Demethylation & -14 \\
Epoxidation & 16 \\
Glucuronidation & 176 \\
Glutamine Conjugation & 145 \\
Glycine Conjugation & 57 \\
Methylation & 14 \\
N-Hydroxylation & 16 \\
N-Oxidation & 16 \\
Oxidative Desulfuration & -32 \\
Phosphorus Oxidation & $16 / 17$ \\
Sulfation & 80 \\
Taurine Conjugation & 107 \\
\hline
\end{tabular}


Table 4. Characteristic Fragments of Drug Conjugation via MS/MS Scanning

\begin{tabular}{lllc}
\hline Conjugate & Ionization Mode & MS/MS Scan & Mass \\
\hline Aliphatic Sulfates & Negative & Precursor & 97 \\
Aliphatic-GSH & Positive & Neutral Loss & 129 \\
Aryl-GSH & Positive & Neutral Loss & 275 \\
Carnitine Butyl Esters & Positive & Precursor & 103 \\
Coenzyme A Thioesters & & & \\
$(-)$ & Negative & Precursor & 329,358 \\
Coenzyme A Thioesters & & & \\
$(+)$ & Positive & Precursor & 428 \\
Glucuronides & Both & Neutral Loss & 176 \\
N-Acetylcysteines & Negative & Neutral Loss & 129 \\
Phenolic sulfates & Positive & Neutral Loss & 89 \\
Phosphates & Negative & Precursor & 63,79 \\
Sulfinates & Negative & Neutral Loss & 64 \\
Sulfonates & Negative & Precursor & 81 \\
Taurines & Positive & Precursor & 126 \\
Taurines & Negative & Precursor & 339,358 \\
\hline
\end{tabular}




\section{APPENDIX B. STRUCTURES OF FAME GC/MS PEAKS \#2 - \#11}

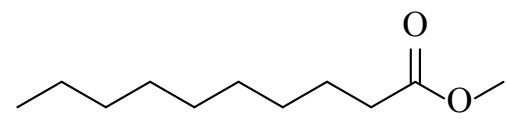

Chemical Formula: $\mathrm{C}_{11} \mathrm{H}_{22} \mathrm{O}_{2}$

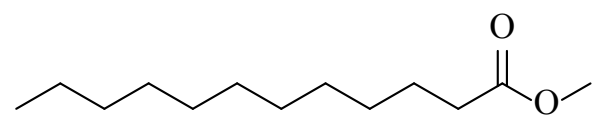

Chemical Formula: $\mathrm{C}_{13} \mathrm{H}_{26} \mathrm{O}_{2}$<smiles>CCCCCCCCC(O)C(=O)OC</smiles>

Chemical Formula: $\mathrm{C}_{11} \mathrm{H}_{22} \mathrm{O}_{3}$

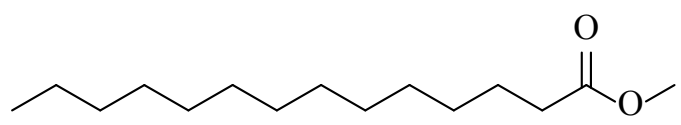

Chemical Formula: $\mathrm{C}_{15} \mathrm{H}_{30} \mathrm{O}_{2}$<smiles>CCCCCCCCCCCCCCCC(=O)OC</smiles>

Chemical Formula: $\mathrm{C}_{17} \mathrm{H}_{34} \mathrm{O}_{2}$

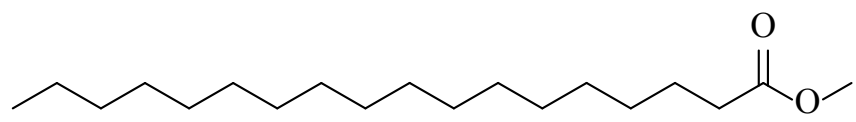

Chemical Formula: $\mathrm{C}_{19} \mathrm{H}_{38} \mathrm{O}_{2}$
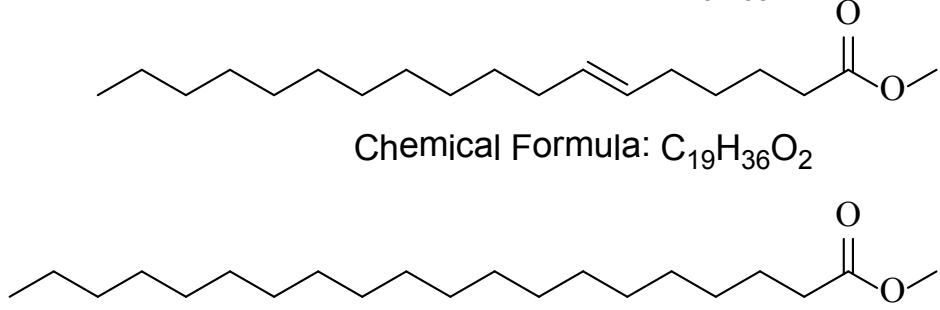

Chemical Formula: $\mathrm{C}_{21} \mathrm{H}_{42} \mathrm{O}_{2}$

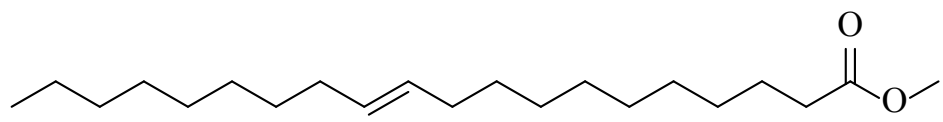

Chemical Formula: $\mathrm{C}_{21} \mathrm{H}_{40} \mathrm{O}_{2}$

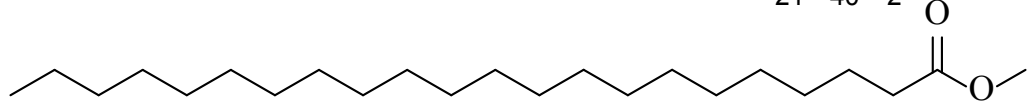

Chemical Formula: $\mathrm{C}_{23} \mathrm{H}_{46} \mathrm{O}_{2}$

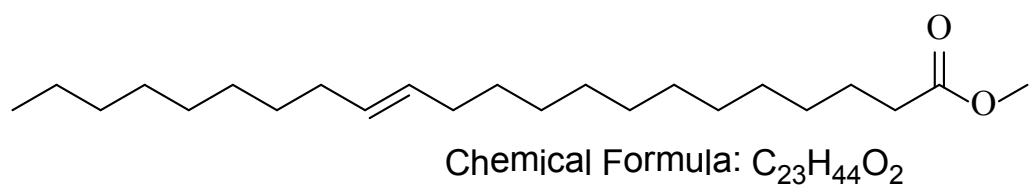


APPENDIX C. SUPPORTING PK INFORMATION

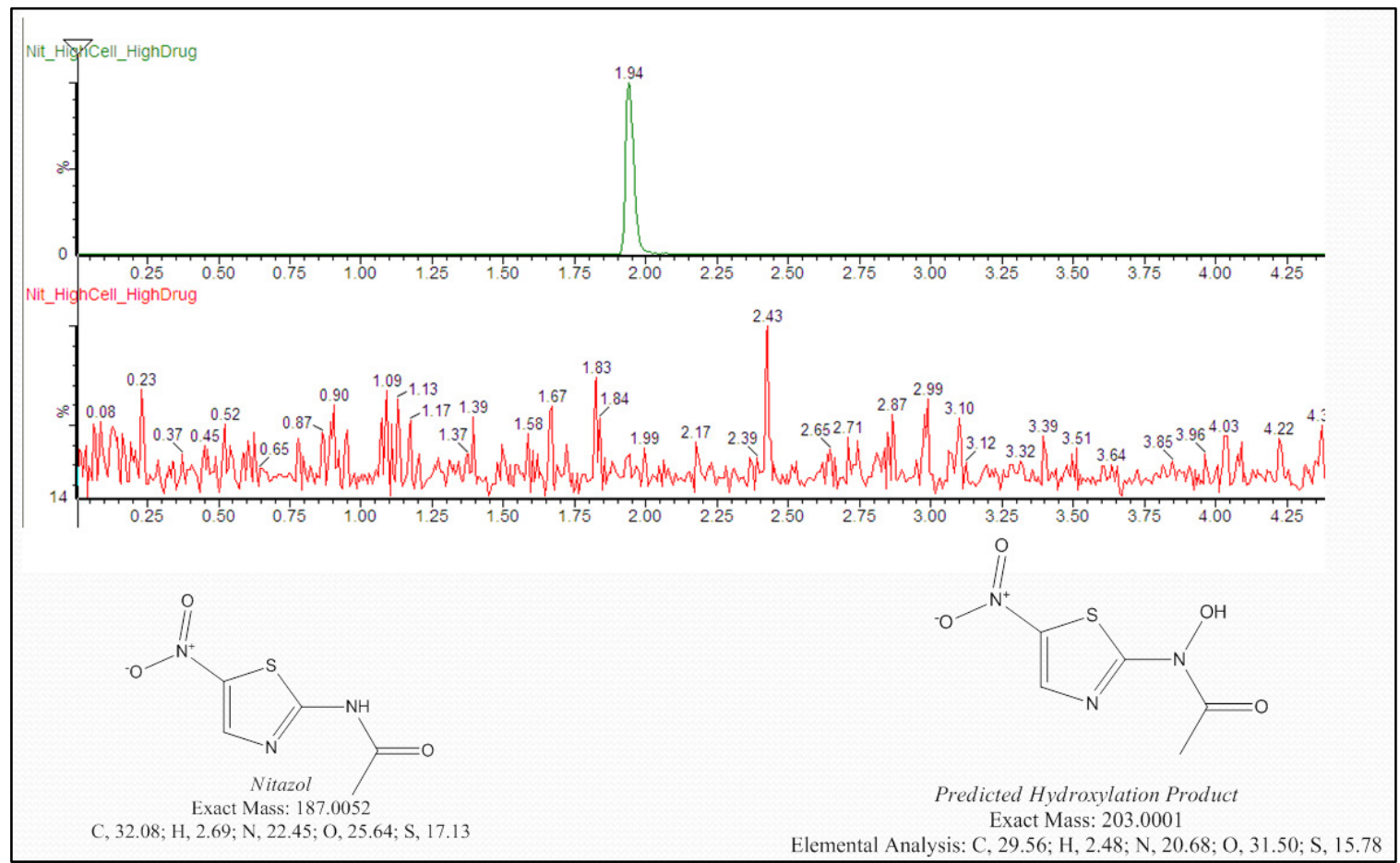

Figure 69. HRMS Identification of Nitazol Metabolite 


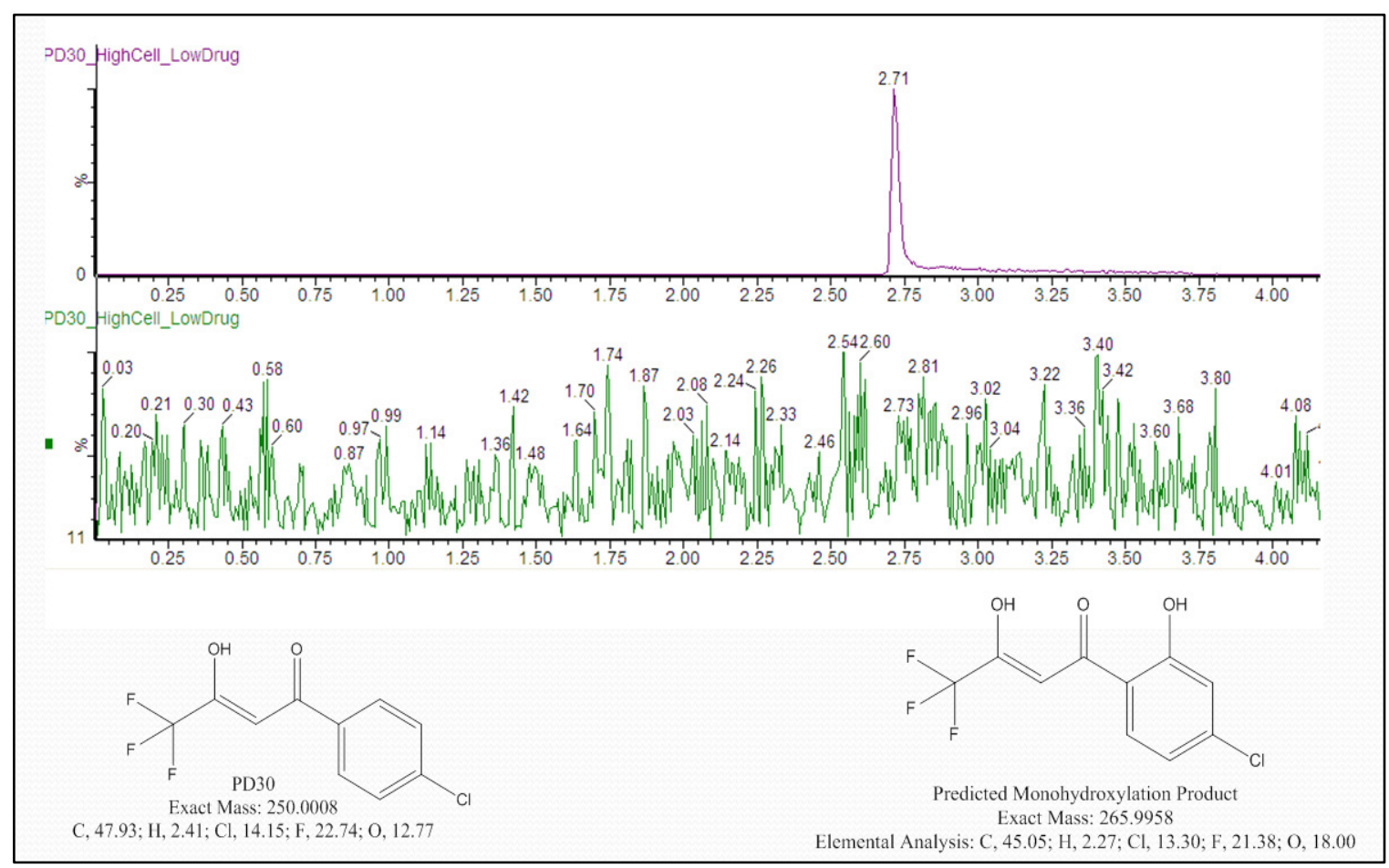

Figure 70. HRMS Idenification of PD30 Metabolite 


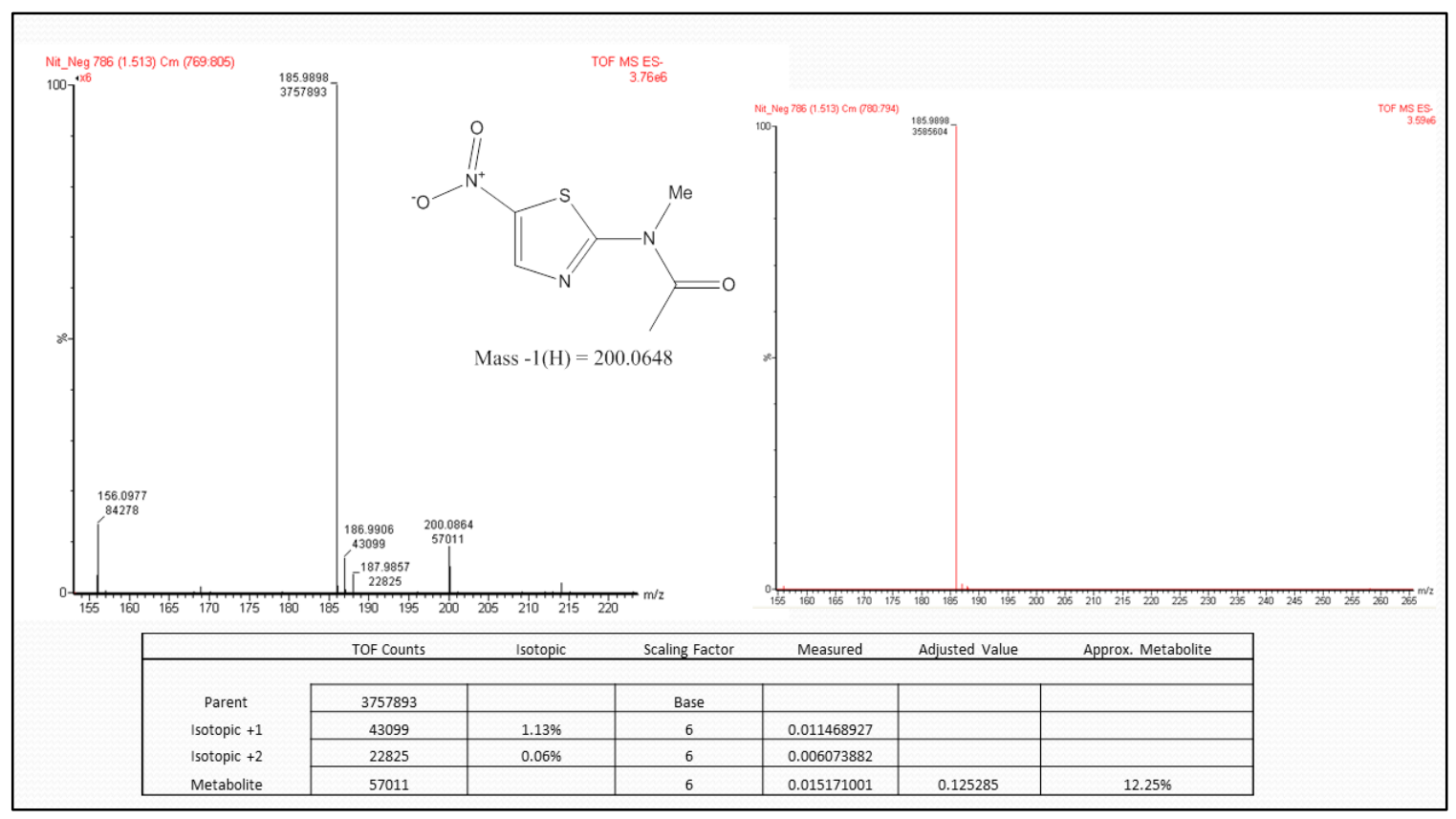

Figure 71 Quantification of Nitazol Metabolite 


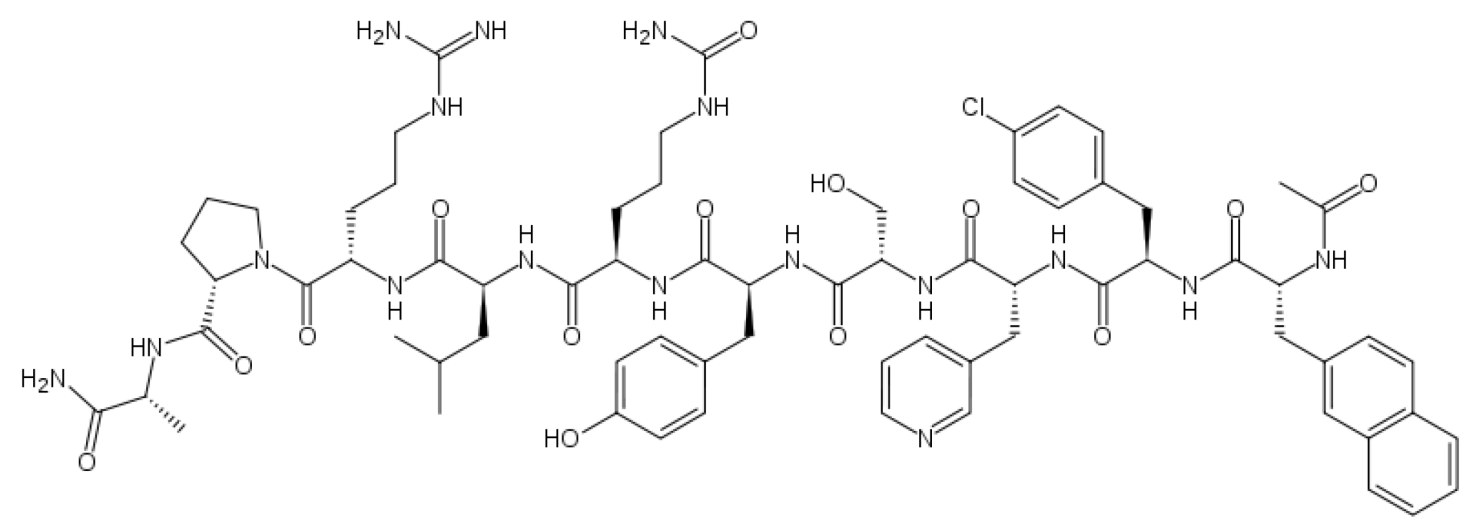

Figure 72. Structure of Cetrorelix 


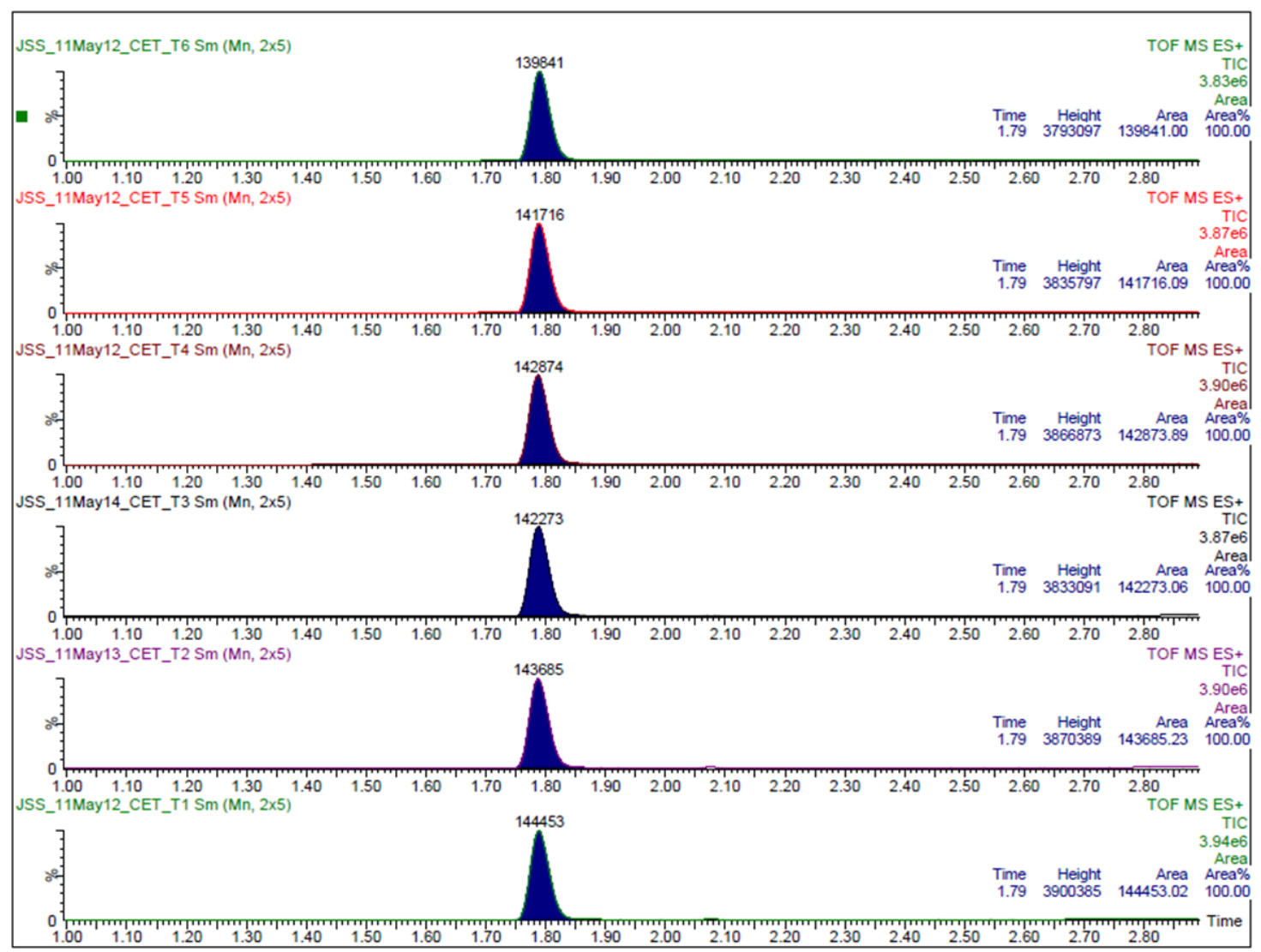

Figure 73. HRMS Stability Study of Cetrorelix 
Table 5. Calculated Cetrorelix Stability

\begin{tabular}{lcc}
\hline Sample Lot & Sample & Peak Area \\
\hline 5-May-11 & T1 & 139899 \\
& T2 & 136344 \\
& T3 & 141620 \\
6-May-11 & T4 & 142874 \\
& T5 & 141716 \\
& T6 & 139841 \\
Mean (T) & & 140382 \\
After 7 Days & O1 & 71464 \\
& O2 & 71580 \\
& O3 & 69584 \\
Mean (O) & & 70876 \\
Blank & & 59 \\
& B1 & 67 \\
Mean Blank & B2 & 48 \\
Mean Lifespan (hrs) & B3 & 58 \\
Mean Degradation (\% avg) & & 192 \\
Mean Lifespan (Calculated) & & 0.504673696 \\
\hline
\end{tabular}




\section{APPENDIX A. MASS SPECTROMETER PARAMETERS}

TQD MS1 Parameters

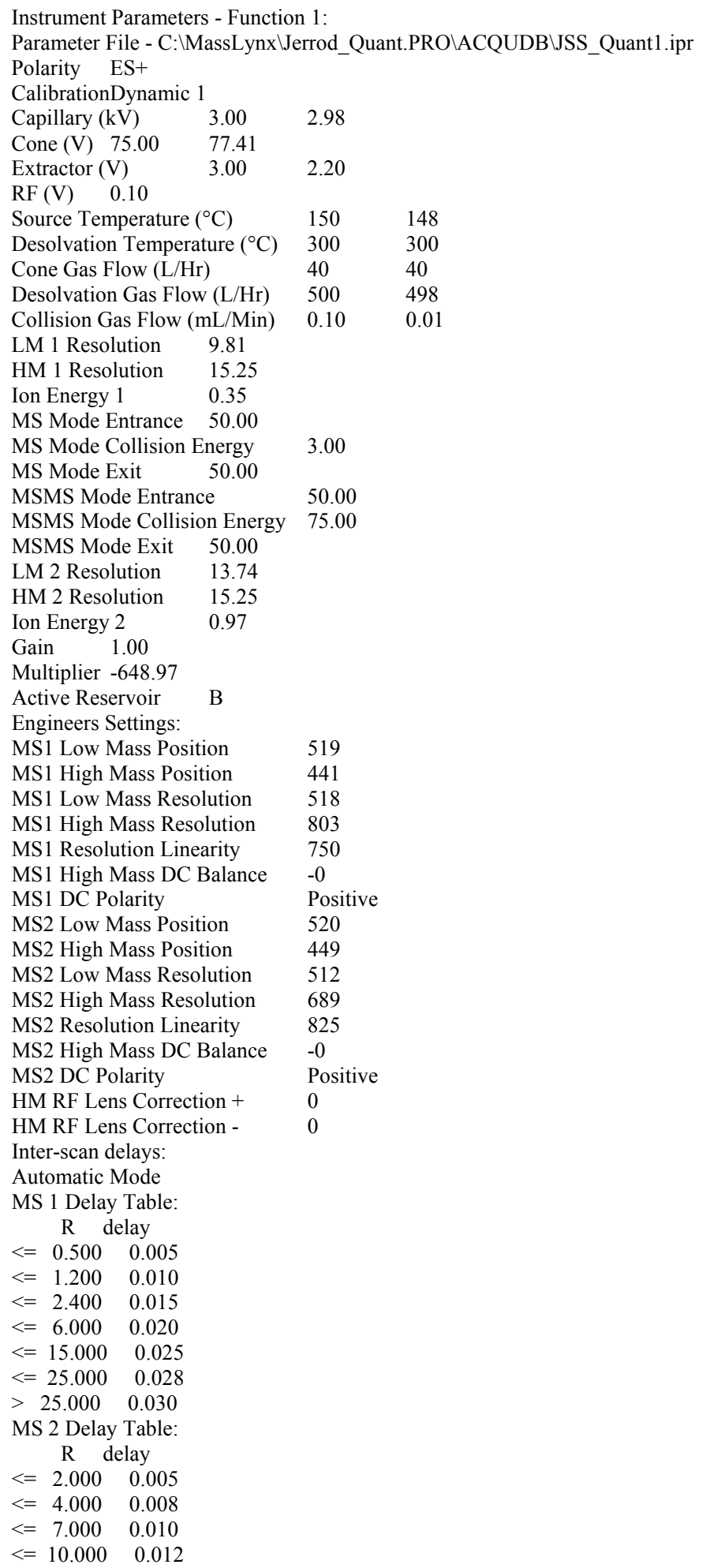




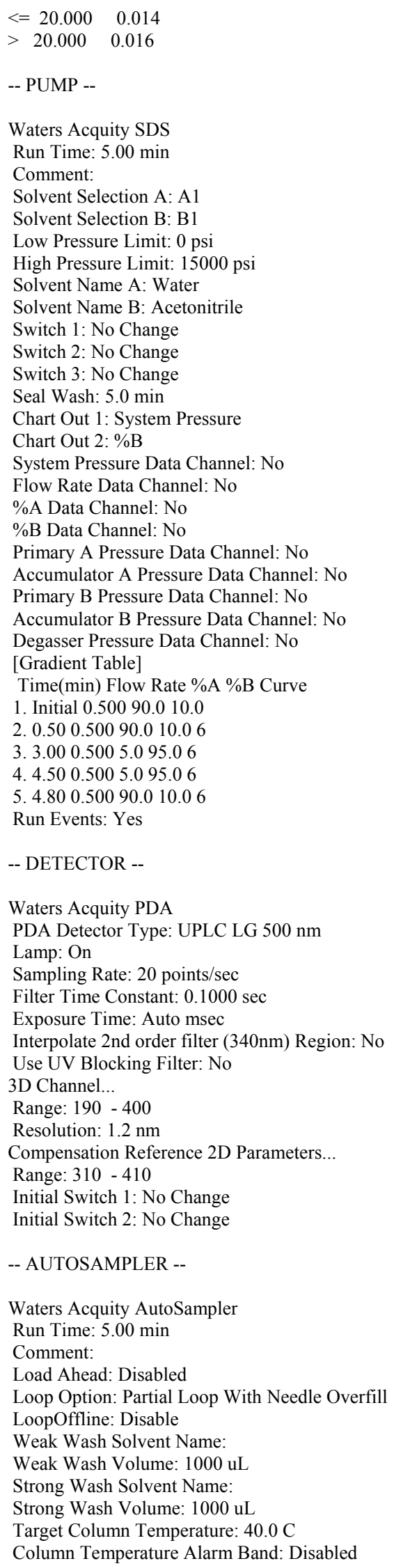


Target Sample Temperature: Off C

Sample Temperature Alarm Band: Disabled

Full Loop Overfill Factor: Automatic

Syringe Draw Rate: Automatic

Needle Placement: 8.0

Pre-Aspirate Air Gap: Automatic

Post-Aspirate Air Gap: Automatic

Column Temperature Data Channel: No

Ambient Temperature Data Channel: No

Sample Temperature Data Channel: No

Sample Organizer Temperature Data Channel: No

Sample Pressure Data Channel: No

Switch 1: No Change

Switch 2: No Change

Switch 3: No Change

Switch 4: No Change

Chart Out: Sample Pressure

Sample Temp Alarm: Disabled

Column Temp Alarm: Disabled

Run Events: Yes

Needle Overfill Flush: Automatic

Sample Loop Size: 10.0

Sample Run Injection Parameter

Injection Volume (ul) - 7.50

Cycle time (secs): $\quad-1.000$

Scan duration (secs): $\quad 0.15$

Interscan delay (secs): $\quad-1.00$

Retention window (mins): 0.000 to 5.000

Ionization mode: $\quad \mathrm{ES}+$

Data type: Accurate Mass

Function type: Scan

Mass range: $\quad 300$ to 900

Function 2

Scans in function: 6001

Function type: Diode Array

Wavelength range ( $\mathrm{nm}$ ) : 190 to 400

\section{TQD MS/MS Parameters}

Instrument Parameters - Function 1:

Parameter File - C:IMassLynx \Jerrod_Quant.PRO \ACQUDB $\backslash J S S \_Q u a n t 1 . i p r$

Polarity ES+

CalibrationDynamic 2

$\begin{array}{lll}\text { Capillary }(\mathrm{kV}) & 3.00 & 2.98\end{array}$

Cone (V) $75.00 \quad 76.43$

$\begin{array}{lll}\text { Extractor }(\mathrm{V}) & 3.00 & 2.69\end{array}$

$\mathrm{RF}(\mathrm{V}) \quad 0.10$

Source Temperature $\left({ }^{\circ} \mathrm{C}\right) \quad 150 \quad 148$

Desolvation Temperature $\left({ }^{\circ} \mathrm{C}\right) \quad 300 \quad 300$

Cone Gas Flow (L/Hr) $\quad 40 \quad 39$

Desolvation Gas Flow (L/Hr) $\quad 500 \quad 498$

Collision Gas Flow (mL/Min) $\quad 0.10 \quad 0.10$

LM 1 Resolution 9.81

HM 1 Resolution $\quad 15.25$

Ion Energy $1 \quad 0.35$

MS Mode Entrance $\quad 50.00$

MS Mode Collision Energy

MS Mode Exit $\quad 50.00$

MSMS Mode Entrance

MSMS Mode Exit $\quad 50.00$

LM 2 Resolution $\quad 13.74$

HM 2 Resolution $\quad 15.25$

Ion Energy $2 \quad 0.97$

Gain $\quad 1.00$

Multiplier -648.97

Active Reservoir

Engineers Settings:

MS1 Low Mass Position 
$\begin{array}{ll}\text { MS1 High Mass Position } & 441 \\ \text { MS1 Low Mass Resolution } & 518 \\ \text { MS1 High Mass Resolution } & 803 \\ \text { MS1 Resolution Linearity } & 750 \\ \text { MS1 High Mass DC Balance } & -0 \\ \text { MS1 DC Polarity } & \text { Positive } \\ \text { MS2 Low Mass Position } & 520 \\ \text { MS2 High Mass Position } & 449 \\ \text { MS2 Low Mass Resolution } & 512 \\ \text { MS2 High Mass Resolution } & 689 \\ \text { MS2 Resolution Linearity } & 825 \\ \text { MS2 High Mass DC Balance } & -0 \\ \text { MS2 DC Polarity } & \text { Positive } \\ \text { HM RF Lens Correction + } & 0 \\ \text { HM RF Lens Correction - } & 0\end{array}$

Inter-scan delays:

Automatic Mode

MS 1 Delay Table:

$\mathrm{R}$ delay

$<=0.500 \quad 0.005$

$<=1.200 \quad 0.010$

$<=2.400 \quad 0.015$

$<=6.000 \quad 0.020$

$<=15.000 \quad 0.025$

$<=25.000 \quad 0.028$

$>25.000 \quad 0.030$

MS 2 Delay Table:

$\mathrm{R}$ delay

$<=2.000 \quad 0.005$

$<=4.000 \quad 0.008$

$<=7.000 \quad 0.010$

$<=10.000 \quad 0.012$

$<=20.000 \quad 0.014$

$>20.000 \quad 0.016$

-- PUMP --

Waters Acquity SDS

Run Time: $5.00 \mathrm{~min}$

Comment:

Solvent Selection A: A1

Solvent Selection B: B1

Low Pressure Limit: 0 psi

High Pressure Limit: 15000 psi

Solvent Name A: Water

Solvent Name B: Acetonitrile

Switch 1: No Change

Switch 2: No Change

Switch 3: No Change

Seal Wash: $5.0 \mathrm{~min}$

Chart Out 1: System Pressure

Chart Out 2: \%B

System Pressure Data Channel: No

Flow Rate Data Channel: No

\%A Data Channel: No

\%B Data Channel: No

Primary A Pressure Data Channel: No

Accumulator A Pressure Data Channel: No

Primary B Pressure Data Channel: No

Accumulator B Pressure Data Channel: No

Degasser Pressure Data Channel: No

[Gradient Table]

Time(min) Flow Rate \%A \%B Curve

1. Initial 0.50090 .010 .0

2. 0.500 .50090 .010 .06

3. 3.000 .5005 .095 .06

4. 4.500 .5005 .095 .06

5. 4.800 .50090 .010 .06

Run Events: Yes 
Waters Acquity PDA

PDA Detector Type: UPLC LG $500 \mathrm{~nm}$

Lamp: On

Sampling Rate: 20 points/sec

Filter Time Constant: $0.1000 \mathrm{sec}$

Exposure Time: Auto msec

Interpolate 2nd order filter (340nm) Region: No

Use UV Blocking Filter: No

3D Channel...

Range: 190 - 400

Resolution: $1.2 \mathrm{~nm}$

Compensation Reference 2D Parameters...

Range: 310 - 410

Initial Switch 1: No Change

Initial Switch 2: No Change

\section{-- AUTOSAMPLER --}

Waters Acquity AutoSampler

Run Time: $5.00 \mathrm{~min}$

Comment:

Load Ahead: Disabled

Loop Option: Partial Loop With Needle Overfill

LoopOffline: Disable

Weak Wash Solvent Name:

Weak Wash Volume: $1000 \mathrm{uL}$

Strong Wash Solvent Name:

Strong Wash Volume: $1000 \mathrm{uL}$

Target Column Temperature: $40.0 \mathrm{C}$

Column Temperature Alarm Band: Disabled

Target Sample Temperature: Off C

Sample Temperature Alarm Band: Disabled

Full Loop Overfill Factor: Automatic

Syringe Draw Rate: Automatic

Needle Placement: 8.0

Pre-Aspirate Air Gap: Automatic

Post-Aspirate Air Gap: Automatic

Column Temperature Data Channel: No

Ambient Temperature Data Channel: No

Sample Temperature Data Channel: No

Sample Organizer Temperature Data Channel: No

Sample Pressure Data Channel: No

Switch 1: No Change

Switch 2: No Change

Switch 3: No Change

Switch 4: No Change

Chart Out: Sample Pressure

Sample Temp Alarm: Disabled

Column Temp Alarm: Disabled

Run Events: Yes

Needle Overfill Flush: Automatic

Sample Loop Size: 10.0

Sample Run Injection Parameter

Injection Volume (ul) - 7.50

Function 1

Scans in function: $\quad 1659$

Cycle time (secs): $\quad-1.000$

Scan duration (secs): $\quad 0.15$

Interscan delay (secs): $\quad-1.00$

Retention window (mins): 0.000 to 5.000

Ionization mode: $\quad \mathrm{ES}+$

Data type: Accurate Mass

Function type: Daughters of 765.00

Mass range: $\quad 300$ to 600

Collision Energy: $\quad 50.0$

Function 2

Scans in function: 6001

Function type: Diode Array 


\section{HRMS Positive Mode Parameters}

Function Parameters - Function 1 - TOF MS FUNCTION

Scan Time (sec)

Interscan Time (sec) $\quad 0.014$

Start Mass $\quad 200.0$

End Mass $\quad 900.0$

Start Time (mins) $\quad 0.00$

End Time (mins)

Data Format

ADC Sample Frequency $(\mathrm{GHz})$

ADC Pusher Frequency $(\mu \mathrm{s})$

ADC Pusher Width ( $\mu$ s)

Use Tune Page Cone Voltage

CV Ramp Start Voltage

CV Ramp End Voltage

$0.00 \quad 5.00$

Using Auto Transfer Collision Energy (eV) 6.000000

Sensitivity

Dynamic Range

Save Collapsed Retention Time Data

Use Rule File Filtering

Calibration

$\begin{array}{ll}6.0 & \text { Continuum } \\ 60.0 & \\ & 1.50 \\ \text { NO } & \\ & 15.0 \\ & 50.0 \\ \text { Normal } & \\ \text { No } & \text { Normal } \\ & \text { No }\end{array}$

-- PUMP --

Waters Acquity SDS

Run Time: $5.00 \mathrm{~min}$

Comment:

Solvent Selection A: A1

Solvent Selection B: B1

Low Pressure Limit: 0 psi

High Pressure Limit: 15000 psi

Solvent Name A: Water

Solvent Name B: Acetonitrile

Switch 1: No Change

Switch 2: No Change

Switch 3: No Change

Seal Wash: $5.0 \mathrm{~min}$

Chart Out 1: System Pressure

Chart Out 2: \%B

System Pressure Data Channel: No

Flow Rate Data Channel: No

\%A Data Channel: No

\%B Data Channel: No

Primary A Pressure Data Channel: No

Accumulator A Pressure Data Channel: No

Primary B Pressure Data Channel: No

Accumulator B Pressure Data Channel: No

Degasser Pressure Data Channel: No

[Gradient Table]

Time(min) Flow Rate \%A \%B Curve

1. Initial 0.40098 .02 .0

2. 0.500 .40098 .02 .06

3. 3.750 .4005 .095 .06

4. 4.500 .4005 .095 .06

5. 4.600 .40098 .02 .06

6. 5.000 .40098 .02 .06

Run Events: Yes

-- DETECTOR --

Waters Acquity TUV

Run Time: $5.00 \mathrm{~min}$

Wavelength Mode: Dual Wavelength

Lamp On: On

Channel A...

Comment: 
Wavelength: $254 \mathrm{~nm}$

Sampling Rate: 2 points/sec

Data Mode: Absorbance A

Time Constant: $1.0000 \mathrm{sec}$

Auto Zero On Wavelength Change: Maintain Baseline

Auto Zero On Inject Start: Yes

Channel B...

Comment:

Wavelength: $220 \mathrm{~nm}$

Sampling Rate: 2 points/sec

Data Mode: Absorbance B

Time Constant: $1.0000 \mathrm{sec}$

Auto Zero On Wavelength Change: Maintain Baseline

Auto Zero On Inject Start: Yes

Analog 1...

Sensitivity: 2.000 AUFS

Chart Polarity: Positive (+)

Voltage Offset: $0 \mathrm{mV}$

Enable Chart Mark: Yes

Analog 2...

Sensitivity: 2.000 AUFS

Chart Polarity: Positive $(+)$

Voltage Offset: $0 \mathrm{mV}$

Enable Chart Mark: Yes

Run Events: Yes

Pulse Width: $1.0 \mathrm{sec}$

Rect Wave Period: $0.2 \mathrm{sec}$

-- AUTOSAMPLER --

Waters Acquity AutoSampler

Run Time: $5.00 \mathrm{~min}$

Comment:

Load Ahead: Disabled

Loop Option: Partial Loop

LoopOffline: Disable

Weak Wash Solvent Name:

Weak Wash Volume: $1000 \mathrm{uL}$

Strong Wash Solvent Name:

Strong Wash Volume: $500 \mathrm{uL}$

Target Column Temperature: $37.0 \mathrm{C}$

Column Temperature Alarm Band: Disabled

Target Sample Temperature: Off C

Sample Temperature Alarm Band: Disabled

Full Loop Overfill Factor: Automatic

Syringe Draw Rate: Automatic

Needle Placement: Automatic

Pre-Aspirate Air Gap: Automatic

Post-Aspirate Air Gap: Automatic

Column Temperature Data Channel: No

Ambient Temperature Data Channel: No

Sample Temperature Data Channel: No

Sample Organizer Temperature Data Channel: No

Sample Pressure Data Channel: No

Switch 1: No Change

Switch 2: No Change

Switch 3: No Change

Switch 4: No Change

Chart Out: Sample Pressure

Sample Temp Alarm: Disabled

Column Temp Alarm: Disabled

Run Events: Yes

Needle Overfill Flush: Automatic

Sample Loop Size: 10.0

Sample Run Injection Parameter

Injection Volume (ul) - 5.00

Function 1

Scans in function: 581

Cycle time (secs): $\quad 0.514$

Scan duration (secs): $\quad 0.50$ 
Interscan delay (secs): $\quad 0.01$

Retention window (mins): 0.000 to 5.000

Ionization mode:

$\mathrm{ES}+$

Data type:

Function type:

Enhanced Mass

Mass range: 200 to 900

Function 2

Scans in function: 601

Function type: Diode Array

Wavelength range $(\mathrm{nm}): 220$ to 254

\section{HRMS Negative Mode Parameters}

Function Parameters - Function 1 - TOF MS FUNCTION

Scan Time (sec)

0.500

Interscan Time $(\mathrm{sec})$

0.014

200.0

End Mass $\quad 900.0$

Start Time (mins) $\quad 0.00$

End Time (mins)

Data Format

ADC Sample Frequency $(\mathrm{GHz})$

ADC Pusher Frequency $(\mu s)$

ADC Pusher Width $(\mu \mathrm{s})$

Use Tune Page Cone Voltage

$0.00 \quad 5.00$

CV Ramp Start Voltage

6.0

Continuum

Using Auto Transfer Collision Energy (eV) 6.000000

Sensitivity

Dynamic Range

Save Collapsed Retention Time Data

Use Rule File Filtering

Calibration

60.0

NO $\quad 1.50$

15.0

50.0

Normal

No Normal

Dynamic 2

-- PUMP --

Waters Acquity SDS

Run Time: $5.00 \mathrm{~min}$

Comment:

Solvent Selection A: A1

Solvent Selection B: B1

Low Pressure Limit: 0 psi

High Pressure Limit: 15000 psi

Solvent Name A: Water

Solvent Name B: Acetonitrile

Switch 1: No Change

Switch 2: No Change

Switch 3: No Change

Seal Wash: $5.0 \mathrm{~min}$

Chart Out 1: System Pressure

Chart Out 2: \%B

System Pressure Data Channel: No

Flow Rate Data Channel: No

\%A Data Channel: No

\%B Data Channel: No

Primary A Pressure Data Channel: No

Accumulator A Pressure Data Channel: No

Primary B Pressure Data Channel: No

Accumulator B Pressure Data Channel: No

Degasser Pressure Data Channel: No

[Gradient Table]

Time(min) Flow Rate \%A \%B Curve

1. Initial 0.40098 .02 .0

2. 0.500 .40098 .02 .06

3. 3.750 .4005 .095 .06

4. 4.500 .4005 .095 .06

5. 4.600 .40098 .02 .06

6. 5.000 .40098 .02 .06

Run Events: Yes 


\section{-- DETECTOR --}

Waters Acquity TUV

Run Time: $5.00 \mathrm{~min}$

Wavelength Mode: Dual Wavelength

Lamp On: On

Channel A...

Comment:

Wavelength: $254 \mathrm{~nm}$

Sampling Rate: 2 points/sec

Data Mode: Absorbance A

Time Constant: $1.0000 \mathrm{sec}$

Auto Zero On Wavelength Change: Maintain Baseline

Auto Zero On Inject Start: Yes

Channel B...

Comment:

Wavelength: $220 \mathrm{~nm}$

Sampling Rate: 2 points/sec

Data Mode: Absorbance B

Time Constant: $1.0000 \mathrm{sec}$

Auto Zero On Wavelength Change: Maintain Baseline

Auto Zero On Inject Start: Yes

Analog 1...

Sensitivity: 2.000 AUFS

Chart Polarity: Positive (+)

Voltage Offset: $0 \mathrm{mV}$

Enable Chart Mark: Yes

Analog 2..

Sensitivity: 2.000 AUFS

Chart Polarity: Positive $(+)$

Voltage Offset: $0 \mathrm{mV}$

Enable Chart Mark: Yes

Run Events: Yes

Pulse Width: $1.0 \mathrm{sec}$

Rect Wave Period: $0.2 \mathrm{sec}$

\section{-- AUTOSAMPLER --}

Waters Acquity AutoSampler

Run Time: $5.00 \mathrm{~min}$

Comment:

Load Ahead: Disabled

Loop Option: Partial Loop

LoopOffline: Disable

Weak Wash Solvent Name:

Weak Wash Volume: $1000 \mathrm{uL}$

Strong Wash Solvent Name:

Strong Wash Volume: $500 \mathrm{uL}$

Target Column Temperature: $37.0 \mathrm{C}$

Column Temperature Alarm Band: Disabled

Target Sample Temperature: Off C

Sample Temperature Alarm Band: Disabled

Full Loop Overfill Factor: Automatic

Syringe Draw Rate: Automatic

Needle Placement: Automatic

Pre-Aspirate Air Gap: Automatic

Post-Aspirate Air Gap: Automatic

Column Temperature Data Channel: No

Ambient Temperature Data Channel: No

Sample Temperature Data Channel: No

Sample Organizer Temperature Data Channel: No

Sample Pressure Data Channel: No

Switch 1: No Change

Switch 2: No Change

Switch 3: No Change

Switch 4: No Change

Chart Out: Sample Pressure

Sample Temp Alarm: Disabled

Column Temp Alarm: Disabled 
Run Events: Yes

Needle Overfill Flush: Automatic

Sample Loop Size: 10.0

Function 1

Scans in function: 581

Cycle time (secs): $\quad 0.514$

Scan duration (secs): $\quad 0.50$

Interscan delay (secs): 0.01

Retention window (mins): 0.000 to 5.000

Ionization mode: ES-

Data type: $\quad$ Enhanced Mass

Function type: TOF MS

Mass range: $\quad 200$ to 900

Function 2

Scans in function: 601

Function type: Diode Array

Wavelength range ( $\mathrm{nm}$ ) : 220 to 254 


\section{VITA}

Jerrod Stephen Scarborough, a native of Marshall, AR, was born in 1980. He attended Arkansas State University in Jonesboro, AR, where he obtained a Bachelor's degree in Chemistry in June 2007. In July of 2007 he entered the laboratory of Dr.

Richard Lee, first as a summer student, enrolling in the University of Tennessee Health Science Center (UTHSC) under the mentorship of Dr. Lee in the Fall of 2007. Upon graduation Jerrod will join the staff of St. Jude Children's Research Hospital as a Sr. Research Technologist in the High-throughput Analytical Chemistry Facility in the Department of Chemical Therapeutics and Chemical Biology. 